\title{
Directed Evolution and Discovery of Nitroreductase Enzymes for Targeted Cell Ablation
}

\author{
Rory Fox Little
}

A thesis submitted to the Victoria University of Wellington in fulfilment for the degree of Master of Science in Biotechnology

Victoria University of Wellington 



\begin{abstract}
Nitroreductase enzymes are a superfamily of bacterial flavoproteins that can catalyze the reduction of aromatic nitro groups. The reduction of an aromatic nitro group, a highly electronegative functionality, causes a large electronic shift that can profoundly affect the activity of other substituents on the aromatic ring. For example, upon nitroreduction, initially non-toxic compounds known as prodrugs can be converted into a cytotoxic form. The ability of nitroreductases to alter the activity of compounds has lead to their development as tools for multiple biotechnological applications. Of particular note is the use of nitroreductase enzymes in combination with a nitroaromatic prodrug to study the role of specific cell populations in zebrafish (Danio rerio). Zebrafish are used as model organisms to study processes such as embryonic development and tissue regeneration. By expressing a nitroreductase enzyme in a specific tissue of a zebrafish, it is possible to selectively ablate that tissue upon administration of a prodrug. The subsequent phenotypic change induced by the ablation can provide information on the physiological role of the ablated tissue, or of the regenerative processes that can be recruited to repair the damage.
\end{abstract}

The goal of this thesis was to engineer or discover new nitroreductase enzymes that could expand the capabilities of cell ablation studies in zebrafish. In particular, this work sought to develop a system that would enable the dual, or multiplexed, ablation of two tissues independently within the same organism. Control over the ablation of two distinct tissues could be useful for studying tissue interactions during developmental or regenerative processes. For this to be achievable, two different nitroreductase enzymes, each possessing distinct and non-overlapping prodrug selectivities would be required. Previous studies in the Ackerley lab had identified NfsA from Escherichia coli (NfsA_Ec) and NfsA from Pseudomonas putida (NfsA_Pp) as nitroreductase enzymes that were slightly more selective for the prodrug tinidazole compared than metronidazole. In contrast the $\mathrm{NfsB}$ nitroreductase from Vibrio vulnificus (NfsB_Vv) was substantially more selective for metronidazole than tinidazole. To further improve the tinidazole selectivity of the NfsA enzymes, directed evolution was employed as a tool to further enhance the substrate selectivity of each enzyme. The primary outcome of this work was the evolution of an NfsA_Ec mutant that was 12 fold more selective for tinidazole over metronidazole than wild type NfsA_Ec.

In addition to engineering new enzymes for cell ablation experiments, this work also sought to discover new nitroreductase enzymes from unculturable bacteria, a previously unplumbed source. The genes and gene products of unculturable bacteria can be identified and studied by expressing fragments of their DNA in a readily culturable host such as E. coli. A variety of different screening methodologies were tested for identifying nitroreductase enzymes from eDNA inserts. The compound 4-nitroimidazole was found to be capable of detecting nitroreductase expression at the level of a single colony. While no novel nitroreductase enzymes were discovered in the scope of this work, the preliminary results are encouraging that a screening strategy centred on 4nitroimidazole in particular could successfully do so in the near future. 


\section{Acknowledgements}

First as always, thanks to my supervisor Dr. David Ackerley. Dave, I really cannot put it into words how grateful I am for everything, but will try my best. Your undergraduate lectures were so fantastic and a huge reason why I became so interested in molecular biology in the first place. Thank you for going above and beyond by providing me with the opportunity to work in your lab as a third year student. As a supervisor, your ideas, passion, and support (except for the time you put me on that very tall, very unstable stack of chairs by that swimming pool at night) have been inspiring. However my scientific career ends up, I will carry the same spirit (shadow) with me. Thank for helping me develop as a scientist by providing the conference opportunities this year. Thailand, in particular, I will never forget. Finally, thank you for making me feel so welcome to stay, yet also being so instrumental in my ability to leave.

To everyone else in the Ackerley lab, past and present, thank you for your friendship and support. Abby, Alistair, Becky, Elsie, Jasmine, Janine, Katherine, Luke, Mark, Matt, Michelle, and Richard, the lab is an incredible and fun working environment because of all of you. Around the Bays, Castlepoint, karaoke, step class, Elsie's Charades, Dominion, Maroon Platoon... too many great things to count. I will miss all of you when I leave. Thank you Michelle for your constructive criticism of my Methods section, and Luke for looking over the Introduction. Another special mention to Luke for, despite actually dressing really well, wearing matching clothes to me every weekday of the past four months (it did finally get funny again?). Thank you to my family for all of their support, I wouldn't have made it this far without your help. Varun, for teaching me his tissue culture skills. My other wonderful friends and flatmates for always being there when I needed it. Finally, thank you to Victoria University for financially supporting me this year with a Master's scholarship. 


\section{Table of Contents}

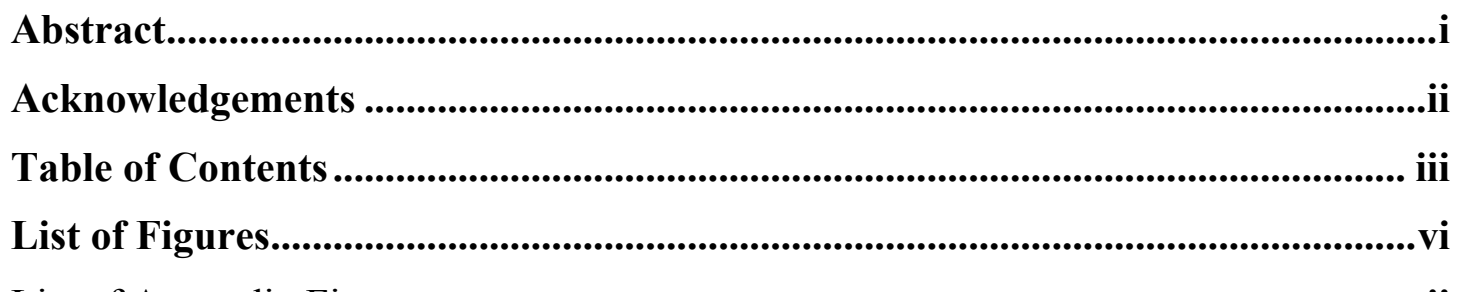

List of Appendix Figures ..........................................................................................................vii

List of Tables .............................................................................................................. viii

Abbreviations used in this Thesis................................................................................

Chapter 1 Introduction .....................................................................................................1

1.1 Nitroreductase Enzymes ................................................................................................ 1

1.2 Applications of Nitroreductase Enzymes......................................................................

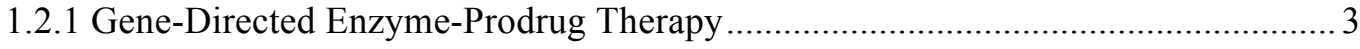

1.2.2 NfsB_Ec/CB1954 GDEPT ……………………………………………….

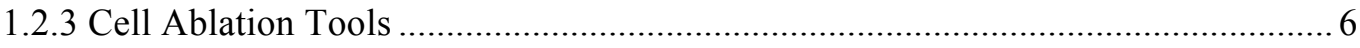

1.3 Next Generation Nitroreductase Enzymes ....................................................................11

1.3.1 Nitroreductases from Culturable Bacteria ........................................................... 11

1.3.2 Metagenomic Discovery of Nitroreductase Enzymes ............................................. 12

1.4 Engineered Nitroreductase Enzymes...............................................................................13

1.4.1 Directed Evolution ....................................................................................... 13

1.4.2 Nitroreductase Directed Evolution........................................................................... 14

1.3 Aims of this Thesis ..........................................................................................................

Chapter 2 Methods ......................................................................................................18

2.1 Oligonucleotide Primers................................................................................................ 18

2.1.1 Storage of Primers ......................................................................................... 18

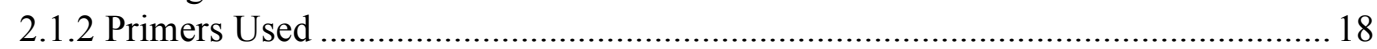

2.2 Bacteria Strains Used .................................................................................................... 20

2.3 Plasmids ......................................................................................................20

2.4 Profluorophores ...........................................................................................................23

2.5 Nitroaromatic Compounds ............................................................................................24

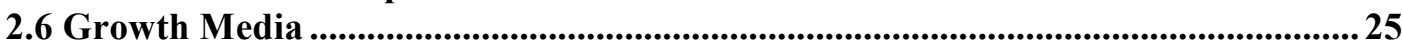

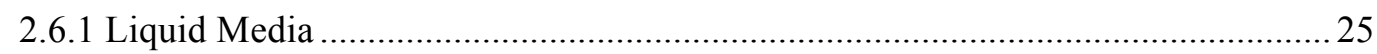

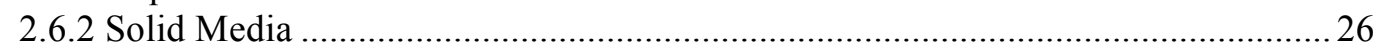

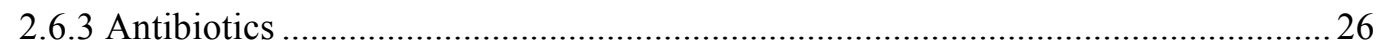

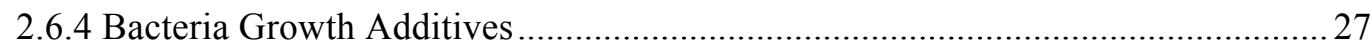

2.7 Growth and Storage of Bacterial Cells .........................................................................27

2.8 Standard Molecular Biology Protocols ...........................................................................28

2.8.1 Isolation of Plasmid DNA ………………………………………………..... 28

2.8.2 Plasmid DNA Quantification ......................................................................... 28

2.8.3 DNA Sequencing.......................................................................................... 28

2.8.4 Agarose Gel Electrophoresis .......................................................................... 28

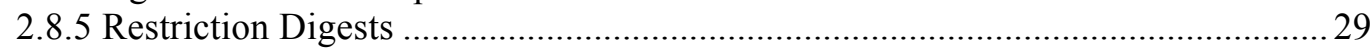

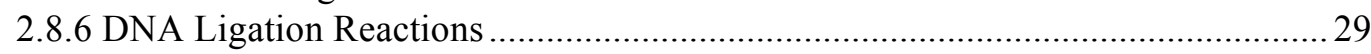

2.8.7 Sodium Dodecyl Sulfate Polyacrylamide Gel Electrophoresis (SDS-PAGE) ........30

2.6.8 Creation of Chemically Competent Cells.............................................................32

2.6.9 Heat-shock Plasmid Transformation of Chemically Competent $E$. coli Cells ........33

2.6.10 Creation of Electrocompetent Cells …………………………………………..... 33 
2.6.11 Electroporation of Electrocompetent E. coli Cells ................................................34

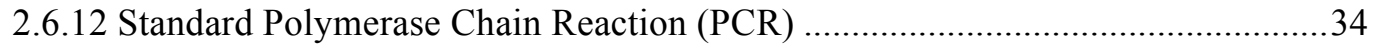

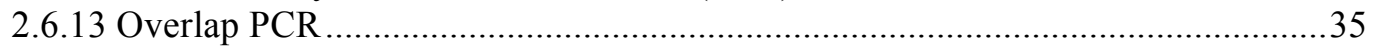

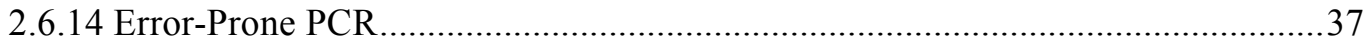

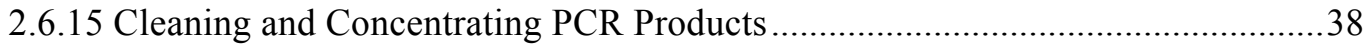

2.7 Negative Selection Directed Evolution Screening Protocol...........................................38

2.8 Fluorescence assays...............................................................................................................38

2.9 Fluorescence Activated Cell Sorting (FACS) of E. coli Cells .......................................39

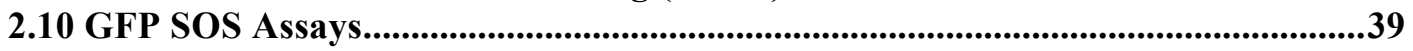

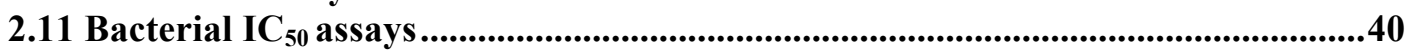

2.12 Growth Inhibition Assays.....................................................................................................41

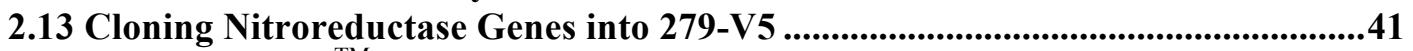

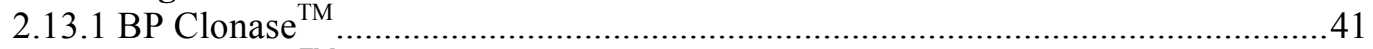

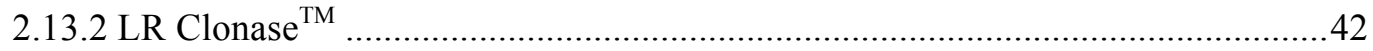

2.14 Mammalian Cell Culture.................................................................................................42

2.14.1 Growth and Maintenance of HEK293 Cells .......................................................4

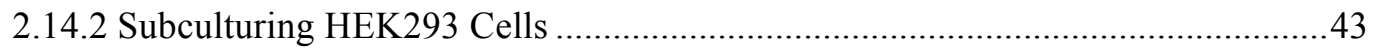

2.14.3 Freezing HEK293 Cells for Long Term Storage ..............................................4 43

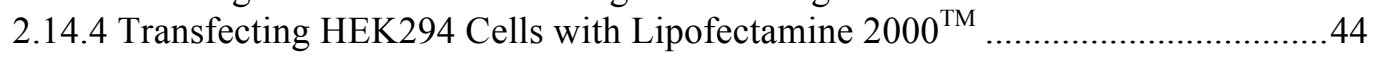

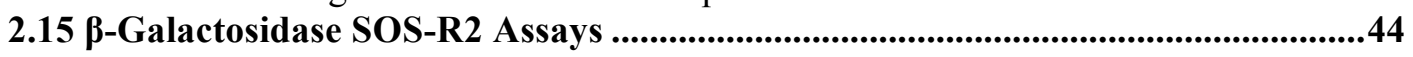

2.16 Imaging Nitroreductase Expression from Colonies Using 4-Nitroimidazole ........46

Chapter 3: Enhancing the Tinidazole Selectivity of Nfs__Ec using Directed

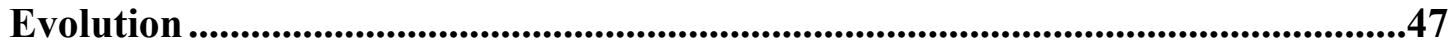

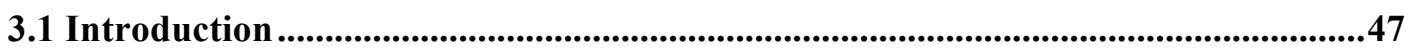

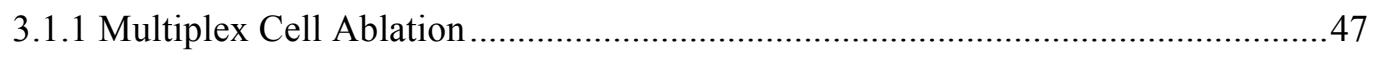

3.1.2 Directed Evolution to Evolve Highly Specific Enzymes ......................................50

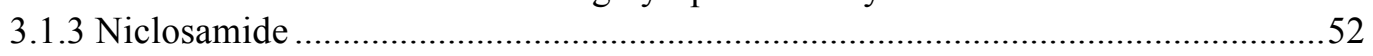

3.1.4 SOS GFP and Growth Inhibition/ $/ \mathrm{IC}_{50}$ Assays .............................................5

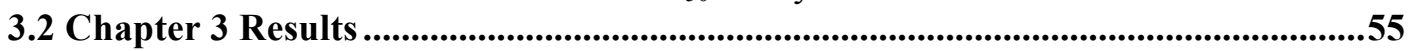

3.2.1 Activity of Wild Type Nitroreductase Enzymes with Metronidazole and Tinidazole

3.2.2 Positive Selection of Active NfsA_Ec 7SM Mutants using Niclosamide..............57

3.2.3 Optimization of Metronidazole Concentrations for Negative Selection .................62

3.2.4 Preliminary Testing of Directed Evolution Parameters .........................................66

3.2.5 NfsA_Ec 7SM Library Screening using Metronidazole and Niclosamide Supplemented

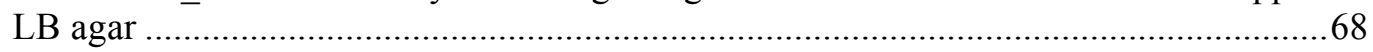

3.2.7 Metronidazole and Tinidazole $\mathrm{IC}_{50}$ values of top 7SM mutants ...............................74

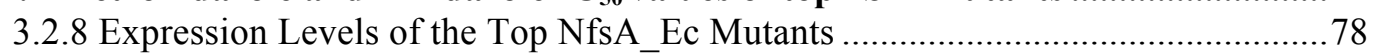

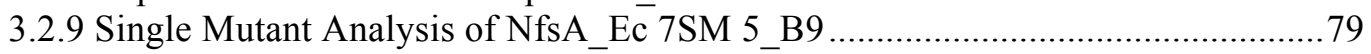

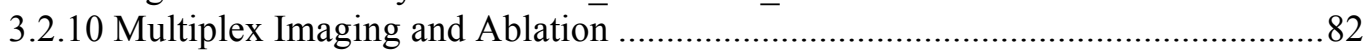

3.2.11 Preparation for Eukaryotic $\mathrm{IC}_{50}$ analysis of the Selected NfsA_Ec 7SM mutants90

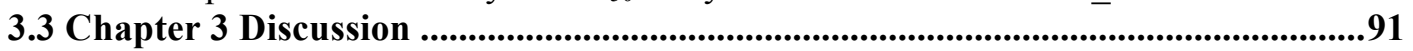

Chapter 4: Enhancing the Tinidazole Selectivity of Nfs__Pp using Directed

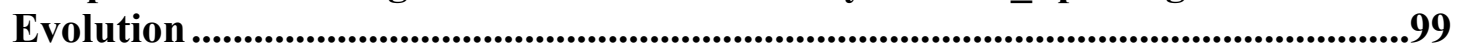

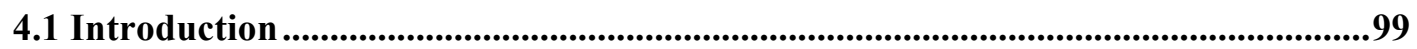

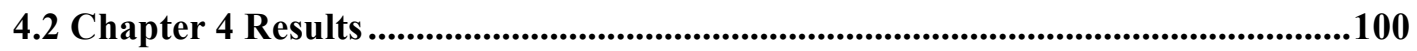

4.2.1 Optimization of Directed Evolution Screen for NfsA_Pp ....................................100

4.2.2 Preliminary Screening of a Random Mutagenesis NfsA_Pp Mutant Library ...... 101

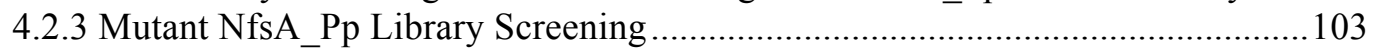

4.2.4 Sequence and Growth Inhibition Analysis of the top NfsA_Pp Mutant ...............105

4.3 Chapter 4 Discussion ...........................................................................................................108

Chapter 5: Metagenomic Discovery of New Nitroreductases ............................110

5.1 Metagenomic Screening using Profluorophore Molecules ...........................................111 
5.1.1 Activation of FSL41 by E. coli W3110 Cells

5.1.2 Increased Expression of pETDUET Plasmids in the E. coli 7TL Strain............... 113

5.1.3 Proof of Concept FACS Enrichment of Nitroreductase Overexpressing Cells.... 116

5.2 Niclosamide Metagenomic Screening

5.3 SOS/ß-Galactosidase Metagenomic Screening

5.4 Metagenomic Screening Using 4-Nitroimidazole

5.4.1 4-Nitroimidazole can Detect the Expression of Nitroreductase Enzymes at a Colony level

5.4.2 Successful Detection of W3110 Cells within a Higher Background of E. coli 7TL cells

5.5 Chapter 5 Discussion

Chapter 6 Research Motivation, Key Findings, and Future Directions ..............133

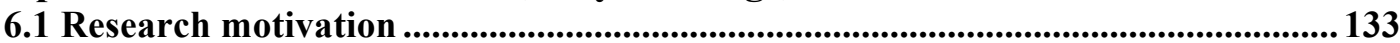

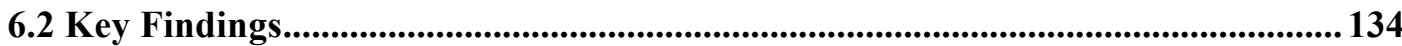

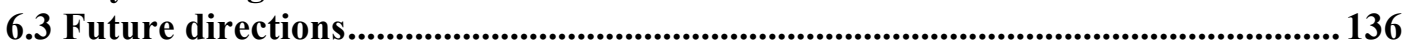

6.3.1 Alternative Prodrug Multiplex Ablation Systems ............................................... 136

6.3.2 Future Directed Evolution Experiments to Enhance Substrate Selectivity ........... 137

7.3.3 Exploring the Mechanistic Basis Behind Substrate Selectivity .......................... 137

6.3.4 Multiplex Imaging using Fluorescent Proteins ................................................ 138

6.3.5 Metagenomic Screening using Chloramphenicol............................................... 138

6.3.7 Metagenomic Screening using Degenerate Nitroreductase Primers .................... 139

6.3.7 Using 4-Nitroimidazole as a Directed Evolution Tool......................................... 139

6.4 Concluding Remarks ................................................................................................................ 141

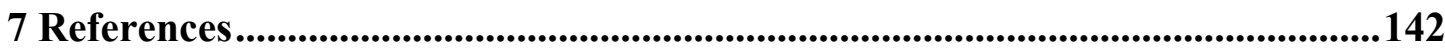

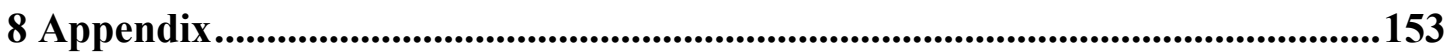




\section{List of Figures}

Figure 1.1 - Structure of the dinitrobenzamide prodrug CB1954 3

Figure 1.2 - Nitroreductase gene-directed enzyme prodrug therapy. $\quad 4$

Figure 1.3 - The GDEPT bystander effect $\quad 5$

Figure 1.4 - Structure of the 5-nitroimidazole nil-bystander prodrug metronidazole 9

Figure 1.5 - Metronidazole Cell Ablation in Zebrafish. $\quad 10$

$\begin{array}{lr}\text { Figure 2.1 - The E. coli Expression Vector pUCX. } & 21\end{array}$

Figure 2.2 - Mammalian expression vector 279-V5. 22

Figure 2.3 - Examples of overlap PCR primers used for single-site mutagenesis of NfsA_Ec. 36

$\begin{array}{ll}\text { Figure 3.1 - Nitroreductase Multiplex Ablation System. } & 48\end{array}$

Figure 3.2 - Structure of the 5-nitroimidazole nil-bystander prodrug tinidazole. 50

Figure 3.3 - Structure of niclosamide. $\quad 52$

Figure 3.4 - Proposed directed evolution screen for enhancing the tinidazole selectivity
of a mutant nitroreductase library expressed from E. coli.

Figure 3.5 - Quantification of prodrug-induced DNA damage using the SOS GFP assay. 54

Figure 3.6 - Metronidazole and tinidazole growth inhibition assays of E. coli
SOS-R4 nitroreductase overexpressing strains.

Figure 3.7 - Viability of E. coli SOS-R4 cells overexpressing NfsA_Ec on niclosamide. 59

Figure 3.8 - Metronidazole and tinidazole induced GFP SOS response in E. coli SOS-R4
overexpressing NfsA_Ec 7SM mutants preselected on niclosamide.

Figure 3.9 - GFP SOS response induced by metronidazole or tinidazole in randomly selected $E$. coli SOS-R4 overexpressing NfsA_Ec 7SM mutants in the absence of niclosamide preselection.

Figure 3.10 - Niclosamide, metronidazole and tinidazole growth inhibition assays of $E$. coli SOS-R4 nitroreductase overexpressing strains.

Figure 3.11 - Replica plating of E. coli SOS-R4 nitroreductase overexpressing strains on metronidazole and tinidazole.

Figure 3.12 - PCR screening of individual colonies from a mixed culture of $E$. coli SOS-R4 pUCX_Empty (49.9\%), NfsA_Ec (49.9\%) and AzoR_Ec (0.2\%) cells plated on LB agar supplemented with metronidazole and niclosamide.

Figure 3.13 - PCR screening of individual colonies from a mixed culture of $E$. coli SOS-R4 pUCX_Empty (49.9\%), NfsA_Ec (49.9\%) and AzoR_Ec (0.2\%) cells plated on unsupplemented LB agar.

Figure 3.14 - Metronidazole and tinidazole induced SOS GFP response in E. coli SOS-R4 overexpressing niclosamide and metronidazole preselected nitroreductase clones.

Figure 3.15 - Metronidazole and tinidazole GFP SOS assays of the top 7SM mutants selected with metronidazole and niclosamide.

Figure 3.16 - Metronidazole and tinidazole growth inhibition assays of E. coli SOS-R4 nitroreductase overexpressing strains.

Figure 3.17 - SDS-PAGE Gel showing relative levels of protein expression of 7SM mutants.

Figure 3.18 - Metronidazole and tinidazole growth inhibition assays of E. coli SOS-R4 NfsA_Ec single mutant overexpressing strains.

Figure 3.19 - Representation of some of profluorophores available in the ACSRC library.

Figure 3.20 - Multiplex imaging and ablation system.

Figure 3.21 - Structures of the nitroaromatic profluorophores FSL76, FSL150, and FSL178.

Figure 3.22 - FSL76 and FSL150 fluorescence assay with E. coli $7 \mathrm{KO}$ cells overexpressing metronidazole-insensitive 7SM NfsA_Ec mutant nitroreductases.

Figure 3.23 - FSL76 and FSL150 fluorescence assay with $E$. coli $7 \mathrm{KO}$ cells overexpressing metronidazole-insensitive 7SM NfsA_Ec mutant nitroreductases.

Figure 3.24 - FSL41 fluorescence assay and UV transillumination of E. coli 7KO cells overexpressing metronidazole-insensitive 7SM NfsA_Ec mutant nitroreductases 
Figure 4.1 - Niclosamide growth inhibition assays of E. coli SOS-R4 nitroreductase overexpressing strains.

Figure 4.2 - Metronidazole and tinidazole induced GFP SOS response in niclosamide preselected E. coli SOS-R4 overexpressing NfsA_Pp mutants.

Figure 4.3 - Metronidazole and tinidazole induced GFP SOS response in $\bar{E}$. coli SOS-R4 overexpressing NfsA_Pp mutant clones preselected with niclosamide and metronidazole.

Figure 4.4 - Protein sequence alignment of NfsA_Ec and the NfsA_Pp mutant Pp_G5.

Figure 4.5 - Metronidazole and tinidazole growth inhibition assays of E. coli SOS-R4 overexpressing either NfsA_Pp or mutated NfsA_Pp enzyme.

Figure 5.1 - FSL41 fluorescence assay on W3110 and E. coli 7TL cells.

Figure 5.2 - SDS-PAGE gel demonstrating induced expression of pETDUET_NfsB_Ec from $E$. coli 7 TL cells.

Figure 5.3 - FSL41 fluorescence assay of post-FACS population of E. coli 7TL pETDUET_NfsB_Ec cells diluted into a culture of $\bar{E}$. coli $\overline{7}$ TL pETDUET empty cells at a 1:999 ratio.

Figure 5.4 - Structure of the prodrug candidate 4-nitroimidazole.

Figure $\mathbf{5 . 5}$ - Reduction of 4-nitroimidazole by nitroreductase overexpressing E. coli $7 \mathrm{KO}$ colonies produces a dark purple pigment.

Figure 5.6 - Reduction of 4-nitroimidazole by W3110 colonies produces a dark purple pigment.

Figure 5.7 - Detection of W3110 colonies from a mixed population of 10\% W3110 and 90\% 7TL colonies using 4-nitroimidazole.

\section{List of Appendix Figures}

Appendix Figure 8.1 - Metronidazole and tinidazole induced growth inhibition response in $E$. coli SOS-R4 overexpressing NfsA_Ec 7SM mutants preselected on niclosamide.

Appendix Figure 8.2 - Metronidazole and tinidazole induced growth inhibition response in $E$. coli SOS-R4 overexpressing NfsA_Ec 7SM mutants lacking niclosamide selection. Metronidazole and tinidazole induced GFP SOS response in $E$. coli SOS-R4 overexpressing niclosamide and metronidazole preselected nitroreductase clones. 


\section{List of Tables}

Table 2.1 - Bacteria strains used in this study. $\quad 20$

$\begin{array}{lr}\text { Table 2.2 - Plasmids used in this study. } & 21\end{array}$

Table 2.3 - Profluorophore used in this study. 23

$\begin{array}{lr}\text { Table 2.4 - Nitroaromatic compounds used in this study. } & 24\end{array}$

$\begin{array}{lr}\text { Table 2.5 - Supplements for Bacterial Growth. } & 27\end{array}$

$\begin{array}{ll}\text { Table 2.6 - Common additives to LB. } & 27\end{array}$

Table 2.7 - Restriction digest reaction components. 29

$\begin{array}{lr}\text { Table 2.8 - Components of typical DNA ligase reactions } & 29\end{array}$

Table 2.9 - Standard PCR thermal parameters. 35

$\begin{array}{ll}\text { Table 2.10 - Overlap PCR thermal parameters. } & 37\end{array}$

Table 2.11 - Mutazyme PCR reaction components. $\quad 37$

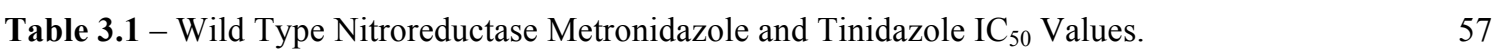

Table 3.2 - Amino acid changes present in the top tinidazole selective NfsA_Ec 7SM
mutants.

Table 3.3 - Metronidazole and tinidazole $\mathrm{IC}_{50}$ assays of $E$. coli SOS-R4
nitroreductase overexpressing strains.

Table 3.4 - Codon changes present in NfsA_Ec 7SM mutant 5_B9. 80

Table 3.5 - NfsA_Ec 7SM mutants selected for transfection into HEK293 cells. 91

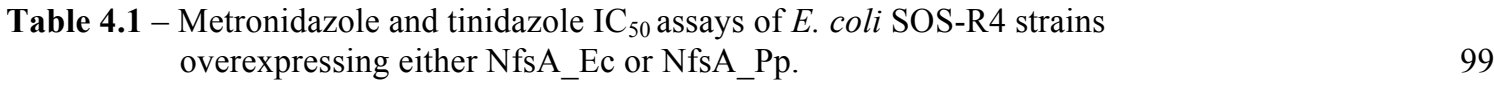

Table 4.2 - Mutations identified in the NfsA_Pp mutant gene Pp_G5. 105 


\section{Abbreviations used in this Thesis}

ACRSC Auckland Cancer Society Research Centre

Amp Amplicillin

APS Ammonium persulflate

bp Base pair

BLAST Basic Local Alignment Search Tool

CB1954 5-(1-Aziridinyl)-2,4-dinitrobenzamide

$\mathbf{d d H}_{2} \mathbf{O}$ double distilled water

DMEM Dulbecco's modified eagle medium

DMSO Dimethyl sulfoxide

eDNA Environmental DNA

FACS Fluorescence-activated cell sorting

FCS Fetal Calf Serum

FMN Flavin mononucleotide

GDEPT Gene-directed enzyme prodrug therapy

GFP Green Fluorescent Protein

IC $_{50}$ Growth of drug required to inhibit normal organism growth by $50 \%$

IPTG Isopropyl $\beta$-D-1-thiogalactopyranoside

Kan Kanamycin

kDa Kilodalton

LB Lysogeny broth

M9 A minimal medium suitable for growth of E. coli

NADH Nicotinamide adenine dinucleotide

NAD(P)H Nicotinamide adenine dinucleotide phosphate

Niclosamide $N$-(2'-chloro-4'-nitrophenyl)-5-chlorosalicylamide

OD $_{600}$ Optical density at $600 \mathrm{~nm}$

PCR Polymerase chain reaction

Puro Puromycin

rpm Revolutions per minute

SDS-PAGE Sodium dodecyl sulfate polyacrylamide gel electrophoresis

SEM Standard error of the mean

Spec Spectinomycin

TEMED Tetramethylethylenediamine

TFBI Transforming buffer I

TFBII Transforming buffer II 


\section{Chapter 1 Introduction}

\subsection{Nitroreductase Enzymes}

Bacterial nitroreductase enzymes are a superfamily of promiscuous flavoprotein oxidoreductases. Nitroreductases can catalyze the reduction of nitro $\left(\mathrm{NO}_{2}\right)$ groups to amine or hydroxylamine functionalities, of which two classes exist: the Type I (oxygeninsensitive) and the Type II (oxygen-sensitive). Type II nitroreductases reduce nitroaromatics though two consecutive transfers of a single electron. The first electron transfer, however, generates an unstable radical intermediate that is rapidly reoxidized in the presence of oxygen (Peterson et al., 1979; Roldán et al., 2008). The second electron transfer, and therefore full nitroreduction, by the nitroreductase therefore only occurs in oxygen-free conditions. In contrast, Type I nitroreductase enzymes, the class considered in this thesis, bypass the unstable intermediate by simultaneously transferring two electrons, enabling nitroreduction to occur regardless of oxygen conditions.

Nitroreductase enzymes typically adopt a homodimeric quaternary structure with an associated flavin (FMN or FAD) prosthetic group (Roldán et al., 2008). The reduction of nitroaromatic substrates occurs through a ping-pong bi-bi mechanism dependent on NADH or NADPH reducing cofactors (de Oliveira et al., 2010a; Roldán et al., 2008). The first step occurs when a reduced nicotinic cofactor enters the active site of the enzyme and reduces the associated flavin. Subsequently, the flavin can then transfer its electrons to reduce a nitroaromatic when it enters the active site. While the nitroreductase enzymes as described are prominent in prokaryotic species, enzymes with nitroreductaselike properties are also found in archaic and eukaryotic species (Roldán et al., 2008).

The physiological role of bacterial nitroreductase enzymes has proven difficult to elucidate. As bacteria and fungi naturally produce nitroaromatic compounds, including multiple antibiotics (Parry et al., 2010), it is conceivable that nitroreductase enzymes evolved as a form of reductive defense against such compounds. For example, the reduced forms of the natural nitroaromatic chloramphenicol have been shown to possess lower levels of antimicrobial activity than the unreduced form (Corbett and Chipko, 1978), Nitroreduction, however, has not been previously documented as a resistance 
mechanism. Additionally, nitroaromatics are a relatively rare functional group to find in natural products (Ju and Parales, 2010). Their scarcity, coupled with a lack of evidence that nitroreduction provides a survival advantage in the presence of such compounds, suggests it was not a major force driving nitroreductase evolution. Several nitroreductases have been hypothesised to play roles in oxidative defense. For example, expression of the nitroreductase enzyme, NfsA, from Escherichia coli (NfsA_Ec), is induced by oxidative stress (Liochev et al., 1999). Similarly, yeast strains with key nitroreductase genes knocked-out have increased sensitivity to oxidative stress (de Oliveira et al., 2010b).

Determining a biological role is further complicated by the substrate promiscuity of nitroreductase enzymes. In addition to nitroaromatics, nitroreductases have demonstrated flavin (Zenno et al., 1998, 1996), chromate (Ackerley et al., 2004; Kwak et al., 2003; Robins et al., 2013), quinone (Nivinskas et al., 2002; Rau and Stolz, 2003), iron (Takeda et al., 2010), uranium (Barak et al., 2006b) and azo dye (Rafii and Cerniglia, 1995) reductase activities. Several mechanisms have been proposed to account for this promiscuity. For instance, it could be the result of the highly water accessible active site typical of nitroreductases, which enables the indirect binding of a range of substrates (Haynes et al., 2002). Additionally/alternatively, it appears nitroreduction is not dependent on any substrate-specific enzyme conformational changes, making the active site highly accessible to structurally diverse substrates (Pitsawong et al., 2014). Regardless of the underlying mechanisms, the substrate promiscuity of nitroreductases enzymes has made them excellent tools for reducing synthetic substrates in biotechnological applications.

\subsection{Applications of Nitroreductase Enzymes}

The reduction catalyzed by nitroreductase enzymes can change the properties of the substrate. Changes to substrate toxicity, in particular, are one of the major areas of research interest. Some compounds that are initially toxic are converted into less toxic forms upon nitroreduction. The hazardous environmental pollutants trinitrotoluene (TNT) and hexavalent chromium, for example, are both converted to less toxic and bioavailable forms upon nitroreductase-catalyzed reduction (Smets et al., 2007; Zhitkovich, 2011). 
The detoxification of these pollutants by nitroreductase enzymes is being developed as a bioremediation strategy. Immobilizing nitroreductases on nanoparticles (Robins et al., 2013), or expressing them from transgenic plants (Hannink et al., 2001; Kurumata et al., 2005; van Dillewijn et al., 2008) have both been tested as a possible means to deliver the enzymes to contaminated sites for bioremediation purposes.

In contrast to substrate detoxification, some non-toxic nitroaromatic compounds are converted (or "activated") into toxic forms by nitroreduction. These compounds are collectively known as prodrugs, an important example being CB1954 (Figure 1.1).

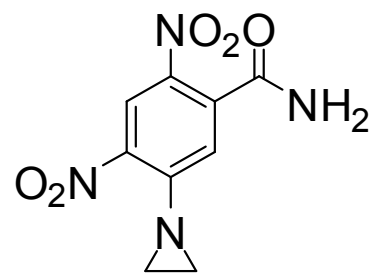

Figure 1.1 - Structure of the dinitrobenzamide prodrug CB1954.

Prior to the reduction of a nitroaromatic prodrug, one or more electronegative $\mathrm{NO}_{2}$ groups draw elections out from the aromatic ring, reducing the reactivity of the compound. Reduction of the $\mathrm{NO}_{2}$ group to a hydroxylamine or amine returns electron density to the aromatic ring restoring reactivity and, in the case of nitroaromatic prodrug, produces a toxic compound (Christofferson and Wilkie, 2009). There is ongoing work developing nitroreductase enzymes and prodrug pairs, including CB1954, for a cancer treatment called gene-directed enzyme prodrug therapy (GDEPT).

\subsubsection{Gene-Directed Enzyme-Prodrug Therapy}

In GDEPT, cancerous tissue is specifically sensitised to a prodrug through targeted expression of a prodrug-activating "suicide" gene. Many different prodrug/suicide gene combinations exist (Patterson et al., 2003); nitroreductase enzymes partnered with nitroaromatic prodrugs, however, are one of the more developed. By specifically targeting cancerous tissue in this manner, the damaging side effects of chemotherapy could be reduced (Patterson et al., 2003). For the therapy to specifically kill cancerous tissue, it is necessary that nitroreductase expression be restricted to cancer cells. Biological gene-delivery vectors including viruses (Bai et al., 2007) and bacteria (Liu et al., 2008) have both been tested as a means to deliver nitroreductase genes to cancer cells 
(Figure 1.2). Viruses and bacteria are promising gene vectors for GDPET as they have a natural affinity for cancerous tissue (i.e., they are oncolytic). The obligate anaerobe Clostridium novyi, for example, forms non-infectious spores in oxygenated environments (Forbes, 2010). Only in oxygen-deprived conditions can the spores germinate and grow. As oxygen is a characteristic of the well-vascularized human body, C. novyi growth is ordinarily restricted. The typically hypoxic tumour microenvironment, formed through disorganized blood vessel formation (Wilson and Hay, 2011), therefore provides an unique opportunity for C. novyi growth (Dang et al., 2001). Alternatively, vectors can be engineered by genetic modification to enhance their oncogenic potential. For example, viruses can be modified to transfect cancer cells with a suicide gene via multiple mechanisms, such as engineering a dependence on cancer specific proteases or receptors for cell entry (Cattaneo et al., 2008; Naik and Russell, 2009). Bacteria can also be engineered to increase their oncolytic potential. For example, deletion of purine synthesis genes from Salmonella typhimurium results in dependence on replicating cancerous tissue for purine (Low et al., 2004).

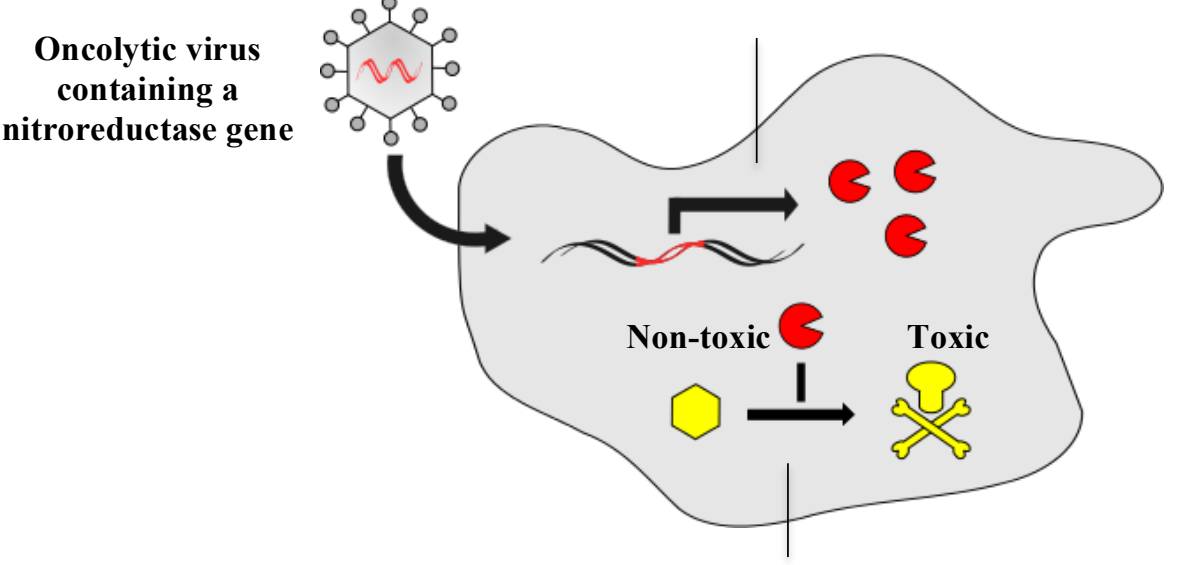

Nitroreductase catalyzed prodrug activation

Figure 1.2 - Nitroreductase gene-directed enzyme prodrug therapy. An oncolytic vector such as a virus is used to deliver a nitroreductase gene into a cancer cell. Expression of the nitroreductase within the cancer cell sensitises it to a nitroaromatic prodrug such as CB1954. Once expressed, the nitroreductase catalyzes the conversion of the initially non-toxic prodrug into a cytotoxic form, killing the host cancer cell.

The limiting factor associated with use of biological gene-delivery vectors is the low efficacy of gene transfection. Unfortunately, the transfection efficiency of a suicide gene into a population of cancerous cells, by any vector, regardless of how oncolytic it is, is highly unlikely to ever be $100 \%$, indeed unlikely to ever exceed 10\% (Dachs et al., 2009). 
It is therefore important that, once activated, the prodrug demonstrates a "bystander effect" and can enter and kill adjacent, untransfected, cells (Figure 1.3).

A)

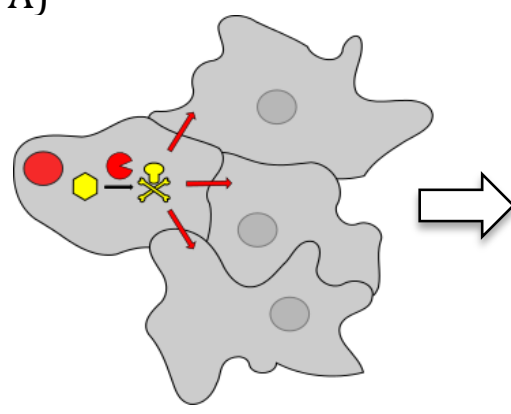

B)

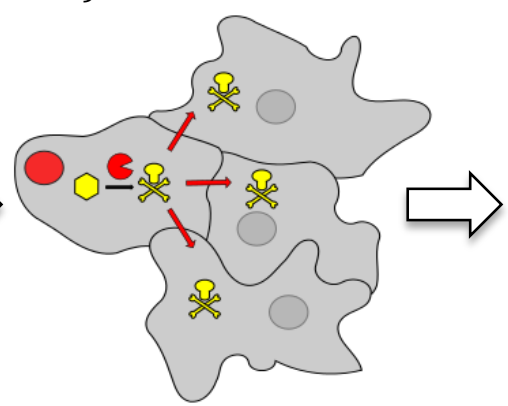

C)

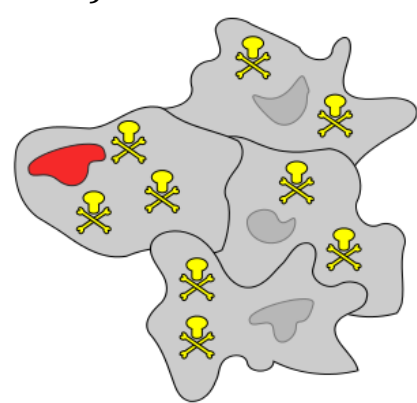

Figure 1.3 - The GDEPT bystander effect. In a GDEPT context, the bystander effect is the transport of an activated prodrug from the cell where it was converted into adjacent tissues. A) A tumour cell expressing a transfected nitroreductase gene (red nucleus) converts a non-toxic prodrug into a toxic form. B) The activated prodrug metabolite can then enter, through active or passive mechanisms, adjacent tumour cells. C) The activated prodrug kills tumour cells that were not expressing a transfected nitroreductase, compensating for the incomplete transfection efficiency.

The majority of nitroreductase GDEPT research has been performed using the oxygeninsensitive NfsB nitroreductase from Escherichia coli (NfsB_Ec) partnered with the prodrug CB1954.

\subsubsection{NfsB_Ec/CB1954 GDEPT}

The prodrug 5-(1-aziridinyl)-2,4-dinitrobenzamide, generically called CB1954, was first synthesized in the 1970s (Sheard et al., 1971) (Figure 1.1). CB1954 contains a 2-NO and a 4-NO $\mathrm{NO}_{2}$ group of which $\mathrm{NfsB}$ Ec reduces in roughly equal ratios (Knox et al., 1992; Race et al., 2007). The more cytotoxic CB1954 metabolite is the 4- $\mathrm{NO}_{2}$ reduction product (Helsby et al., 2003), although the 2- $\mathrm{NO}_{2}$ reduction product possesses a higher bystander effect (Helsby et al., 2004). Following nitroreduction, CB1954 reacts with intracellular thioesters, such as acetyl coenzyme A, to form cytotoxic DNA cross-linking agents (Knox et al., 1991). Activated CB1954 induces apoptosis in both dividing and quiescent cells in a p53 independent manner (Cui et al., 1999; Weedon et al., 2000). Preclinical data was promising, demonstrating that viral transduction of NfsB_Ec into human cancer cell lines sensitised them to CB1954 (Bridgewater et al., 1995; Green et al., 1997; McNeish et al., 1998). Tumour xenograph experiments in mice subsequently 
demonstrated that localized nitroreductase expression was also capable of specifically sensitising cancerous tissue to CB1954 (Lemmon et al., 1997; Lukashev et al., 2005; McNeish et al., 1998; Weedon et al., 2000).

Despite promising preclinical data, when tested in humans $\mathrm{NfsB}$ _Ec partnered with CB1954 failed to produce substantial therapeutic benefit. The notable example was a phase II prostate cancer trial utilizing CB1954 in combination with the NfsB_Eccontaining adenoviral vector CTL102 (Patel et al., 2009). Of the 19 patients that received the treatment, 7 demonstrated a $10 \%$ reduction in the prostate specific antigen (Patel et al., 2009). A reason for the relatively poor response was likely that CB1954 was a poor substrate for NfsB_Ec. The maximum plasma concentration of CB1954 is around $8 \mu \mathrm{M}$ (Patel et al., 2009), well below even the most modest NfsB_Ec $K_{M}$ measurements of 0.9 $\mathrm{mM}$ (Race et al., 2007) - $3.6 \mathrm{mM}$ (Swe et al., 2012). Other experimental measurements having even ranged as high as $17.2 \mathrm{mM}$ (Jarrom et al., 2009). It was therefore unlikely that enough drug could have been physically administered to achieve a therapeutically active dose (Dachs et al., 2009). Nitroreductase GDEPT therefore still has obstacles to overcome before clinical success is possible. However, the central premise of GDEPT, that nitroreductase expression can sensitise a cell population to a prodrug, has proven useful in other fields, namely as a tool to ablate specific tissues in the field of developmental biology.

\subsubsection{Cell Ablation Tools}

Specifically ablating (killing) a cell population in a model organism, typically zebrafish (Danio rerio) is one method of studying the physiological role of those same cells. The phenotypic change that arises following the targeted ablation can provide information on the normal function of cell population (Curado et al., 2008). While traditionally used to study developmental and regenerative processes, strategic cell ablation can also controllably induce disease phenotypes, enabling human diseases to be modeled (Lee et al., 1998). There are two general methods for ablating specific cells: light or chemicalbased strategies (White and Mumm, 2013). 


\subsubsection{Light Ablation Technologies}

Light-based ablation strategies employ targeted lasers that directly kill a specific cell population. Lasers have been used to specifically ablate cell lineages in C. elegans (Bargmann and Avery, 1995), Drosophila (Soustelle et al., 2008) and zebrafish (Roeser and Baier, 2003). An attractive feature of laser ablation is the high level of control and precision over which cells are ablated (Soustelle et al., 2008). Identification of the target population of cells can be assisted by transgenic GFP expression (Roeser and Baier, 2003). Optimizing a laser ablation methodology, however, can be difficult, potentially requiring major investment in expensive optical equipment (White and Mumm, 2013). Uniformly performing the same level of tissue damage between organisms can also be challenging. Additionally, each laser ablation is a challenging and highly timeconsuming procedure, making large sample sizes difficult to process (White and Mumm, 2013). The alternative to light ablation is chemical ablation. Unlike laser ablation, chemical ablation can rapidly and uniformly ablate tissues from multiple organisms simultaneously.

\subsubsection{Chemical Cell Ablation Technologies}

Chemical cell ablation uses compounds that specifically ablate a target cell population (White and Mumm, 2013). The specificity of this strategy can arise either from intrinsic properties of the toxins used, or through tissue modifications that specifically sensitise the tissue to a compound. Intrinsically targeted drugs selectively kill a single tissue by recognising distinguishing characteristics of that tissue. Such compounds have specifically ablated melanocytes (Yang and Johnson, 2006), $\beta$ cells (Soria et al., 2000), hair cells (Harris et al., 2003), and olfactory epithelium cells (Iqbal and Byrd-Jacobs, 2010). In the case of melanocytes, the protoxin 2-morpholinobutyl)-4-thiophenol (MoTP) was converted into a cytotoxic form by the enzyme tyrosinase, an enzyme required for melanin synthesis and therefore a specific melanocyte marker gene (Yang and Johnson, 2006). The major limitation of these compounds is that they can only ablate a single tissue type. An alternative, and more flexible approach, is to modify the target cell population to selectively express a toxic or indirectly toxic gene. 
A commonly used toxic gene product used for cell ablation is diphtheria toxin. Diphtheria toxin is a cytotoxic protein produced by Corynebacterium diphtheriae that inhibits mammalian protein synthesis (Holmes, 2000). The diphtheria toxin is synthesized as a single polypeptide containing two distinct functional units: an A fragment responsible for inhibition of nuclear protein synthesis, and a B fragment responsible for cellular entry. The two fragments must be proteolytically separated within a cell before fragment $A$ is able to inhibit protein synthesis (Collier, 2001). Diphtheria fragment A is highly toxic, to the point where only a single molecule of diphtheria toxin $\mathrm{A}$ is required to kill a cell (Yamaizumi et al., 1978). Expression of diphtheria fragment A from tissue-specific promoters in transgenic mice has enabled the specific ablation of those tissues (Ivanova et al., 2005; Li et al., 1996; Palmiter et al., 1987). Unfortunately, the high toxicity of diphtheria fragment A can also cause unintended cell death and make creation of stable transgenic lines difficult (Curado et al., 2007). Tetracycline-responsive promoters have been used in attempt to control expression of diphtheria fragment A (Lee et al., 1998). However, the inherent leakiness of even tetracycline promoters meant death still occurred prior to gene induction (Keyvani et al., 1999; Paulus et al., 1997). Such unwanted or premature toxicity can be avoided if the transgenic protein is not directly toxic, as in the case of diphtheria toxin A, but like a nitroreductase enzyme, specifically sensitises the cells to a prodrug cytotoxin.

Cell ablation experiments using nitroreductase enzymes have primarily used NfsB_Ec in combination with the GDEPT prodrug CB1954 or the antibiotic metronidazole (White and Mumm, 2013). Tissue specific expression of NfsB_Ec can be achieved through the use of tissue-specific promoter sequences. Transgenic NfsB_Ec expression from tissuespecific promoters has enabled the ablation of mouse astrocytes (Cui et al., 2001), adipocytes (Felmer et al., 2002), luminal cells (Clark et al., 1997), neurons (Isles et al., 2001), and podocytes (Macary et al., 2010) by CB1954. Similarly, rat central nervous system progenitors expressing NfsB_Ec have also been ablated upon CB1954 administration (Kwak et al., 2007). A potential issue with using CB1954 as a cell ablation prodrug, however, is its bystander effect. While a bystander effect is important for GDEPT, in cell ablation experiments the spread of toxic metabolites can cause unwanted off-target cell death (Curado et al., 2008; Felmer et al., 2002; Felmer and Clark, 2004). However, no bystander toxicity was observed when highly vascularized mouse tissues were targeted, believed to be a consequence of rapid dilution of the toxic 
metabolites in the bloodstream (Clark et al., 1997; Isles et al., 2001; Kwak et al., 2007). Moreover, a bystander effect could even be beneficial in some cases for modeling broad tissue injuries (Mathias et al., 2014). CB1954 could have more utility as a specific cell ablation agent in the future by using NfsB_Ec mutants, or orthologous enzymes, that favour production of the 4- $\mathrm{NO}_{2}$ (low bystander) reduction product (Jarrom et al., 2009). Concerns over bystander toxicity can be avoided altogether, however, if the activated prodrug completely lacks bystander effect. The antibiotic metronidazole is an example of "nil-bystander" nitroaromatic prodrug i.e. its toxic reduction product completely lacks a bystander effect, commonly used for cell ablation studies (Lee et al., 2012).

Metronidazole is a 5-nitroimidazole prodrug that is in used clinically as an antibiotic (Edwards, 1980) (Figure 1.4).<smiles>Cc1ncc([N+](=O)[O-])n1CCO</smiles>

Figure 1.4 - Structure of the 5-nitroimidazole nil-bystander prodrug metronidazole.

Reduction of the nitro group on the imidazole ring irreversibly converts metronidazole into a toxic DNA cross-linking agent (Lindmark and Muller, 1976). Generally, toxicity is restricted to invading prokaryotes that express Type I nitroreductase enzymes, or anaerobes that express either Type I or Type II nitroreductases. As human cells tend to be well oxygenated and typically lack Type I nitroreductase enzymes, metronidazole has low toxicity to patients (Golan et al., 2011). Once activated, metronidazole is highly cell entrapped, meaning it lacks a bystander effect (Bridgewater et al., 1997; Curado et al., 2008). Metronidazole in combination with NfsB_Ec has been most widely used to ablate cell lineages in zebrafish models of disease (Figure 1.5). 
Transgenic Juvenile Zebrafish
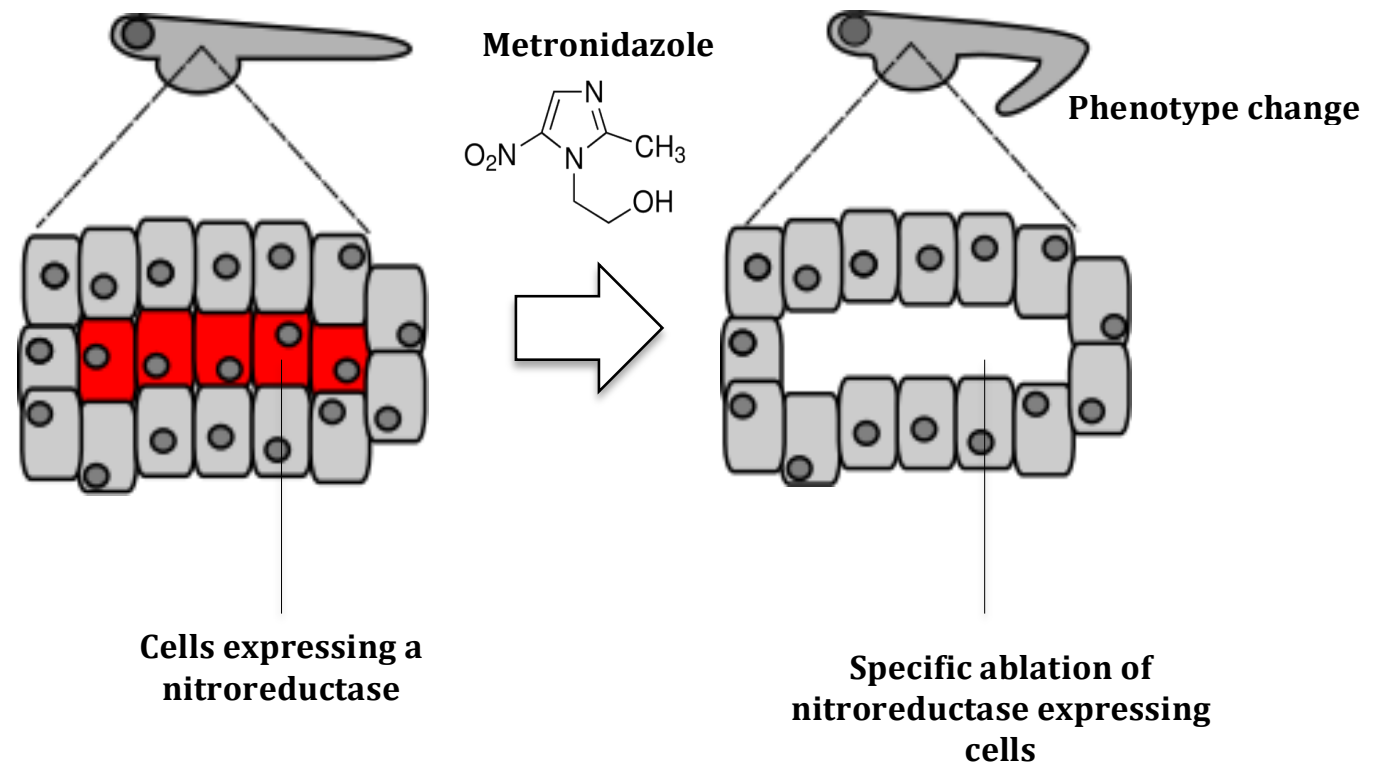

Figure 1.5 - Metronidazole Cell Ablation in Zebrafish. A) A transgenic zebrafish expresses a type I nitroreductase in a single tissue through the use of a tissue specific promoter sequence. When the prodrug metronidazole is administered, it is specifically converted into a toxic form in the nitroreductase expressing tissue. B) Phenotypic changes following the ablation can provide information on the physiological role of the tissue, or on the function of repair processes.

Zebrafish are particularly useful cell ablation models, as they are physiologically similar to humans across a range of attributes, and can even be used to model tissue development and regeneration processes (Lieschke and Currie, 2007). Additionally, as zebrafish embryos are translucent, developmental process can be directly observed under a microscope (Lieschke and Currie, 2007). Tissue specific expression of NfsB_Ec has enabled the metronidazole-mediated ablation of zebrafish podocytes (Zhou and Hildebrandt, 2012), male germ cells (Hsu et al., 2010), oocytes (Hu et al., 2010), rod cells (Montgomery et al., 2010), bipolar cells (Zhao et al., 2009), beta cells (Pisharath et al., 2007), and macrophages (Gray et al., 2011).

Despite the flexibility of NfsB_Ec to activate metronidazole multiple tissues, it is not without its limitations. For example, specificity of cell ablation is highly dependent on a suitable tissue-specific promoter from which to express the nitroreductase transgene (Curado et al., 2008). Additionally, some tissues are less accessible to metronidazole than others, potentially resulting in an incomplete or highly time-delayed ablation (Curado et al., 2008). A larger and more specific problem, with parallels to the 
unsuccessful CB1954 GDEPT clinical trial (Patel et al., 2009), is that metronidazole is also a poor NfsB_Ec substrate (Mathias et al., 2014). High, near lethal, doses of metronidazole are required for cell ablation of NfsB_Ec-expressing cells to occur (Mathias et al., 2014). The standard dosage of metronidazole required for cell ablation is $10 \mathrm{mM}$ (added directly into the fish tank) (Mathias et al., 2014). While $10 \mathrm{mM}$ metronidazole is not lethal to zebrafish embryos during typical $24 \mathrm{~h}$ experiments, over 48 hours it can cause up to $25 \%$ lethality (Mathias et al., 2014). A $10 \mathrm{mM}$ metronidazole dose has also been observed to induce non-specific apoptosis in the superficial telencephalon (Pisharath, 2007). The general toxicity of metronidazole could be decreased if a lower prodrug concentration were be used. A nitroreductase much more active with metronidazole than NfsB_Ec could reduce the concentration required for targeted ablation to occur, reducing off-target lethality and improve ablation experiments. Fortunately, due to the clinical potential of GDEPT, there has been research into developing superior nitroreductase enzymes for the activation of prodrugs.

\subsection{Next Generation Nitroreductase Enzymes}

\subsubsection{Nitroreductases from Culturable Bacteria}

Developing superior enzymes to NfsB_Ec could be achieved by either engineering or discovering new nitroreductase enzymes. Although GDEPT and cell ablation experiments have primarily relied on NfsB_Ec, it is certainly not the only nitroreductase present in nature (Prosser et al., 2013, 2010), or even in E. coli. E. coli possesses another, non-homologous, oxygen-insensitive enzyme capable of efficiently activating CB1954 called NfsA_Ec (Vass et al., 2009). Indeed, NfsA_Ec is around 18-fold more efficient $\left(\mathrm{k}_{\mathrm{cat}} / \mathrm{K}_{\mathrm{M}}\right)$ compared to $\mathrm{NfsB}$ _Ec (Vass et al., 2009) at activating CB1954. The increase in catalytic efficacy translated to also increased sensitivity of ovarian carcinoma SKOV3 cells expressing NfsA_Ec to nitrofuratonin (another nil-bystander prodrug) (Vass et al., 2009).

To readily assess and compare nitroreductase enzymes from a range of species, the Ackerley lab created a "core" plasmid library of 47 (expanded to 58 post-publication) nitroreductase enzymes (Prosser et al., 2013). The genes were cloned from the genomes 
of 21 different bacterial species on the basis of sequence similarity to known nitroreductases, representing 11 distinct enzyme families. All genes were then transformed into E. coli for heterologous expression (Prosser et al., 2013). Screening the library using CB1954 identified the nitroreductases YcnD from Bacillus subtilis and NfsB from Vibrio vulnificus ( $\mathrm{NfsB}_{-} \mathrm{Vv}$ ) as possessing superior $\mathrm{CB} 1954 \mathrm{k}_{\mathrm{cat}} / \mathrm{K}_{\mathrm{M}}$ kinetics to NfsB_Ec (Prosser et al., 2013). Another library member, NfsB from Vibrio harveyi (NfsB_Vh) was much more active with metronidazole ( 20 fold lower bacterial $\left.\mathrm{IC}_{50}\right)$ compared to NfsB_Ec (Condon, 2013). NfsB_Vh also increased metronidazole sensitivity compared to NfsB_Ec when expressed from eukaryotic (HCT-116) cells (Condon, 2013). These data suggest that alternative nitroreductase enzymes offer promise for improving future GDEPT trials and targeted cell ablation studies.

\subsubsection{Metagenomic Discovery of Nitroreductase Enzymes}

To date, all nitroreductase enzymes studied have been purified from culturable bacteria. This approach is inherently limited, however, as an estimated $99 \%$ of all bacteria cannot be cultured in a laboratory setting (Torsvik et al., 1990). The lack of culturability of these organisms is likely due to the inability to accurately replicate factors such as the required nutrient, $\mathrm{pH}$, temperature, and osmotic conditions (Stewart, 2012). While technology advancements have increased the culturability of some bacteria (Ling et al., 2015), metagenomic libraries are the primary method used for studying the genes and gene products of unculturable microorganisms.

A metagenomic library is created by first fragmenting all the DNA present in a environmental sample (referred to as environmental DNA or eDNA) (Piel, 2011). Environmental samples from which the microbial DNA is extracted can include terrestrial soil (Brady et al., 2004), mammalian digestive systems (Hess et al., 2011) or faeces (Xu et al., 2013), insect guts (Warnecke et al., 2007), and seawater (Chu et al., 2008). The fragmented DNA is then cloned in a vector (plasmid or cosmid typically) and transformed into a culturable host such as E. coli (Piel, 2011).

Following metagenomic library creation, two general screening systems exist: functional screens and homology screens (Iqbal et al., 2012). Functional screens detect the 
expression of target genes from the host, via induced phenotypes such as a colour change. In contrast, homology screens use sequence similarity to identify new member of known gene families (Piel, 2011). Screening metagenomic libraries has previously enabled the detection of novel phosphopantetheinyl transferase (Owen et al., 2012), xylose isomerase (Parachin and Gorwa-Grauslund, 2011), polymerase (Simon et al., 2009), and protease (Niehaus et al., 2011) enzymes, to name a few. The genomes of unculturable microorganisms are therefore a promising source for new nitroreductase enzymes in the future.

\subsection{Engineered Nitroreductase Enzymes}

\subsubsection{Directed Evolution}

An alternative to discovering new nitroreductase enzymes is to engineer existing ones using directed evolution. Directed evolution is the process of generating mutants of a given gene, followed by the selection of mutants with improvements in a desired enzymatic activity (Tracewell and Arnold, 2009). Mutations can either be introduced in a random or targeted fashion, the latter generally mutating sites known or believed to be important for catalytic activity (Jäckel et al., 2008). Artificial selection pressures i.e., pressures not typically encountered by the native enzyme, are then applied to select for enzyme variants with desired activities (Cobb et al., 2013).

The starting point for many directed evolution experiments is a promiscuous activity of an enzyme. A promiscuous enzyme activity is one outside the primary activity of the enzyme, and unlikely to have been selected for by nature (Khersonsky and Tawfik, 2010). Promiscuity can come in several forms, such as environment promiscuity - enzymes with activity outside of physiological conditions; substrate promiscuity - enzymes active with a broad range of substrates; and catalytic promiscuity - enzymes capable of catalyzing several distinct chemical transformations (Hult and Berglund, 2007). Enzyme promiscuity may be important from an evolutionary perspective, providing adaptive capabilities to rapid environment changes (Hult and Berglund, 2007). Supporting this view is the observation that promiscuous activities can often be rapidly improved by only

a few genetic changes without affecting native enzyme function (Aharoni et al., 2005; Gould and Tawfik, 2005). Promiscuous activities could therefore enable enzymes to 
rapidly adapt to sudden environmental changes without compromising their native, and often essential, primary activity (Aharoni et al., 2005). Furthermore, the activities of highly promiscuous enzyme are often easier to alter than the activities of enzymes with highly specific functions (Tracewell and Arnold, 2009). Regardless, the high substrate promiscuity of nitroreductase enzymes has made them highly amenable to directed evolution.

\subsubsection{Nitroreductase Directed Evolution}

Directed evolution strategies for improving nitroreductase activity with CB1954 have ranged from rational, utilizing previously solved protein crystal structures, to random mutagenesis approaches. The earliest example of directed evolution to improve prodrug activation by a nitroreductase used the crystal structure of NfsB_Ec to identify the substrate-binding pocket of the enzyme (Grove et al., 2003). Nine amino acid residues surrounding the site were individually targeted for NNN codon mutagenesis, producing nine separate libraries, each with 64 gene iterations apiece. For screening purposes the libraries were chromosomally integrated into $E$. coli $\Delta n f s B$ cells using a bacteriophage vector. Individual E. coli $\Delta n f_{S} B$ lysogens were replica plated on multiple agar plates containing escalating CB1954 concentrations. E. coli $\Delta n f s B$ cells expressing superior NfsB_Ec variants were inviable on lower CB1954 concentrations than a wild-type expressing lysogen. The top mutant (an F142K substitution) sensitised SKOV3 cells to around five-fold lower levels of CB1954 than wild-type NfsB_Ec and possessed 2.4fold improvement in $\mathrm{k}_{\mathrm{cat}} / \mathrm{K}_{\mathrm{M}}$ (Grove et al., 2003; Race et al., 2007). Combining F142K with N71S, another beneficial single mutation identified in the initial screens, synergistically increased the $\mathrm{k}_{\text {cat }} / \mathrm{K}_{\mathrm{M}}$ beyond each mutation alone (Race et al., 2007). However, the combination of beneficial single mutations did not always synergistically improve activity with CB1954 (Jaberipour et al., 2010).

Another directed evolution experiment used the NfsB enzyme FRaseI from the marine bioluminescent bacterium Vibrio fischeri. Unlike its E. coli analogue, FraseI is a highly active FMN reductase capable of supplying $\mathrm{FMNH}_{2}$ to the bioluminescence reaction (Zenno et al., 1994). FRaseI was selected for directed evolution as it exhibited superior CB1954 activity $\left(\mathrm{k}_{\mathrm{cat}} / \mathrm{K}_{\mathrm{M}}\right)$ compared to NfsB_Ec (Swe et al., 2012). Site-saturation 
mutagenesis was performed separately on nine predicted catalytically important codons to produce nine mutagenesis libraries, similar to the method used in Grove et al., 2003. The libraries were screened with CB1954, using DNA damage as a quantitative readout of prodrug activation (Discussed in Section 3.1.4) to identify superior enzyme variants. The seven top mutants all demonstrated improvements in CB1954 $\mathrm{K}_{\mathrm{M}}$ compared to wild type FRaseI. The top mutant identified, a F142W substitution, exhibited an 8.2 fold increase in CB1954 $\mathrm{k}_{\text {cat }} / \mathrm{K}_{\mathrm{M}}$ compared to wild type. An F142 site substitution was also the top NfsB_Ec mutant identified in the Grove et al., 2003 study, demonstrating that catalytically important residues can be conserved within the NfsB family. Also as previously observed with NfsB_Ec (Race et al., 2007), the combination of beneficial single mutations into FRaseI double mutants synergistically enhanced CB1954 activity (Swe et al., 2012).

The DNA damage caused by activated CB1954, like many nitroaromatic prodrugs, induces the bacterial SOS response (Dale et al., 1989). The SOS response is a stress/repair pathway induced by genotoxic damage, specifically fragmented singlestranded DNA (Baharoglu and Mazel, 2014). In addition to inducing a repair network of genes, the SOS response also can also induce chromosomally integrated bacteriophages to enter the lytic cycle and exit their host cell. The SOS-induced lytic cycle was previously exploited to evolve NfsB_Ec enzymes superior at activating CB1954 (Guise et al., 2007). An NfsB_Ec mutant library was created with three catalytically important codons, out of a possible five, randomized by NNN codon mutagenesis $(\sim 1,000,000$ variants). As before, the mutants were packaged into a bacteriophage and integrated into E. coli $\Delta n f_{s} B$. When a mixed culture of mutant E. coli $\Delta n f s B$ lysogens was transiently challenged with a low dose of CB1954, only the most active NfsB_Ec variants converted enough prodrug to induce the phage lytic cycle (Guise et al., 2007). The released phages, containing the nitroreductase mutants, were then collected and subjected to two more identical rounds of CB1954 selection. The top triple mutant identified, T41Q/N71S/F124T, had a measured CB1954 $\mathrm{k}_{\text {cat }} / \mathrm{K}_{\mathrm{M}}$ that was $\sim 45$-fold greater than wild type NfsB_Ec (Jarrom et al., 2009).

Nitroreductases evolved for improving GDEPT have also been beneficial for targeted cell ablation in zebrafish. The NfsB_Ec T41Q/N71S/F124T mutant identified in Guise et al (2007) also possessed superior metronidazole activation (Mathias et al., 2014). When 
expressed from spinal motor neurons, the mutant increased sensitivity to both CB1954 and metronidazole (Mathias et al., 2014). Significant levels of apoptosis were now achievable using the non-lethal dose and challenge period of $10 \mathrm{mM}$ metronidazole for 4 h, respectively (Mathias et al., 2014). However, as the T41Q/N71S/F124T mutant was evolved for superior CB1954 activation, there was no assurance it would show improvements, or even any activity at all, with other substrates. That it also demonstrated superior metronidazole activation was serendipitous, and will no doubt be useful for future ablation experiments. The crossover of superior enzymatic activity to other substrates was also observed with the chromate/nitroreductase YieF from E. coli (YieF_Ec). As mentioned in Section 1.2, nitroreductase enzymes have the potential to be used as chromate bioremediation agents. To enhance its utility as a bioremediation agent, YieF_Ec was modified by directed evolution to improved its Cr(VI) reduction activity (Park et al., 2001). However, several of YieF_Ec mutants with superior Cr(VI) reduction activity also possessed enhanced activity with CB1954 (Barak et al., 2006a). In ongoing work the Ackerley lab has made similar observations regarding the general increase of catalytic activity of nitroreductase mutants with multiple substrates. For example, many nitroreductase mutants selected for improved activity towards the secondgeneration prodrug PR-104A (a dinitrobenzamine mustard) are also more active with metronidazole (Dr. Janine Copp, Dr. Elsie Williams, unpublished). Nitroreductase enzymes evolved for GDEPT or chromate reduction could therefore serve as valuable tools for cell ablation studies using metronidazole, as the T41Q/N71S/F124T mutant demonstrated. Relying on mutants selected with GDEPT prodrugs, however, may inherently restrict activity maxima for the nil-bystander prodrugs used for cell ablation. To date, no directed evolution studies have been performed specifically to improve nitroreductase enzymes for targeted cell ablation. The primary goal of this thesis was to address this deficiency by evolving nitroreductases that were specifically optimized for cell ablation applications.

\subsection{Aims of this Thesis}

There were two major aims of this thesis. The first was to use directed evolution to expand the capabilities of nitroreductase cell ablation studies: Specifically, the development of nitroreductase pairs possessing non-overlapping prodrug selectivities. 
Co-expressing the pair in two different zebrafish tissues would provide temporal control over the ablation of each in isolation, or together simultaneously. The second aim was to expand the range of nitroreductases available to future biotechnological applications, by discovering new nitroreductases from metagenomic libraries and assessing their potential as cell ablation enzymes. 


\section{Chapter 2 Methods}

\subsection{Oligonucleotide Primers}

\subsubsection{Storage of Primers}

All primers used in this study were synthesised by Integrated DNA Technologies ${ }^{\mathrm{TM}}$ (IDT; Coralville, IA, USA) and supplied lyophilized. For long-term storage, primers were resuspended in TE buffer $(10 \mathrm{mM}$ Tris- $\mathrm{Cl} \mathrm{pH} 8.0,0.1 \mathrm{mM}$ EDTA) to a final concentration of $100 \mu \mathrm{M}$ and kept at $-20{ }^{\circ} \mathrm{C}$.. For working stocks, aliquots were diluted in autoclaved, $0.22 \mu \mathrm{m}$ filter-sterilized, distilled and deionised $\left(\mathrm{ddH}_{2} \mathrm{O}\right)$ water to a final concentration of $10 \mu \mathrm{M}$.

\subsubsection{Primers Used}

Underlined sequences indicate restriction sites used for cloning of amplified PCR products.

All primers are written 5' to 3'.

Nitroreductase-specific primers

\begin{tabular}{|c|c|}
\hline NfsA_Ec_Fw & GGCATATGACGCCAACCATTGAAC \\
\hline NfsA_Ec_Rv & GGGTCGACTTAGCGCGTCGCCCAACCCTG \\
\hline AzoR_Ec_Fw & GGGGCATATGAGCAAGGTATTAGTTCTT \\
\hline AzoR_Ec_Rv & GGGGGTCGACTTATGCAGAAACAATGCTGT \\
\hline NfsB_Ec_Fw & GGGCATATGGATATCATTTCTG \\
\hline NfsB_Ec_Rv & GGGGAATTCTTACACTTCGGTTAAG \\
\hline NfsA_Pp_Fw & CCCCCATATGAGCCTTCAAGACGAAG \\
\hline NfsA_Pp_Rv & CTAGGTCGACTCAGCGCAGGCCGAAAC \\
\hline NfsB_Vv_Fw & GGGGCATATGACTATTGTTCAAGCT \\
\hline NfsB_Vv_Rv & GGGGTCGACTTAGATTTCGGTAAAAACAG \\
\hline
\end{tabular}


Gateway Primers

\begin{tabular}{|ll|}
\hline NfsA_EcGW_Fw & GGGGACAAGTTTGTACAAAAAAGCAGGCTTCGAAGGAGATAGAA \\
& CCATGGGCACGCCAACCATTGAACT \\
NfsA_EcGW_Rv & GGGGACCACTTTGTACAAGAAAGCTGGGTCCTAGCGCGTCGCCCA \\
& ACCCTG \\
\hline
\end{tabular}

Primers used for single site mutagenesis of $N f A_{-} E_{C}$

\begin{tabular}{|ll|}
\hline F42C_Fw & GTGCGACGTCCAGTTCCAGTTGCTTGCAGTGCAGTAGCATT \\
F83L_Fw & TCTGGGTGTTCTGTGCCGACCTGAACCGCCATTTACAGATC \\
K222H_Fw & ATATCCGCCGAACAATCATTCATGAAAGCCGCCCATTTAT \\
S224C_Fw & GCCGAACAATCATTAAAGAATGCCGCCCATTTATTCTGGAT \\
F227I_Rv & GGGTCGACTTAGCGCGTCGCCCAACCCTGTTTGTGCAAATAA \\
& TCCAGAATAATATCCAGAATAA \\
F42C_Rv & CAACAGTTGTTCCGCCAGG \\
F83L_Rv & GTCGGCACAGAACACCCAGA \\
K222H_Rv & AATGATTGTTCGGCGGATAT \\
S224H_Rv & TTCTTTAATGATTGTTCGGC
\end{tabular}

Miscellaneous primers

\begin{tabular}{|ll}
\hline pMMB_Fw & GGCTCGTATAATGTGTGG \\
pMMB_Fw & GACCGCTTCTGCGTTCTGAT \\
T7_promoter & TAATACGACTCACTATAGGG \\
T7_terminator & GCTAGTTATTGCTCAGCGG \\
M13_Fw & GTAAAACGACGGCCAG \\
M13_Rv & CAGGAAACAGCTATGAC
\end{tabular}




\subsection{Bacteria Strains Used}

All bacterial strains used in this study are presented in Table 2.1.

\begin{tabular}{|c|c|c|}
\hline Strain & Relevant characteristics & Source \\
\hline W3110 & E. coli $\mathrm{K} 12 \mathrm{~F}^{-} \lambda^{-} \mathrm{rph}-1 \mathrm{IN}(\mathrm{rrnD}-\mathrm{rrnE}) 1$ & Lab stock \\
\hline SOS-R2 & ADA-510 $\Delta n f_{s} A, \Delta n f_{s} B, \Delta a z o R \Delta n e m A \Delta t o l C$ & (Prosser et al., 2013) \\
\hline $6 \mathrm{KO}$ & W3110 $\Delta n f_{s} A, \Delta n f_{s} B, \Delta y i e F, \Delta y c a K, \Delta m d a B, \Delta a z o R$ & (Horvat, 2012) \\
\hline $7 \mathrm{KO}$ & 6KO $\Delta n e m A$ & (Williams, 2013) \\
\hline $7 \mathrm{NT}$ & $6 \mathrm{KO} \Delta$ nemA $\Delta$ tolC & (Copp et al., 2014) \\
\hline $7 \mathrm{TL}$ & $7 \mathrm{NT} \lambda \mathrm{DE} 3$ & Rory Little \\
\hline SOS-R4 & 7NT containing pANODuet (GFP SOS plasmid) & (Copp et al., 2014) \\
\hline BL21 & $\mathrm{F}^{-}$ompT gal dcm lon $h s d S_{B}\left(\mathrm{r}_{\mathrm{B}}^{-} \mathrm{m}_{\mathrm{B}}^{-}\right) \lambda(\mathrm{DE} 3)$ & Novagen \\
\hline DH5 $\alpha$ & supE 44 DlacU 169 (Ø80 lacZ DM5) hsdR17 & Invitrogen \\
\hline
\end{tabular}

Table 2.1 - Bacteria strains used in this study

\subsection{Plasmids}

The plasmids used in this study are presented in Table 2.2. In addition, plasmid maps of the pUCX E. coli expression vector and the 279-V5 mammalian vector are presented in Figure 2.1 and Figure 2.2 respectively. 


\begin{tabular}{|c|c|c|}
\hline Plasmid & Relevant characteristics & Source \\
\hline $\mathrm{pUCX}$ & $\begin{array}{l}\text { Amp }^{\mathrm{r}} \text { E. coli expression vector. tac } \\
\text { promoter, lac operator, pET } 28 \mathrm{a}(+) \\
\text { RBS }\end{array}$ & (Prosser et al., 2010) \\
\hline pETDUET-1 & $\begin{array}{l}\text { Amp }^{\mathrm{r}} \text { E. coli expression vector for co- } \\
\text { expression of two target ORFs. ColE1 } \\
\text { (pBR322) ori, lacI, T7 promoter }\end{array}$ & Novagen \\
\hline pET28a $(+)$ & $\begin{array}{l}\operatorname{Kan}^{\mathrm{r}} \text { expression vector for His6-tagged } \\
\text { enzyme expression. } T 7 \text { promoter }\end{array}$ & Novagen \\
\hline pRSETB & $\begin{array}{l}\text { Amp }^{\mathrm{r}} \text { expression vector for the } \\
\text { Swedish eDNA library. T7 promoter }\end{array}$ & $\begin{array}{l}\text { (Parachin and Gorwa- } \\
\text { Grauslund, 2011) }\end{array}$ \\
\hline pDONR221 & $\begin{array}{l}\operatorname{Kan}^{\mathrm{r}} \text { Entry Vector for } \text { Gateway }^{\mathrm{TM}} \\
\text { recombination cloning }\end{array}$ & Invitrogen \\
\hline $279-V 5$ & $\begin{array}{l}\text { Amp }^{\mathrm{r},} \text { Puro }^{\mathrm{r}} \text { Destination vector for } \\
\text { Gateway recombination cloning. } \\
\text { Mammalian Expression Vector }\end{array}$ & ACSRC (Prosser et al., 2013) \\
\hline pANODuet & $\begin{array}{l}\text { Spec }^{\mathrm{r}} \text { Plasmid containing GFP gene } \\
\text { under control of an SOS (sulA) } \\
\text { promoter. }\end{array}$ & (Copp et al., 2014) \\
\hline
\end{tabular}

Table 2.2 - Plasmids used in this study.

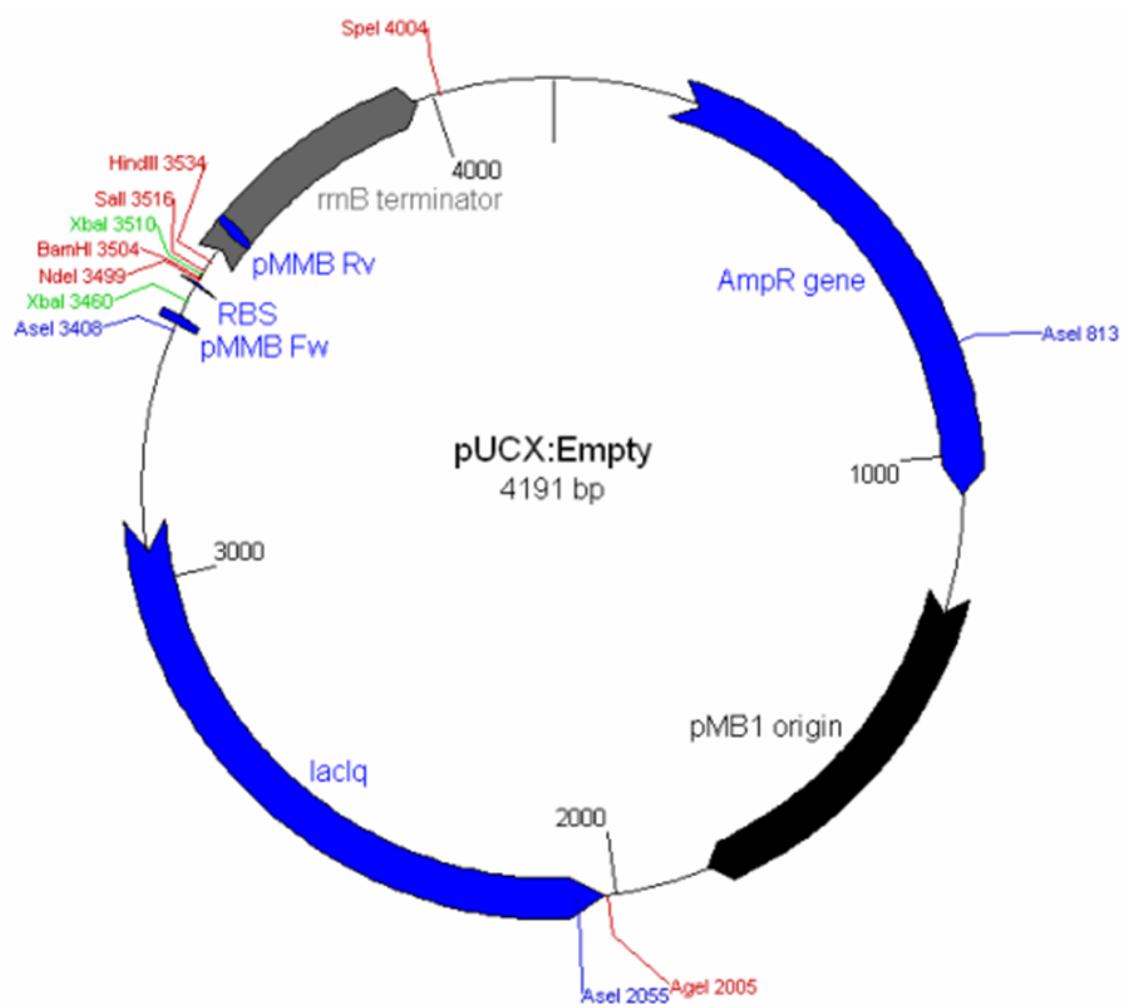

Figure 2.1 - The E. coli Expression Vector pUCX. After cloning a nitroreductase gene into the pUCX multiple cloning site, this vector was used for heterologous nitroreductase expression in E. coli. 


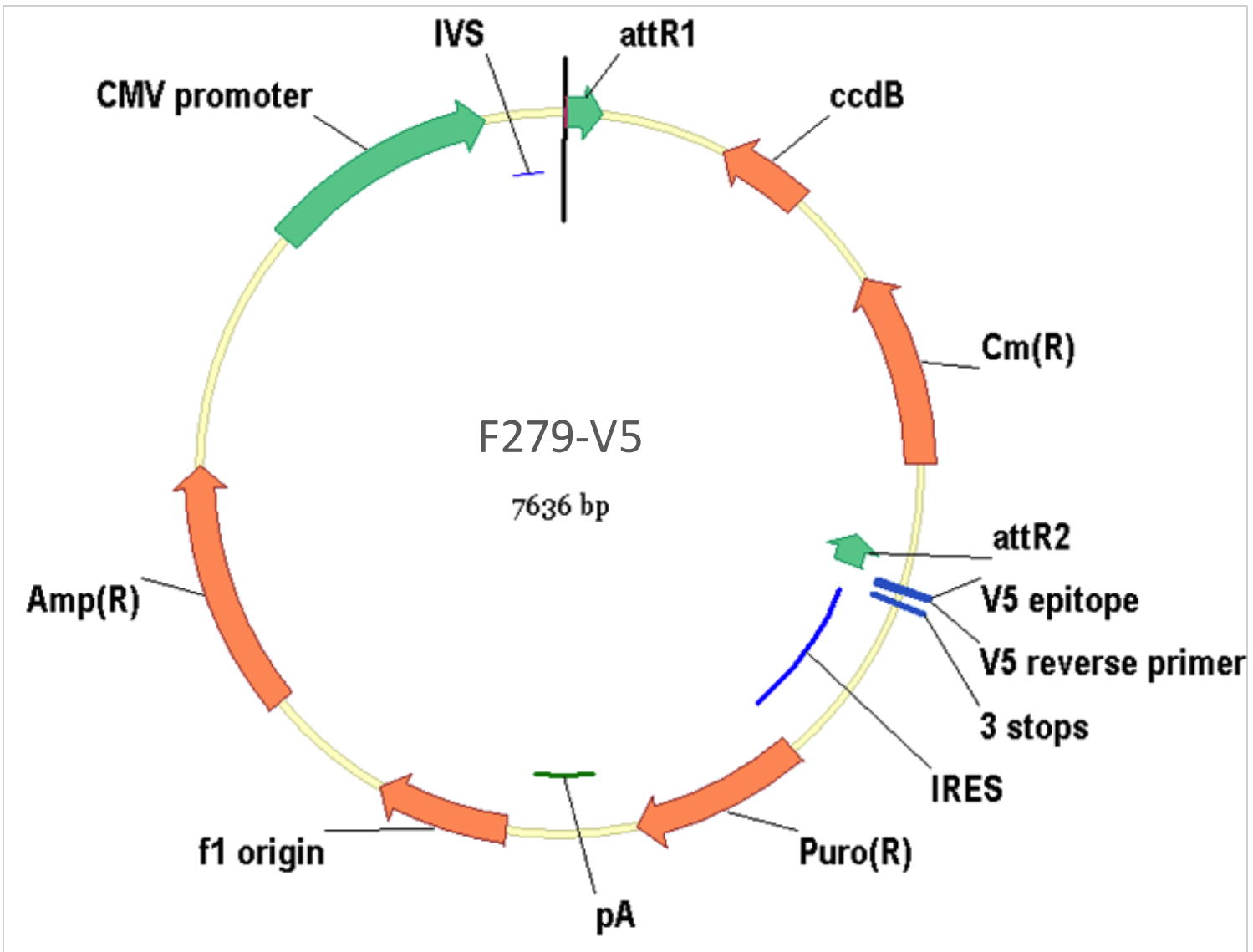

Figure 2.2 - Mammalian expression vector 279-V5. All nitroreductase enzymes to be transfected into HEK293 cells were cloned between the attR1 and attR2 sites using Gateway ${ }^{\mathrm{TM}}$ recombination technology. 


\subsection{Profluorophores}

All nitroroaromatic profluorophores were generously provided by Dr. Jeff Smaill (ACSRC; University of Auckland, New Zealand). Profluorophores were made up from powdered stocks in anhydrous DMSO. Stocks of FSL41, FSL76, and FSL150 were made to $10 \mathrm{mM}$. Stocks of FSL178 were made to $5 \mathrm{mM}$. All profluorophores were stored in aliquots at $-80{ }^{\circ} \mathrm{C}$. Profluorophore structures and excitation/emission wavelengths of the activated (post-nitroreduction) form are presented in Table $\mathbf{2 . 3}$.

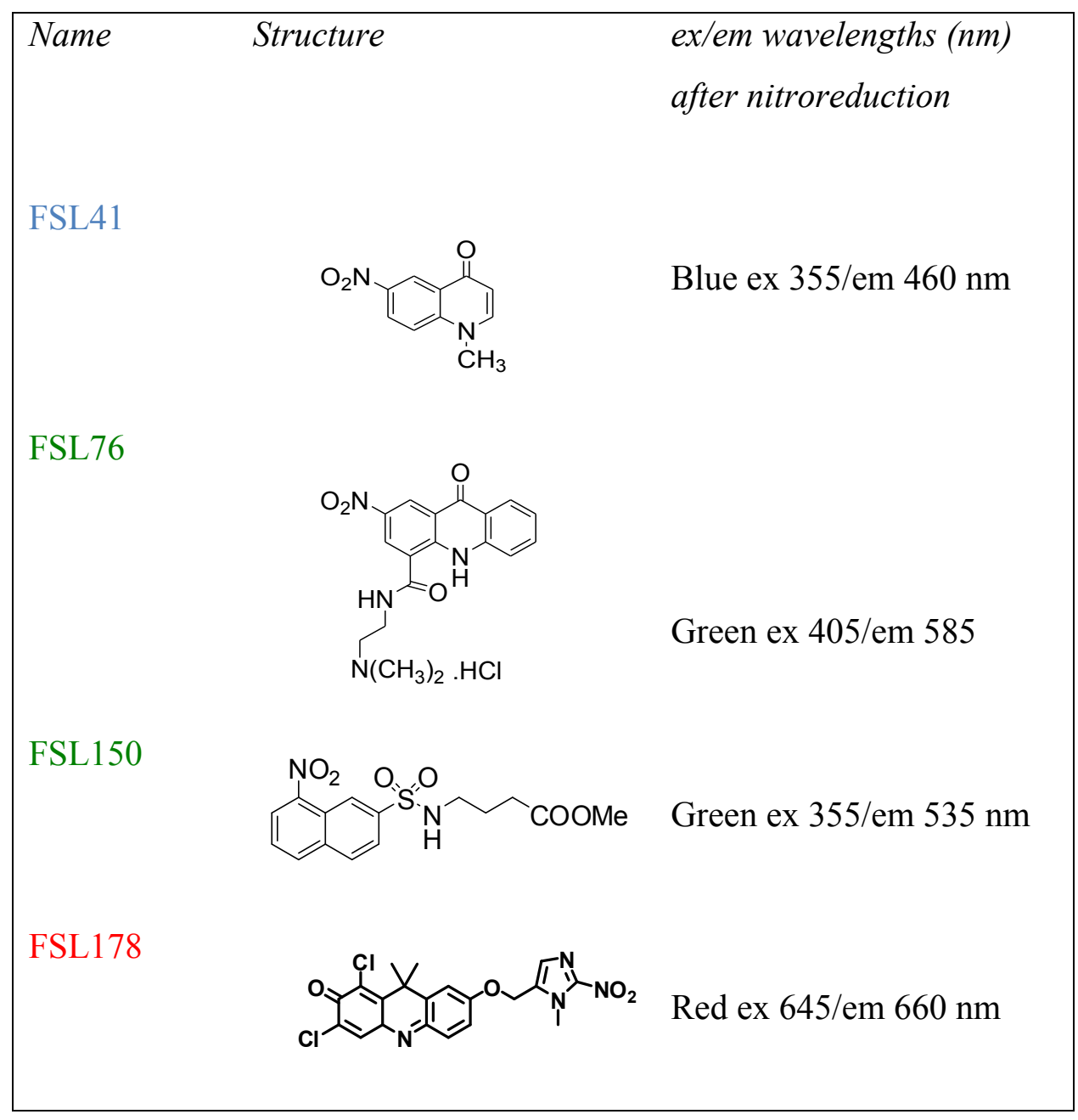

Table 2.3 - Profluorophore used in this study. 


\subsection{Nitroaromatic Compounds}

All prodrugs/compounds (Table 2.4) were made up from powdered stocks in anhydrous DMSO. Stocks of nitroaromatic compounds were stored in aliquots at $-80{ }^{\circ} \mathrm{C}$.

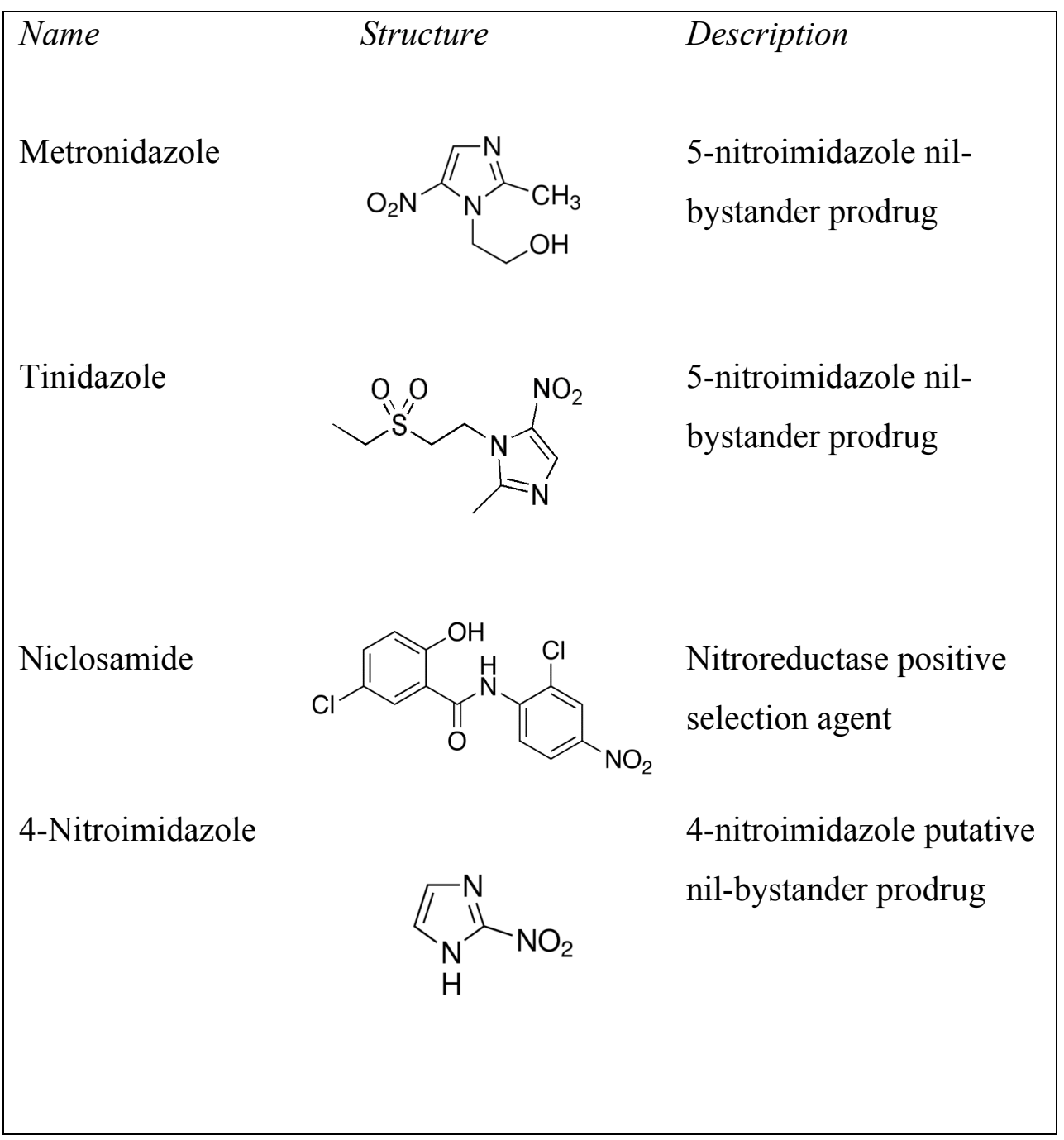

Table 2.4 - Nitroaromatic compounds used in this study. 


\subsection{Growth Media}

Bacteria were either grown in liquid or solid media cultures.

\subsubsection{Liquid Media}

Lysogeny Broth (LB)*

- $1 \%$ Bacterial Peptone (w/v)

- $0.5 \%$ Yeast Extract $(\mathrm{w} / \mathrm{v})$

- $1 \% \mathrm{NaCl}(\mathrm{w} / \mathrm{v})$

*Provided as a premix by Duchefa Biochemie ${ }^{\mathrm{TM}}$ (Haarlem; The Neatherlands)

LB was made up in $\mathrm{ddH}_{2} \mathrm{O}$ to a final concentration of 20 g. $\mathrm{L}^{-1}$ before autoclaving (unless stated otherwise, all autoclaving was conducted at $121{ }^{\circ} \mathrm{C}$ for 30 minutes). Growth supplements were added when the LB had cooled to below $50{ }^{\circ} \mathrm{C}$.

\section{$\underline{\text { SOC Media* }}$}

- Tryptone 20 g.L $\mathrm{L}^{-1}$

- Yeast extract 5 g.L - $^{-1}$

- $\mathrm{KCl} 2.5 \mathrm{mM}$

- $\mathrm{NaCl} 10 \mathrm{mM}$

- $\mathrm{MgCl}_{2} 10 \mathrm{mM}^{* *}$

- Glucose $20 \mathrm{mM}^{* *}$

*Adjusted to $\mathrm{pH} 7.0$ using $\mathrm{NaOH}$

**Filter sterilized in $\mathrm{ddH}_{2} \mathrm{O}$ and added post-autoclave 
M9 Minimal Media*

- $2 \%$ glucose

- $1 \mathrm{x}$ Sigma $^{\mathrm{TM}} \mathrm{M} 9$ salts**

- $2 \mathrm{mM} \mathrm{MgSO}_{4}$

- $100 \mu \mathrm{M} \mathrm{CaCl}_{2}$

*Sterilized with a $0.22 \mu \mathrm{m}$ filter prior to use

**Provided as a premade mixture (Sigma-Aldrich) consisting of:

- 33.9 g. $\mathrm{L}^{-1} \mathrm{Na}_{2} \mathrm{HPO}_{4}$

- 15 g. $\mathrm{L}^{-1} \mathrm{KH}_{2} \mathrm{PO}_{4}$

- 5 g. $\mathrm{L}^{-1} \mathrm{NH}_{4} \mathrm{Cl}$

- $2.5 \mathrm{~g} \cdot \mathrm{L}^{-1} \mathrm{NaCl}$

\subsubsection{Solid Media}

Agar plates for growing bacteria were made by supplementing LB with $1.5 \%(\mathrm{w} / \mathrm{v})$ Sigma Scientific ${ }^{\mathrm{TM}}$ agar, prior to autoclaving. Growth supplements were added to the molten agar once its temperature had dropped below $50{ }^{\circ} \mathrm{C}$. The molten agar was poured into sterile Petri dishes in a sterilized laminar flow hood and left for $30 \mathrm{~min}$ to solidify. Small Petri dishes plates $(90 \mathrm{~mm} \times 15 \mathrm{~mm}$ ) were filled with $15-20 \mathrm{~mL}$ of the molten agar. Large Petri dishes $(150 \mathrm{~mm} \times 25 \mathrm{~mm})$ were filled with $40-50 \mathrm{~mL}$ molten agar. Plates were stored at $4{ }^{\circ} \mathrm{C}$ for up to 3 weeks.

\subsubsection{Antibiotics}

All antibiotics were made up in 1000x stock solution in $\mathrm{ddH}_{2} \mathrm{O}$ and filter-sterilized using a $0.22 \mu \mathrm{m}$ filter. All antibiotic solutions were stored as aliquots at $-20{ }^{\circ} \mathrm{C}$. Final concentrations used are presented in Table 2.5. 


\begin{tabular}{|ll|}
\hline Antibiotic & Final concentration used \\
Ampicillin (Amp) & $100 \mu \mathrm{g} \cdot \mathrm{mL}^{-1}$ \\
Spectinomycin (Spec) & $50 \mu \mathrm{g} \cdot \mathrm{mL}^{-1}$ \\
Kanamycin (Kan) & $50 \mu \mathrm{g} \cdot \mathrm{mL}^{-1}$ \\
Puromycin (Puro) & $1-2 \mu \mathrm{M}$ \\
\hline
\end{tabular}

Table 2.5 - Supplements for Bacterial Growth

\subsubsection{Bacteria Growth Additives}

Stock solutions of IPTG and glucose (Table 2.6) were made up in $\mathrm{ddH}_{2} \mathrm{O}$ and filtersterilized using a $0.22 \mu \mathrm{m}$ filter. Aliquots of IPTG were stored at $-20{ }^{\circ} \mathrm{C}$ while aliquots of glucose were stored at room temperature.

\begin{tabular}{|ll}
\hline Additive & Stock concentration \\
& \\
Isopropyl $\beta$-D-1-thiogalactopyranoside (IPTG) & $100 \mathrm{mg} \cdot \mathrm{mL}^{-1}$ \\
Glucose & $200 \mathrm{mg} \cdot \mathrm{mL}^{-1}$
\end{tabular}

Table 2.6 - Common additives to $\mathrm{LB}$

\subsection{Growth and Storage of Bacterial Cells}

Bacteria were grown in liquid culture at either $30{ }^{\circ} \mathrm{C} 200$ revolutions per minute (rpm) or $37{ }^{\circ} \mathrm{C} 200 \mathrm{rpm}$. The volume of media used made up no more than $1 / 5$ of the total volume of the flask.

Bacteria cultures on agar were grown at $37^{\circ} \mathrm{C}$ in a non-shaking incubator. For long-term storage of bacteria strains, cultures were mixed in a 1:1 ratio with autoclaved $80 \%$ glycerol and stored at $-80{ }^{\circ} \mathrm{C}$. 
Bacteria were recovered from frozen glycerol stocks by removing a small amount of cells using a sterile pipette tip and placing into sterile LB media + appropriate antibiotics. Cells were then grown overnight at $30^{\circ} \mathrm{C} 200 \mathrm{rpm}$.

\subsection{Standard Molecular Biology Protocols}

\subsubsection{Isolation of Plasmid DNA}

All plasmid DNA minipreps were performed using the Geneaid High-Speed Plasmid Mini Kit ${ }^{\mathrm{TM}}$ (Geneaid Biotech Ltd; New Taipei City, Taiwan) as per the manufacturer's instructions. Plasmid DNA was eluted in $50 \mu \mathrm{L}$ of $\mathrm{ddH}_{2} \mathrm{O}$ preheated to $65{ }^{\circ} \mathrm{C}$. Purified DNA samples were stored at $-20{ }^{\circ} \mathrm{C}$.

\subsubsection{Plasmid DNA Quantification}

The purity and concentration of all plasmid minipreps was determined using a Thermoscientific NanoDrop ND-1000 ${ }^{\mathrm{TM}}$ spectrophotometer (Thermo Fisher Scientific; Waltham, MA, USA). Prior to use, the spectrophotometer was blanked with the solvent used to elute the DNA (typically $\mathrm{ddH}_{2} \mathrm{O}$ ).

\subsubsection{DNA Sequencing}

Macrogen Inc. (Seoul, South Korea) performed all Sanger sequencing presented in this thesis. Where possible, at least $100 \mathrm{ng} . \mu \mathrm{L}^{-1}$ of plasmid DNA was sent as a sequencing template.

\subsubsection{Agarose Gel Electrophoresis}

For size quantification of DNA samples, $5 \mu \mathrm{L}$ were run on $1 \%$ agarose gels $(1 \% \mathrm{w} / \mathrm{v}$ agarose in TAE buffer) containing $1 \mu \mathrm{g} . \mathrm{mL}^{-1}$ ethidium bromide. Set gels were submersed in TAE buffer and DNA samples were loaded into each well. Gels were run at $135 \mathrm{~V}$, 
$400 \mathrm{~A}$, for 30 minutes. DNA migration was visualized under UV light. Hyperladder 1 (Bioline; London, UK) was used as a DNA size standard.

\subsubsection{Restriction Digests}

The general reaction setup utilized $\mathrm{NEB}^{\mathrm{TM}}$ (Ipswitch, MA, USA) enzymes and buffers. Prior to digestion, plasmids were heated to $65^{\circ} \mathrm{C}$ for 20 minutes to relieve supercoiling. Reactions were typically set up as shown in Table 2.7.

\begin{tabular}{ll}
\hline Component & Quantity \\
10x CutSmart ${ }^{\mathrm{TM}}$ Buffer & $1 / 10$ final volume \\
Purified DNA & $1-10 \mu \mathrm{g}$ \\
Restriction enzyme(s) & $10 \mathrm{U}$ per $\mu \mathrm{g}$ of DNA \\
$\mathrm{ddH}_{2} \mathrm{O}$ & To dilute the DNA to $20 \mathrm{ng} \cdot \mu \mathrm{L}^{-1}$
\end{tabular}

Table 2.7- Restriction digest reaction components.

Digests were incubated at $37{ }^{\circ} \mathrm{C}$ for 2-3 hours (h) after which restriction enzymes were heat inactivated at $65^{\circ} \mathrm{C}$ for 20 minutes. Digested DNA was stored at $-20{ }^{\circ} \mathrm{C}$.

\subsubsection{DNA Ligation Reactions}

DNA sticky ended ligations were carried out using Bioline ${ }^{\mathrm{TM}}$ (London, UK) T4 DNA ligase. Reactions were set up as shown in Table 2.8 .

\begin{tabular}{ll}
\hline Component & Quantity \\
& \\
$5 \mathrm{x}$ BioLine $^{\mathrm{TM}}$ ligation buffer & $1 / 5$ final volume \\
Digested vector DNA & $100 \mathrm{ng}-200 \mathrm{ng}$ \\
Digested insert DNA & $100 \mathrm{ng}-200 \mathrm{ng}$ \\
$1 \mathrm{U} . \mu \mathrm{L}^{-1} \mathrm{~T} 4$ DNA ligase & $1 \mathrm{U}$ per $200 \mathrm{ng}$ total DNA \\
$\mathrm{ddH} \mathrm{H}_{2} \mathrm{O}$ & To dilute the DNA to $20 \mathrm{ng} \cdot \mu \mathrm{L}^{-1}$
\end{tabular}

Table 2.8 - Components of typical DNA ligase reactions. 
Digested vector and insert DNA were mixed together to achieve a 1:6 molar ratio. Ligation reactions were incubated overnight at $16{ }^{\circ} \mathrm{C}$ prior to storage at $-20{ }^{\circ} \mathrm{C}$.

\subsubsection{Sodium Dodecyl Sulfate Polyacrylamide Gel Electrophoresis (SDS-PAGE)}

\subsubsection{SDS-PAGE Recipe}

All SDS-PAGE stocks were made up in $\mathrm{ddH}_{2} \mathrm{O}$ and autoclaved prior to use.

\section{$\underline{15 \% \text { Loading Gel }}$}

- $14.58 \%$ acrylamide $(\mathrm{w} / \mathrm{v})$

- $0.5 \%$ bis-acrylamide $(\mathrm{w} / \mathrm{v})$

- $\quad 0.375$ M Tris-Cl pH $8.8(\mathrm{w} / \mathrm{v})$

- $\quad 0.1 \% \operatorname{SDS}(\mathrm{w} / \mathrm{v})$
$5 \%$ Stacking Gel

- $\quad 3.87 \%$ acrylamide $(\mathrm{w} / \mathrm{v})$

- $0.107 \%$ bis-acrylamide $(\mathrm{w} / \mathrm{v})$

- $\quad 0.125$ M Tris-Cl pH $6.8(\mathrm{w} / \mathrm{v})$

- $0.1 \% \operatorname{SDS}(\mathrm{w} / \mathrm{v})$

Added into $10 \mathrm{~mL}$ of $15 \%$ loading gel Added into $3 \mathrm{~mL}$ of $5 \%$ stacking gel immediately prior to pouring: immediately prior to pouring:

- $100 \mu \mathrm{L} 10 \%(\mathrm{w} / \mathrm{v})$ ammonium persulfate

- $23 \mu \mathrm{L} 10 \%(\mathrm{w} / \mathrm{v})$ ammonium persulflate

- $6 \mu \mathrm{L}$ tetramethylethylenediamine

- $\quad 3.75 \mu \mathrm{L}$ tetramethylethylenediamine

10x SDS Run Buffer

- $0.144 \%$ glycine $(\mathrm{w} / \mathrm{v})$

- $0.03 \%$ Tris base $(\mathrm{w} / \mathrm{v})$

- $\quad 0.01 \% \operatorname{SDS}(\mathrm{w} / \mathrm{v})$

Commassie Blue Stain

- 2.5 g. $\mathrm{L}^{-1}$ Coomassie Brilliant

Gel Destain Solution Blue

Final concentrations ( $\mathrm{ddH}_{2} \mathrm{O}$ solvent):

- $40 \%$ drum methanol $(\mathrm{w} / \mathrm{v})$

- $45 \%$ absolute ethanol (w/v)

- $10 \%$ acetic acid (w/v) 


\subsubsection{SDS-PAGE Protocol}

For visualisation of bacterial protein expression, 15\% SDS-PAGE gels were cast using a Bio-Rad Protean II ${ }^{\mathrm{TM}}$ apparatus. Roughly $4 \mathrm{~mL}$ of $15 \%$ Separating Gel was applied to the gel cast, covered with $\mathrm{ddH}_{2} \mathrm{O}$ and left to set for $1 \mathrm{~h}$. The water was discarded and the remainder of the gel case was then filled with 5\% Stacking Gel and a gel comb was inserted. The Stacking Gel was then left to solidify for 20 minutes.

\subsubsection{Preparing Bacterial Cultures for SDS-PAGE}

$150 \mu \mathrm{L}$ of overnight bacterial culture was used to inoculate $3 \mathrm{~mL}$ of $\mathrm{LB}+$ relevant antibiotics. These cultures were grown at $37^{\circ} \mathrm{C}, 200 \mathrm{rpm}$ to an $\mathrm{OD}_{600}$ of 0.5 before IPTG was added to a final concentration of $0.5 \mu \mathrm{M}$. Cultures were then grown for an additional $4 \mathrm{~h}$ at $37^{\circ} \mathrm{C}, 200 \mathrm{rpm}$. After the growth period, cultures were normalized to a consistent $\mathrm{OD}_{600}$ by dilution in additional LB media.

$\mathrm{OD}_{600}$-normalized bacteria cultures were added in a 2:1 ratio to $3 \mathrm{x}$ SDS-loading buffer and heated at $95{ }^{\circ} \mathrm{C}$ for 10 minutes. An aliquot of Thermoscientific ${ }^{\mathrm{TM}}$ (NEB; Ipswich, $M A, U S A$ ) unstained protein ladder and samples were boiled at prior to electrophoresis. Gels were run in 1x SDS Run Buffer, at constant voltage (150 V) for approximately $1 \mathrm{~h}$, roughly corresponding to the time at which the SDS load buffer ran off the gel. Protein bands were stained by gentle shaking in Coomassie Blue Stain solution for $1 \mathrm{~h}$. The Coomassie Blue Stain was then removed and replaced with Destain Solution until protein bands were visible (2-3 h). 


\subsubsection{Creation of Chemically Competent Cells}

Solutions required:

TYM

- $2 \%$ tryptone

- $0.5 \%$ yeast extract

- $100 \mathrm{mM} \mathrm{NaCl}$

- $10 \mathrm{mM} \mathrm{MgCl}_{2}$ *
Transforming Buffer II (TFBII)

- $10 \mathrm{mM}$ NaMOPS (pH 7.0)

- $75 \mathrm{mM} \mathrm{CaCl}_{2}$

- $\mathrm{KCl} 10 \mathrm{mM}$

- $15 \%$ glycerol (w/v)

Transforming Buffer I (TFBI)

- $30 \mathrm{mM}$ potassium acetate

- $50 \mathrm{mM} \mathrm{MnCl}_{2}$

- $10 \mathrm{mM} \mathrm{CaCl}_{2}$

- $15 \%$ glycerol $(\mathrm{w} / \mathrm{v})$

All solutions were made up in $\mathrm{ddH}_{2} \mathrm{O}$ and autoclaved prior to use.

*Added post-autoclave

Cell cultures were inoculated overnight at $37^{\circ} \mathrm{C} 200 \mathrm{rpm}$ in $3 \mathrm{~mL}$ of $\mathrm{LB}+$ appropriate antibiotics. The following day, $100 \mu \mathrm{L}$ of the overnight culture was used to inoculate 50 $\mathrm{mL}$ of TYM + relevant antibiotics in a pre-autoclaved $250 \mathrm{~mL}$ conical flask. The culture was grown at $37{ }^{\circ} \mathrm{C} 200 \mathrm{rpm}$ until the $\mathrm{OD}_{600}$ reached $0.3-0.4$. The culture was then placed on ice for 15 minutes before being transferred to a $50 \mathrm{~mL}$ centrifuge tube and centrifuged at $2700 \mathrm{~g}, 2{ }^{\circ} \mathrm{C}$ for 10 minutes. The TYM supernatant was then decanted and the cell pellet resuspended in $0.8 \mathrm{x}$ volume of ice-cold TFBI before being placed on ice again for $2 \mathrm{~h}$. The cell suspension was then centrifuged at $2700 \mathrm{~g}, 2{ }^{\circ} \mathrm{C}$ for 10 minutes and the supernatant decanted. The cell pellet was then resuspended in $0.1 \mathrm{x}$ volume of ice-cold TFBII. The chemically competent cells were snap frozen in $50 \mu \mathrm{L}$ aliquots and stored at $-80{ }^{\circ} \mathrm{C}$. 


\subsubsection{Heat-shock Plasmid Transformation of Chemically Competent $E$. coli Cells}

Chemically competent cells were defrosted on ice before addition of plasmid DNA $(<200$ ng). The cell/DNA mixture was then incubated on ice for 30 minutes. Heat shock was conducted at $43{ }^{\circ} \mathrm{C}$ for 90 seconds, followed by addition of $900 \mu \mathrm{L}$ LB to the cells and incubation at $37^{\circ} \mathrm{C}, 200 \mathrm{rpm}$ for $1 \mathrm{~h}$. Cells were then pelleted by centrifugation at 17,000 $\mathrm{g}$ for 1 minute before being resuspended in $100 \mu \mathrm{L} \mathrm{LB}$. Cells were then plated on LB agar plates (supplemented with appropriate antibiotics for selection of the transformed plasmid). Agar plates were incubated overnight at $37^{\circ} \mathrm{C}$ to promote colony formation.

\subsubsection{Creation of Electrocompetent Cells}

The E. coli strain to be made electrocompetent was grown overnight in LB + appropriate antibiotics. The following day $1 \mathrm{~mL}$ of the overnight culture was grown to an $\mathrm{OD}_{600}$ of 0.5-0.9 in $400 \mathrm{~mL}$ of $\mathrm{LB}+$ appropriate antibiotics. After an $\mathrm{OD}_{600}$ of 0.5 had been reached, the $400 \mathrm{~mL}$ cell culture was split into $50 \mathrm{~mL}$ cultures and placed on ice for 15 minutes. Cultures were then spun at $2700 \mathrm{~g}, 2{ }^{\circ} \mathrm{C}$, for 10 minutes. The supernatant was discarded and cells resuspended in $50 \mathrm{~mL}$ ice-cold $\mathrm{ddH}_{2} \mathrm{O}$. The cell cultures were spun again at $2700 \mathrm{~g}, 2^{\circ} \mathrm{C}$, for 10 minutes. The supernatant was discarded again and the cells were resuspended in $25 \mathrm{~mL}$ sterile ice cold $10 \%$ glycerol (made up in $\mathrm{ddH}_{2} \mathrm{O}$ and autoclaved). Cell suspensions were combined to fit into four $50 \mathrm{~mL}$ tubes. These cultures were spun again at $2700 \mathrm{~g}, 2{ }^{\circ} \mathrm{C}$, for 10 minutes before decanting the supernatant and resuspending in $15 \mathrm{~mL}$ sterile ice cold 10\% glycerol. Cells were spun again at $2700 \mathrm{~g}, 2$ ${ }^{\circ} \mathrm{C}$, for 10 minutes. The supernatant was carefully removed and cells from each tube were resuspended in $150 \mu \mathrm{L}$ of sterile ice cold $10 \%$ glycerol. Cells were snap frozen in 40 $\mu \mathrm{L}$ aliquots and stored at $-80{ }^{\circ} \mathrm{C}$. 


\subsubsection{Electroporation of Electrocompetent $E$. coli Cells}

For each transformation, a $40 \mu \mathrm{L}$ frozen aliquot of electrocompetent E. coli cells was defrosted on ice. Once defrosted, 1-50 ng of purified DNA (eluted in $\mathrm{ddH}_{2} \mathrm{O}$ ) was added to the cells. The DNA and cell mixture was incubated on ice for a further 15 minutes. The cells were then transferred to an ice cold $0.2 \mathrm{~mm}$-gap electroporation cuvette.

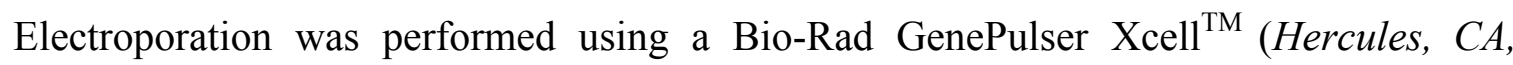
USA). Cells were pulsed at $2500 \mathrm{~V}, 25 \mu \mathrm{F}, 200 \Omega$, followed immediately by the addition of $460 \mu \mathrm{L}$ SOC media at room temperature. Cells were recovered for $1 \mathrm{~h}$ at $37^{\circ} \mathrm{C}, 200$ rpm before plating on an agar containing the appropriate antibiotics.

\subsubsection{Standard Polymerase Chain Reaction (PCR)}

All standard PCR reactions were carried out using Bioline Biomix Red ${ }^{\mathrm{TM}}$ Polymerase Mastermix (London, $U K$ ) with the following reaction components:

Biomix Red ${ }^{\mathrm{TM}}$

- $10 \mu \mathrm{L}(2 \mathrm{x})$ Biomix Red ${ }^{\mathrm{TM}}$

- $8 \mu \mathrm{L} \mathrm{ddH} \mathrm{H}_{2} \mathrm{O}$

- $1 \mu \mathrm{L}$ forward primer $(0.5 \mu \mathrm{M}$ final concentration $)$

- $1 \mu \mathrm{L}$ reverse primer $(0.5 \mu \mathrm{M}$ final concentration $)$

- $\quad 0.5 \mu \mathrm{L}$ template DNA $\left(<100 \mathrm{ng} . \mu \mathrm{L}^{-1}\right)$

When cells on solid media were used as templates for PCR, a small sample of a colony was collected with a sterile pipette tip. 
The standard PCR amplification protocol is displayed in Table 2.9.

\begin{tabular}{|ll|}
\hline Temperature & Time \\
$95{ }^{\circ} \mathrm{C}$ & 2 minutes \\
$95{ }^{\circ} \mathrm{C}$ & 20 seconds \\
$56{ }^{\circ} \mathrm{C}^{*}$ & 20 seconds \\
$72{ }^{\circ} \mathrm{C}$ & 25 seconds per kb amplified \\
$72{ }^{\circ} \mathrm{C}$ & 5 minutes
\end{tabular}

Table 2.9 - Standard PCR thermal parameters.

*The annealing temperature $\left(\mathrm{T}_{\mathrm{A}}\right)$ was increased or decreased depending on the $\mathrm{T}_{\mathrm{M}}$ (melting temperature) of the primers.

For applications requiring a high-fidelity polymerase enzyme, such as molecular cloning, New England BioLabs (NEB) Phusion ${ }^{\mathrm{TM}}$ Polymerase was used as per the manufacturer's instructions.

\subsubsection{Overlap PCR}

Mutant NfsA_Ec genes were created using overlap PCR. For each mutation to be introduced, two internal gene primers were designed. The forward primer contained the desired codon mutation flanked on either side by 15-18 nucleotides homologous to the gene. An example of the forward primer used to generate the NfsA_Ec F82L mutant is displayed in Figure 2.3. The internal reverse primer was the reverse compliment sequence of the nucleotides upstream of the mutant codon in the forward primer. 
- F83L Forward Primer

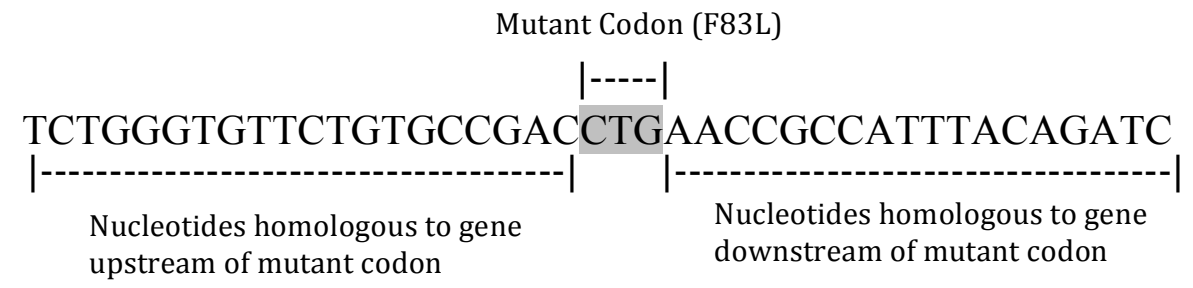

- F83L Reverse Primer:

\section{GTCGGCACAGAACACCCAGA}

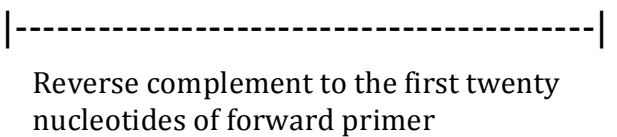

Figure 2.3 - Examples of overlap PCR primers used for single-site mutagenesis of NfsA_Ec.

Two gene fragments were created using gene-specific primers in $10 \mu \mathrm{L}$ Phusion ${ }^{\mathrm{TM}}$ PCR reactions. The first fragment was generated using the internal forward primer and a gene reverse primer, while the second fragment was generated using the internal reverse primer and a gene forward primer. To reduce the chance of wild type plasmid contamination, a low amount $(\sim 100 \mathrm{pg})$ was used as a template. The PCR products were then each treated with $5 \mathrm{U}$ of DpnI for $1 \mathrm{~h}$ at $37^{\circ} \mathrm{C}$ to remove any template plasmid in the solution. The two PCR fragments were purified and mixed together at a 1:1 molar ratio. A complete gene containing the mutant codon was then reconstructed using an overlap PCR protocol (Table 2.10). 


\begin{tabular}{|ll|}
\hline Temperature & Time \\
$98{ }^{\circ} \mathrm{C}$ & 2 minutes \\
$98{ }^{\circ} \mathrm{C}$ & 10 seconds \\
$48^{\circ} \mathrm{C}$ & 30 seconds \\
$72{ }^{\circ} \mathrm{C}$ & 20 seconds \\
Pause & NfsA_Ec specific forward and reverse primers added \\
$98{ }^{\circ} \mathrm{C}$ & 10 seconds \\
$54{ }^{\circ} \mathrm{C}$ & 30 seconds \\
$72{ }^{\circ} \mathrm{C}$ & 20 seconds \\
$72{ }^{\circ} \mathrm{C}$ & 5 minutes \\
\hline
\end{tabular}

Table 2.10 - Overlap PCR thermal parameters.

\subsubsection{Error-Prone PCR}

For the creation of random mutagenesis NfsA_Pp libraries, the GeneMorph ${ }^{\mathrm{TM}}$ II Random Mutagenesis Kit was used. A purified Phusion ${ }^{\mathrm{TM}} \mathrm{PCR}$ product was used as the template DNA in the PCR reaction. To achieve a high mutation rate, 10 ng of DNA (9-16 mutations per $\mathrm{kb}$ ) was added to the $50 \mu \mathrm{L}$ reaction mixture shown in Table 2.11 .

$\begin{array}{ll}\text { Component } & \text { Quantity } \\ \text { 10x Mutazyme II reaction buffer } & 5 \mu \mathrm{L} \\ 40 \mathrm{mM} \text { dNTP mixture } & 1 \mu \mathrm{L} \\ 25 \mu \mathrm{g} \cdot \mu \mathrm{L}^{-1} \text { gene forward primer } & 0.25 \mu \mathrm{L} \\ 25 \mu \mathrm{g} \cdot \mu \mathrm{L}^{-1} \text { gene reverse primer } & 0.25 \mu \mathrm{L} \\ \text { Mutazyme II DNA polymerase } & 1 \mu \mathrm{L}\left(2.5 \mathrm{U} \mathrm{LL}^{-1}\right) \\ \text { Template DNA } & 10 \mathrm{ng} \text { in } 1 \mu \mathrm{L} \mathrm{ddH}_{2} \mathrm{O} \\ \text { ddH } \mathrm{H}_{2} \mathrm{O} & 41.5 \mu \mathrm{L}\end{array}$

Table 2.11 - Mutazyme PCR reaction components.

PCR amplification was performed for 30 cycles using the standard PCR amplification displayed in Table 2.9. 
The Mutazyme PCR products were cloned into a pUCX vector and transformed into electrocompetent SOS-R4 cells for screening. Five mutants were sequences to determine the approximate number of point mutations introduced per gene (from our experience it is lower than predicted by the Mutazyme II parameters).

\subsubsection{Cleaning and Concentrating PCR Products}

PCR products were cleaned and concentrated using Zymo ${ }^{\mathrm{TM}}$ Spin Columns as per the manufacturer's instructions, and eluted in $10 \mu \mathrm{L}$ of sterile $\mathrm{ddH}_{2} \mathrm{O}$ preheated to $65^{\circ} \mathrm{C}$.

\subsection{Negative Selection Directed Evolution Screening Protocol}

Mutant nitroreductase libraries in SOS-R4 were grown overnight from glycerol stocks at $30{ }^{\circ} \mathrm{C}, 200 \mathrm{rpm}$ in LB + Amp + Spec $+0.4 \%$ glucose (LBASG). The following day, 150 $\mu \mathrm{L}$ of the overnight culture was use to inoculate $3 \mathrm{~mL}$ of $\mathrm{LB}+\mathrm{Amp}+\mathrm{Spec}+50 \mu \mathrm{M}$ IPTG $+0.2 \%$ glucose (LBASIG) and grown for $2.5 \mathrm{~h}$ at $30{ }^{\circ} \mathrm{C}, 200 \mathrm{rpm} .250-500 \mu \mathrm{L}$ of this day culture was then plated on a large Petri dish $(150 \mathrm{~mm} \times 25 \mathrm{~mm})$ of agar containing Amp + Spec $+50 \mu \mathrm{M}$ IPTG and supplemented with niclosamide and metronidazole. Niclosamide was added to the molten agar to a final concentration of 0.5 $\mu \mathrm{M}$. When performing metronidazole negative selection, metronidazole was added to molten agar to a final concentration of $800-1000 \mu \mathrm{M}$. Agar plates were then grown overnight at $37^{\circ} \mathrm{C}$. The following day each colony that formed was picked using a sterile pipette tip or toothpick and inoculated into a unique well in a 96 well plate containing 100 $\mu \mathrm{L}$ of LBASG. The plate was grown overnight at $30{ }^{\circ} \mathrm{C}, 200 \mathrm{rpm}$ to made a glycerol stock the following day.

\subsection{Fluorescence assays}

Cultures of E. coli $7 \mathrm{KO}$ cells containing pUCX:nitroreductase plasmids were grown overnight at $30{ }^{\circ} \mathrm{C}, 200 \mathrm{rpm}$ from glycerol stocks in $3 \mathrm{~mL} \mathrm{LB}+\mathrm{Amp}+0.4 \%$ glucose. The following day, $18 \mu \mathrm{L}$ of each overnight culture was added in duplicate to $240 \mu \mathrm{L}$ of filter-sterilized M9 + Amp $+50 \mu \mathrm{M}$ IPTG (M9AI) in a 96-well plate (outer wells excluded). Cell cultures were then grown for $2.5 \mathrm{~h}$ at $37^{\circ} \mathrm{C}, 200 \mathrm{rpm}$. Each duplicate was split into $2 \times 100 \mu \mathrm{L}$ volumes, with $100 \mu \mathrm{L}$ added to $100 \mu \mathrm{L}$ M9AI media containing $20 \mu \mathrm{M}$ profluorophore (thus ensuring a final profluorophore concentration of $10 \mu \mathrm{M}$ ), and 
$100 \mu \mathrm{L}$ added to M9AI containing an equivalent volume of DMSO. Cultures were incubated at $37{ }^{\circ} \mathrm{C} 200 \mathrm{rpm}$ for a further hour after which fluorescence and culture turbidity $\left(\mathrm{OD}_{600}\right)$ were recorded on a EnSpire 2300 Multilabel Reader (Perkin Elmer; Waltham, MA, USA). The excitation and emission wavelengths of the activated profluorophores used in this study are presented in Table 2.3. The fluorescence each culture was divided by its $\mathrm{OD}_{600}$ to normalize by culture turbidity. Relative fluorescence units (RFU) were calculated from the fold difference between the normalized fluorescence values of cultures exposed to the profluorophore, and their respective unchallenged controls. As no background fluorescence was detected for FSL178 cultures, values are presented only as $\mathrm{OD}_{600}$ normalized fluorescence units.

When performing fluorescence assays on E. coli 7TL clones containing metragenomic library fragements, screening was perfrormed in a 384 well plate format identical to that of SOS GFP assays. Profluorophore at $2 \mathrm{x}$ its final concentration was added in place of the prodrug in the challenged wells. Fluorescnce and $\mathrm{OD}_{600}$ were recorded after $1 \mathrm{~h}$ incubation at $37^{\circ} \mathrm{C} 200 \mathrm{rpm}$.

\subsection{Fluorescence Activated Cell Sorting (FACS) of E. coli Cells}

Prior to performing FACS, bacetria cultures were prepared as per a fluorescence assay (Section 2.8). After $1 \mathrm{~h}$ incubation with a profluorophore (FSL41), $10 \mu \mathrm{L}$ of the bacteria culture was added to $990 \mu \mathrm{L}$ of M9 media. Samples were then run through a BD FACS Vantage DiVa flow cytometer (Becton Dickinson; San Jose, CA) using the $355 \mathrm{~nm}$ laser and the 450/50 bandpass with ND1 filter removed from the Forward Scatter Detector.

\subsection{GFP SOS Assays}

SOS-R4 strains transformed with pUCX:nitroreductase plasmids were individually grown overnight in $150 \mu \mathrm{L} \mathrm{LB}+$ Amp + Spec $+0.4 \%$ glucose. The following day $15 \mu \mathrm{L}$ of each overnight culture was used to inoculate $200 \mu \mathrm{L}$ of LB + Amp + Spec $+50 \mu \mathrm{M}$ IPTG $+0.2 \%$ glucose (LBASIG) in 96 well plate format and grown at $30{ }^{\circ} \mathrm{C} 200 \mathrm{rpm}$ for $2.5 \mathrm{~h}$. Following the incubation period, $30 \mu \mathrm{L}$ of each culture was added in duplicate to $30 \mu \mathrm{L}$ LBASIG media containing $2 \mathrm{x}$ the final prodrug concentration, and $30 \mu \mathrm{L}$ of culture was 
added in duplicate to LBASIG media containing an equivalent amount of DMSO, all in the wells of 384 well plates (outer well excluded). Cultures were then incubated for $2.5 \mathrm{~h}$ at $30{ }^{\circ} \mathrm{C} 200 \mathrm{rpm}$. GFP fluorescence $(488 \mathrm{ex} / 510 \mathrm{em})$ and culture turbidity $\left(\mathrm{OD}_{600}\right)$ were then recorded using EnSpire 2300 Multilabel Reader (Perkin Elmer; Waltham, MA, USA). To calculate GFP fold induction values, the raw GFP value of each well was normalized against its $\mathrm{OD}_{600}$ reading. Fold induction was then calculated from the fold difference between the normalized GFP value of each challenged well and its respective unchallenged control. When presented in graphical format, the minimum y-axis value was set to 1.0 (representing no difference in GFP production between the challenged and unchallenged cultures).

\subsection{Bacterial $\mathrm{IC}_{50}$ assays}

Nitroreductase-overexpressing SOS-R4 cells were grown overnight as per the SOS GFP assay. The following day $100 \mu \mathrm{L}$ of each overnight culture was added to $2 \mathrm{~mL} \mathrm{LB}+$ Amp + Spec $+50 \mu \mathrm{M}$ IPTG $+0.2 \%$ glucose (LBASIG) in separate $15 \mathrm{~mL}$ centrifuge tubes. The centrifuge tubes were incubated for $2.5 \mathrm{~h}$ at $30^{\circ} \mathrm{C}, 200 \mathrm{rpm}$. Following the growth period, $40 \mu \mathrm{L}$ of each culture was added to duplicate wells of a 384-well (outer wells excluded) plate already containing $40 \mu \mathrm{L}$ of LBASIG media containing a serial dilution of compound to be tested, including a $0 \mu \mathrm{M}$ concentration, at $2 \mathrm{x}$ final concentration. Culture turbidity $\left(\mathrm{OD}_{600}\right)$ was recorded at $\mathrm{T}_{0}$ and the cells incubated at 30 ${ }^{\circ} \mathrm{C} 200 \mathrm{rpm}$ for an additional $4 \mathrm{~h}$. An $\mathrm{OD}_{600}$ reading was taken after $4 \mathrm{~h}$. Growth of each well was calculated from the difference between the $\mathrm{T}_{0}$ and $\mathrm{T}_{4} \mathrm{OD}_{600}$ values. Growth inhibition caused by prodrug activation was calculated from the difference in growth between challenged cultures and the unchallenged $(0 \mu \mathrm{M})$ control. $\mathrm{IC}_{50}$ values for each nitroreductase-expressing strain (the prodrug concentration at which growth was inhibited by $50 \%$ relative to the unchallenged control) were calculated using the non-linear regression analysis function of GraphPad Prism ${ }^{\mathrm{TM}}$. For calculating the metronidazole or tinidazole $\mathrm{IC}_{50}$ values of wild-type nitroreductases, or the tinidazole $\mathrm{IC}_{50}$ values of NfsA_Ec 7SM mutants, serial dilutions from $800 \mu \mathrm{M}$ were used. For calculating the metronidazole $\mathrm{IC}_{50}$ values of NfsA_Ec 7SM mutants, serial dilutions from $6000 \mu \mathrm{M}$ were used. 


\subsection{Growth Inhibition Assays}

SOS-R4 strains transformed with pUCX:nitroreductase plasmids were grown overnight at $30{ }^{\circ} \mathrm{C}, 200 \mathrm{rpm}$ in $150 \mu \mathrm{L} \mathrm{LB}+\mathrm{Amp}+$ Spec $+0.4 \%$ glucose. The following day, $15 \mu \mathrm{L}$ of each overnight culture was used to inoculate $200 \mu \mathrm{L}$ of LB + Amp + Spec $+50 \mu \mathrm{M}$ $\mathrm{IPTG}+0.2 \%$ glucose (LBASIG). Cultures were then grown for $2.5 \mathrm{~h}$ at $30{ }^{\circ} \mathrm{C} 200 \mathrm{rpm}$. Assays were performed in 384 well plates (outer wells excluded). Following the incubation period, $30 \mu \mathrm{L}$ of each culture was added in duplicate to $30 \mu \mathrm{L}$ LBASIG media containing $2 \mathrm{x}$ the final prodrug concentration and $30 \mu \mathrm{L}$ LBASIG media containing an equivalent volume of DMSO. A $\mathrm{T}_{0} \mathrm{OD}_{600}$ reading was taken. Cultures were incubated for a further $3 \mathrm{~h}$ at $30^{\circ} \mathrm{C}, 200 \mathrm{rpm}$, and the $\mathrm{T}_{3} \mathrm{OD}_{600}$ reading was taken. Growth of each well was calculated from the difference between the $T_{3}$ and $\mathrm{T}_{0} \mathrm{OD}_{600}$ values. Growth inhibition was calculated from the relative difference in growth between challenged cultures and their respective unchallenged controls.

\subsection{Cloning Nitroreductase Genes into 279-V5}

NfsA_Ec 7SM mutants were cloned into a 279-V5 (ACSRC, New Zealand) vector using the Invitrogen Gateway ${ }^{\mathrm{TM}}$ system. Each NfsA_Ec 7SM mutant was first amplified using NfsA_Ec Gateway ${ }^{\mathrm{TM}}$ primers. The Gateway ${ }^{\mathrm{TM}}$ primers introduced a mammalian Kozak consensus sequence and a Shine-Dalgarno bacterial consensus sequence. They also added an additional glycine after each ATG start codon and converted all stop codons to TAG.

\subsubsection{BP Clonase ${ }^{\mathrm{TM}}$}

Each nitroreductase Gateway PCR product was cloned into the entry vector $\mathrm{pDONR}^{\mathrm{TM}} 221$ (Invitrogen Corporation, Carlsbad, CA, USA). Recombination was achieved by mixing $150 \mathrm{ng}$ of $\mathrm{pDONR}^{\mathrm{TM}} 221$ with 50 fmol of Gateway ${ }^{\mathrm{TM}}$ PCR product. The DNA mixture was then added to $1 \mu \mathrm{L}$ of Gateway BP Clonase ${ }^{\mathrm{TM}}$ II enzyme mix (Invitrogen) and TE buffer ( $\mathrm{pH} 8.0)$ to a final volume of $10 \mu \mathrm{L}$. The mixture was incubated overnight at $25{ }^{\circ} \mathrm{C}$. After the overnight incubation, $1 \mu \mathrm{L}$ of proteinase $\mathrm{K}(2$ $\mu \mathrm{g} . \mu \mathrm{L}^{-1}$ ) was added and the reaction mixture incubated at $37{ }^{\circ} \mathrm{C}$ for 10 minutes. $5 \mu \mathrm{L}$ of 
the BP Clonase ${ }^{\mathrm{TM}}$ DNA mixture was transformed into chemically competent DH5 $\alpha$ cells and selected on agar plates containing Kan. Colonies were analyzed for the presence of pDONR $^{\mathrm{TM}} 221$ vector containing an NfsA_Ec 7SM mutant by PCR reaction using M13 Fw and NfsA_Ec Rv primers. Colonies that tested positive were carried forward to the LR Clonase $^{\mathrm{TM}}$ step.

\subsubsection{LR Clonase ${ }^{\mathrm{TM}}$}

The final step was recombination of the nitroreductase gene from the $\mathrm{pDONR}^{\mathrm{TM}} 221$ entry vector into the $279-\mathrm{V} 5$ destination vector. The recombination was performed using Gateway LR Clonase $^{\mathrm{TM}}$ II enzyme mix (Invitrogen Corporation, Carlsbad, CA, USA). 1 $\mu \mathrm{L}$ of LR Clonase ${ }^{\mathrm{TM}} \mathrm{II}$ enzyme mix was added to $150 \mathrm{ng}$ of $\mathrm{pDONR}^{\mathrm{TM}} 221$ :nitroreductase and $150 \mathrm{ng}$ of $279-\mathrm{V} 5$, made up to $10 \mu \mathrm{L}$ in TE buffer (pH 8.0). The mixture was incubated overnight at $25^{\circ} \mathrm{C}$. The following day, as with the BP Clonase ${ }^{\mathrm{TM}}$ step, $1 \mu \mathrm{L}$ of proteinase $\mathrm{K}\left(2 \mu \mathrm{g} \cdot \mu \mathrm{L}^{-1}\right)$ was added and the reaction mixture incubated at $37{ }^{\circ} \mathrm{C}$ for 10 minutes. $5 \mu \mathrm{L}$ of the mixture was then transformed into chemically competent DH5 $\alpha$ cells and plated on agar supplemented with Amp. The following day colonies were screened for recombination of the nitroreductase gene into 279-V5 using T7 Fw and NfsA_Ec Fw primers. Colonies that tested positive had their 279-V5 plasmid sequenced to confirm the cloning had occurred correctly.

\subsection{Mammalian Cell Culture}

\subsubsection{Growth and Maintenance of HEK293 Cells}

All work with HEK293 cells was performed in a laminar flow hood cleaned with $70 \%$ ethanol and irradiated with ultraviolet light for 15 minutes. All solutions to come in contact with cells were preheated to $37{ }^{\circ} \mathrm{C}$. Adherent HEK293 cells were generally grown in Dubecco's Modified Eagle Medium (DMEM) containing 4.5 g.L L $^{-1}$ D-glucose, L-glutamine, $110 \mathrm{mg} . \mathrm{L}^{-1}$ sodium pyruvate and supplemented with $10 \%$ inactivated fetal calf serum (FCS), and 1\% Penicillin Streptomycin (Pen Strep). Cells were typically grown in $6 \mathrm{~mL}$ of DMEM in a T25 tissue culture flask at $37{ }^{\circ} \mathrm{C}$ under $5 \% \mathrm{CO}_{2}$. Half the DMEM in the flask was replaced with fresh DMEM every few days. In cases where a 
high number of dead cells were present, such as after a subculturing, all the media was aspirated and replaced.

\subsubsection{Subculturing HEK293 Cells}

Cells were subcultured once they had reached $75-80 \%$ confluency in the culture flask. Media was aspirated and cells were washed with Dubecco's Phosphate Buffered Saline heated to $37^{\circ} \mathrm{C} .1 \mathrm{~mL}$ Trypsin/EDTA (Life Technologies, Carlsbad; CA, USA) was used to stop the cells adhering to the bottom of the culture tissue. After trypsin was added, cells were incubated at $37{ }^{\circ} \mathrm{C}$ under $5 \% \mathrm{CO}_{2}$ for no more than 5 minutes. Cells were viewed under a light microscope at $4 \mathrm{x}$ magnification to confirm they had detached from the culture flask. In cases where trypsin had not resulted in full detachment, the culture flask was lightly tapped on a solid surface to completely dislodge cells. To neutralise the trypsin, $3 \mathrm{~mL}$ of $10 \%$ FCS containing DMEM was then added to the culture flask. The cell suspension was collected and spun at $300 \mathrm{~g}$ for 5 minutes at room temperature to pellet the cells. The supernatant was decanted and the cell pellet was resuspended in 1 $\mathrm{mL}$ of DMEM. Cells were counted by mixed equal volume of resuspended cell culture with $0.4 \%$ trypan blue viability staining solution. $\mathrm{An}_{2} \mathrm{H}_{2} \mathrm{O}$ sealed haemocytometer was loaded with $8 \mu \mathrm{L}$ of mixture and cell density was calculated as per manufacturer's instructions. Generally, 250,000 - 500,000 live cells were added into a new T25 flask containing $6 \mathrm{~mL}$ fresh DMEM media.

\subsubsection{Freezing HEK293 Cells for Long Term Storage}

To freeze cell lines, cells that had reached $75-80 \%$ confluence were washed and trypsinised as described in Section 2.14.2. After cells were spun down and the supernatant decanted, cell were resuspended in $1 \mathrm{~mL}$ of FCS free, Pen Strep free, DMEM. The cells were diluted to $2 \times 10^{6}$ cells $\mathrm{mL}^{-1}$ and $500 \mu \mathrm{L}$ was added to a cell culture BioStor $^{\mathrm{TM}}$ cryotube (National Scientific Supply Company Inc; Claremont, CA). To the same cryotube, $500 \mu \mathrm{L}$ of ice cold $2 \mathrm{X}$ freeze media was added (unsupplemented DMEM containing 40\% FCS, 20\% DMSO, and 2\% Pen Strep). Cells were mixed with the freeze media before the cyrotube was added to an isopropanol containing Thermo Scientific Mr. Frosty ${ }^{\mathrm{TM}}$ (Thermo Fisher Scientific; Waltham, MA, USA) and placed at -80 
${ }^{\circ} \mathrm{C}$ overnight. The following day the frozen cryotube was transferred to a liquid nitrogen Dewar.

\subsubsection{Transfecting HEK294 Cells using Lipofectamine 2000 ${ }^{\mathrm{TM}}$}

To prepare for HEK293 cell transfection, 500,000 cells were added to a $35 \mathrm{~mm}$ cell culture dish in $2 \mathrm{~mL}$ of DMEM media. To encourage an even distribution of cells, the culture dish was left in the laminar flow for 5 minutes before returning to the $37{ }^{\circ} \mathrm{C} 5 \%$ $\mathrm{CO}_{2}$ incubator overnight. For ease of transport, these cell culture dishes were stored in standard $90 \mathrm{~mm}$ x $15 \mathrm{~mm}$ Petri dishes. Prior to performing the transfection the media was aspirated and the cells were washed with 1x PBS. $2 \mathrm{~mL}$ of media lacking Pen Strep was then added back onto the cells.

All Gateway-cloned nitroreductase $279-\mathrm{V} 5$ vectors were purified in sterile $\mathrm{ddH}_{2} \mathrm{O}$ and quantified prior to transfection. For each transfection, $2 \mu \mathrm{g}$ purified 279-V5 was incubated with $250 \mu \mathrm{L}$ of Opti-Mem ${ }^{\mathrm{TM}}$ reduced serum media for five minutes at room temperature. In parallel, $5 \mu \mathrm{L}$ of Lipofectamine $2000^{\mathrm{TM}}$ (Thermo Fisher Scientific; Waltham, MA, USA) was also incubated in $250 \mu \mathrm{L}$ of Opti-Mem ${ }^{\mathrm{TM}}$ for 5 minutes. The two tubes were then combined and incubated together for 20 minutes at room temperature. After this incubation step, the whole mixture was carefully added into the dish containing the cells in Pen Strep free media. The culture dish was then returned to the $37{ }^{\circ} \mathrm{C} 5 \% \mathrm{CO}_{2}$ incubator. Cells were left to recover for several days, changing the media regularly to remove dead cells, before being grown in DMEM supplemented with $1 \mu \mathrm{M}$ puromycin. Cells containing the $279-\mathrm{V} 5$ plasmid were selected over multiple subculture cycles in escalating concentrations of puromycin (highest concentration used was $2 \mu \mathrm{M}$ ) for several weeks. Once a stable puromycin resistant population had been generated, cells were frozen down as described in Section 2.14.3.

\subsection{5 $\beta$-Galactosidase SOS-R2 Assays}

Stocks of X-gal (5-bromo-4-chloro-3-indolyl-beta-D-galactopyranoside) were made up to $20 \mathrm{mg} \cdot \mathrm{mL}^{-1}$ in anhydrous DMSO and stored at $-20^{\circ} \mathrm{C}$ away from direct light. 
When performing $\beta$-galactosidase assays using SOS-R2 cells to assess its potential as a metagenomic screening method, the following concentrations of $\mathrm{X}$-gal were used (Bioline, London, UK). X-gal was supplemented into agar containing Amp $+0.1 \mathrm{mM}$ IPTG, to a final concentration of $20 \mu \mathrm{g} \cdot \mathrm{mL}^{-1}$. When included in molten top agarose, $\mathrm{X}$ gal was added to a final concentration of $270 \mu \mathrm{g} \cdot \mathrm{mL}^{-1}$. The top agarose was allowed to cool before plates were incubated at $37^{\circ} \mathrm{C}$. 


\subsection{Imaging Nitroreductase Expression from Colonies Using 4- Nitroimidazole}

Solutions required:

$\underline{\text { Top Agarose }}$

- $5 \mathrm{mg} \cdot \mathrm{mL}^{-1} \mathrm{NaCl}$

- $0.6 \%(\mathrm{w} / \mathrm{v})$ agarose

Made up in a suitable volume $\mathrm{ddH}_{2} \mathrm{O}$ and autoclaved before use

Top agarose containing the prodrug 4-nitroimidazole was used to detect the expression of nitroreductase enzymes from single colonies. Stocks of $500 \mathrm{mM} \mathrm{4-} \mathrm{nitroimidazole} \mathrm{were}$ diluted in molten top agarose to a final concentration of 5-20 mM. An equivalent volume of DMSO was also added to increase the solubility of 4-nitorimidazole in the top agarose solution. For large petri dishes $(150 \mathrm{~mm}$ x $25 \mathrm{~mm}) 4$-nitroimidazole diluted in 10 $\mathrm{mL}$ of molten top agarose was used per dish, whereas for small Petri dishes (90 mm x 15 $\mathrm{mm}) 4 \mathrm{~mL}$ of top agarose solution was sufficient. If the cells expressed nitroreductase enzymes from pUCX plasmid, $10 \mu \mathrm{M}$ of IPTG was incorporated into the LB agar prior to plating. An important optimization step throughout this process was the removal of tryptone from the top agarose (included in the original recipe). When tryptone was included in the top agarose cells started to grow within the agarose and began to obscure colonies. After the top agarose had cooled, plates were left at room temperature overnight before being placed at $4{ }^{\circ} \mathrm{C}$ to enhance color development. Colonies expressing a nitroreductase turned a dark purple colour over time. 


\section{Chapter 3: Enhancing the Tinidazole Selectivity of NfsA_Ec using Directed Evolution}

\subsection{Introduction}

\subsubsection{Multiplex Cell Ablation}

While multiple studies had evolved superior nitroreductase enzymes for GDEPT applications (Grove et al., 2003; Guise et al., 2007; Swe et al., 2012), none had optimized them specifically for targeted cell ablation. The goal of this chapter was to address this deficiency by developing nitroreductases suitable for a dual, or "multiplex" cell ablation system. Whereas previous cell ablation studies have used nitroreductase enzymes in combination with a prodrug to ablate single tissues (discussed in Section 1.2.3.2), a multiplex cell ablation system would enable two distinct tissues to be independently ablated within the same model organism. For this to be feasible, two nitroreductase enzymes each possessing non-overlapping nil-bystander prodrug specificities would be required. The first nitroreductase of the pair would selectively activate one prodrug, while the second would selectively activate another, distinct prodrug. Expressing each nitroreductase in a different zebrafish tissue would enable the independent ablation of either tissue in isolation, or, through the administration of both prodrugs, simultaneously (Figure 3.1). Control over the ablation of two tissues would increase the flexibility and scope of cell ablation studies. For example, the same transgenic zebrafish strain could be used to model multiple pathologies, depending on which prodrug was administered and therefore which tissue was ablated. Furthermore, temporal control over the ablation of multiple tissues could assist in studying the role of tissue interaction during developmental and regenerative processes. 


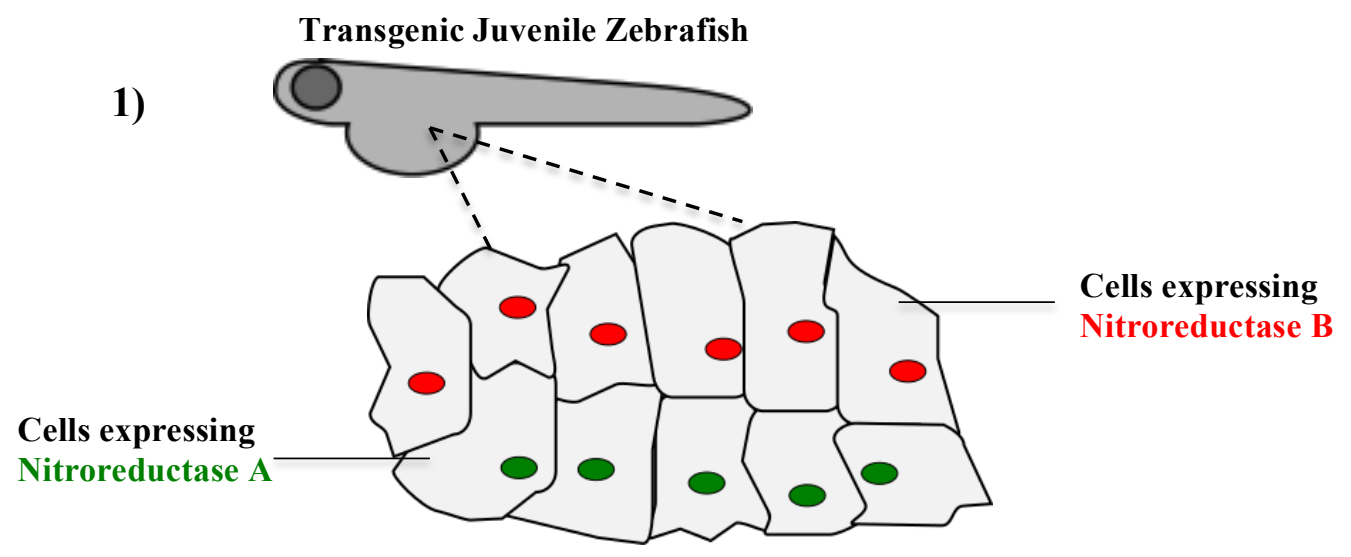

\section{2) Prodrug $A$}

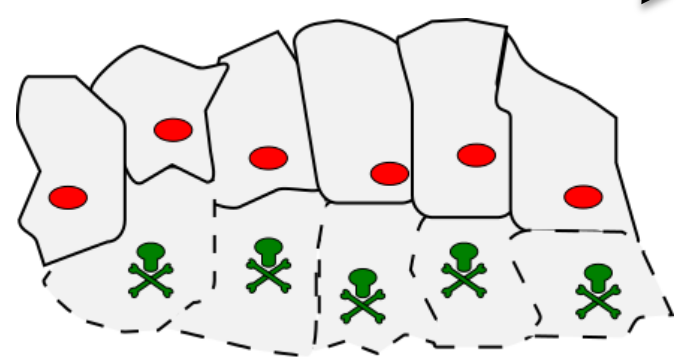

Selective ablation of Nitroreductase A expressing cells
3) Prodrug $B$

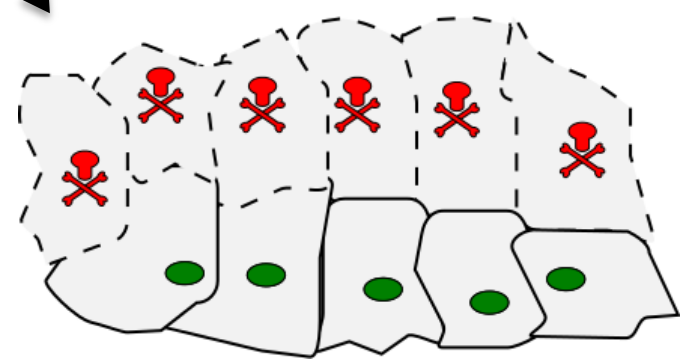

Selective ablation of Nitroreductase B expressing cells

Figure 3.1 - Nitroreductase Multiplex Ablation System. 1) Two distinct nitroreductase enzymes are each expressed in a different tissue in a zebrafish (Danio rerio) using tissue specific promoters. One cell population would only express Nitroreductase A (red nuclei), while the other would only express Nitroreductase B (green nuclei). 2) Nitroreductase A and Nitroreductase B possess non-overlapping nilbystander prodrug specificities, such that the administration of Prodrug A can specifically ablate cells expressing Nitroreductase A, but not cells expressing Nitroreductase B. In contrast, 3) the administration of Prodrug B would specifically ablate cells expressing Nitroreductase B but not Nitroreductase A.

Developing a nitroreductase multiplex ablation system has been one of the ongoing goals in the Ackerley lab. The system has become feasible in recent years as more nitroreductase enzymes and prodrugs, beyond NfsB_Ec and CB1954/metronidazole, have been discovered. The diverse 58-membered core nitroreductase library of the Ackerley lab (Prosser et al., 2013) (discussed in Section 1.3.1) has been particularly useful resource for assessing the relative activities of different nitroreductase enzymes with a given prodrug.

A previous screen of the core 58 nitroreductase library against a panel of 16 different nilbystander prodrugs identified several nitroreductase enzymes possessing a bias towards 
activating certain prodrugs over others (Condon, 2013; Horvat, 2012). Of particular promise for a multiplex ablation system was the finding that the NfsA family members from E. coli (NfsA_Ec) and Pseudomonas putida (NfsA_Pp) (35\% amino acid identity with NfsA_Ec) were, on a molar basis, more effective at sensitising E. coli to the nilbystander prodrug tinidazole than to metronidazole. Like metronidazole, tinidazole is a member of the nil-bystander 5-nitroimidazole family of compounds prescribed to treat both bacterial and parasitic diseases, such as Helicobacter pylori (Berrutti et al., 2008) or Giardia lamblia (Golan et al., 2011) infections, respectively (Figure 3.2). Reduction of the $\mathrm{NO}_{2}$ group of either metronidazole or tinidazole gives rise to a cytotoxic DNA damaging agent (Armstrong and Wilson, 2010). In contrast, NfsB from Vibro vulfinicus $\left(\mathrm{NfsB} \_\mathrm{Vv}\right)$ demonstrated the reverse, possessing on a molar basis greater activity with metronidazole than tinidazole (Condon, 2013; Horvat, 2012). Encouragingly for the use of these enzymes in a eukaryotic cell ablation context, NfsA_Pp and NfsB_Vv maintained their preferential activation of tinidazole and metronidazole, respectively, when expressed from HEK293 cells (Condon, 2013; Horvat, 2012). While other potential nitroreductase enzyme pairs were identified in this initial library screen that exhibited differential specificities for other nil-bystander prodrug candidates, e.g. fluorinated misonidazole and fluorinated etanidazole, these compounds were not as well characterised or readily available as metronidazole and tinidazole (Dr. David Ackerley, personal communication). Metronidazole in particular is favoured by the field due to its relative affordability compared to other cell ablation compounds (White and Mumm, 2013). As the long-term goal of the study was to develop an effective and immediately applicable ablation system, we selected metronidazole and tinidazole as the prodrugs to develop a multiplex ablation system around. 


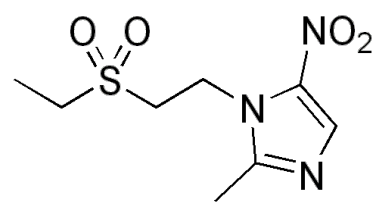

Figure 3.2 - Structure of the 5-nitroimidazole nil-bystander prodrug tinidazole.

Although the combination of NfsA_Ec/NfsA_Pp and $\mathrm{NfsB}$ _Vv was promising, NfsA_Ec/NfsA_Pp still possessed metronidazole activity (Condon, 2013; Horvat, 2012). Likewise, while NfsB_Vv was primarily a metronidazole selective enzyme, it still was also capable of activating tinidazole. It was therefore deemed highly likely that the native NfsA_Ec/NfsA_Pp or NfsB_Vv enzymes might be insufficiently discriminating in their substrate preference to enable effective multiplex ablation. Consideration was therefore given into possible methods of further narrowing the prodrug selectivities of these enzymes. Due to its success in altering nitroreductase activity in the past, a directed evolution approach was chosen.

\subsubsection{Directed Evolution to Evolve Highly Specific Enzymes}

The directed evolution experiments considered in Section 1.4.2 were all performed to improve enzyme activity with particular substrate, the primary focus being enhancement of nitroaromatic prodrug activation by nitroreductase enzymes. However, while less commonly performed, directed evolution can also be used to narrow substrate selectivity, converting enzymes possessing a multiple promiscuous activities into highly specific ones (Nair and Zhao, 2008; Tracewell and Arnold, 2009). There are two general directed evolution strategies for evolving more specific enzymes: either an undesired activity can be negatively selected against, or a desired activity can be continuously selected for (Tracewell and Arnold, 2009). A negative selection pressure can be used to eliminate mutants from a library that possess an undesired activity, often requiring a secondary screen to determine if the desired activity is still present (Tracewell and Arnold, 2009). Negative selection pressures have enabled the evolution of highly selective laccases (Bocola et al., 2004), endopeptidases (Varadarajan et al., 2008a, 2008b), and xylose reductases (Nair and Zhao, 2008). In the case of the xylose reductases, the goal was to eliminate promiscuous L-arabinose reductase activity. There, E. coli cells expressing xylose reductase mutants were plated on LB agar containing L-arabinose, the reduction 
product of which L-arabinitol phosphate, was toxic. The growth of mutants that had retained L-arabinose reductase activity was therefore inhibited (Nair and Zhao, 2008). Rather than selecting against an undesired activity, continuously selecting for a single enzyme function can cause the non-selected functions to weaken and disappear throughout the evolutionary process (Tracewell and Arnold, 2009). Using this strategy has enabled the evolution of enantioselective cyclohexanone monooxygenases (Reetz et al., 2004), lipases (Bocola et al., 2004) and propane monooxygenases (Fasan et al., 2007). Of these two strategies, we chose the former, developing a directed evolution screen that negatively selected against an unwanted prodrug activity.

To narrow the substrate specificity of nitroreductase enzymes, we hypothesised that the conditional toxicity of the prodrugs metronidazole and tinidazole could serve as an effective negative selection pressure. Similar to the xylose reductase evolution discussed above (Nair and Zhao, 2008), we envisaged plating a mutant nitroreductase library in $E$. coli onto LB agar containing metronidazole or tinidazole. Given that nitroaromatic prodrugs only becomes cytotoxic upon reduction by a nitroreductase, we reasoned that only the mutants no longer capable of activating the prodrug should be viable in its presence, forming readily selectable colonies. On the other hand, nitroreductase mutants still capable of converting the prodrug to a cytotoxic form should inhibit their own growth, preventing colony formation.

A consequence of negatively selecting against an enzyme activity can be a general reduction in overall catalytic activity (Nair and Zhao, 2008). Enzymes evolved via this method therefore often also require a positive selection step to improve or retain the desired activity (Tracewell and Arnold, 2009). As metronidazole and tinidazole were both 5-nitroimidazoles, we considered it likely that each substrate would dock in a similar conformation in the active site of a nitroreductase, and that a majority of mutations abrogating activity with one substrate would therefore also impair activity with the other, or indeed lead to a loss of nitroreductase activity in general. To prevent the enrichment of completely inactive nitroreductase mutants it was clear that a positive selection pressure for active nitroreductase enzymes would also be required. The ideal solution would be to utilize positive selection analogues of metronidazole and tinidazole, i.e., compounds that interacted with the nitroreductase active site in an identical manner, but in doing so were detoxified by nitroreduction, rather than being converted to a cytotoxic form. 
Unfortunately, no such compounds are currently known. However, the Ackerley lab recently has discovered that the compound niclosamide can be used as a positive selection agent for generic nitroreductase activity.

\subsubsection{Niclosamide}

The nitroaromatic drug $N$-(2'-chloro-4'-nitrophenyl)-5-chlorosalicylamide, generically called niclosamide (Figure 3.3), was first used clinically in the 1960s to kill parasitic worms via impairment of oxidative phosphorylation (Frayha et al., 1997; Ofori-Adjei et al., 2008).<smiles>O=C(Nc1ccc([N+](=O)[O-])cc1Cl)c1cc(Cl)ccc1O</smiles>

Figure 3.3 - Structure of niclosamide.

Researchers in the Ackerley lab have found that niclosamide is selectively toxic to E. coli cells that lack the efflux transporter TolC (Condon, 2013). However, the same toxicity was not observed when the E. coli cells were overexpressing a nitroreductase (Condon, 2013). Although the exact mechanism was unknown, the protective effect of nitroreductase expression was observed across a range of different families, from the NfsA and NfsB nitroreductases to the AzoR enzymes (Condon, 2013). As nitroreductase activity is required for niclosamide detoxification, we reasoned that including niclosamide in the solid LB agar, along with a 5-nitroimidazole prodrug such as metronidazole, would prevent the enrichment of mutants viable in the presence of metronidazole because they had lost all nitroreductase activity. We reasoned that the generic nitroreductase activity selected through inclusion of niclosamide would translate, in at least some of the mutants, to retention of activity with the other prodrug, tinidazole in this example. If successful, the final product of the evolution would therefore be a mutant nitroreductase that had lost activity with metronidazole while having retained tinidazole activity, resulting in an enhancement of its tinidazole selectivity (Figure 3.4). 
B)

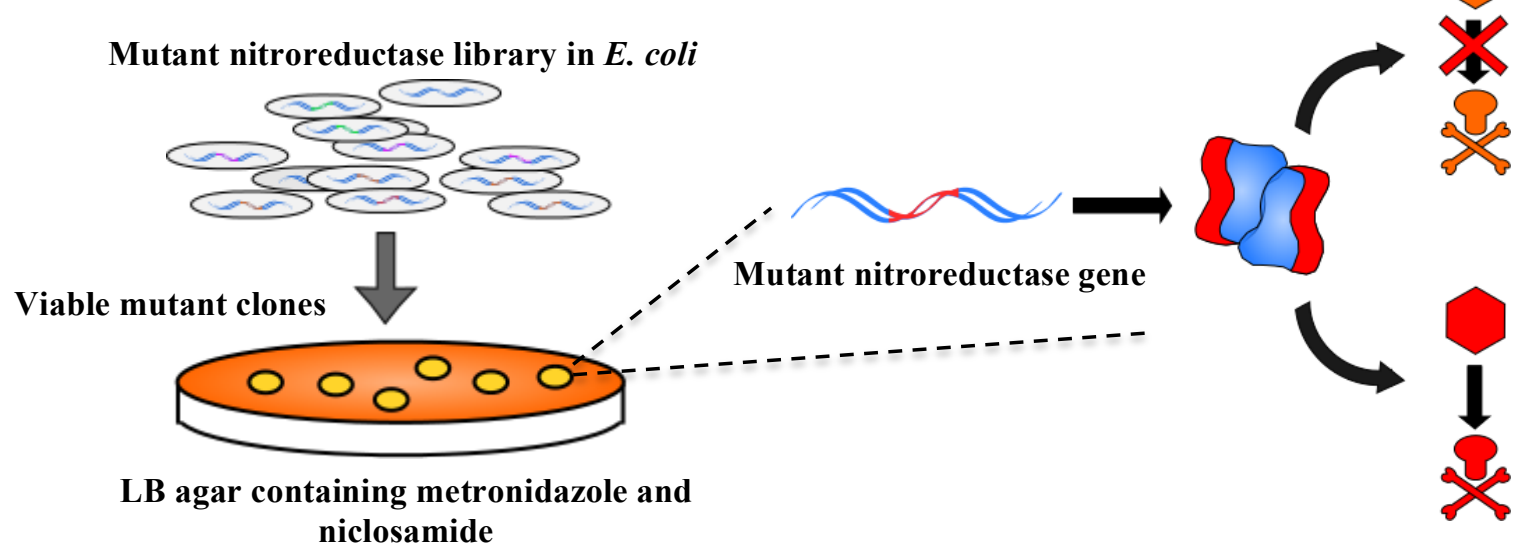

Figure 3.4 - Proposed directed evolution screen for enhancing the tinidazole selectivity of a mutant nitroreductase library expressed from $\boldsymbol{E}$. coli. A) A mutant nitroreductase library expressed from E. coli cells is plated on LB agar supplemented with metronidazole and niclosamide. Metronidazole inhibits the growth of mutants still able to activate it, while niclosamide inhibits the growth of mutants that lack all nitroreductase activity. B) Colonies are then picked and counter-screened to identify mutants that have lost metronidazole activity but retain some level of tinidazole activity.

The system could also potentially be reversed, i.e. supplementing the agar with tinidazole rather than metronidazole, to evolve a complementary mutant enzyme that had lost tinidazole activity but retained metronidazole activity.

\subsubsection{SOS GFP and Growth Inhibition/IC 50 Assays}

In order to determine if the substrate selectivities had changed as a result of the evolution, the metronidazole and tinidazole activities of the recovered nitroreductase mutants would need to be measured. Fortunately, the Ackerley lab has developed multiple methods of rapidly measuring nitroreductase activity with a prodrug substrate. Prominent among these is the use of the E. coli SOS-R4 strain that contains a GFP reporter gene under transcriptional control of an DNA damage responsive (SOS) promoter (Copp et al., 2014). To lower background levels of prodrug activation, the E. coli SOS-R4 strain also contained seven genomic nitroreductase gene deletions (Copp et al., 2014). Transforming a pUCX plasmid containing a candidate nitroreductase gene into the E. coli SOS-R4 strain enables its activity with a nitroaromatic prodrug to be measured. The levels of GFP induction, a product induced by the genotoxic damage caused by an activated prodrug, 
provides a measure of nitroreductase activity with the prodrug (Figure 3.5). Nitroreductases highly active with a prodrug will therefore induce higher levels of GFP expression than those only weakly active.

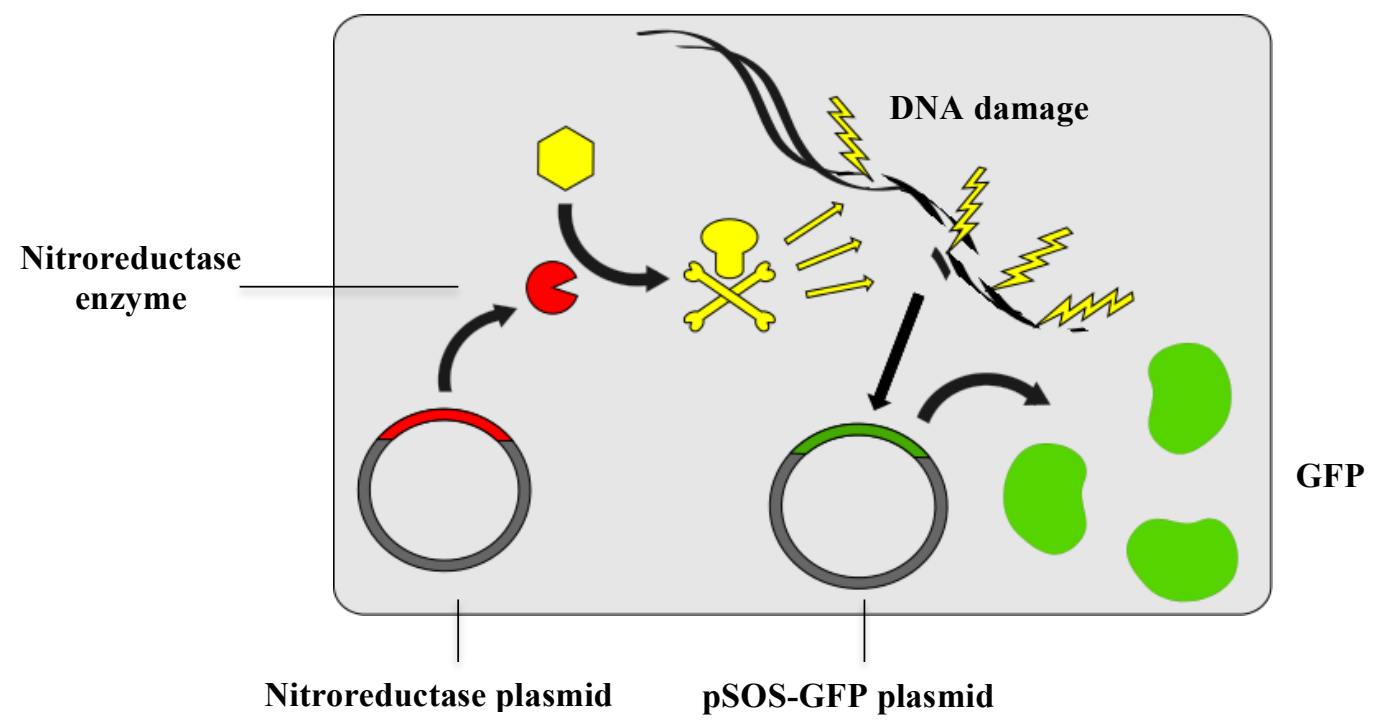

Figure 3.5 - Quantification of prodrug-induced DNA damage using the SOS GFP assay. The E. coli SOS-R4 strain (Copp et al., 2014) has had seven endogenous nitroreductase genes deleted from the chromosome. Two plasmids, each containing a unique antibiotic marker, are present within the E. coli SOS-R4 strain. The first is an expression plasmid containing a candidate nitroreductase gene (red plasmid). The second plasmid contains a copy of the GFP gene under the control of a DNA-damage inducible SOS promoter (pSOS). The DNA damage caused by prodrug activation induces expression of GFP from the SOS promoter. A non-lethal prodrug dose is used to prevent decay of the GFP signal due to cell death. The stronger the GFP signal produced, the more active the nitroreductase is with the prodrug.

Subsequently, as a slower but more definitive method of confirming nitroreductase activity, E. coli SOS-R4 cells overexpressing a candidate nitroreductase gene can be cultured in the presence of a range of concentrations of a prodrug. The extent to which cell growth is inhibited at each concentration relative to an unchallenged control can also provide a measure the nitroreductase activity with the prodrug. From growth inhibition data, an $\mathrm{IC}_{50}$ value can be calculated. $\mathrm{An} \mathrm{IC}_{50}$ value is the concentration of prodrug required to inhibit the growth of a strain by $50 \%$ relative to unchallenged controls. In the case of nitroreductase overexpressing strains, the more effective the nitroreductase is at activating a prodrug, the lower the strain's $\mathrm{IC}_{50}$ value with that prodrug will be. In contrast, $\mathrm{IC}_{50}$ values with prodrugs that are poorer nitroreductase substrates will be larger 
in comparison. Collectively, all of these assays were tool used throughout this thesis for measuring the metronidazole and tinidazole activities of nitroreductase enzymes.

\subsection{Chapter 3 Results}

\subsubsection{Activity of Wild Type Nitroreductase Enzymes with Metronidazole and Tinidazole}

Prior to developing a directed evolution screen to enhance nitroreductase substrate selectivity, the relative activities of wild type NfsA_Pp, NfsB_Vv, and NfsA_Ec with metronidazole and tinidazole were determined using E. coli SOS-R4 growth inhibition assays. The results from the experiments are presented in Figure 3.6 and reflected previous observations (Condon, 2013; Horvat, 2012), where tinidazole was superior than metronidazole at inhibiting the growth of NfsA_Ec and NfsA_Pp overexpressing strains. In contrast, also as previously documented (Condon, 2013; Horvat, 2012), metronidazole was superior to tinidazole at inhibiting the growth of $\mathrm{Nfs} B$ _Vv expressing strains. 
A)

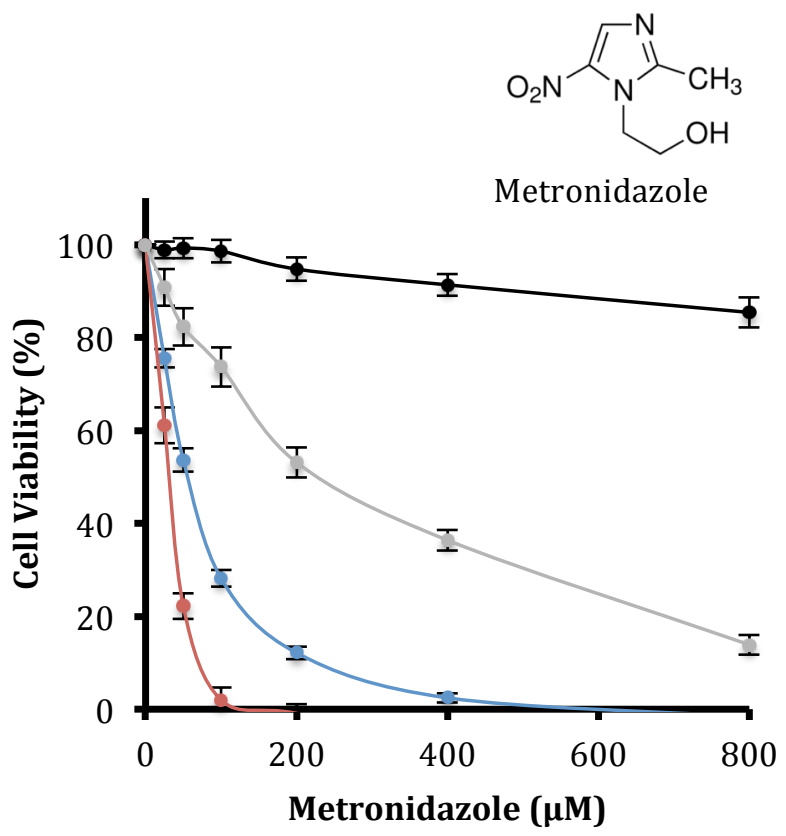

B)

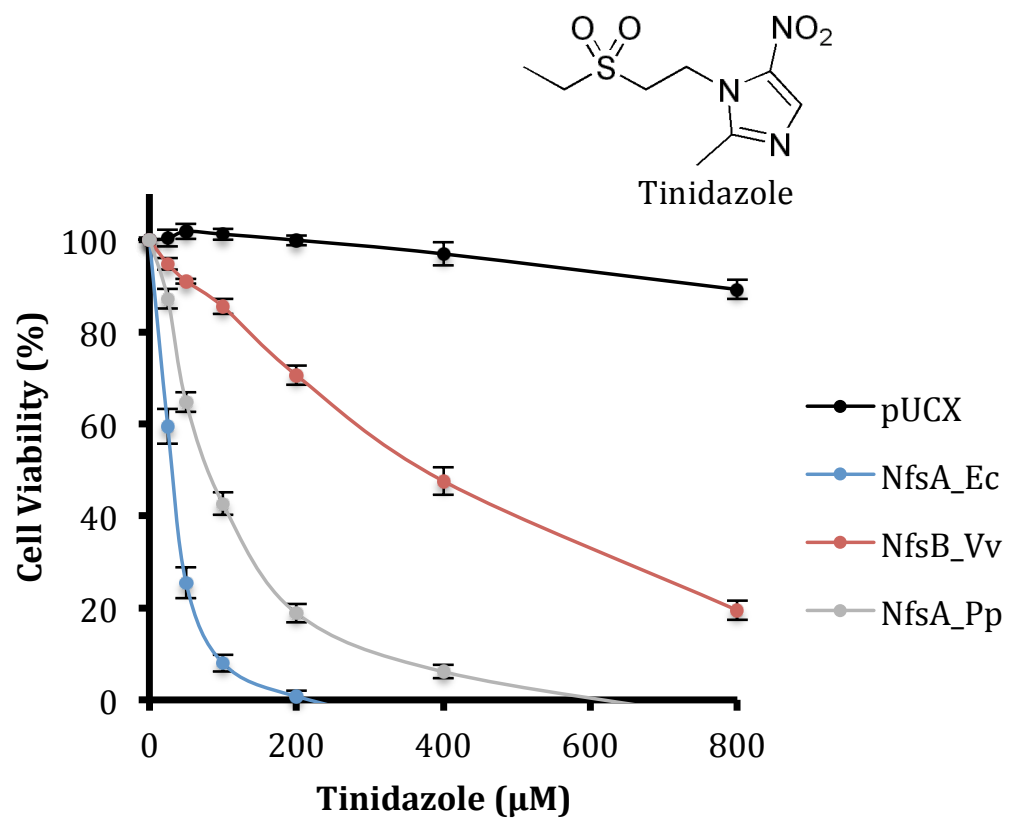

Figure 3.6 - Metronidazole and tinidazole growth inhibition assays of $E$. coli SOS-R4 nitroreductase overexpressing strains. Cultures of E. coli SOS-R4 overexpressing either NfsA_Ec, NfsA_Pp, NfsB_Vv from pUCX plasmids or carrying an empty pUCX plasmid control, were challenged in 384 well plates with serial dilutions of A) Metronidazole or B) Tinidazole for $4 \mathrm{~h}$. Growth was measured as the difference in culture turbidity $\left(\mathrm{OD}_{600}\right)$ between pre $(\mathrm{t}=0 \mathrm{~h})$ and post $(\mathrm{t}=4 \mathrm{~h})$ drug challenged cultures. For each strain, growth inhibition was measured as the percentage decrease between challenged cultures and its respective unchallenged control. Data are representative of five independent experiments \pm SEM for the metronidazole and tinidazole pUCX and $\mathrm{NfsB}_{-} \mathrm{Vv}$ assays. Data are representative of ten independent experiments \pm SEM for the metronidazole NfsA_Ec assay. Data are representative of ten independent experiments \pm SEM for the tinidazole NfsA_Pp assay. Data are representative of seven independent experiments \pm SEM for the metronidazole NfsA_Pp assay.

To provide a measure of substrate selectivity that mutant nitroreductases could be subsequently compared against, $\mathrm{IC}_{50}$ values were calculated from the growth inhibition data of each wild type enzyme from Figure 3.6. Metronidazole and tinidazole $\mathrm{IC}_{50}$ values for $E$. coli SOS-R4 cells overexpressing NfsA_Ec, NfsA_Pp, and NfsB_Vv are presented in Table 3.1. 


\begin{tabular}{|c|c|c|c|}
\hline Enzyme & Tinidazole $I C_{50}(\mu M)$ & Metronidazole $I C_{50}(\mu M)$ & $\begin{array}{l}\text { Tinidazole Selectivity (Metronidazole } I C_{50} \\
\text { Tinidazole } I C_{50} \text { ) }\end{array}$ \\
\hline NfsA_Ec & $30 \pm 2$ & $55 \pm 3$ & 2 \\
\hline \multirow[t]{2}{*}{ NfsA_Pp } & $80 \pm 6$ & $240 \pm 23$ & 3 \\
\hline & & & $\begin{array}{l}\text { Metronidazole Selectivity (Tinidazole } I C_{50} \\
\text { Metronidazole } I C_{50}\end{array}$ \\
\hline NfsB_Vv & $380 \pm 29$ & $29 \pm 2$ & 13 \\
\hline
\end{tabular}

Table 3.1 - Wild Type Nitroreductase Metronidazole and Tinidazole IC $_{50}$ Values. GraphPad Prism $6^{\mathrm{TM}}$ was used to calculate metronidazole and tinidazole $\mathrm{IC}_{50}$ values for NfsA_Ec, NfsA_Pp and NfsB_Vv using non-linear regression analysis from the growth inhibition data of Figure 3.6. Fold difference between $\mathrm{IC}_{50}$ values was determined from the quotient of the two values. Data are average of at least five independent experiments \pm SEM.

The results demonstrated that on a molar basis, NfsA_Pp had a 3 fold lower $\mathrm{IC}_{50}$ value for tinidazole relative to metronidazole, while the same ratio for NfsA_Ec was only two fold lower (Table 3.1). In contrast, on a molar basis, $\mathrm{NfsB}_{-} \mathrm{Vv}$ was far more metronidazole selective, possessing a 13 fold lower $\mathrm{IC}_{50}$ with metronidazole than with tinidazole. For a multiplex ablation system to be possible, however, two highly selective nitroreductase enzymes are required. Given the larger initial substrate selectivity demonstrated by NfsB_Vv (Tinidazole $\mathrm{IC}_{50}=380 \pm 29 \mu \mathrm{M}$, Metronidazole $\mathrm{IC}_{50}=29 \pm 2$ ), the NfsA family enzymes were targeted first targeted to improve their tinidazole selectivity using directed evolution. NfsA_Ec and NfsA_Pp were both evolved in parallel to be more discriminating against metronidazole. While NfsA_Ec was not as intrinsically as tinidazole selective as NfsA_Pp, a high diversity site-saturation mutant library (NfsA_Ec 7SM) had previously been generated for another ongoing project within the Ackerley lab, and was available to this research, providing a validated high quality mutant library as an excellent starting point for directed evolution.

\subsubsection{Positive Selection of Active NfsA_Ec 7SM Mutants using Niclosamide}

Dr. Janine Copp, Dr. Elsie Williams, and Dr. David Ackerley (unpublished) created the NfsA_Ec 7SM library in a semi-rational manner by targeting seven codons corresponding to amino acid residues within the active site of the enzyme. Each of the sites was replaced with an NDT codon (with the exception of codon 83, which was targeted with an 
NNK codon), allowing twelve different amino acids, covering all major functional groups (i.e., acidic, basic, small hydrophobic, etc), to be represented at the position. Five of the sites were selected empirically based on previous observations that amino acid changes at those sites had increased enzyme activity with the prodrug PR-104A and/or the PET imaging agent EF5. The remaining two sites were identified using the bioinformatics tool HotSpot Wizard (www.loschmidt.chemi.muni.cz/hotspotwizard/) based on the location and evolutionary conservation of each site. Targeting these seven residues produced a possible 95 million different NfsA_Ec variants, all cloned into pUCX plasmids. Before any library screening could commence, however, the directed evolution screen was optimized.

An important premise of the proposed directed evolution screen was that niclosamide would be capable of imposing a positive selection for active nitroreductase mutants while inhibiting the growth of those that had lost all nitroreductase activity. Given the high active site mutation rate of the NfsA_Ec 7SM library, we predicted the majority of mutants would no longer be catalytically functional. Previous empirical testing by the Ackerley lab had determined that LB agar containing $0.5 \mu \mathrm{M}$ niclosamide was capable of inhibiting the growth of $E$. coli SOS-R4 cells lacking nitroreductase expression (containing an empty pUCX plasmid), but not overexpressing NfsA_Ec (Dr. Elsie Williams, Dr Janine Copp, Unpublished). The same selection for E. coli SOS-R4 cells expressing NfsA_Ec was observed when LB agar containing $0.5 \mu \mathrm{M}$ niclosamide was tested in this thesis (Figure 3.7). 

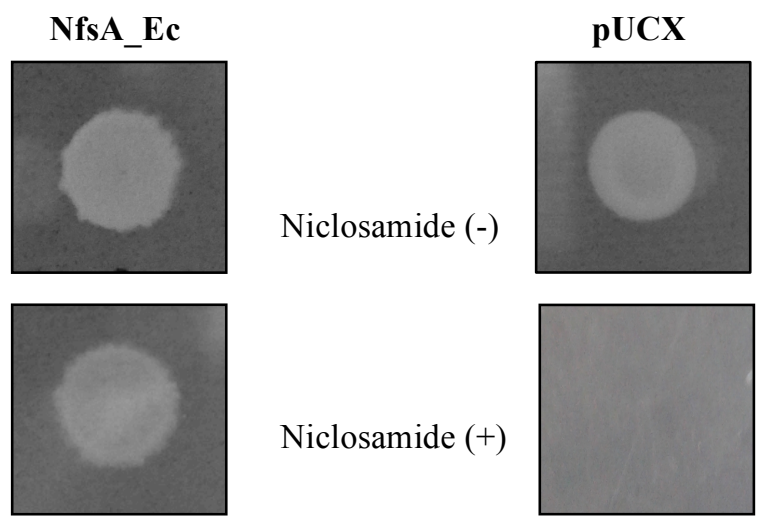

Figure 3.7 - Viability of $E$. coli SOS-R4 cells overexpressing NfsA_Ec on niclosamide. Midexponential phase E. coli SOS-R4 cultures expressing NfsA_Ec from pUCX or containing an empty pUCX plasmid were replica plated on either unsupplemented LB agar niclosamide (-) or LB agar containing 0.5 $\mu \mathrm{M}$ niclosamide $(+)$. The LB agar plates were then incubated overnight to promote cell growth on the solid media. These replica plating results were equivalent to those observed at an individual colony level when the day cultures were each spread on LB agar plates containing the same compound concentrations.

However, as niclosamide possessed quite a different structure to metronidazole and tinidazole, the effects of niclosamide selection on activities with these prodrugs within a mutant nitroreductase library were unknown. In particular, we wanted to determine that selecting nitroreductase mutants on the basis of viability on niclosamide was not detrimental to tinidazole activity. To test this, E. coli SOS-R4 cells expressing random NfsA_Ec 7SM library plasmids were plated on either unamended LB agar or LB agar containing $0.5 \mu \mathrm{M}$ niclosamide. A random selection of colonies, 57 from the $0.5 \mu \mathrm{M}$ niclosamide LB agar plate and 57 from the control plate, were then picked and grown as individual liquid cultures. Tinidazole and metronidazole SOS assays were then performed on each NfsA_Ec 7SM mutant liquid culture to determine their activity with each prodrug (Figure 3.8A and Figure 3.8B). The results demonstrated that all of the NfsA_Ec 7SM mutants collected from LB agar plates containing $0.5 \mu \mathrm{M}$ niclosamide were active with both metronidazole and tinidazole, with the majority of mutants even demonstrated higher levels of activity than wild type NfsA_Ec. 

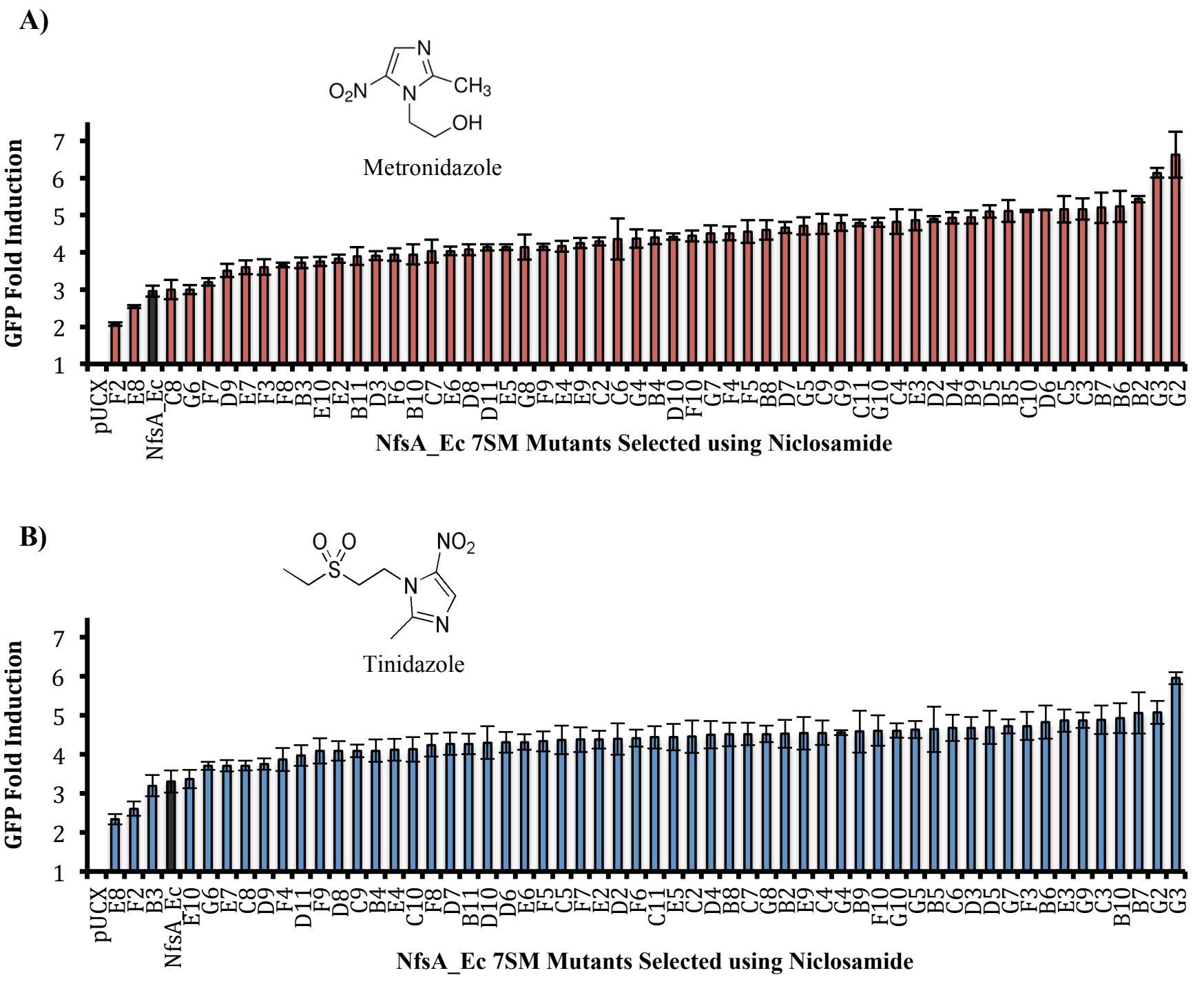

Figure 3.8 - Metronidazole and tinidazole induced GFP SOS response in $E$. coli SOS-R4 overexpressing NfsA_Ec 7SM mutants preselected on niclosamide. A total of 57 NfsA_Ec 7SM mutant colonies were randomly selected from LB agar containing $0.5 \mu \mathrm{M}$ niclosamide and challenged using either metronidazole or tinidazole in replicate GFP SOS assays. Mid-exponential cultures from each of the 57 colonies were challenged in 384 well plates with either A) $5 \mu \mathrm{M}$ metronidazole or B) $5 \mu \mathrm{M}$ tinidazole for 3 hours, after which GFP fluorescence $(488 \mathrm{ex} / 510 \mathrm{em})$ and culture turbidity $\left(\mathrm{OD}_{600}\right)$ were recorded. A DMSO control (unchallenged) was also included for each of the 57 7SM mutants. The raw GFP value of each culture was normalized by its $\mathrm{OD}_{600}$. GFP fold induction was then calculated from the difference between the normalized GFP values of each challenged culture, and its respective unchallenged control. $E$. coli SOS-R4 cells expressing wild type pUCX NfsA_Ec is highlighted in black. pUCX represents E. coli SOS-R4 cells containing an empty pUCX plasmid. Data are representative of three independent experiments \pm SEM. 


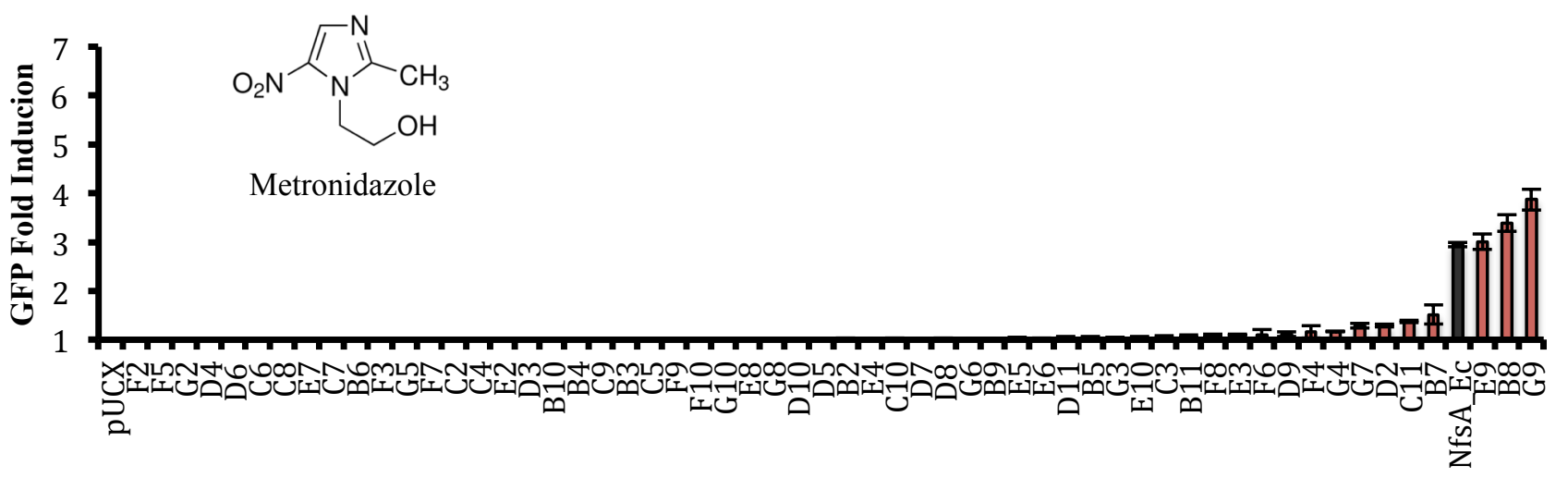

NfsA_Ec 7SM Mutants Lacking Niclosamide Selection

B)

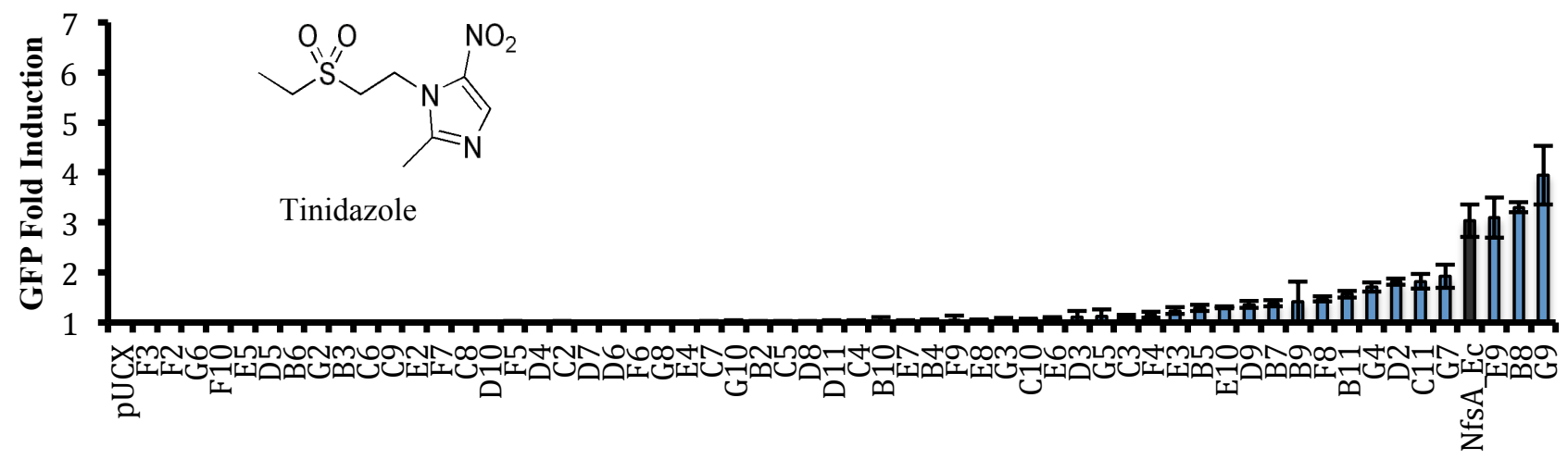

NfsA_Ec 7SM Mutants Lacking Niclosamide Selection

Figure 3.9 - GFP SOS response induced by metronidazole or tinidazole in randomly selected $E$. coli SOS-R4 overexpressing NfsA_Ec 7SM mutants in the absence of niclosamide preselection. A total of 57 NfsA_Ec 7SM mutant colonies were randomly selected from unsupplemented LB agar and challenged using metronidazole and tinidazole in GFP SOS assays. Mid-exponential cultures from each of the 57 colonies were challenged in 384 well plates with either A) $5 \mu \mathrm{M}$ metronidazole or B) $5 \mu \mathrm{M}$ tinidazole for 3 hours, after which GFP fluorescence $(488 \mathrm{ex} / 510 \mathrm{em})$ and culture turbidity $\left(\mathrm{OD}_{600}\right)$ were recorded. A DMSO control (unchallenged) was also included for each of the 57 7SM mutants. The raw GFP value of each culture was normalized by its $\mathrm{OD}_{600}$. GFP fold induction was then calculated from the difference between the normalized GFP values of each challenged culture, and its respective unchallenged control. $E$. coli SOS-R4 cells expressing wild type pUCX NfsA_Ec is highlighted in black. pUCX represents E. coli SOS-R4 cells containing an empty pUCX plasmid. Data are representative of three independent experiments \pm SEM.

To confirm the activity changes observed in the SOS assays of Figure 3.8 growth inhibition assays using $50 \mu \mathrm{M}$ of either prodrug were performed on the same sets of 57 
NfsA_Ec 7SM mutants. The results, (presented in Appendix Figure 8.1) were consistent with the SOS data, indicating that the majority of NfsA_Ec 7SM mutants selected using niclosamide were more active with metronidazole and tinidazole than wild type NfsA_Ec. Also consistent with the SOS data in Figure 3.9, the growth of NfsA_Ec 7SM mutants lacking $0.5 \mu \mathrm{M}$ niclosamide were generally uninhibited $50 \mu \mathrm{M}$ metronidazole or tinidazole, indicating a loss of activity with these prodrugs (Appendix Figure 8.2).

Collectively, the results demonstrated that niclosamide selection was capable of enriching for nitroreductase mutants active with both metronidazole and tinidazole. As no obvious bias against either of the prodrugs was observed (the retention of tinidazole activity being particularly important for the evolution of tinidazole selective NfsA_Ec mutants) we concluded niclosamide could be used as a positive selection agent for nitroreductase enzymes in the directed evolution screen. The next component of the directed evolution screen that required optimization was the concentration of metronidazole needed to select against NfsA_Ec 7SM mutants possessing metronidazole activity.

\subsubsection{Optimization of Metronidazole Concentrations for Negative Selection}

To select against NfsA_Ec mutants possessing substantially less metronidazole activity than wild type NfsA_Ec, it was necessary that the metronidazole concentration used inhibit NfsA_Ec 7SM mutant clones possessing similar levels of metronidazole activity. During these experiments the azoreductase enzyme from E. coli (AzoR_Ec) provided a valuable control. Azoreductases are FMN-dependent enzymes able to catalyze the cleavage of azo groups $(-\mathrm{N}=\mathrm{N}-)$ (Nakanishi et al., 2001). Additionally, some AzoR enzymes have also demonstrated nitroreductase activity (Prosser et al., 2013, 2010). Here, AzoR_Ec was useful because previous testing had indicated that it lacked activity with metronidazole, but was still able to confer protection against niclosamide (Condon, 2013). We reasoned that AzoR_Ec could therefore be used as a surrogate for an NfsA_Ec 7SM mutant that had lost metronidazole activity but not all nitroreductase activity (i.e. the target of the evolution).

To confirm this previous observation, niclosamide, metronidazole, and tinidazole growth inhibition assays were performed using E. coli SOS-R4 expressing either AzoR_Ec or 
NfsA_Ec from a pUCX plasmid, or containing an empty pUCX plasmid (Figure 3.10). These experiments confirmed E. coli SOS-R4 expressing AzoR_Ec from pUCX cells were essentially as resistant to metronidazole and tinidazole as the empty plasmid control strain (metronidazole and tinidazole $\mathrm{IC}_{50}$ values $>800 \mu \mathrm{M}$ ) (Figure 3.10B and Figure 3.10C). However, similar to NfsA_Ec, AzoR_Ec expressing clones remained viable in the presence of niclosamide (Figure 3.10A) indicating they still possessed nitroreductase activity. As also observed in Figure 3.7, E. coli SOS-R4 clones lacking functional nitroreductase expression (containing an empty pUCX plasmid) were strongly inhibited by low levels (160 nM) of niclosamide (Figure 3.10A). 
A)

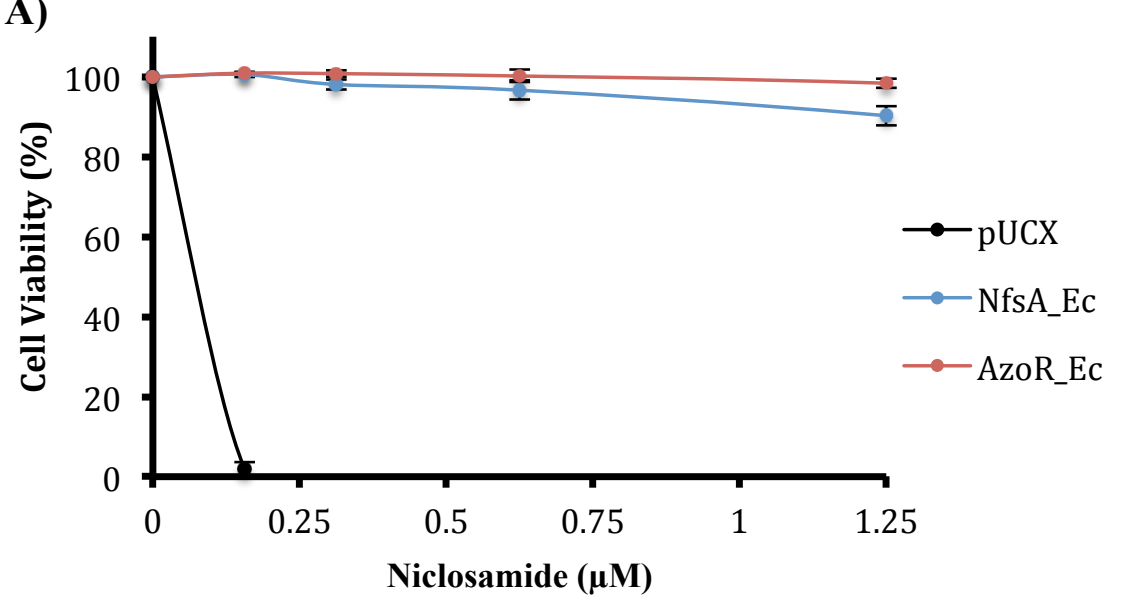<smiles>O=C(Nc1ccc([N+](=O)[O-])cc1Cl)c1cc(Cl)ccc1O</smiles>

Niclosamide

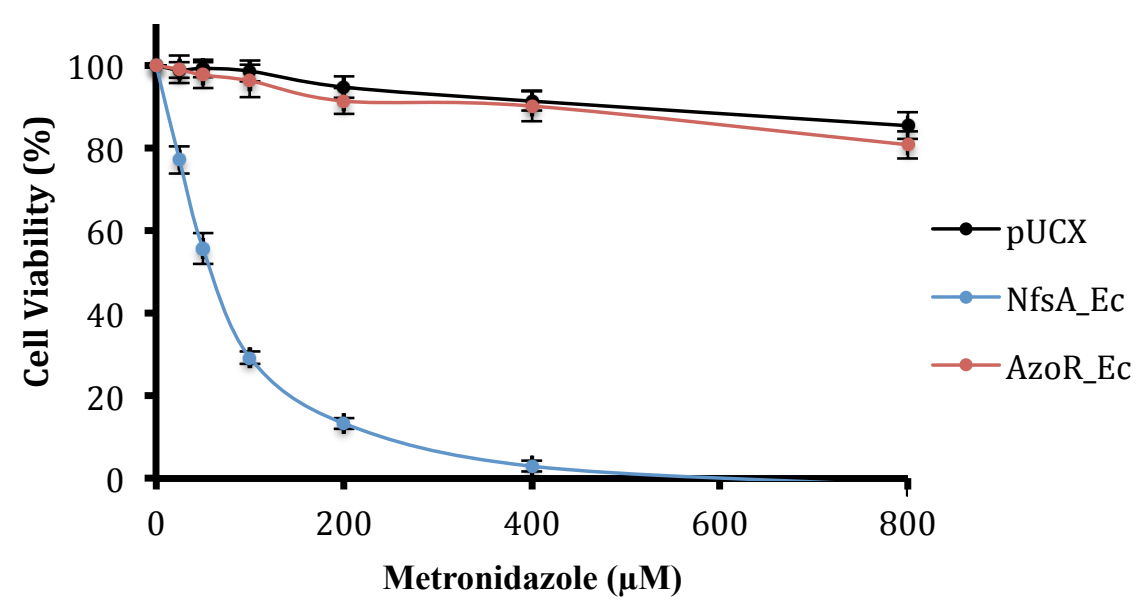

C)

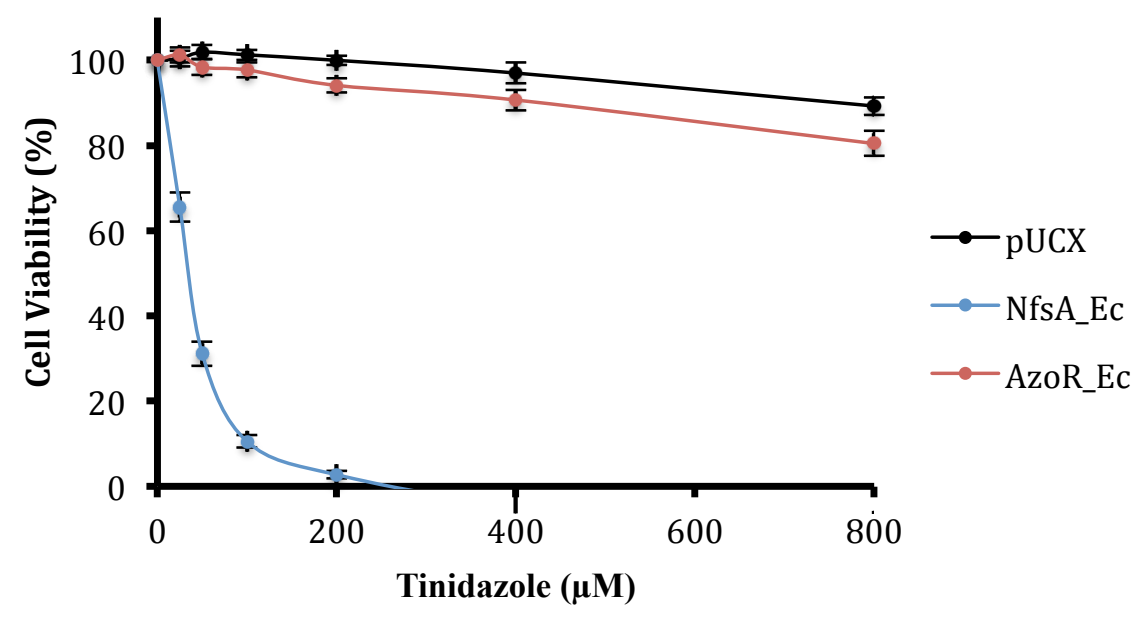<smiles>Cc1ncc([N+](=O)[O-])n1CCO</smiles>

Metronidazole

Figure 3.10 - Niclosamide, metronidazole and tinidazole growth inhibition assays of $E$. coli SOS-R4 nitroreductase overexpressing strains. Mid-exponential E. coli SOS-R4 cultures expressing either NfsA_Ec or AzoR_Ec from pUCX, or containing an empty pUCX plasmid were challenged in 384 well plates with serial dilutions of A) Niclosamide, B) Metronidazole or C) Tinidazole for $4 \mathrm{~h}$. Growth was measured as the difference in culture turbidity $\left(\mathrm{OD}_{600}\right)$ between pre $(\mathrm{t}=0 \mathrm{~h})$ and post $(\mathrm{t}=4 \mathrm{~h})$ drug challenged cultures. For each strain, growth inhibition was measured as the percentage decrease in growth between each challenged culture and its respective unchallenged control. Data are representative of three independent experiments \pm SEM for the niclosamide growth inhibition assays. Data are representative of five independent experiments \pm SEM for the metronidazole and tinidazole growth inhibition assays. 
From these results, we reasoned that finding metronidazole and niclosamide LB agar concentrations that inhibited the growth of E. coli SOS-R4 NfsA_Ec and pUCX clones, but not AzoR_Ec clones, would identify appropriate concentrations for screening the NfsA_Ec 7SM library. In combination with the validated $0.5 \mu \mathrm{M}$ niclosamide concentration (Figure 3.7), a range of different metronidazole concentrations was tested to identify such a concentration. For each condition tested, protein expression was induced in day cultures of E. coli SOS-R4 cells expressing AzoR_Ec or NfsA_Ec from pUCX, or containing an pUCX empty plasmid. Each day culture was then plated on a separate agar plate containing $0.5 \mu \mathrm{M}$ niclosamide and a metronidazole concentration from $0.2 \mathrm{mM}$ to $1 \mathrm{mM}$. LB agar containing $1 \mathrm{mM}$ metronidazole and $0.5 \mu \mathrm{M}$ niclosamide was found to inhibit the growth of the NfsA_Ec and pUCX control strains, whereas SOS-R4 cells expressing AzoR_Ec remained viable (Figure 3.11).

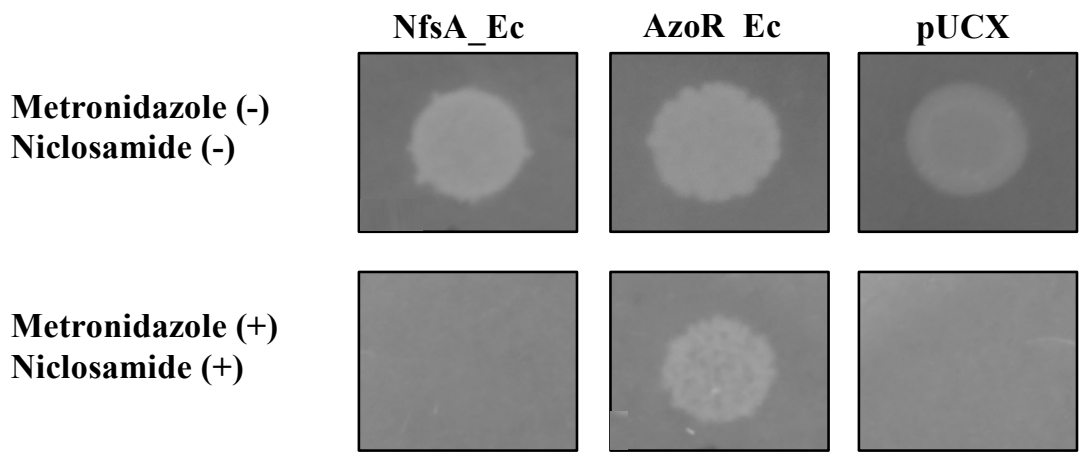

Figure 3.11 - Replica plating of $E$. coli SOS-R4 nitroreductase overexpressing strains on metronidazole and tinidazole. Mid-exponential phase nitroreductase E. coli SOS-R4 expressing AzoR_Ec or NfsA_Ec from pUCX, or containing an pUCX empty plasmid were replica plated either on unsupplemented LB agar (metronidazole (-), niclosamide (-)) or LB agar containing $1 \mathrm{mM}$ metronidazole and $0.5 \mu \mathrm{M}$ niclosamide (metronidazole $(+)$, niclosamide $(+)$ ). The LB agar plates were then incubated overnight to promote growth on the solid media. These replica plating results were equivalent to those observed at an individual colony level when the day cultures were each spread on LB agar plates containing the same compound concentrations. Colonies of E. coli SOS-R4 pUCX were viable on LB agar containing $1 \mathrm{mM}$ metronidazole alone, indicating niclosamide was responsible for their lack of viability (not shown).

Before library screening was performed, a mock directed evolution experiment was performed to further validate the preferred $(1 \mathrm{mM})$ metronidazole and niclosamide $(0.5$ $\mu \mathrm{M})$ concentrations. 


\subsubsection{Preliminary Testing of Directed Evolution Parameters}

A highly diverse collection of mutants such as the 7SM NfsA_Ec library would likely contain nitroreductase mutants possessing a range of activities, from mutants that were no longer functional nitroreductase (highly likely), to mutants possessing wild type levels of activity, and - our initial target - mutants possessing decreased levels of metronidazole activity still able to detoxify niclosamide. The capacity for enrichment of small initial concentrations of the latter clones was tested by creating a mixed culture of $E$. coli SOSR4 pUCX $n f_{S} A_{-} E c$, pUCX empty, and pUCX azoR_Ec cells, in doing so imitating the range of activities predicted to be present in the NfsA_Ec 7SM library. In this context, as discussed in Section 3.2.3, AzoR_Ec could serve as a surrogate for active nitroreductase mutants that had lost activity with metronidazole. Similarly, pUCX could be a surrogate for a nitroreductase mutant possessing no function nitroreductase activity. NfsA_Ec, on the other hand, could represent NfsA_Ec 7SM mutants possessing wild type levels of metronidazole activity. To test the AzoR_Ec enrichment capacity of our directed evolution screen, a mixed culture of $E$. coli SOS-R4 cells was established comprising $49.9 \%$ pUCX clones, $49.9 \%$ pUCX $n f_{S} A_{-} E c$, and $0.2 \%$ pUCX azoR_Ec cells. This cell mixture was then plated on LB agar containing $1 \mathrm{mM}$ metronidazole and $0.5 \mu \mathrm{M}$ niclosamide. Twenty-four of the colonies that subsequently formed were randomly screened using PCR to determine whether they contained an $n f S A \_E c, a z o R \_E c$, or empty pUCX plasmid. The results from the PCR screening revealed that upon plating on metronidazole and niclosamide, $100 \%$ of colonies screened contained AzoR_Ec plasmids (Figure 3.12). 


\section{Colonies picked from $1 \mathrm{mM}$ metronidazole and $0.5 \mu \mathrm{M}$ niclosamide supplemented LB agar}

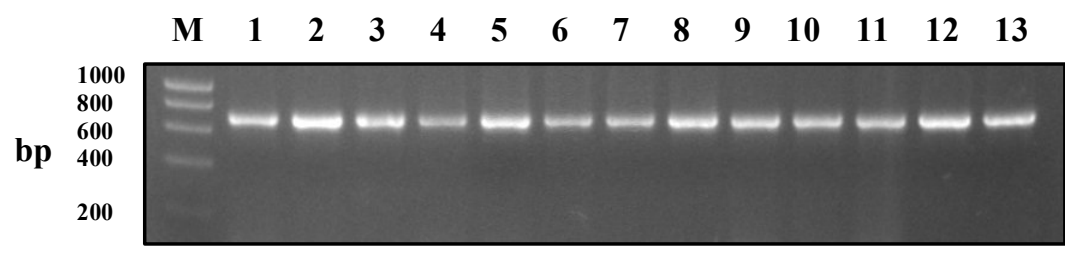

$\begin{array}{llllllllllllll}M & 14 & 15 & 16 & 17 & 18 & 19 & 20 & 21 & 22 & 23 & 24 & (+) & (-)\end{array}$

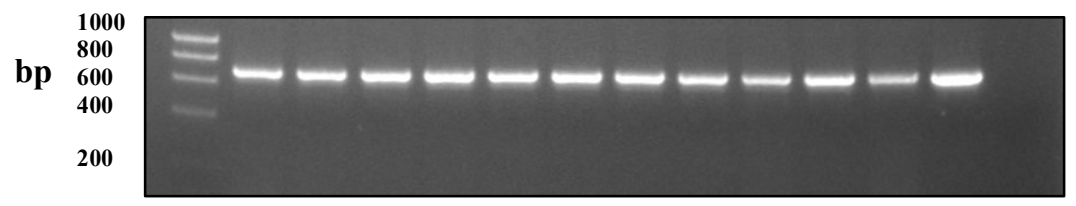

Figure 3.12 - PCR screening of individual colonies from a mixed culture of $E$. coli SOS-R4 pUCX empty (49.9\%), NfsA_Ec (49.9\%) and AzoR_Ec (0.2\%) cells plated on LB agar supplemented with metronidazole and niclosamide. E. coli SOS-R4 cells containing azoR_Ec, $n f_{S} A_{-} E c$, or empty pUCX plasmids were combined (after $\mathrm{OD}_{600}$ values had been normalized) in a mixed culture where pUCX $n f S A \_c$ and pUCX empty cells each made up $49.9 \%$ and pUCX azoR_Ec cells comprised $0.2 \%$ of the total culture. The mixture was plated on LB agar supplemented with $1 \mathrm{mM}$ metronidazole and $0.5 \mu \mathrm{M}$ niclosamide. After colony formation, 24 colonies were screened at random using a pUCX specific forward primer and an azoR_Ec specific reverse primer. PCR products were run on a $1 \%(\mathrm{w} / \mathrm{v})$ agarose gel stained with ethidium bromide to identify PCR amplification $\left(a z o R_{-} E c=606 \mathrm{bp}\right) . \mathrm{M}=\mathrm{DNA}$ size standards. $(+)=$ PCR from purified pUCX AzoR_Ec plasmid. (-) = Template free negative control. The same 24 colonies were also counter-screened with $n f_{S} A_{-} E c$ and pUCX empty specific primers, confirming they did not contain either of these plasmids (not shown).

In contrast, no AzoR_Ec enrichment was observed when the same E. coli SOS-R4 culture mixture was plated on LB agar lacking both metronidazole and niclosamide (Figure 3.13). 
Colonies picked from unsupplemented LB agar

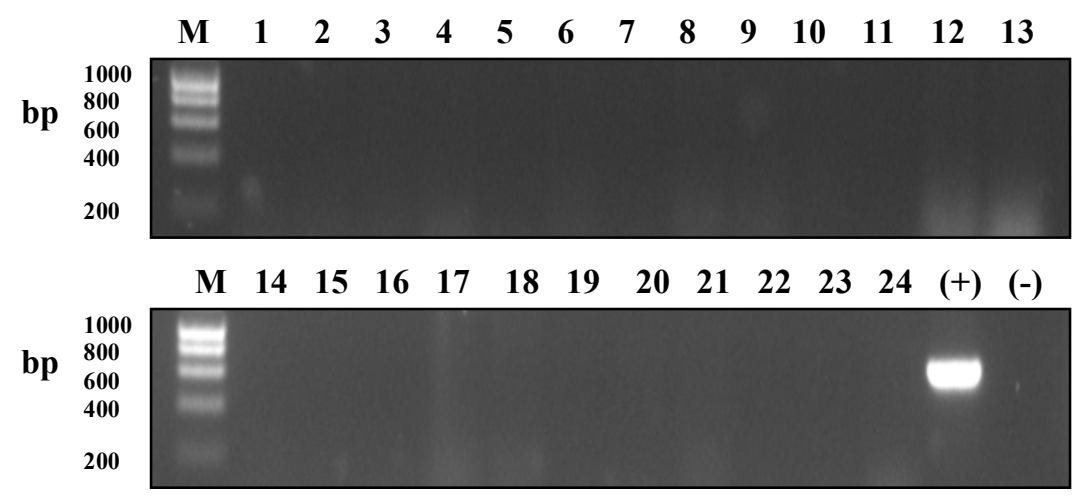

Figure 3.13 - PCR screening of individual colonies from a mixed culture of $E$. coli SOS-R4 pUCX_Empty (49.9\%), NfsA_Ec (49.9\%) and AzoR_Ec (0.2\%) cells plated on unsupplemented LB agar. E. coli SOS-R4 cells containing $a z o R_{-} E c, n f s A_{-} E c$, or empty pUCX plasmids were combined (after $\mathrm{OD}_{600}$ values had been normalized) in a mixed culture where pUCX $n f S A \_E c$ and pUCX empty cells each made up $49.9 \%$ and pUCX azoR_Ec cells comprised $0.2 \%$ of the total culture. The mixture was plated on LB agar lacking metronidazole and niclosamide. After colony formation, 24 colonies were screened at random using a pUCX specific forward primer and an $a z o R_{-} E c$ specific reverse primer. PCR products were run on a $1 \%(\mathrm{w} / \mathrm{v})$ agarose gel stained with ethidium bromide to identify PCR amplification (azoR_Ec $=606 \mathrm{bp}) . \mathrm{M}=$ DNA size standards. $(+)=$ PCR from purified pUCX AzoR_Ec plasmid. $(-)=$ Template free negative control. Subsequent PCR screening of these colonies revealed the majority of colonies contained pUCX empty plasmids.

These results demonstrated that LB agar supplemented with $1 \mathrm{mM}$ metronidazole and 0.5 $\mu \mathrm{M}$ niclosamide was capable of enriching for E. coli SOS-R4 cells expressing AzoR_Ec, even when these cells made up only a small fraction of the total population. Collectively, these results suggested that the directed evolution screen should enrich for any NfsA_Ec 7SM mutants lacking metronidazole activity, but retaining the ability to detoxify niclosamide, present in the NfsA_Ec 7SM library. With the evolution parameters optimized and validated library screening could now commence.

\subsubsection{NfsA_Ec 7SM Library Screening using Metronidazole and Niclosamide Supplemented LB agar}

The majority of library screening was performed using the $1 \mathrm{mM}$ metronidazole concentration validated in Section 3.2.3 and Section 3.2.4. However, the metronidazole concentration of $800 \mu \mathrm{M}$ was also tested for several of the library plates, as it was also 
found to inhibit the growth of E. coli SOS-R4 cells expressing NfsA_Ec. Both concentrations were tested in case the higher metronidazole concentration $(1 \mathrm{mM})$ selected too strongly against metronidazole activity, modifying the active site such that all 5-nitroimidazole activity was lost. However, no noticeable difference in outcome between the two concentrations was observed in this work.

E. coli SOS-R4 cells containing the NfsA_Ec 7SM library were plated on LB agar containing $0.5 \mu \mathrm{M}$ niclosamide and $1 \mathrm{mM}$ metronidazole. NfsA_Ec 7SM Mutants that formed colonies on the LB agar were picked and grown as liquid cultures. SOS GFP assays were then performed to determine their relative levels of metronidazole and tinidazole activity post metronidazole and niclosamide selection. A total of 500 colonies picked from metronidazole and niclosamide supplemented LB agar plates were screened in this manner. An example of metronidazole and tinidazole SOS GFP assays run on a subset (55) of the selected NfsA_Ec 7SM colonies is displayed in Figure 3.14. 


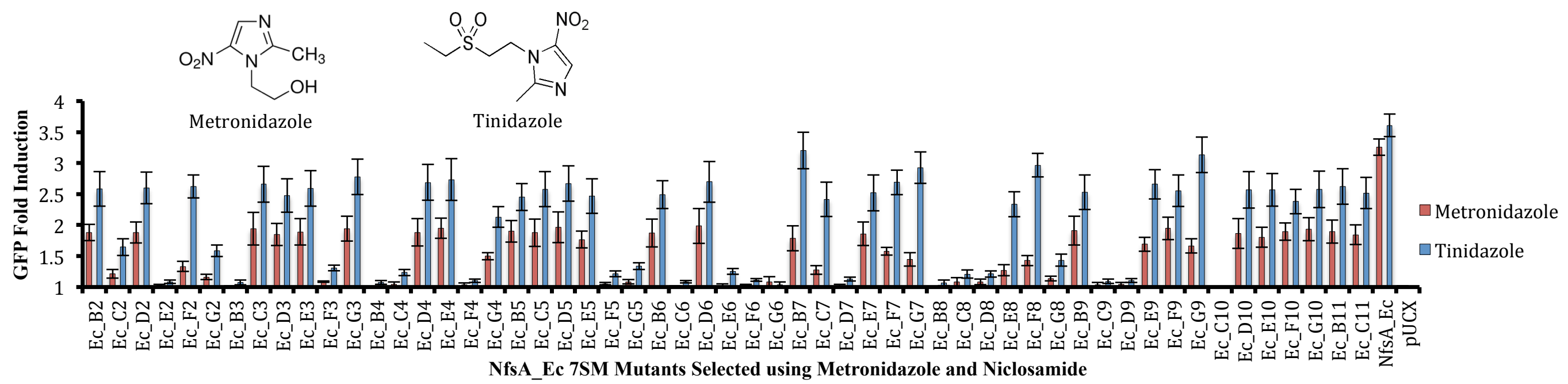

Figure 3.14 - Metronidazole and tinidazole induced SOS GFP response in $E$. coli SOS-R4 overexpressing niclosamide and metronidazole preselected nitroreductase clones. Mid-exponential cultures of $55 \mathrm{Nfs} A$ Ec $7 \mathrm{SM}$ mutants selected on LB agar containing $800 \mu \mathrm{M}$ metronidazole and $0.5 \mu \mathrm{M}$ niclosamide were challenged in 384 well plates containing either $5 \mu \mathrm{M}$ metronidazole or $5 \mu \mathrm{M}$ tinidazole for 3 hours, after which GFP fluorescence (488 ex/510 em) and culture turbidity $\left(\mathrm{OD}_{600}\right)$ were recorded. A DMSO control (unchallenged) was also included for each strain. The raw GFP values of each challenged culture and their respective unchallenged control were normalized by $\mathrm{OD}_{600}$. GFP fold induction was then calculated from the difference between the normalized GFP values of each challenged culture and its respective unchallenged control. E. coli SOS-R4 clones expressing the wild type NfsA_Ec from pUCX was included for the purposes of comparison. pUCX represents E. coli SOS-R4 cells containing an empty pUCX plasmid. Data are representative of three \pm SEM independent experiments for the metronidazole SOS assay using cultures of the same NfsA_Ec 7SM mutants. Data are representative of six independent experiments \pm SEM for tinidazole using cultures of the same NfsA_Ec 7SM mutants. 
All of the NfsA_Ec 7SM colonies screened in Figure 3.14 had lower metronidazole SOS responses than wild type NfsA_Ec. While some mutants had also lost all tinidazole activity too, others had retained tinidazole activity and were clearly more tinidazole selective than NfsA_Ec.

For the sake of comparison, metronidazole and tinidazole SOS assays performed on NfsA_Ec 7SM clones that had only received niclosamide selection, as opposed to both niclosamide and metronidazole selection (as in Figure 3.14) is displayed in Appendix Figure 8.3 The results demonstrate that when NfsA_Ec 7SM mutants were only selected using niclosamide, their metronidazole and tinidazole SOS responses tended to parallel each other, either both increasing or decreasing. Taken together these observations demonstrated that the presence of metronidazole in the LB agar was indeed necessary to recover NfsA_Ec 7SM mutants that had diminished metronidazole activity.

The nine most promising mutants identified over the course of the library screening were taken forward for further analysis. These mutants were selected because they induced lower metronidazole SOS responses than NfsA_Ec, but retained comparable, albeit slightly lower, tinidazole SOS responses. To ensure the changes in prodrug selectivity were driven by mutations in the NfsA_Ec gene, and not due to acquired genomic mutations (a phenomenon previously observed by the Ackerley lab), the pUCX plasmids containing each mutant were isolated and retransformed into fresh $E$. coli SOS-R4 cells. To demonstrate the tinidazole selectivity of these retransformed mutants relative to NfsA_Ec, the metronidazole and tinidazole SOS responses of each of the selected mutants are presented in Figure 3.15. 


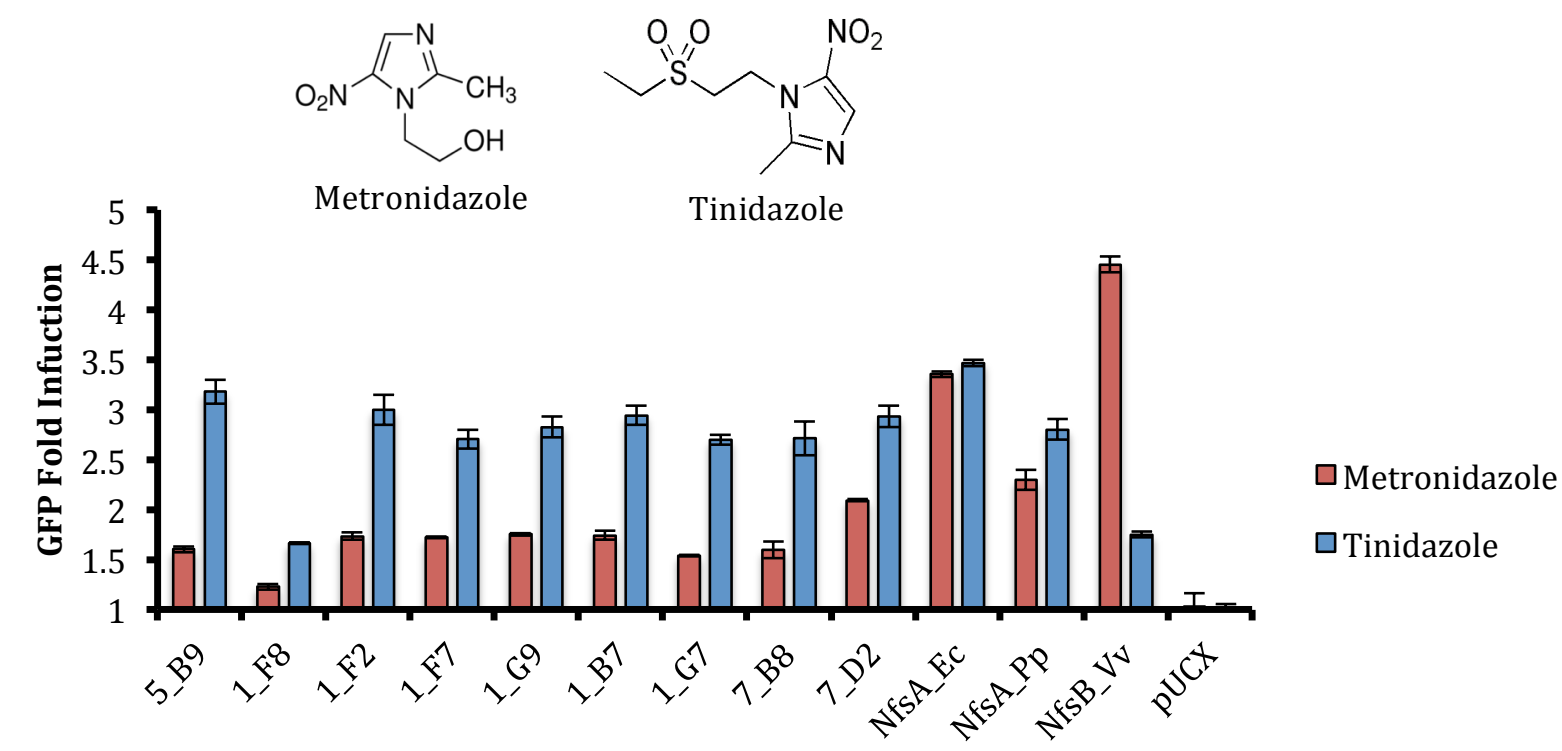

NfsA_Ec 7SM Mutant

Figure 3.15 - Metronidazole and tinidazole GFP SOS assays of the top 7SM mutants selected with metronidazole and niclosamide. SOS-R4 cells expressing the nine top tinidazole selective NfsA_Ec 7SM mutants strains were challenged with $5 \mu \mathrm{M}$ metronidazole or $5 \mu \mathrm{M}$ tinidazole for 3 hours, after which GFP fluorescence $(488 \mathrm{ex} / 510 \mathrm{em})$ and culture turbidity $\left(\mathrm{OD}_{600}\right)$ were recorded. Unchallenged DMSO (vehicle) controls were also included for each of the E. coli SOS-R4 strains. The raw GFP values of each challenged culture and their respective unchallenged control were normalized by $\mathrm{OD}_{600}$. GFP fold induction was then calculated from the difference between the normalized GFP values of each challenged culture and its respective unchallenged control. pUCX represents E. coli SOS-R4 cells containing an empty pUCX plasmid. E. coli SOS-R4 cells expressing NfsA_Ec and NfsA_Pp from pUCX plasmids were included as tinidazole selective control. E. coli SOS-R4 cells expressing NfsB_Vv from a pUCX plasmid were included as a metronidazole selective control. Data are representative of three independent experiments \pm SEM.

On the basis of the relative metronidazole and tinidazole SOS values, all of the NfsA_Ec 7SM mutants appeared more tinidazole selective than NfsA_Ec and NfsA_Pp. The NfsA_Ec 7SM mutant 5_B9 looked particularly promising as it demonstrated the largest relative decrease in metronidazole SOS compared to its tinidazole SOS response. Also notable, the process of retransformation had decreased the tinidazole SOS response of NfsA_Ec 7SM mutant 1_F8 from that previous observed (where it was similar to that of the other eight mutants in Figure 3.15).

Overall these experiments demonstrated that LB agar containing niclosamide and metronidazole was a successful directed evolution strategy for eliminating nitroreductase metronidazole activity, thereby enhancing tinidazole selectivity. Furthermore, as illustrated by comparing Figure 3.14 and Appendix Figure 8.2, the loss of 
metronidazole activity was the directed result of negative selection using metronidazole, rather than being directly promoted by the niclosamide positive selection.

\section{Sequence analysis of Top NfsA_Ec 7SM mutants}

We next sought to determine if the shared improvements in tinidazole selectivity demonstrated by the nine top NfsA_Ec 7SM mutants selection (those displayed in Figure 3.15) were reflected by common amino acid changes. This was achieved by sequencing the each of the nine NfsA_Ec 7SM mutants. The amino acid changes present at each of the seven sites are presented in in Table 3.2.

\begin{tabular}{|c|c|c|c|c|c|c|c|}
\hline \multirow{2}{*}{$\begin{array}{l}N f S A \_E c \\
7 S M \text { Mutal }\end{array}$} & \multicolumn{7}{|c|}{ Amino Acid Change at Mutated Codon Site } \\
\hline & 41 & 42 & 83 & 222 & 224 & 225 & 227 \\
\hline 5_B9 & $\mathrm{Y}$ & $\mathrm{C}$ & $\mathrm{L}$ & $\mathrm{H}$ & $\mathrm{C}$ & G & I \\
\hline 1_F8 & $\mathrm{R}$ & $\mathrm{H}$ & $\mathrm{R}$ & $\mathrm{E}$ & G & $\underline{\mathrm{R}}$ & $\mathrm{R}$ \\
\hline 1_F2 & $\mathrm{Y}$ & $\mathrm{Y}$ & $\mathrm{R}$ & $\underline{\mathrm{K}}$ & $\mathrm{L}$ & $\mathrm{N}$ & $\mathrm{Y}$ \\
\hline 1_F7 & Y & $\mathrm{C}$ & $\mathrm{L}$ & $\mathrm{R}$ & $\mathrm{L}$ & $\mathrm{S}$ & I \\
\hline 1_G9 & $\mathrm{Y}$ & $\mathrm{C}$ & $\mathrm{L}$ & $\mathrm{R}$ & $\mathrm{L}$ & $\mathrm{S}$ & I \\
\hline 1_B7 & Y & I & $\underline{F}$ & $\mathrm{R}$ & G & V & I \\
\hline 1_G7 & $\mathrm{F}$ & $\mathrm{C}$ & $\mathrm{L}$ & $\mathrm{R}$ & $\mathrm{R}$ & $\mathrm{Y}$ & $\underline{F}$ \\
\hline 7_B8 & $\mathrm{F}$ & $\mathrm{C}$ & $\mathrm{L}$ & $\mathrm{R}$ & $\mathrm{V}$ & Y & $\mathrm{H}$ \\
\hline 7_D2 & $\mathrm{H}$ & $\mathrm{C}$ & $\mathrm{L}$ & $\mathrm{V}$ & $\mathrm{L}$ & $\mathrm{H}$ & $\mathrm{V}$ \\
\hline NfsA_Ec & $\underline{\mathrm{S}}$ & $\underline{F}$ & $\underline{F}$ & $\underline{\mathrm{K}}$ & $\underline{\mathrm{S}}$ & $\underline{\mathrm{R}}$ & $\underline{F}$ \\
\hline
\end{tabular}

Table 3.2 - Amino acid changes present in the top tinidazole selective NfsA_Ec 7SM mutants.

The table presents the amino acid present at each of the codon mutagenesis positions targeted in the NfsA_Ec 7SM library. The amino acids present at these same sites in NfsA_Ec are included for comparison. Amino acids present in the NfsA_Ec 7SM mutants identical to wild type are underlined. 
The results from the sequencing revealed each of the selected 7SM NfsA_Ec mutants contained a unique set of seven codon changes, although several common changes were present. The transition from a serine at position 41 to a hydrophobic/aromatic amino acid such as tyrosine, phenylalanine, or histidine was present in all mutants sequenced. The amino acid changes such as F42C and F83L were also common, found in six out the nine mutants. Overall, however, more mutant sequences and a comprehensive investigation of structure-activity relationships would be required to determine the underlying reasons for the observed changes in substrate selectivity.

\subsubsection{Metronidazole and Tinidazole $\mathrm{IC}_{50}$ values of top 7SM mutants}

To validate the SOS data (Figure 3.15), which indicated that the selected NfsA_Ec 7SM mutants had improved tinidazole selectivity, metronidazole and tinidazole $\mathrm{IC}_{50}$ values were calculated for SOS-R4 cells expressing each of the top nine mutants Table 3.3. 


\begin{tabular}{|c|c|c|c|c|c|c|}
\hline Enzyme & $\begin{array}{l}\text { Metronidazole } \quad I C_{50} \\
(\mu M)\end{array}$ & $\begin{array}{ll}\text { Tinidazole } & I C_{50} \\
(\mu M) & \end{array}$ & $\begin{array}{l}\text { Tinidazole Selectivity } \\
\text { (Metronidazole } \\
I C_{5} \text { /Tinidazole } I C_{50} \text { ) }\end{array}$ & $\begin{array}{l}\text { Fold Increase in } \\
\text { Metronidazole IC } C_{50} \\
\text { Relative to } N f S A \_E c\end{array}$ & $\begin{array}{l}\text { Fold Increase in Tinidazole } \\
I_{50} \text { Relative to } N f_{S} A_{-} E c\end{array}$ & $\begin{array}{l}\text { Fold Increase in Tinidazole } \\
\text { Selectivity Relative to } \\
N f_{S}{ }_{-} E c\end{array}$ \\
\hline \multicolumn{7}{|l|}{ Wild type } \\
\hline NfsA_Ec & $55 \pm 3$ & $30 \pm 2$ & 2 & - & - & - \\
\hline NfsA_Pp & $240 \pm 23$ & $80 \pm 6$ & 3 & 5 & 3 & 2 \\
\hline NfsB_Vv & $29 \pm 2$ & $380 \pm 29$ & 0.1 & 0.5 & 13 & - \\
\hline \multicolumn{7}{|l|}{$\begin{array}{l}7 S M \\
\text { Mutants }\end{array}$} \\
\hline 5_B9 & $1700 \pm 150$ & $80 \pm 8$ & 21 & 31 & 3 & 12 \\
\hline 1_F8 & $3900 \pm 280$ & $590 \pm 68$ & 7 & 71 & 20 & 4 \\
\hline 1_F2 & $1800 \pm 54$ & $110 \pm 12$ & 16 & 32 & 4 & 9 \\
\hline 1_F7 & $1000 \pm 53$ & $120 \pm 8$ & 9 & 19 & 4 & 5 \\
\hline 1_G9 & $1000 \pm 140$ & $100 \pm 8$ & 10 & 18 & 3 & 6 \\
\hline 1_B7 & $680 \pm 20$ & $74 \pm 7$ & 9 & 12 & 3 & 5 \\
\hline 7_B8 & $2800 \pm 130$ & $140 \pm 14$ & 20 & 50 & 5 & 11 \\
\hline 1_G7 & $1700 \pm 71$ & $200 \pm 19$ & 8 & 30 & 7 & 4 \\
\hline 7_D2 & $1400 \pm 200$ & $170 \pm 19$ & 9 & 26 & 6 & 5 \\
\hline pUCX & $>6000 \mu \mathrm{M}$ & $>800 \mu \mathrm{M}$ & & & & \\
\hline
\end{tabular}

Table 3.3 - Metronidazole and tinidazole $\mathrm{IC}_{50}$ assays of $\boldsymbol{E}$. coli SOS-R4 nitroreductase overexpressing strains. SOS-R4 cells containing top nine NfsA_Ec 7SM clones were challenged in 384 well plates across serial dilutions of metronidazole and tinidazole for $4 \mathrm{~h}$. Growth was measured as the difference in culture turbidity $\left(\mathrm{OD}_{600}\right)$ between pre $(\mathrm{t}=0 \mathrm{~h})$ and post $(\mathrm{t}=4 \mathrm{~h})$ drug challenged cultures. For each strain, growth inhibition was measured as the percentage decrease between challenged cultures and its respective unchallenged control. Data are the average of at least three independent experiments \pm SEM. GraphPad Prism $6^{\mathrm{TM}}$ non-linear regression analysis was used to calculate metronidazole and tinidazole $\mathrm{IC}_{50}$ values for each $\mathrm{SOS} \_\mathrm{R} 4$ strain. 
The results demonstrated that while the tinidazole $\mathrm{IC}_{50}$ value for each mutant had increased relative to NfsA_Ec, indicating a decrease in activity, in each case the metronidazole $\mathrm{IC}_{50}$ had increased by a greater extent. All of NfsA_Ec 7SM mutants were more tinidazole selective (metronidazole $\mathrm{IC}_{50} /$ tinidazole $\mathrm{IC}_{50}$ ) than either NfsA_Ec or NfsA_Pp. Consistent with the previous SOS data (Figure 3.15), the most tinidazole selective (metronidazole IC50 / tinidazole $\mathrm{IC}_{50}$ ) mutant was NfsA_Ec 7SM mutant 5_B9. From the $\mathrm{IC}_{50}$ data, 7SM mutant 5_B9 was ca. 12 fold more selective for tinidazole than metronidazole compared to NfsA_Ec. An added advantage of this mutant in particular was it possessed one of the lowest tinidazole $\mathrm{IC}_{50}$ values of the selected mutants (only 3 fold larger than that of NfsA_Ec). To more clearly visualize the increased tinidazole selectivity of 7SM mutant 5_B9 relative to its wild type progenitor NfsA_Ec, as well as its potential partner for multiplex ablation assays, $\mathrm{NfsB}_{-} \mathrm{Vv}$, metronidazole and tinidazole growth data for each enzyme is presented in Figure 3.16. Whereas the growth of E. coli SOS-R4 clones expressing NfsA_Ec was almost completely inhibited by $400 \mu \mathrm{M}$ (Figure 3.16B) metronidazole, E. coli SOS-R4 clones expressing the 7SM mutant 5_B9 still grew to $80 \%$ the cell turbidity of the unchallenged cell culture (Figure 3.16A). 7SM mutant 5_B9 had therefore clearly lost metronidazole activity compared to NfsA_Ec. However, while $800 \mu \mathrm{M}$ tinidazole was sufficient to completely inhibit the growth of NfsA_Ec, 7SM_B9 clones grew to $15 \%$ the turbidity of the unchallenged control, indicating a slight decrease in tinidazole activity. As Figure 3.16C demonstrated, however, while 7SM mutant 5_B9 may rival NfsB_Vv in terms of its prodrug selectivity, in terms of raw activity $\mathrm{NfsB}$ _Vv was more active with metronidazole than is 7SM 5_B9 with tinidazole. 

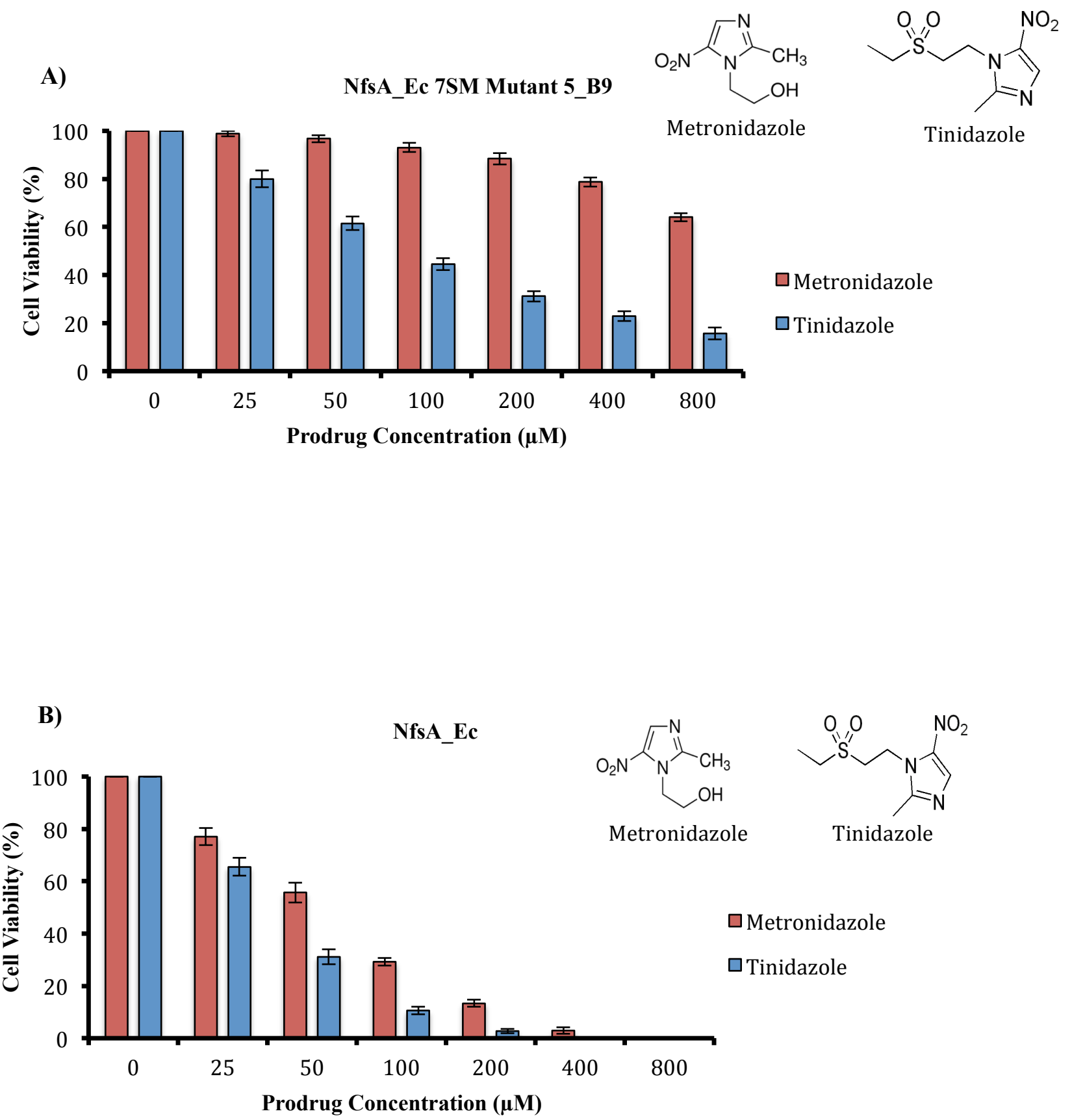


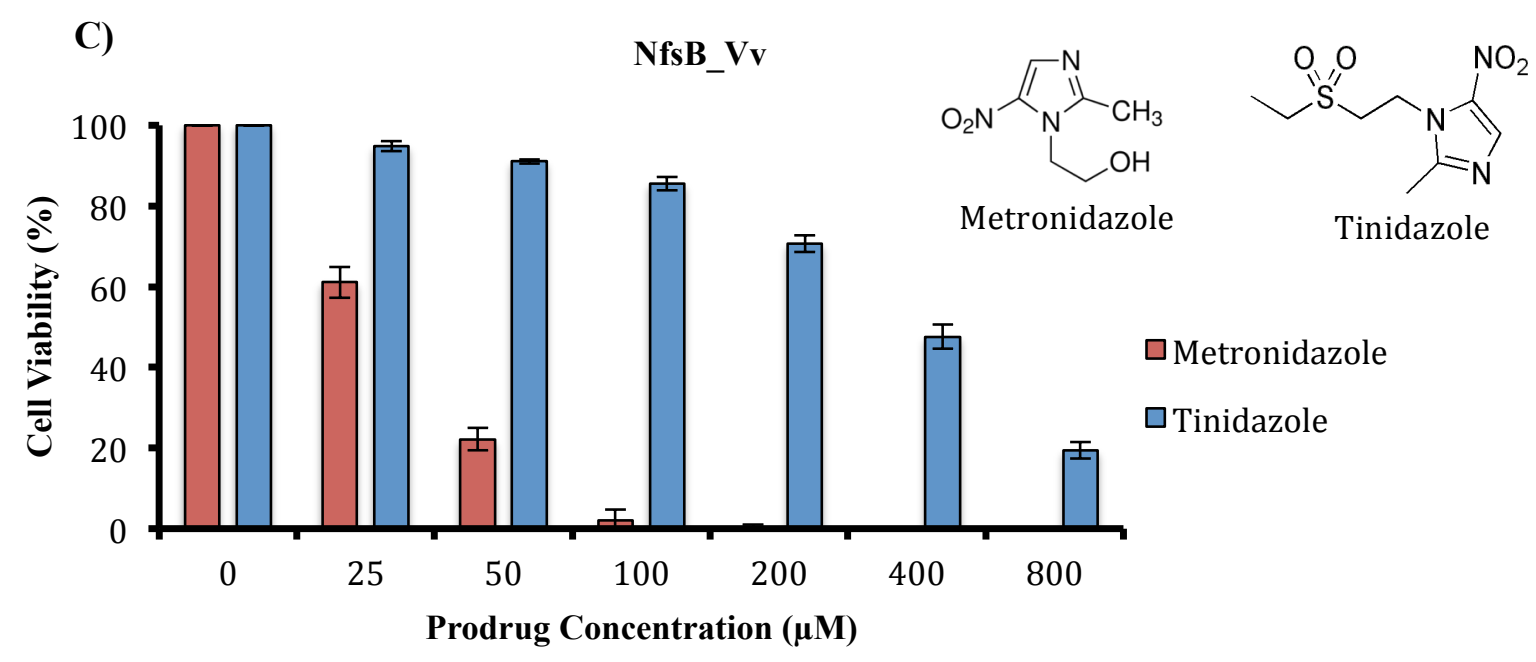

Figure 3.16 - Metronidazole and tinidazole growth inhibition assays of $E$. coli SOS-R4 nitroreductase overexpressing strains. Cultures of $E$. coli SOS-R4 overexpressing either A) lead 7SM mutant 5_B9, B) its progenitor NfsA_Ec or C) NfsB_Vv from pUCX plasmids were challenged in 384 well plates with serial dilutions of either metronidazole or tinidazole for $4 \mathrm{~h}$. Growth was measured as the difference in culture turbidity $\left(\mathrm{OD}_{600}\right)$ between pre $(\mathrm{t}=0 \mathrm{~h})$ and post $(\mathrm{t}=4 \mathrm{~h})$ drug challenged cultures. For each strain, growth inhibition was measured as the percentage decrease between challenged cultures and its respective unchallenged control. For NfsA_Ec 5_B9 tinidazole growth inhibition data are representative of seven independent experiments \pm SEM. For mutant 5_B9 metronidazole growth inhibition data are representative of four independent experiments \pm SEM. For NfsA_Ec and NfsB_Vv metronidazole and tinidazole growth inhibition data are representative of five independent experiments $\pm \mathrm{SEM}$.

\subsubsection{Expression Levels of the Top NfsA_Ec Mutants}

It was possible that some of differences in metronidazole and tinidazole $\mathrm{IC}_{50}$ values between the top nine NfsA_Ec 7SM mutants (Table 3.3) were caused by changes in protein expression, rather than changes in catalytic activity. While changes to protein expression should not affect the relative difference between the metronidazole and tinidazole $\mathrm{IC}_{50}$ of a 7SM mutant, as the two should scale accordingly, it could possibly account for mutants such as NfsA_Ec 7SM 1_F8 that possessed higher metronidazole and tinidazole $\mathrm{IC}_{50}$ values (i.e. lowest levels of activity) than any of the other mutants. To test this, an SDS-PAGE gel was run to measure the relative levels of protein expression between the top nine NfsA_Ec 7SM mutants (Figure 3.17). 


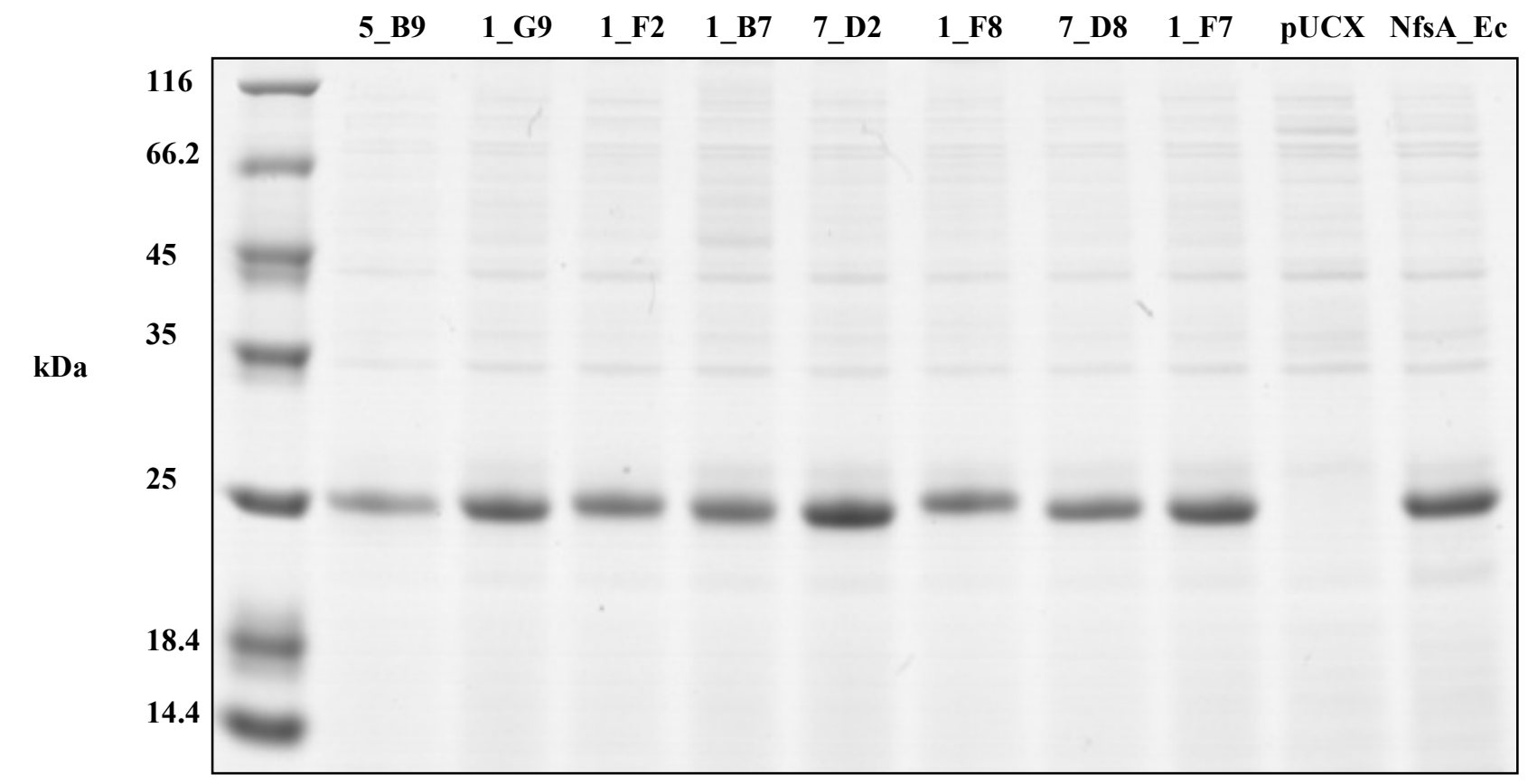

Figure 3.17 - SDS-PAGE Gel showing relative levels of protein expression of 7SM mutants. Overnight E. coli SOS-R4 cultures of each mutant strain were inoculated into fresh media and grown to an $\mathrm{OD}_{600}$ of 0.5 before protein expression was induced by addition of $0.5 \mathrm{mM}$ IPTG. Cultures were induced for 4 hours before collection. All cultures were normalized by $\mathrm{OD}_{600}$ prior to loading into a $15 \%$ SDSPAGE cell, although some small variation in loadings is evident from the variation in levels of the background E. coli proteins. E. coli SOS-R4 cells containing a pUCX plasmid were included as a negative control. NfsA_Ec $=26.8 \mathrm{kDa}$. SDS-PAGE gel is representative of two independent experiments.

From the SDS-PAGE gel, relative levels of NfsA_Ec 7SM protein expression were determined using Image $\mathrm{J}^{\mathrm{TM}}$ densitometry analysis. After correcting for the total protein present in each lane, none of the NfsA_Ec 7SM mutants demonstrated notable decreases in expression relative to NfsA_Ec (all within 85\% of wild type expression levels). From these data it was concluded that the changes in metronidazole and tinidazole $\mathrm{IC}_{50}$ values of the selected 7SM mutants relative to NfsA_Ec primarily reflect changes in NfsA_Ec catalytic activity, rather than expression level.

\subsubsection{Single Mutant Analysis of NfsA_Ec 7SM 5_B9}

We next sought to better understand the underlying structural reasons for the observed catalytic changes in substrate selectivity; in particular, if one or several of amino acid changes were primarily responsible for the improved tinidazole selectivity. By extension, amino acids that did not contribute to the improvement in tinidazole selectivity, or were even detrimental to it, could also be identified. Understanding substrate selectivity at the 
level of amino acid changes could guide the mutations introduced or avoided in future directed evolution experiments.

To determine the influence of a single codon change on tinidazole selectivity, each of the seven codon changes present in a tinidazole selective NfsA_Ec 7SM mutant was tested in isolation. Given the time constraints of this study, only the codon changes present in the most tinidazole selective NfsA_Ec 7SM mutant, 5_B9, were tested (Summarised in Table 3.4).

\begin{tabular}{|c|c|c|c|c|c|c|c|}
\hline \multirow{2}{*}{$\begin{array}{l}N f_{S A}{ }_{-} E c \\
7 S M \text { Mutan }\end{array}$} & \multicolumn{7}{|c|}{ Mutated 7SM Codon Position } \\
\hline & 41 & 42 & 83 & 222 & 224 & 225 & 227 \\
\hline 5_B9 & Y & $\mathrm{C}$ & $\mathrm{L}$ & $\mathrm{H}$ & $\mathrm{C}$ & G & I \\
\hline NfsA_Ec & $\mathrm{S}$ & $\mathrm{F}$ & $\mathrm{F}$ & K & $\mathrm{S}$ & $\mathrm{R}$ & $\mathrm{F}$ \\
\hline
\end{tabular}

Table 3.4 - Codon changes present in NfsA_Ec 7SM mutant 5 _B9.

Fortuitously, from previous work Dr. Elsie Williams had already created NfsA_Ec single mutants containing the equivalent $\mathrm{S} 41 \mathrm{Y}$ or $\mathrm{R} 225 \mathrm{G}$ changes. The rest were created in this study using overlap PCR site-directed mutagenesis. To determine the effect each of each single mutation on tinidazole selectivity, metronidazole and tinidazole growth inhibition experiments were performed using E. coli SOS-R4 cells containing a pUCX plasmid expressing each one of the single mutants in turn. Results from the growth inhibition assays are presented in Figure 3.18A and Figure 3.18B. While several amino acid changes clearly affected both metronidazole and tinidazole activity, such as F83L, none were exclusively responsible for the large increase in tinidazole selectivity observed in NfsA_Ec 7SM 5_B9. Collectively, the results indicated that the increase in tinidazole selectivity was caused by a synergistic interaction between some or all of the amino acid changes, rather than one in particular. 
A)<smiles>Cc1ncc([N+](=O)[O-])n1CCO</smiles>

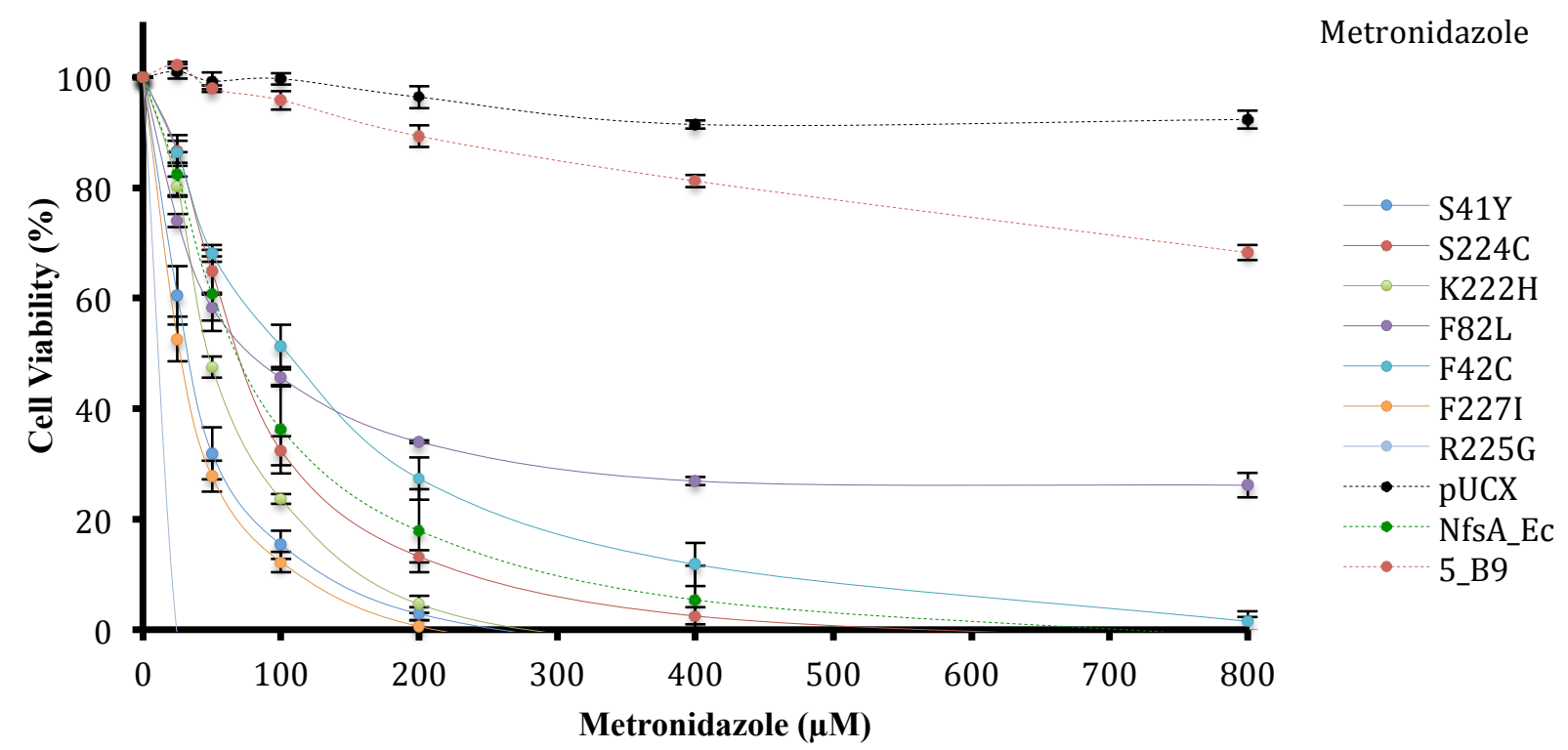

B)

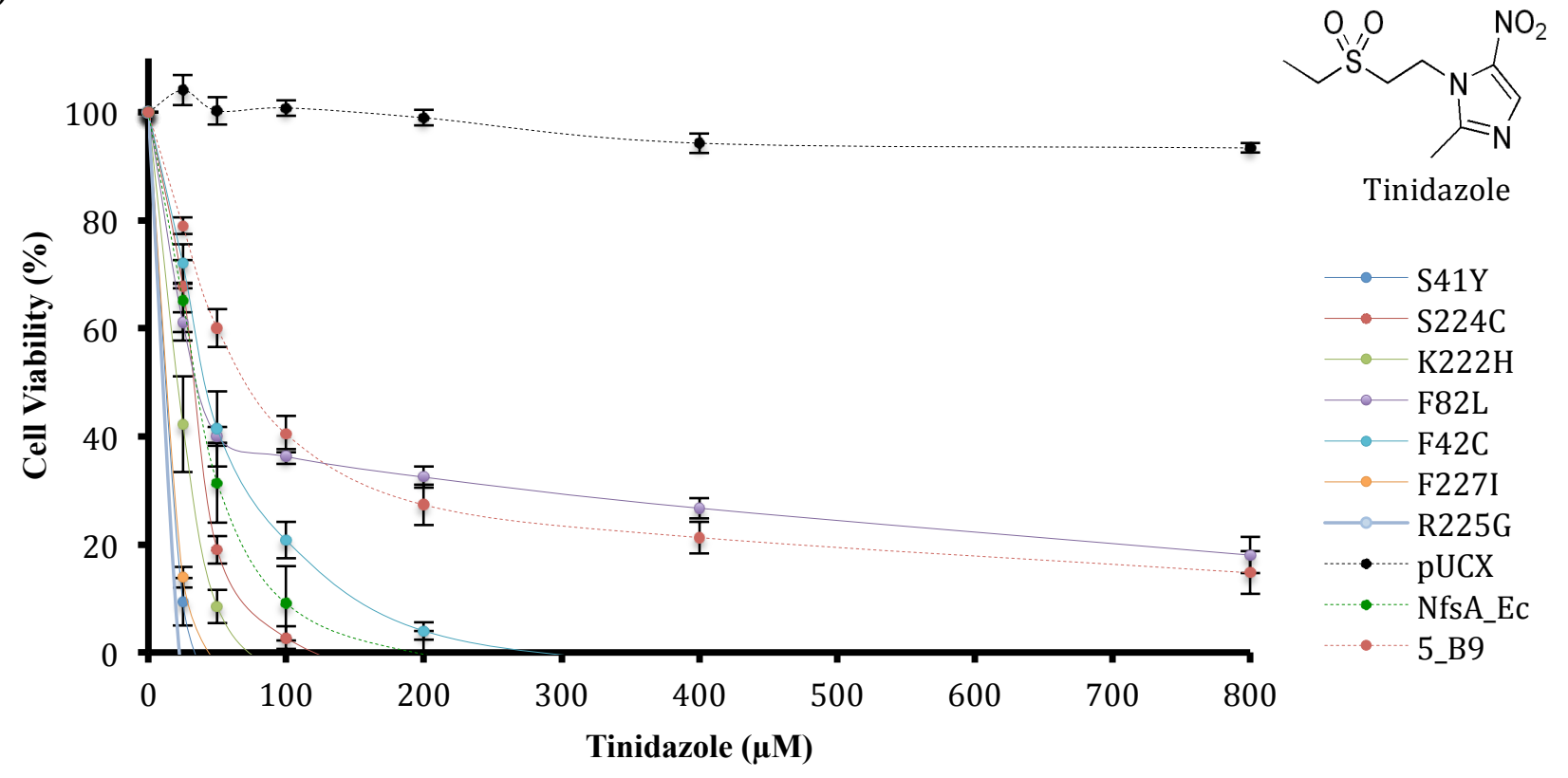

Figure 3.18 - Metronidazole and tinidazole growth inhibition assays of $E$. coli SOS-R4 NfsA_Ec single mutant overexpressing strains. E. coli SOS-R4 cultures containing either pUCX (empty plasmid control), pUCX $n f S A \_E c$, pUCX NfsA_Ec 7SM 5_B9, or pUCX $n f s A \_E c$ containing one of the seven codon changes present in 7SM 5_B9 mutant, were challenged in 384 well plates with serial dilutions of A) Metronidazole or B) Tinidazole for $4 \mathrm{~h}$. Growth was measured as the difference in culture turbidity $\left(\mathrm{OD}_{600}\right)$ between pre $(\mathrm{t}=0 \mathrm{~h})$ and post $(\mathrm{t}=4 \mathrm{~h})$ drug challenged cultures. For each strain, growth inhibition was measured as the percentage decrease in growth between each challenged culture and its respective unchallenged control. For each prodrug, data are representative of three independent experiments \pm SEM. 


\subsubsection{Multiplex Imaging and Ablation}

A potential extension of a multiplex ablation system was to also incorporate a multiplex imaging component. Analogous to prodrugs, profluorophores are a class of nitroaromatic compounds that, upon nitroreduction, can fluoresce at visible wavelengths. A wide range of nitroaromatic profluorophores have been developed, possessing emission wavelengths ranging from blue (Prosser et al., 2013; Su et al., 2013), to green (Bai et al., 2007; Cui et al., 2011), to red (Lee et al., 2013; Thorne et al., 2009), to near infrared (Bhaumik et al., 2012; Guo et al., 2013; Shi et al., 2013).

Imaging cell death caused by a prodrug is often an important component of zebrafish cell ablation experiments (Curado et al., 2008). Nitroreductase expression has been previously imaged by fusing the nitroreductase enzyme to a fluorescent protein (Curado et al., 2007; Hsu et al., 2010), or by fluorescent protein co-expression from a bicistronic promoter (Lee et al., 2012; Li et al., 2012). There is some evidence, however, that profluorophores could be superior to fluorescent proteins for detecting nitroreductase expression. For example, when compared to GFP transgene imaging, the near-infrared profluorophore CytoCy5S demonstrated greater detection, sensitivity and resolution of cancerous tissue expressing NfsB_Ec in mice (McCormack et al., 2013). Profluorophores are starting to be used in zebrafish studies as well, with a red-emitting profluorophore recently being used to detect nitroreductase expression in zebrafish embryos (Li et al., 2015). Adopting profluorophores in place of fluorescent proteins could therefore improve the imaging of transgenically expressed nitroreductase proteins in future cell ablation experiments.

Profluorophores offer an additional opportunity compared to fluorescent proteins arising from the fact that, like prodrugs, they are nitroreductase substrates. Just as nitroreductase enzymes demonstrate a bias towards activating certain prodrugs, so too can they demonstrate a bias for one profluorophore over another (Condon, 2013; Horvat, 2012). Our Auckland Cancer Society Research Centre collaborators have provided a library of 54 nitroaromatic profluorophores (referred to as the ACSRC library), capable of emitting blue, green, or red wavelengths, to the Ackerley lab (Figure 3.19). 


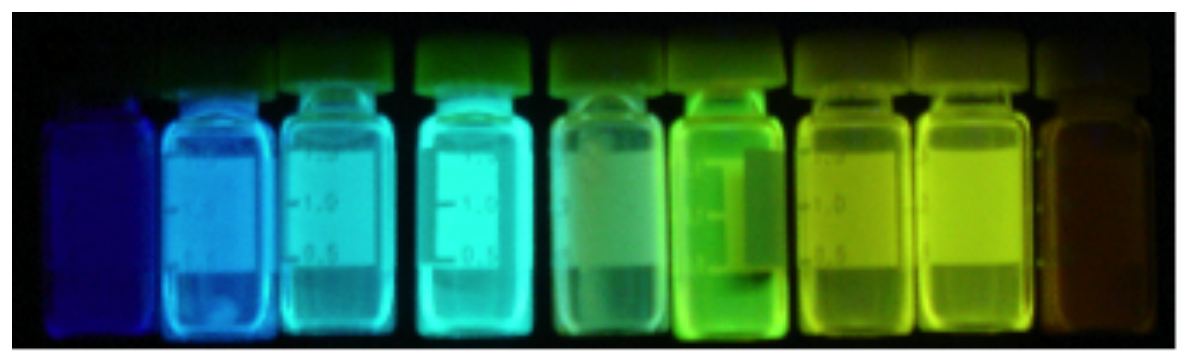

Figure 3.19 - Representation of some of profluorophores available in the ACSRC library.

A broad wavelength transilluminator was used to excite a range of different colour profluorophores postactivation (i.e., after reduction of the nitro group). Photo credit: Dr. Jeff Smaill (ACSRC), reproduced with permission.

To characterise the profluorophore activity profiles of the 58 core nitroreductase library, previous Ackerley lab $\mathrm{PhD}$ student Claire Horvat tested the activity of each with the profluorophore present in the ACSRC library (Horvat, 2012). One of motivations of that research was to identify pairs of nitroreductase enzymes possessing non-overlapping profluorophore and prodrug selectivities. Discovering such a pair could enable the development of a combined imaging and ablation multiplex system (Figure 3.20). As each nitroreductase would have unique and non-overlapping profluorophore and prodrug activities, expression from two different tissues would enable them each be independently imaged and ablated. 


\section{Transgenic Zebrafish}

1)

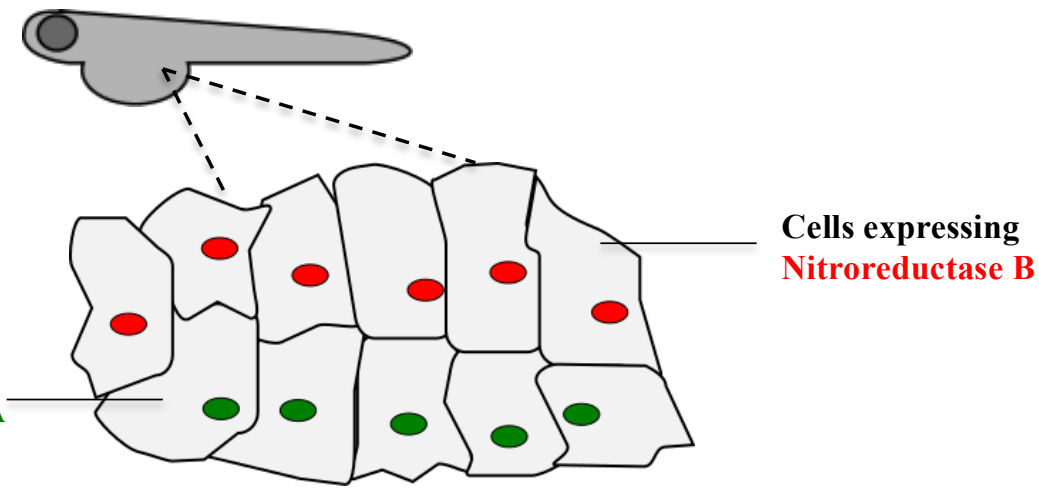

2)

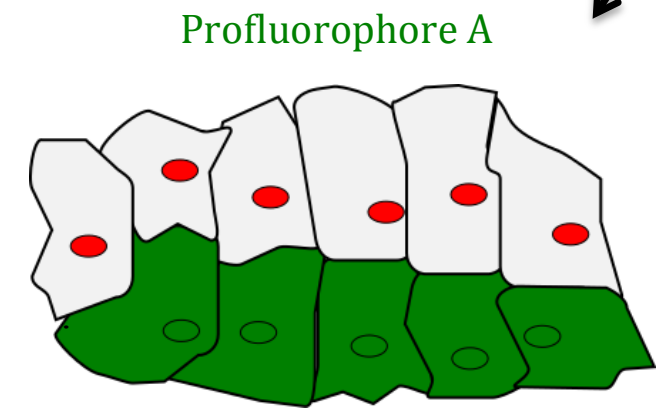

Selective imaging of Nitroreductase A expressing cells

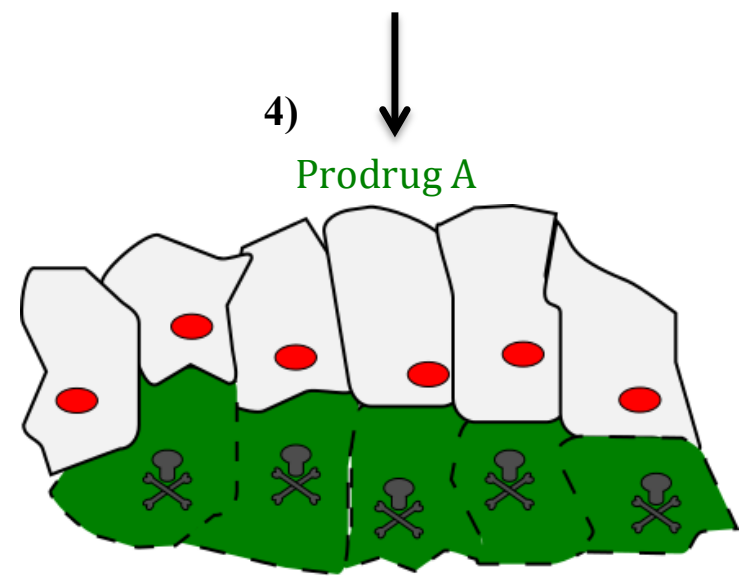

Selective ablation of Nitroreductase A expressing cells
3)
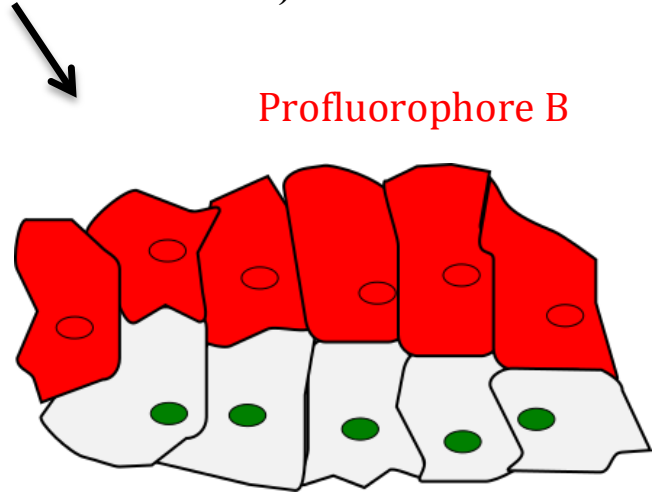

Selective imaging of Nitroreductase B expressing cells

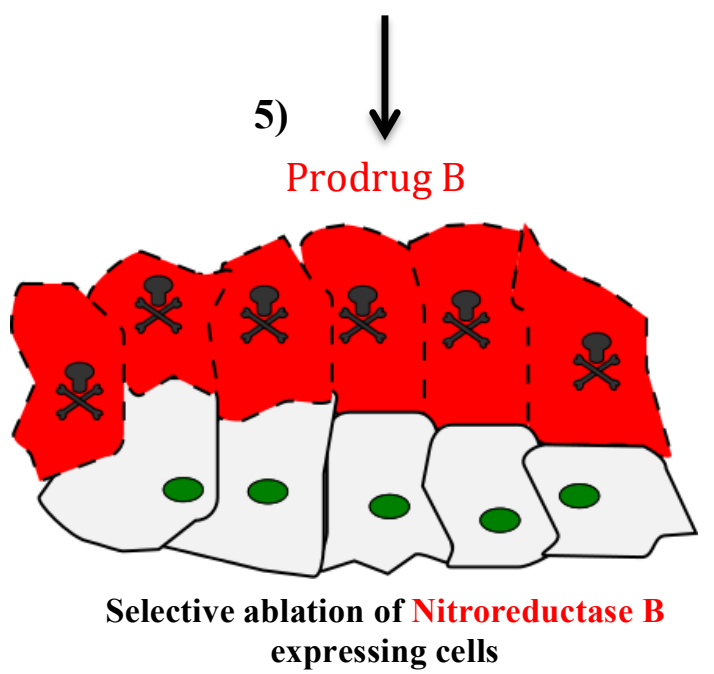

Figure 3.20 - Multiplex imaging and ablation system. 1) Two different tissues in a transgenic zebrafish each express a distinct nitroreductase enzyme possessing non-overlapping profluorophore and prodrug specificities, such that: 2) the administration of Profluorophore A enables specific imaging of cells expressing Nitroreductase A, or 3) administration of Profluorophore B enables specific imaging of cells expressing Nitroreductase B. Following profluorophore administration and nitroreductase imaging, 4) the administration of Prodrug A can specifically ablate cells expressing Nitroreductase A. 5) Alternatively, administration of Prodrug B can specifically ablate tissues expressing Nitroreductase B. Co-administration of both profluorophores could enable the simultaneous imaging of both nitroreductase-expressing tissues. 
Of particular relevance to the work performed in this thesis was the finding that NfsB_Vv (our leading metronidazole selective nitroreductase candidate) demonstrated a higher selectivity towards the red profluorophore FSL178 than to the green profluorophores FSL76 and FSL150 (Claire Horvat, 2012, Sarah Condon, 2013). Conversely, NfsA_Ec (the progenitor for the NfsA_Ec 7SM library) demonstrated a higher selectivity towards the green profluorophores FSL76 and FSL150 than to FSL178 (Claire Horvat, 2012, Sarah Condon, 2013). Structures of these profluorophores are presented in Figure 3.21.

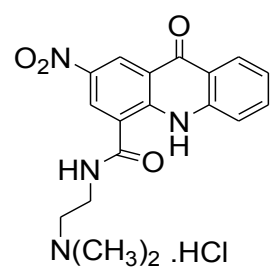

FSL76<smiles></smiles>

FSL150<smiles>Cn1c(COc2ccc3c(c2)C(C)(C)C2=C(Cl)C(=O)C(Cl)=CC2=N3)cnc1[N+](=O)[O-]</smiles>

FSL178

Figure 3.21 - Structures of the nitroaromatic profluorophores FSL76, FSL150, and FSL178. Upon nitroreduction each of these compounds can become excited and subsequently emit visible light. FSL76 and FSL150 are green profluorophores, which, once reduced, can be excited and fluoresce green light. FSL178, on the other hand is a red profluorophore that once activated by a nitroreductase can be excited and fluoresce red light. The activation of FSL178 occurs via a cleavage of the 2-nitroimidazole group upon nitroreducion in a bioreductive trigger mechanism.

Based on these previous findings, we tested if the top tinidazole selective mutants identified in the previous chapter (those in Table 3.3) could still activate FSL76 and FSL150 like their progenitor NfsA_Ec. If this were the case, then tissues expressing these NfsA_Ec 7SM mutants could be specifically imaged using FSL76 or FSL150, followed by specific ablation using tinidazole. Alternatively, neighboring tissues expressing NfsB_Vv could be specifically imaged using FSL178 and ablated using metronidazole. To test whether the top NfsA_Ec 7SM mutants could still activate FSL76 and 150, whole cell fluorescence assays were run using each profluorophore. To be consistent with previous fluorescence assays performed by the Ackerley lab, the E. coli $7 \mathrm{KO}$ strain (seven endogenous nitroreductase chromosomal gene deletions) was selected for these assays. Results from the fluorescence assays performed using the NfsA_Ecselective green profluorophores, FSL76 and FSL150, are presented in Figure 3.22. While the NfsA_Ec/NfsB_Vv profluorophore activities were as previous observed, the top tinidazole-selective NfsA_Ec 7SM mutants had lost essentially all activity with both 
FSL76 and FSL150 relative to NfsA_Ec, and in most cases were even less active than $\mathrm{NfsB}$ _Vv with these compounds. From these data we concluded that neither of these profluorophores could therefore be used to specifically image the expression of these mutants in a multiplex imaging and ablation context. 
A)
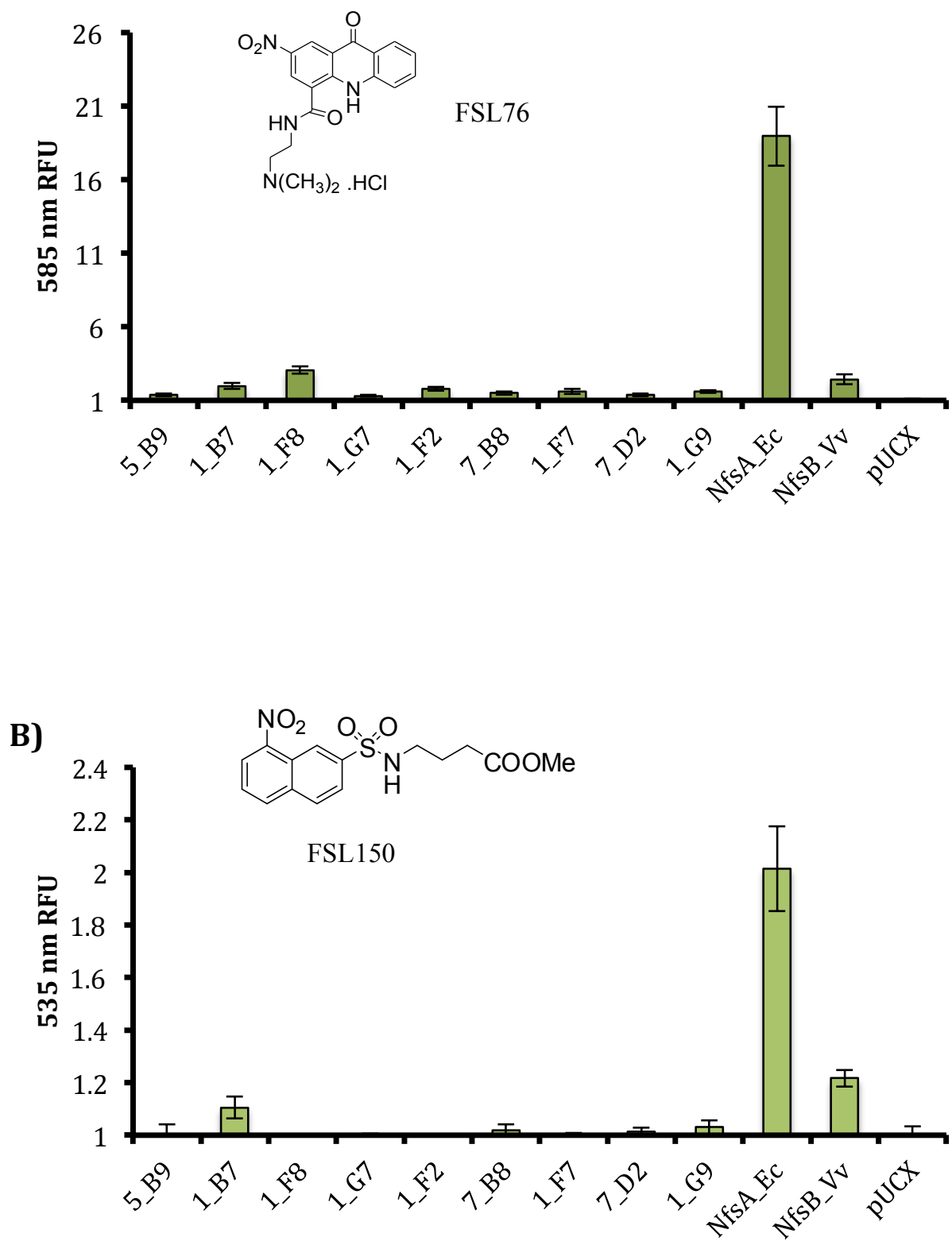

Figure 3.22 - FSL76 and FSL150 fluorescence assay with $E$. coli 7 KO cells overexpressing metronidazole-insensitive 7SM NfsA_Ec mutant nitroreductases. Mid-exponential phase E. coli 7KO strains expressing the nine top tinidazole-selective NfsA_Ec 7SM mutants identified were exposed to A) 10 $\mu \mathrm{M}$ FSL76 or B) $10 \mu \mathrm{M}$ FSL150, or to a DMSO control, for $1 \mathrm{~h}$. After the incubation period, green fluorescence (FSL76 ex 405/em 585 nm, FSL150 ex 355/em $535 \mathrm{~nm}$ ) and culture turbidity (OD 600 ) were recorded for each mutant strain. The fluorescence values of cultures exposed to profluorophore and their respective DMSO controls were normalized for $\mathrm{OD}_{600}$. Fold difference between the normalized profluorophore and DMSO controls was calculated to generate relative fluorescence units (RFU) for each 7KO strain. The wild type enzymes NfsA_Ec and NfsB Vv, expressed from pUCX in $7 \mathrm{KO}$ cells, were included as controls. pUCX represents $7 \mathrm{KO}$ cells containing an empty pUCX plasmid (lacking nitroreductase expression). FSL76 data are representative of five independent experiments \pm SEM. FSL150 data are representative of six independent experiments \pm SEM. 
Running a fluorescence assay using the red, NfsB_Vv-selective, profluorophore FSL178 revealed that the NfsA_Ec 7SM mutants had also lost activity with this profluorophore relative to wild type NfsA_Ec (Figure 3.23).

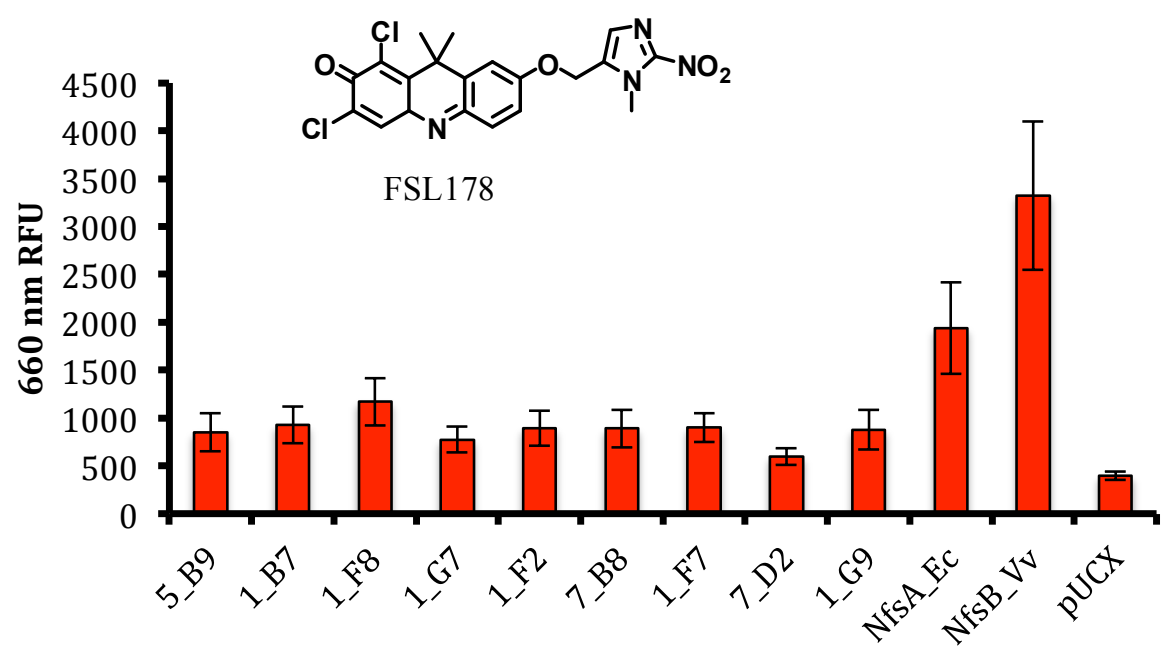

Figure 3.23 - FSL76 and FSL150 fluorescence assay with $E$. coli 7 KO cells overexpressing metronidazole-insensitive 7SM NfsA_Ec mutant nitroreductases. Mid-exponential phase E. coli 7KO strains expressing the nine top tinidazole-selective NfsA_Ec 7SM mutants identified were exposed to A) 10 $\mu \mathrm{M}$ FSL178 or a DMSO control, for $1 \mathrm{~h}$. After the incubation period, red fluorescence (ex 645/em $660 \mathrm{~nm}$ ) and culture turbidity $\left(\mathrm{OD}_{600}\right)$ was recorded for each mutant strain. The fluorescence values of cultures exposed to profluorophore and their DMSO controls were normalized for $\mathrm{OD}_{600}$. As the unchallenged controls gave fluorescence readings of 0 , fold induction could not be calculated. Data is therefore presented as $\mathrm{OD}_{600}$ normalized relative fluorescence units $(660 \mathrm{~nm}$ RFU). The wild type enzymes NfsA_Ec and $\mathrm{NfsB}$ Vv expressed from pUCX in E. coli $7 \mathrm{KO}$ cells were included as controls. pUCX represents $E$. coli $7 \mathrm{KO}$ cells containing an empty pUCX plasmid (lacked nitroreductase expression). Data are representative of three independent experiments \pm SEM.

Interestingly, the decrease in activity with FSL76, FSL150, and FSL178 demonstrated by the selected NfsA_Ec 7SM mutants was not observed with all profluorophores tested. Unlike FSL76/150 and FSL178, which are preferentially activated by NfsA or NfsB family enzymes, respectively, the blue profluorophore FSL41 is broadly active with enzymes from both families (Horvat, 2012). Relative to NfsA_Ec, none of the selected NfsA_Ec 7SM mutants had been substantially impaired in their abilities to activate FSL41 (Figure 3.24A and Figure 3.24B). However, as NfsB_Vv was also highly active with FSL41, this profluorophore could not be used to specifically image these NfsA_Ec 7SM mutants in a multiplex imaging setting. 


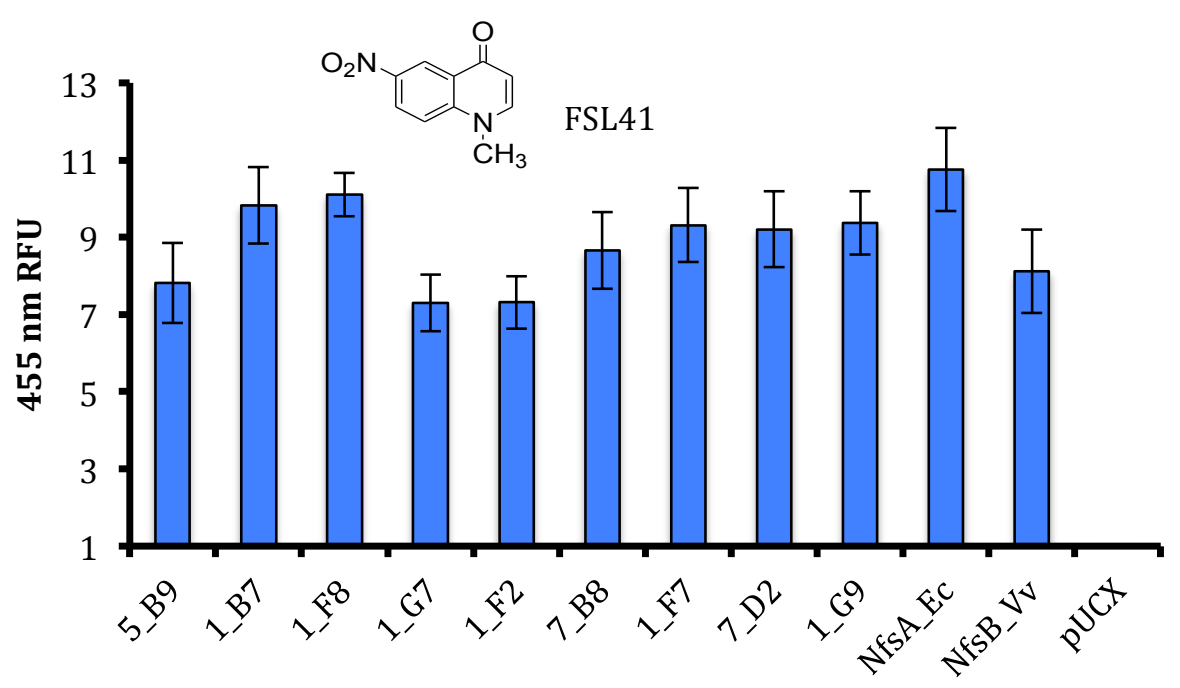

B)

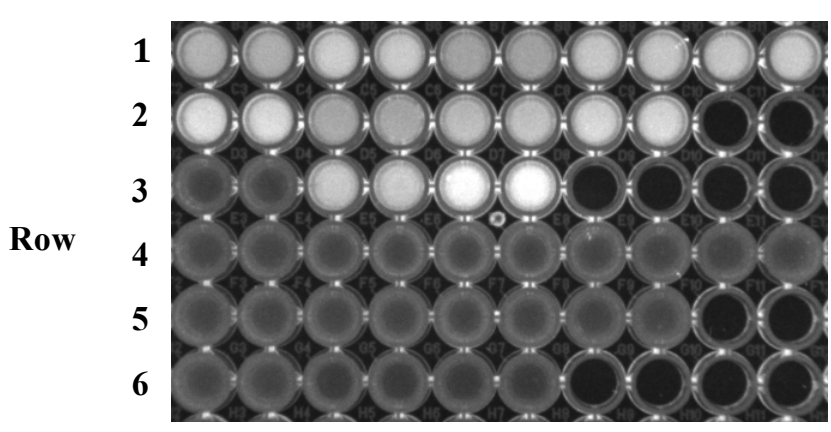

Figure 3.24 - FSL41 fluorescence assay and UV transillumination of $E$. coli $7 \mathrm{KO}$ cells overexpressing metronidazole-insensitive 7SM NfsA_Ec mutant nitroreductases. A) Mid-exponential phase E. coli 7KO strains expressing the top nine tinidazole-selective NfsA_Ec 7SM mutants identified were exposed to A) $10 \mu \mathrm{M}$ FSL41, or a DMSO control, for $1 \mathrm{~h}$. After the incubation period, blue fluorescence (ex $355 / \mathrm{em}$ $460 \mathrm{~nm})$ and culture turbidity $\left(\mathrm{OD}_{600}\right)$ was recorded for each mutant strain. The fluorescence values of cultures exposed to profluorophore and their DMSO controls were normalized for $\mathrm{OD}_{600}$. Fold difference between the normalized profluorophore and DMSO was calculated to generate relative fluorescence units (RFU) for each 7SM mutant. The wild type enzymes NfsA Ec and NfsB Vv expressed from pUCX in $7 \mathrm{KO}$ cells were included as controls. pUCX represents $7 \mathrm{KO}$ cells containing an empty pUCX plasmid. Data are representative of four independent experiments \pm SEM. B) UV transillumination image demonstrating the activation of FSL41 by the NfsA_Ec 7SM mutant overexpressing strains. After reading the blue fluorescence of one (ex 355/em $460 \mathrm{~nm}$ ) of the independent experiments, the 96 well plate in which the assay was performed was exposed to UV light (capable of exciting activated FSL41) in a transilluminator. Row 1, from left to right, duplicate wells containing: 7KO NfsA_Ec 7SM 5_B9, 1_F8, 1_F2, 1_F7 or 1_G9 cultures containing $10 \mu \mathrm{M}$ FSL41. Row 2, from left to right, duplicate wells containing: 7KO NfsA Ec 7SM 1 B 7, 1 G7, 7 B8, or 7 D2 cultures containing $10 \mu \mathrm{M}$ FSL41. Row 3, from left to right, duplicate wells containing: $7 \mathrm{KO}$ pUCX empty, NfsB_Vv, or NfsA_Ec cultures containing $10 \mu \mathrm{M}$ FSL41. Row 4, Identical layout to row 1, except an equivalent volume of DMSO present rather than $10 \mu \mathrm{M}$ FSL41. Row 5 identical layout to row 2, except an equivalent volume of DMSO present rather than $10 \mu \mathrm{M}$ FSL41. Row 6, Identical layout to row 3, except an equivalent volume of DMSO present rather than $10 \mu \mathrm{M}$ FSL41. 
Collectively, these experiments demonstrated that decreasing the metronidazole activity of NfsA_Ec using directed evolution had also been detrimental to activity with nitroaromatic substrates other than 5-nitroimidazoles. Enzyme activity was not affected with all substrates tested, however, as demonstrated by the activity of the selected NfsA_Ec 7SM mutants with FSL41. On this basis, it appeared that the activation of FSL41 by these mutants would provide a useful marker for their expression in a eukaryotic cell context, explored in the following section.

\subsubsection{Preparation for Eukaryotic $\mathrm{IC}_{50}$ analysis of the Selected NfsA_Ec 7SM mutants}

Full validation for the top tinidazole-selective NfsA_Ec 7SM mutants evolved in this research would require their expression in zebrafish and demonstrating their decreased metronidazole ablation capacity relative to NfsA_Ec. As zebrafish facilities were not readily available, however, we decided to perform preliminary validation experiments by expressing several of the NfsA_Ec 7SM mutants in eukaryotic cell cultures - HEK293 cells in this case. HEK293 cells were selected because, in addition to being amenable to plasmid transfection and heterologous protein expression (Thomas and Smart, 2005), they had previously been successfully stably transfected with $n f_{S} A_{-} P p$ and $n f s B \_V v$ genes (Condon, 2013). Consistent with the bacterial $\mathrm{IC}_{50}$ data, the HEK293 cells expressing NfsB_Vv were more sensitive to metronidazole than HEK293 cells expressing NfsA_Pp, which were more tinidazole sensitive (Condon, 2013; Horvat, 2012).

The mammalian expression plasmid 279-V5 was used to transfect HEK293 cells, having previously been created for the heterologous expression of nitroreductase genes in HCT116 and HEK293 cells (Condon, 2013; Horvat, 2012; Prosser et al., 2013; Williams, 2013). The NfsA_Ec 7SM mutants 5_B9, 1_G9, 1_B7 and 1_F2 were selected for cloning into the mammalian expression vector 279-V5. These mutants were selected because, in addition to all possessing higher bacterial metronidazole $\mathrm{IC}_{50}$ values than NfsA_Ec, their tinidazole $\mathrm{IC}_{50}$ values were the most similar to wild type NfsA_Ec (summarised in Table 3.5). 


\begin{tabular}{|c|c|c|}
\hline $\begin{array}{l}\text { NfSA_Ec } 7 S M \text { Mutants selectea } \\
\text { for 279-V5 cloning }\end{array}$ & Metronidazole $I C_{50}(\mu M)$ & Tinidazole $I C_{50}(\mu M)$ \\
\hline 1_G9 & $1000 \pm 140$ & $100 \pm 8$ \\
\hline 1_B7 & $680 \pm 20$ & $74 \pm 7$ \\
\hline 1_F2 & $1800 \pm 54$ & $110 \pm 12$ \\
\hline 5_B9 & $1700 \pm 150$ & $80 \pm 8$ \\
\hline \multicolumn{3}{|l|}{ Wild Type } \\
\hline NfsA_Ec & $55 \pm 3$ & $30 \pm 2$ \\
\hline
\end{tabular}

Table 3.5 - NfsA_Ec 7SM mutants selected for transfection into HEK293 cells. (Data taken from Table 3.1)

All four NfsA_Ec 7SM mutants were successfully cloned into the 279-V5 vector using Gateway $^{\mathrm{TM}}$ (Invitrogen) recombination. In addition, a 279-V5 vector containing NfsA_Ec was already available from previous studies. In separate experiments, HEK293 cells were transfected with each of these five vectors, and cell cultures were grown in the presence of puromycin to select for those cells transfected with a plasmid (which can only be maintained in these cells via genomic integration).

Stably puromycin-resistant HEK293 cell lines were successfully created for the wild type NfsA_Ec and the NfsA_Ec 7SM mutants 1_B7, 1_G9, and 1_F2. While a stably puromycin resistant HEK293 population was created from transfecting NfsA_Ec 7SM 5_B9, contamination issues in the tissue culture facility meant the cells unfortunately needed to be destroyed. Moreover, due to time constraints it was not possible to definitively confirm expression of any of these gene variants via FSL41 fluorescence assays, nor to initiate any prodrug sensitivity testing. However, the transfected HEK293 cell lines should be a useful resource for future experiments.

\subsection{Chapter 3 Discussion}

The goal of this chapter was to improve the tinidazole selectivity of NfsA_Ec using directed evolution. For multiplex cell ablation to be possible, two nitroreductase enzymes, each possessing non-overlapping prodrug selectivities, are required. Previous work had identified several promising nitroreductase pairs demonstrating a 
of non-overlapping selectivities for the prodrugs metronidazole and tinidazole (Condon, 2013; Horvat, 2012). In particular, NfsB_Vv was identified as a highly metronidazole selective nitroreductase, while NfsA_Ec was identified as weakly tinidazole selective (Condon, 2013; Horvat, 2012). Before incorporating these enzymes into a multiplex ablation system, we anticipated that the substrate selectivity of one or both would require further narrowing. Given its success in altering the activity of nitroreductase enzymes in the past (discussed in Section 1.4.2), we chose to use directed evolution to enhance nitroreductase substrate selectivity.

Consistent with previous reports (Condon, 2013) metronidazole and tinidazole $\mathrm{IC}_{50}$ assays of $E$. coli SOS-R4 cells overexpressing NfsA_Ec demonstrated it was active with both prodrugs, possessing a slight ( 2 fold) tinidazole activation bias (Table 3.1). Also consistent with previous studies (Condon, 2013; Horvat, 2012), the metronidazole and tinidazole $\mathrm{IC}_{50}$ values $\mathrm{NfsB}+\mathrm{Vv}$ indicated it was considerably (13 fold) more metronidazole selective. As two highly prodrug selective nitroreductase enzymes would be required for a multiplex ablation system, we chose to focus on enhancing the tinidazole selectivity of NfsA_Ec to make it more complementary to the metronidazole selective NfsB_Vv.

From previous research in the Ackerley lab, the highly diverse NfsA_Ec 7SM mutant library was available for screening (described in Section 3.2.2). To improve the tinidazole selectivity of NfsA_Ec, a directed evolution screen was developed where NfsA_Ec mutants were simultaneously exposed to metronidazole and niclosamide, negative and positive nitroreductase selection agents, respectively. The rationale was that NfsA_Ec 7SM mutants highly active with metronidazole, as wild type NfsA_Ec was, would convert it into the cytotoxic form and inhibit their own growth. Niclosamide, on the other hand, was used as a positive selection agent to ensure the reason the mutants were viable in the presence of metronidazole was not because they had lost all nitroreductase activity. As demonstrated in Figure 3.7, LB agar containing $0.5 \mu \mathrm{M}$ niclosamide was toxic to $E$. coli SOS-R4 cells lacking nitroreductase expression, whereas E. coli SOS-R4 cells expressing NfsA_Ec from pUCX remained viable. To determine if using niclosamide would detrimentally affect the tinidazole activity of the selected NfsA_Ec 7SM mutants, the library was selected using niclosamide alone. The results demonstrated that, in contrast, the majority of mutants selected using niclosamide were 
more active than wild type NfsA_Ec with both tinidazole and metronidazole Figure 3.8. Outside of the evolution performed in this thesis, niclosamide could therefore have applications as a direct positive selection to improve nitroreductase activity with 5nitroimidazole prodrugs. As nitroreductase enzymes possessing superior metronidazole activation kinetics are starting to be utilized in zebrafish cell ablation studies (Chen et al., 2011; Mathias et al., 2014), niclosamide could assist the evolution of more such enzymes.

A concern with using niclosamide was that the ability of an NfsA_Ec 7SM mutant to detoxify it, and its ability to activate metronidazole or tinidazole, might not be mutually inclusive attributes. In which case, niclosamide could be killing desirable NfsA_Ec 7SM mutants that were highly tinidazole selective. As approximately only $4 \%$ of the NfsA_Ec 7SM library ( $\sim 95$ million mutants in totals) can detoxify niclosamide (Michelle Rich, unpublished), using niclosamide massively lowered the mutational diversity present for screening. Furthermore, it was unknown whether the minimal set of mutations required for the detoxification of niclosamide might ultimately restrict the maximum tinidazole selectivity achievable. While these were potential problems associated with niclosamide, its ability to select against mutants lacking metronidazole and tinidazole activity was highly valuable in this study. As Figure 3.9 demonstrated, in the absence of niclosamide selection, the majority of NfsA_Ec 7SM mutants had lost activity with both metronidazole and tinidazole. Thus, if niclosamide were left out of the LB agar during NfsA_Ec 7SM library screen, the number of mutants that would need to be screened to identify those few with activity would be much larger. Especially when considering the low throughput nature of the directed evolution screen, i.e. the need to select and grow individual mutants in liquid culture to measure metronidazole and tinidazole activity, the practicality of niclosamide selection was clear.

In the future, if niclosamide selection was found to inhibit maximum tinidazole selectivity, higher throughput methods such as FACS could be used to screen a nonniclosamide selected NfsA_Ec 7SM library. The induction of GFP expression in E. coli SOS-R4 cells expressing a nitroreductase in response to a prodrug has previously enabled their collection using FACS (Copp et al., 2014). E. coli SOS-R4 cells containing the full NfsA_Ec 7SM library could be challenged using tinidazole, followed by FACS collection of the most GFP positive cells. The collected population could then be challenged using metronidazole to sort the least GFP positive cells. If successful, the end result would be 
the enrichment of highly tinidazole selective NfsA_Ec mutants from the entire NfsA_Ec 7SM library, rather than just the minority viable in the presence of niclosamide.

In addition to niclosamide, the other component of the directed evolution screen was a negative selection agent: the nil-bystander prodrug metronidazole. The unique activity profile of AzoR_Ec (lacking metronidazole activity but capable of detoxifying niclosamide) resulted in it being a very valuable control to optimize the concentration of metronidazole used in directed evolution screen (Figure 3.10, Figure 3.11, and Figure 3.12). A metronidazole concentration was identified that, in combination with niclosamide, inhibited the growth of $E$. coli SOS-R4 cells that either lacked all nitroreductase activity (i.e., contained pUCX empty) or possessed wild type levels of activity (i.e., expressing NfsA_Ec from pUCX), while still enabling the survival of AzoR_Ec clones. In addition to AzoR and orthologues thereof, the Ackerley lab have identified several other "minor" nitroreductase families, typified by E. coli NemA (Prosser et al, 2010), E. coli MdaB (Prosser et al, 2013) and Pseudomonas aeruginosa MsuE (Green et al, 2013), that only seem to have activity with a minority of nitroaromatic substrates. It is possible that some of these other nitroreductase enzymes could similarly provide useful controls for other future directed evolution experiments. Thus, the use of AzoR_Ec in this work also demonstrated the usefulness of characterizing new nitroreductase enzymes, even if their application is unclear at the time of discovery.

After the directed evolution screen had been optimized, the NfsA_Ec 7SM library was screened. The results indicated that the screen successfully yielded NfsA_Ec mutants that possessed improved levels of tinidazole selectivity compared to NfsA_Ec (Figure 3.14). While the top 7SM NfsA_Ec mutants identified had increased metronidazole $\mathrm{IC}_{50}$ values, they were also increased, albeit to a lesser extent, in their tinidazole $\mathrm{IC}_{50}$ relative to NfsA_Ec (Table 3.3). These findings were consistent with a previous study where negatively selecting against enzyme activity with one substrate, was also detrimental to enzyme activity with other substrates (Nair and Zhao, 2008). Here, the loss of activity with both prodrugs could reflect that, as they were both 5-nitroimidazole prodrugs, enzymatic changes that affected activity with one were extremely likely to affect activity with the other. 
The NfsA_Ec 7SM mutant demonstrating the largest improvement in tinidazole selectivity,5_B9, was 12 fold more tinidazole selective than wild type NfsA_Ec (Table 3.3). Furthermore, measuring the protein expression levels of the mutants using SDSPAGE (Figure 3.17) suggested that catalytic changes, rather than expression changes, were responsible for the increase in tinidazole selectivity. One anomaly observed during the library screen was that upon retransformation into SOS-R4 cells, the tinidazole GFP values of NfsA_Ec 7SM 1_F8 had decreased from that observed pre-retransformation. The low metronidazole and tinidazole GFP responses of this mutant (Table 3.3) translated into the highest (i.e., worst) metronidazole and tinidazole $\mathrm{IC}_{50}$ values recorded for any of the selected mutants. Overall, the high metronidazole and tinidazole $\mathrm{IC}_{50}$ value of NfsA_Ec 7SM 1_F8 provided further evidence of the importance of retransforming promising mutant plasmids prior to conducting further analysis.

Measuring the Michaelis-Menten kinetic parameters $\left(\mathrm{K}_{\mathrm{M}}\right.$ and $\left.\mathrm{k}_{\mathrm{cat}}\right)$ for metronidazole and tinidazole would be a more robust measure of substrate selectivity than bacterial $\mathrm{IC}_{50}$ values. Unfortunately, calculating enzyme kinetic constants using metronidazole and tinidazole has proven difficult in the past due to the intrinsic $\mathrm{NAD}(\mathrm{P}) \mathrm{H}$ oxidase activity of nitroreductase enzymes, in particular members of the NfsA family. NAD(P)H oxidase activity has been observed in multiple nitroreductase enzymes (Cortial et al., 2010; Olekhnovich et al., 2009) and results in the consumption of $\mathrm{NAD}(\mathrm{P}) \mathrm{H}$ elections by molecular oxygen rather than the substrate under evaluation. In the case of NfsA_Ec, the consumption of $\mathrm{NAD}(\mathrm{P}) \mathrm{H}$ by $\mathrm{NAD}(\mathrm{P}) \mathrm{H}$ oxidase activity occurs so rapidly relative to reduction of the intended substrate as to preclude the measurement of metronidazole and tinidazole reduction kinetics. Previous studies overcame this problem via addition of oxygen scavenging enzymes, such as glucose oxidase, to the reaction mix (Olekhnovich et al., 2009). The Ackerley lab has recently acquired a hypoxic chamber that should enable kinetic measurements to be made in an entirely oxygen free environment. In anticipation, although not reported in the Results, the top tinidazole selective NfsA_Ec 7SM mutants (Table 3.3) were all cloned into the polyhistidine-tag protein purification vector $\mathrm{pET}-28 \mathrm{a}^{+}$. Metronidazole and tinidazole kinetic data of these mutants should therefore be readily measurable in the near future.

To determine if increases in tinidazole selectivity were associated with a similar series of amino acid substitutions, each of the top NfsA_Ec 7SM mutants from Table 3.3 were 
sequenced (Table 3.2). While several common amino acid motifs were present, it was difficult to determine any definitive relationships without obtaining the sequences of additional mutants. It would be interesting to test a S41Y/F, F42C, F83L triple mutant improved tinidazole selectivity; as these changes were present in five of the nine mutants sequenced. To identify if a key substitution was responsible for the observed increase in tinidazole selectivity, seven NfsA_Ec single mutants, each possessing a single amino acid change present in the tinidazole selective NfsA_Ec 7SM 5_B9 mutant were tested. Metronidazole and tinidazole growth inhibition assays were performed on E. coli SOS-R4 cells containing one of these single mutants to determine their relative activity with each prodrug (Figure 3.18). The results indicated that no single amino acid change was exclusively responsible for the improved tinidazole selectivity of NfsA_Ec 7SM 5_B9, indicating the synergistic influences of multiple amino acid changes were responsible. Alternative methods could be used to study the relationship between structure and activity in the future. For instance, variants of mutant NfsA_Ec 7SM 5_B9 could be created where each of the altered codons was, one at a time, reverted to its wild type identity. If codon reversion eliminated the improved selectivity of the enzyme it would implicate it as important for the change in enzyme substrate selectivity. This technique was previously used to identify minimal set of amino acid changes required for the altered substrate selectivity of aspartate aminotransferase mutants (Yano et al., 1998). Identifying the amino acid(s) most important for tinidazole selectivity over metronidazole selectivity could assist future improvement by directed evolution. For example, any codons identified as important for tinidazole selectivity could be mutated again, this time using an NNK codon degeneracy that provides access to all twenty possible residues, (rather than NDT, which only provides access to twelve), to determine if a full codon repertoire could further enhance selectivity.

In Section 3.2.10 we tested whether the top tinidazole selective NfsA_Ec 7SM mutants could be specifically imaged in a multiplex imaging and ablation system including NfsB_Vv (as depicted in Figure 3.20). The activities of the wild type enzymes NfsA_Ec and $\mathrm{NfsB} \mathrm{Vv}_{\mathrm{V}}$ with the profluorophores tested were consistent with those observed in previous studies (Condon, 2013; Horvat, 2012).. NfsA_Ec selectively activated FSL76/FSL150, whereas NfsB_Vv was only weakly active with these profluorophores. Also consistent, NfsB_Vv was more active with the red profluorophore FSL178 than NfsA_Ec (Condon, 2013; Horvat, 2012). When tested, however, the top tinidazole 
selective NfsA_Ec 7SM mutants had lost activity with FSL76, FSL150, and FSL178 relative to wild type NfsA_Ec (Figures 3.22A and Figure 3.22B). The loss of FSL76 and FSL150 activity suggested that none of the mutants would be suitable for a multiplex imaging system containing $\mathrm{NfsB}_{-} \mathrm{Vv}$, unless additional rounds of directed evolution could restore activity with one or both of these substrates. However, that all of the NfsA_Ec 7SM mutants tested were active with the blue profluorophore FSL41, offered promise that non-specific imaging capabilities would be possible to report on expression and activity of the evolved nitroreductase variants post-transfection into a eukaryotic cell line.

Time constraints unfortunately precluded the validation of the tinidazole selective NfsA_Ec 7SM mutants identified in this chapter in a model eukaryotic system. Some previous studies have indicated that the performance of a nitroreductase in a bacterial system frequently translates well to eukaryotic systems (Grove et al., 2003; Guise et al., 2007; Mathias et al., 2014; Swe et al., 2012). Particularly relevant to this project was the example where improvements in prodrug activation in a bacterial system (Guise et al., 2007), translated into improvements in prodrug activation in transgenic zebrafish tissue (Mathias et al., 2014). However, as effective expression of bacterial nitroreductases (and translation of their activities) in eukaryotic cells, has not always been achieved (Prosser et al., 2013), it will be important to validate the NfsA_Ec 7SM mutants in eukaryotic system in the near future. Our collaborators at the ACSRC have also encountered difficulties where a subset of transfected nitroreductase mutants express poorly in mammalian cells, even when the wild type expresses well (Williams, 2013). That stably puromycinresistance observed in cell lines were produced using several of the transfected NfsA_Ec 7SM mutants therefore provides no feedback on the stability of nitroreductase expression. The activation of FSL41 should, however, be a useful tool to measure the uniformity of nitroreductase expression within a population of transfected mammalian cells, either using confocal microscopy or flow cytometry. Alternatively, expression levels of the transfected nitroreductase mutants could also be assessed in future using Western blot analysis. The 279-V5 vector is capable (via an inducible translational readthrough system) of incorporating a V5 epitope tag to the expressed protein, enabling primary antibody binding and Western blot detection (Prosser et al., 2013). Using FSL41 activation or Western blotting, the expression levels of the transfected mutants should be readily measurable in the near future. 
For the development of a successful multiplex cell ablation system, absolute enzyme selectivity is not the only factor that needs to be considered. A suitable therapeutic window is required, such that the administration of metronidazole only ablates NfsB_Vv expressing cells, whereas the administration of tinidazole only ablates cells expressing NfsA_Ec 7SM 5_B9. Comparing metronidazole and tinidazole growth inhibition graphs in Figure 3.16, the tinidazole selectivity of mutant 5_B9 does not look strong enough for a sufficiently large therapeutic window between NfsB_Vv and NfsA_Ec 7SM 5_B9 cell ablation. Addition evolution is therefore required to further increase the tinidazole selectivity of NfsA_Ec 7SM 5_B9. Several strategies could be tested in the future to achieve this. To decrease metronidazole activity even further, the concentration of metronidazole added to the LB agar could be increased beyond $1 \mathrm{mM}$ during the additional rounds of mutagenesis, proving an even greater selective pressure against mutants that possess metronidazole activity. Alternatively, if increasing metronidazole negative selection was found to be too detrimental for tinidazole activity, continuously selecting for mutants that demonstrate an increase in tinidazole activity could be more successful. If the metronidazole selectivity of $\mathrm{NfsB}$ _ Vv was found to be insufficient in a zebrafish context, there is also potential for using directed evolution to enhance the metronidazole selectivity of NfsB_Vv. A mutant NfsB_Vv library could be plated LB agar containing tinidazole (rather than metronidazole as used for NfsA_Ec evolution) to select for NfsB_Vv mutants that possessed lower levels of tinidazole activity. 


\section{Chapter 4: Enhancing the Tinidazole Selectivity of NfsA_Pp using Directed Evolution}

\subsection{Introduction}

A parallel approach taken to evolve a highly tinidazole-selective nitroreductase for a multiplex ablation system was via randomly mutagenesis of NfsA_Pp. As discussed in the Chapter 3 introduction, NfsA_Pp was first identified as a promising tinidazoleselective nitroreductase when the activity of 16 different nil-bystander prodrugs, including metronidazole and tinidazole, was measured for each member of the 58 core nitroreductase library (Condon, 2013). When measured in this study, NfsA_Pp demonstrated a three fold lower tinidazole $\mathrm{IC}_{50}$ than metronidazole $\mathrm{IC}_{50}$, making it roughly two fold more tinidazole selective than NfsA_Ec (summarised in Table 4.1). We reasoned its larger initial tinidazole selectivity might translate to larger increases in selectivity being achievable using directed evolution than would be possible for NfsA_Ec, an enzyme with less intrinsic tinidazole selectivity.

\begin{tabular}{|llll|}
\hline Enzyme & Tinidazole $I C_{50}(\mu M)$ & Metronidazole $I C_{50}(\mu M)$ & $\begin{array}{l}\text { Tinidazole Selectivity }(\text { Metronidazole } \\
\left.I_{50} / \text { Tinidazole } I_{50}\right)\end{array}$ \\
NfsA_Ec & $30 \pm 2$ & $55 \pm 3$ & 2 \\
NfsA_Pp & $80 \pm 6$ & $240 \pm 23$ & 3.0 \\
\hline
\end{tabular}

Table 4.1 - Metronidazole and tinidazole $\mathrm{IC}_{50}$ assays of $E$. coli SOS-R4 strains overexpressing either NfsA_Ec or NfsA_Pp. (Data taken from Table 3.1)

Unfortunately, no site saturation mutagenesis library, such as the NfsA_Ec 7SM, was available for NfsA_Pp. A random mutagenesis approach, using an error-prone DNA polymerase to randomly mutate nucleotides throughout the gene, was therefore chosen. Once created, as performed in Chapter 3 the library of NfsA_Pp mutants was plated on LB agar plates containing metronidazole and niclosamide to select against mutants possessing substantial metronidazole activity. 


\subsection{Chapter 4 Results}

\subsubsection{Optimization of Directed Evolution Screen for NfsA_Pp}

Equivalent optimization experiments to those in Section 3.2.3 using E. coli SOS-R4 cells expressing azoR_Ec, $n f S A_{-} P p$, or empty pUCX plasmids were performed. Like NfsA_Ec, SOS-R4 cells expressing NfsA_Pp were capable of detoxifying niclosamide to a similar extent to AzoR_Ec (Figure 4.1). AzoR_Ec was therefore again selected as a control, in this case as a surrogate for an NfsA_Pp mutant that had lost metronidazole activity but could still detoxify niclosamide.

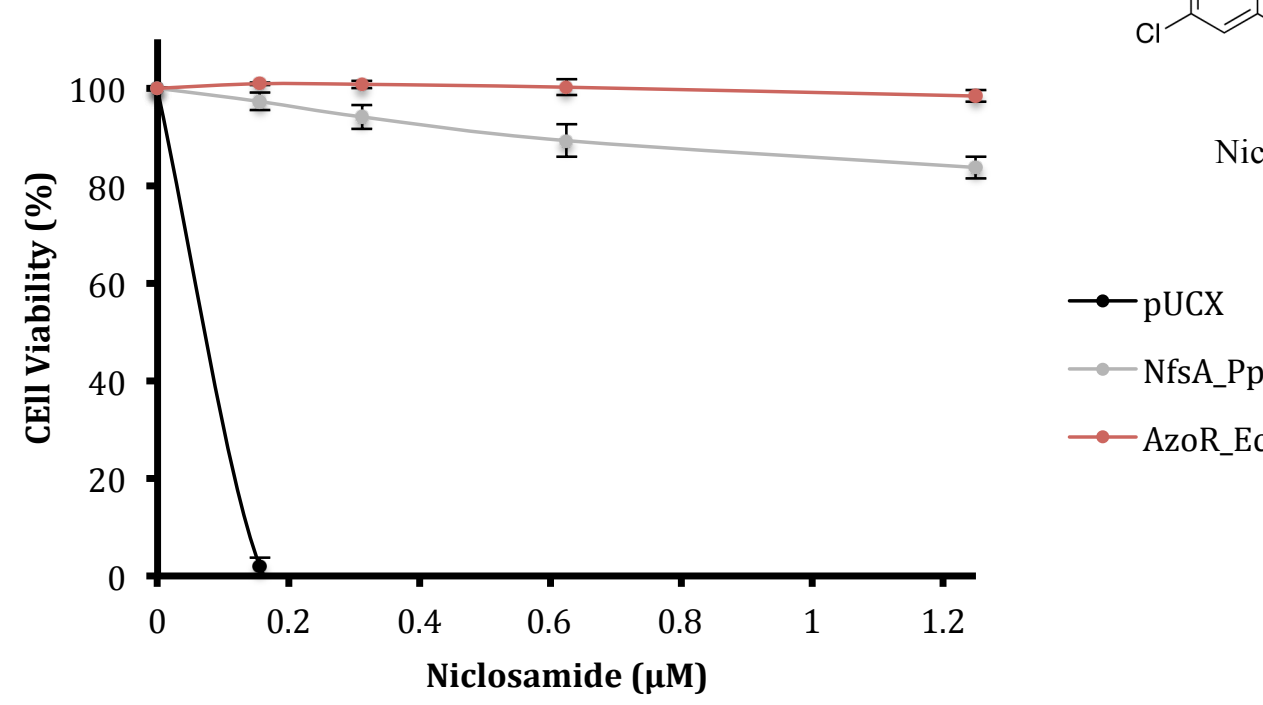

Figure 4.1 - Niclosamide growth inhibition assays of $E$. coli SOS-R4 nitroreductase overexpressing strains. E. coli SOS-R4 cultures containing expressing either NfsA_Pp or AzoR_Ec from a pUCX plasmid, or containing an empty pUCX control, were challenged in 384 well plates with serial dilutions of niclosamide for $4 \mathrm{~h}$. Growth was measured as the difference in culture turbidity $\left(\mathrm{OD}_{600}\right)$ between pre $(\mathrm{t}=0$ $\mathrm{h})$ and post $(\mathrm{t}=4 \mathrm{~h})$ drug challenged cultures. For each strain, growth inhibition was measured as the percentage decrease in growth between each challenged culture and its respective unchallenged control. Data are representative of three independent experiments \pm SEM.

After testing a range of metronidazole concentrations from $0.2 \mathrm{mM}-1 \mathrm{mM}$, LB agar supplemented with $1 \mathrm{mM}$ metronidazole and $0.5 \mu \mathrm{M}$ niclosamide was again selected as it inhibited the growth of $E$. coli SOS-R4 cells expressing NfsA_Pp from pUCX or containing an empty pUCX plasmid, but not cells expressing AzoR_Ec from pUCX. These concentrations were used in screening a random mutagenesis library of NfsA_Pp. 


\subsubsection{Preliminary Screening of a Random Mutagenesis NfsA_Pp Mutant Library}

To create a mutant library of NfsA_Pp, its gene was amplified using the error-prone DNA polymerase Mutazyme II (Stratagene). Error-prone polymerases lack the fidelity and proof reading capabilities of more conventional DNA polymerase, resulting in the introduction of base substitutions as the amplification progresses. Once created and transformed into $E$. coli SOS-R4 cells, the library was plated onto niclosamide-containing LB agar to remove nitroreductases that had lost all activity. Around $9 \%$ of the mutant library was viable on niclosamide, representing 6000 mutant clones in total. Subsequent sequence analysis revealed an average of 4-5 mutations had been introduced by the errorprone polymerase per niclosamide-resistant $n f_{S} A_{-} P p$ mutant. Niclosamide selection was once again capable of selecting for nitroreductase mutants active with both metronidazole (Figure 4.2A) and tinidazole (Figure 4.2B). In contrast with the previous 7SM targeted mutagenesis library however, in which the active site of the target (NfsA_Ec) nitroreductase had been dramatically restructured, the point mutations introduced by error prone PCR (epPCR) were more likely to slightly diminish overall nitroreductase activity; that is, a much lower proportion of the clones that were still able to defend against niclosamide were expected to be improved relative to wild type in their reduction of the 5-nitroimidazole prodrugs. 

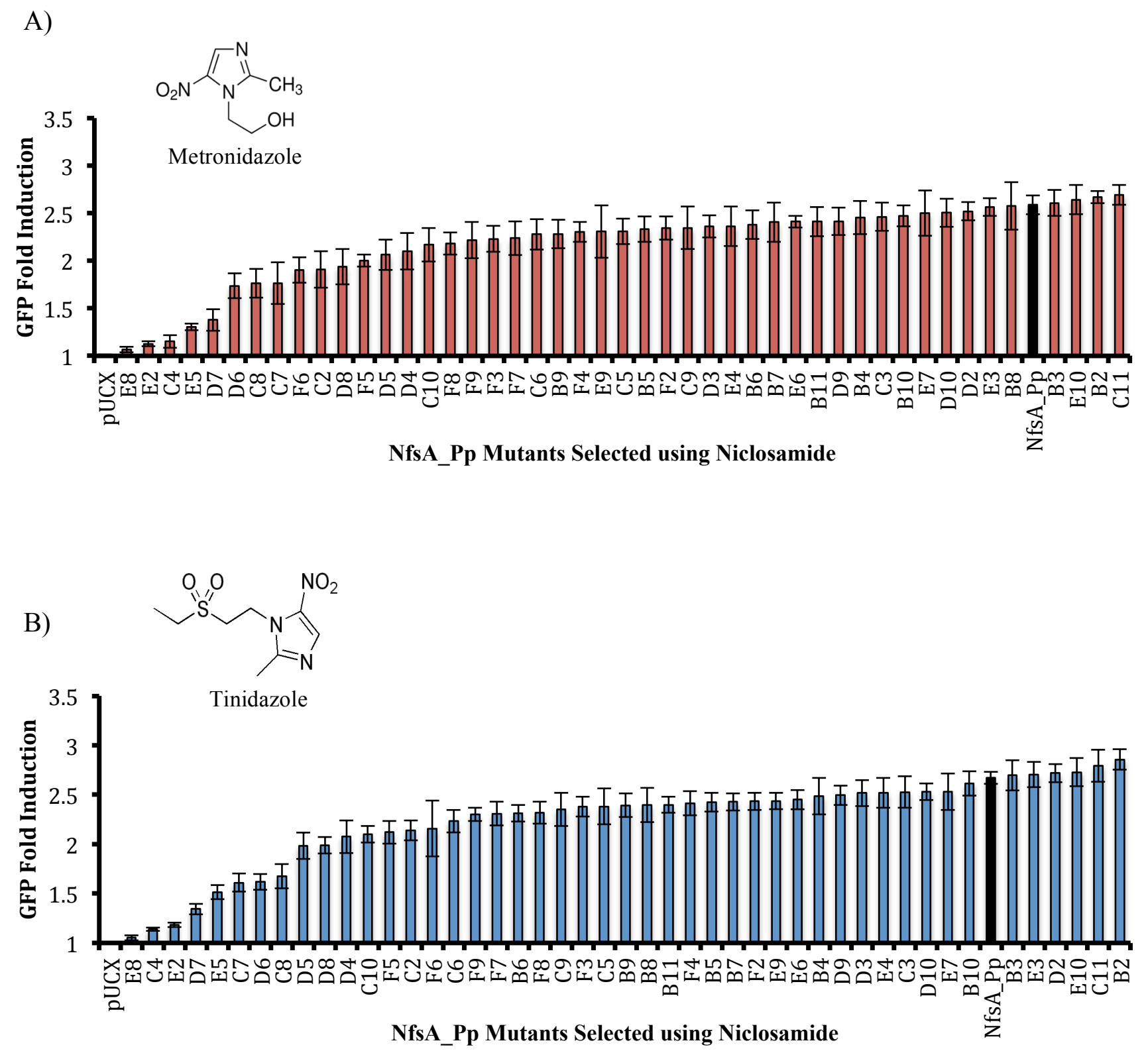

Figure 4.2 - Metronidazole and tinidazole induced GFP SOS response in niclosamide preselected $E$. coli SOS-R4 overexpressing NfsA_Pp mutants. A total of 46 NfsA_Pp mutants were randomly selected from LB agar supplemented with $0.5 \mu \mathrm{M}$ niclosamide, and then challenged using either metronidazole or tinidazole in SOS GFP assays. Mid-exponential cultures from each of the 46 colonies were challenged in 384 well plates with either A) $5 \mu \mathrm{M}$ metronidazole or B) $5 \mu \mathrm{M}$ tinidazole for 3 hours, after which GFP fluorescence $(488 \mathrm{ex} / 510 \mathrm{em})$ and culture turbidity $\left(\mathrm{OD}_{600}\right)$ were recorded. A DMSO control (unchallenged) was also included for each of the NfsA_Pp mutants. The raw GFP value for each culture was normalized by its $\mathrm{OD}_{600}$. GFP fold induction was then calculated from the difference between the normalized GFP values of each challenged culture, and its respective unchallenged control. E. coli SOS-R4 clones expressing the wild type NfsA Pp from pUCX were included for the purposes of comparison. pUCX represents E. coli SOS-R4 cells containing an empty pUCX plasmid. Data are representative of three independent experiments \pm SEM. 


\subsubsection{Mutant NfsA_Pp Library Screening}

To select for NfsA_Pp mutants that had lost metronidazole activity, the library was plated on LB agar containing $1 \mathrm{mM}$ metronidazole and $0.5 \mu \mathrm{M}$ niclosamide. Due to time constraints, library screening was not as comprehensive as that performed in Chapter 3. Of the colonies that formed in the presence of metronidazole and niclosamide, 114 were selected to run metronidazole and tinidazole SOS GFP assays. Metronidazole and tinidazole SOS GFP data for a subset (57) of the colonies screened is presented in Figure 4.3. 


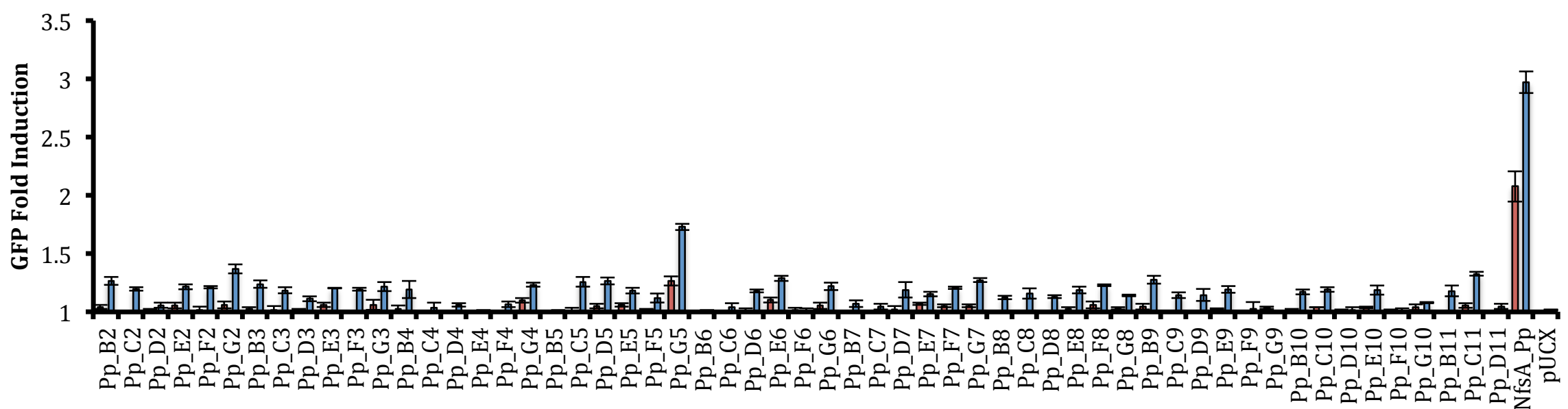

$\square$ Metronidazole

口Tinidazole

NfsA_Pp Mutants Selected With Metronidzole and Niclosamide

Figure 4.3 - Metronidazole and tinidazole induced GFP SOS response in E. coli SOS-R4 overexpressing NfsA_Pp mutant clones preselected with niclosamide and metronidazole. Mid-exponential cultures of $57 \mathrm{Nfs} A$ Pp mutants selected on LB agar containing $1 \mathrm{mM}$ metronidazole and $0.5 \mu \mathrm{M}$ niclosamide were challenged in 384 well plates containing either A) $5 \mu \mathrm{M}$ metronidazole or B) $5 \mu \mathrm{M}$ tinidazole for 3 hours, after which GFP fluorescence $(488 \mathrm{ex} / 510 \mathrm{em})$ and culture turbidity (OD 600$)$ were recorded. A DMSO control (unchallenged) was also included for each strain. The raw GFP values of each challenged culture and its respective unchallenged control were normalized by $\mathrm{OD}_{600}$. GFP fold induction was then calculated as the difference between the normalized GFP values of each challenged culture and its respective unchallenged control. E. coli SOS-R4 clones expressing the wild type NfsA Pp from pUCX were included for the purposes of comparison. pUCX represents SOS-R4 cells containing an empty pUCX plasmid control. Data are representative of three independent experiments \pm SEM. 
All of the NfsA_Pp mutants presented in Figure 4.3 had lost metronidazole activity relative to wild type NfsA_Pp, demonstrating the metronidazole negative selection had been successful. However, tinidazole activity had also been greatly decreased, to a far greater extent than observed when screening the NfsA_Ec 7SM library (Figure 3.14). The only mutant possessing notable levels of tinidazole activity was mutant Pp_G5, although from the SOS data it was difficult to determine if tinidazole selectivity had been enhanced. This mutant was therefore selected for further analysis to better characterise its change in activity.

\subsubsection{Sequence and Growth Inhibition Analysis of the top NfsA_Pp Mutant}

After retransforming the plasmid containing the Pp_G5 mutant back into E. coli SOS-R4 (as discussed in Section 3.2.5), it was sequenced to determine the mutations present. Four point mutations were present and presented in Table 4.2, two of which were missense, resulted in amino acid changes from the wild type (Q33K and V185A).

\begin{tabular}{ll}
\hline Nucleotide Position/Change & Amino Acid Position/Change \\
T94C transversion & L32 silent mutation \\
C97A transversion & Q33K missense mutation \\
G243A transversion & A81 silent mutation \\
T554C transversion & V185A missense mutation \\
\hline
\end{tabular}

Table 4.2 - Mutations identified in the NfsA_Pp mutant gene Pp_G5.

Predicting the possible catalytic consequences of these mutations was made difficult by the absence of a crystal structure. However, the E. coli homologue of NfsA_Pp, NfsA_Ec had been crystalised (Kobori et al., 2001), enabling a very perfunctory analysis to be performed. Aligning the sequence of Pp_G5 and NfsA_Ec revealed that the Q33K mutation was four amino acids distance away from the R38 codon, which is the NfsA_Pp equivalent of the R15 codon of NfsA_Ec - one of the amino acids whose side chain is present in the active site (Figure 4.4). Additionally, the V185A amino acid change is seven residues away from an equivalent NfsA_Ec amino acid, D163, which forms part of the NfsA_Ec active site. Obviously, additional experimental analysis is required to conclusively determine which mutation(s) account for the change in metronidazole and tinidazole activity observed. 


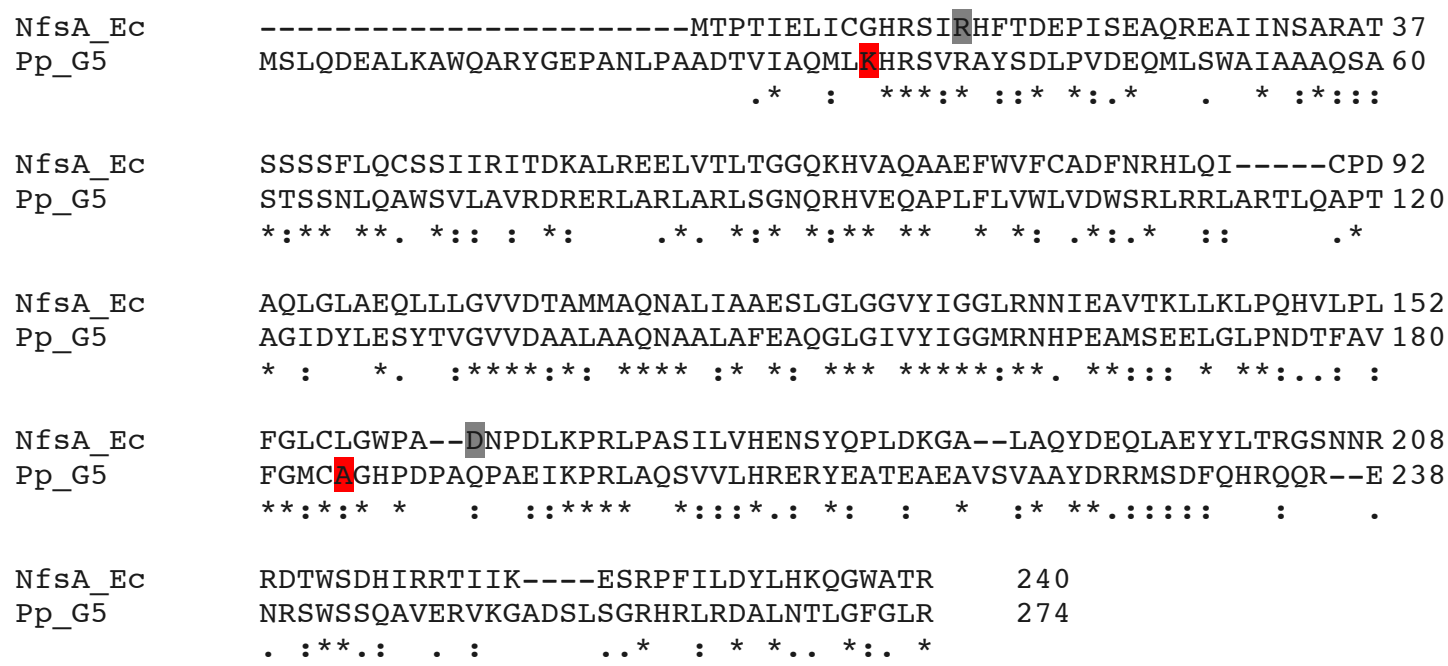

Figure 4.4 - Protein sequence alignment of NfsA_Ec and the NfsA_Pp mutant Pp_G5. The multiple sequence alignment tool ClustalOmega (Www.ebi.ac.uk/Tools/msa/clustalo/) was used to align the protein sequences of NfsA_Ec and the NfsA_Pp mutant Pp_G5. The Q33K mutation and the V185A mutations present in the Pp_G5 mutant are highlighted in red. For each NfsA_Pp mutation, the closest NfsA_Ec amino acid whose side chain forms part of the active site (determined by Kobori et al., 2001) are highlighted in grey. Conserved amino acid residues are marked with an asterisk (*). Loci with amino acids with highly similar properties (e.g. H and R) are marked with a colon (:). Loci with amino acids with weakly similar properties (e.g. Q and H) are marked with a period (.).

To validate the reduction in metronidazole and tinidazole SOS activity of NfsA_Pp mutant Pp_G5, (Figure 4.3), growth inhibition assays were performed using both prodrugs (Figure 4.5A). Identical growth inhibition assays were performed using SOSR4 cells expressing wild type NfsA_Pp to enable comparison (Figure 4.5B). Unfortunately, time constraints prevented the measurement of accurate metronidazole and tinidazole $\mathrm{IC}_{50}$ values. The results still demonstrated, however, that while the metronidazole activity had decreased compared to wild type NfsA_Pp, so had its tinidazole activity, to the point where the mutant was not noticeably more tinidazole selective than wild type NfsA_Pp. Addition evolution may therefore be necessary before the tinidazole selectivity of this NfsA_Pp mutant can be improved. 
A)

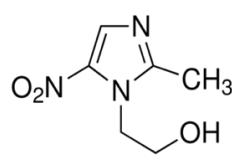

Metronidazole<smiles>CCS(=O)(=O)CCn1c([N+](=O)[O-])cnc1C</smiles>

Tinidazole

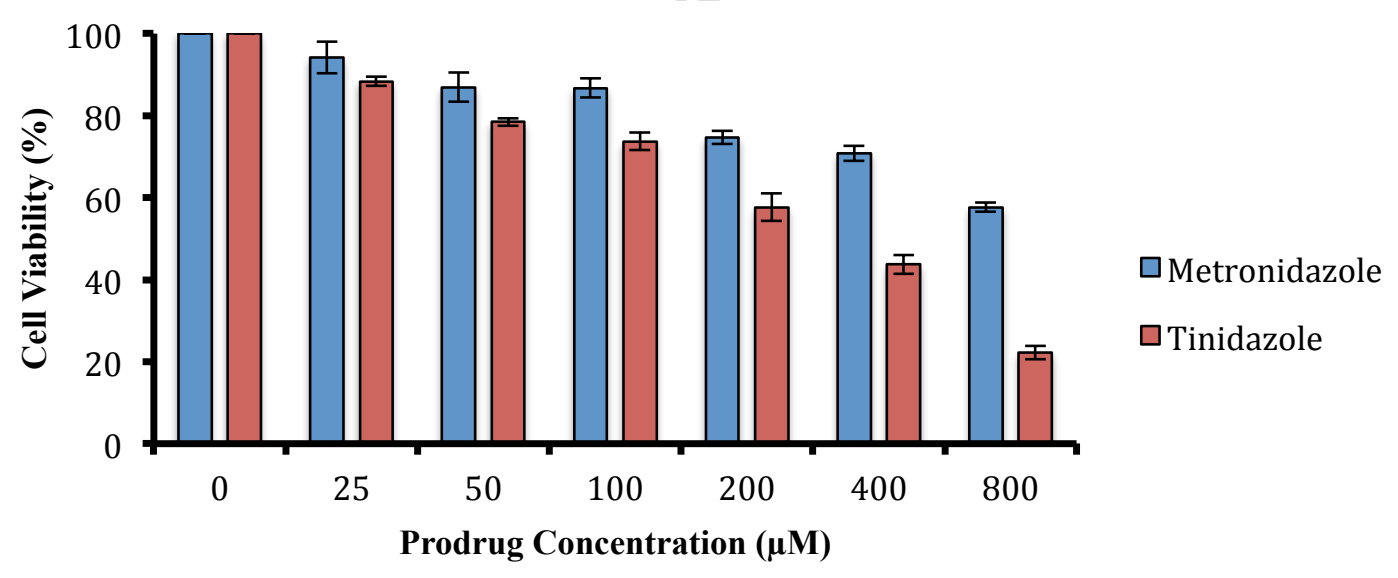

B)

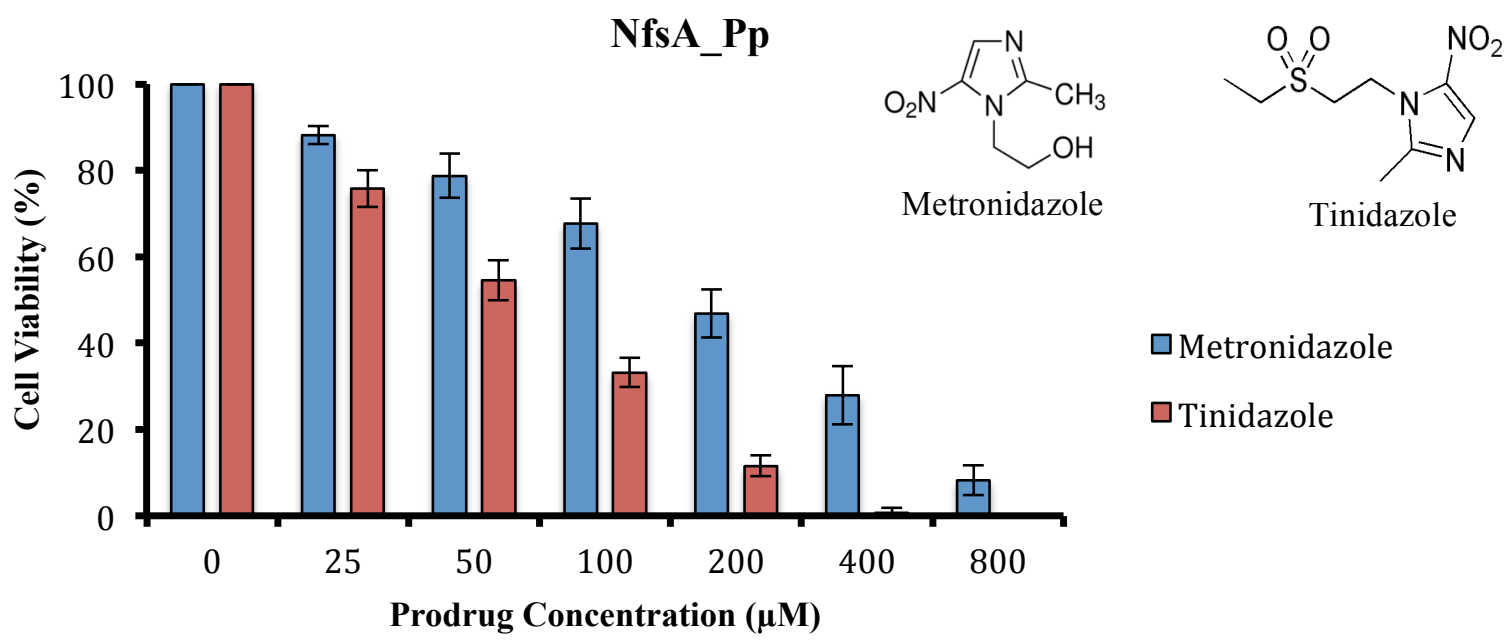

Figure 4.5 - Metronidazole and tinidazole growth inhibition assays of $E$. coli SOS-R4 overexpressing either NfsA_Pp or mutated NfsA_Pp enzyme. Cultures of E. coli SOS-R4 overexpressing either A) NfsA_Pp mutant Pp_G5 or B) Wild NfsA_Pp from pUCX were challenged in 384 well plates with separate serial dilutions of metronidazole and tinidazole for $4 \mathrm{~h}$. Growth was measured as the difference in culture turbidity $\left(\mathrm{OD}_{600}\right)$ between pre $(\mathrm{t}=0 \mathrm{~h})$ and post $(\mathrm{t}=4 \mathrm{~h})$ drug challenged cultures. For each strain, growth inhibition was measured as the percentage decrease between challenged cultures and its respective unchallenged control. For each prodrug, data are representative of three independent repeats \pm SEM. 


\subsection{Chapter 4 Discussion}

The goal of the research described in this chapter was to improve the tinidazole selectivity of NfsA_Pp using directed evolution. This work was intended as a parallel strategy to the evolution of NfsA_Ec described in the previous chapter. The goal of both strategies was to enhance the tinidazole selectivity of NfsA enzymes to complement the highly metronidazole selective $\mathrm{NfsB}_{-} \mathrm{Vv}$ in a multiplex ablation system. Owing to the early success of the NfsA_Ec 7SM strategy, and the fact that NfsA_Pp is less active overall with tinidazole, greater focus was given to the former work than to epPCR mutagenesis of NfsA_Pp.

Nonetheless, it was demonstrated that niclosamide was again an effective positive selection agent for selecting for active nitroreductase mutants, in this case NfsA_Pp mutants generated using error prone PCR, that were active with metronidazole and tinidazole (Figure 4.2). In contrast to the 7SM NfsA_Ec mutants in Figure 3.8, none of the NfsA_Pp mutants selected using niclosamide were considerably more metronidazole or tinidazole active than wild type NfsA_Pp. The reason for this difference is likely due to the NfsA_Pp mutants containing far few active site mutations (average of 4-5 DNA mutations in total, randomly dispersed across the full length of each gene) than the NfsA_Ec 7SM library (seven targeted codon changes per gene, each in or near the active site), making radical changes to enzyme activity less likely.

As observed with the NfsA_Ec 7SM library in Figure 3.14, negative selection using metronidazole selected against NfsA_Pp mutants possessing wild type levels of metronidazole activity (Figure 4.3). While many of the mutants had retained comparable higher levels of tinidazole activity, none were as obviously as tinidazole selective as the NfsA_Ec 7SM mutants evolved in Chapter 3. The one NfsA_Pp mutant sequenced, Pp_G5, contained two amino acid changes (Table 4.2). A more rigorous analysis, such modeling the Pp_G5 amino acid sequence against the NfsA_Ec crystal structure, or a crystal structure of Pp_G5 itself would be required to draw conclusions about the catalytic role of either of the amino acid changes present.

Metronidazole and tinidazole growth inhibition analysis of NfsA_Pp mutant Pp_G5, also demonstrated it was not notably more tinidazole selective than wild type NfsA_Pp, rather 
demonstrated similarly reduced activity with both prodrugs. Calculating $\mathrm{IC}_{50}$ values would be beneficial in the future to better characterise the changes in metronidazole and tinidazole selectivity of this mutant. Collectively, these findings are consistent with the observation that the "smart library" approach of targeting key residues using saturation mutagenesis codons (as done to create the NfsA_Ec 7SM library) can often yield superior outputs faster than a purely random mutagenesis approach (Chica et al., 2005). Identifying and targeting key NfsA_Pp catalytic amino acid codons for saturation mutagenesis, as done for NfsA_Ec, could therefore be a promising approach for improving tinidazole substrate selectivity in the future.

The NfsA_Ec single mutant assays performed in Section 3.2.9 indicated that single amino acid changes alone, even those or near the active site, were unable to enhance tinidazole selectively. While NfsA_Pp mutant Pp_G5 contained two amino acid changes, these were still insufficient for a substantial improvement in tinidazole selectivity. Additional rounds of mutagenesis and selection are therefore likely required before improvement in tinidazole selectivity will be possible. Furthermore, identifying the minimum set of amino acid changes required for improving the tinidazole selectivity of the NfsA_Ec 7SM mutants in Chapter 3 could assist with the evolution of NfsA_Pp. For instance, once identified, the codons of the equivalent amino acids in NfsA_Pp could be targeted for site-saturation mutagenesis.

Like NfsA_Ec, the native NfsA_Pp enzyme is also more selective for the green profluorophores FSL76 and FSL150 than the red profluorophore FSL178 (Condon, 2013; Horvat, 2012). In previous validation studies, a mixed population HEK293 cells expressing either NfsA_Pp or NfsB_Vv were capable of being differentiated by coculturing with FSL76 and FSL178, respectively (Condon, 2013). As observed in Section 3.2.10, however, evolving NfsA_Ec to be more tinidazole selective was highly detrimental to its activity with FSL76 and FSL150. It will therefore be important to measure the FSL76/FSL150 activity of any promising tinidazole selective NfsA_Pp mutants evolved in future to determine their multiplex imaging potential. 


\section{Chapter 5: Metagenomic Discovery of New Nitroreductases}

The goal of this chapter was to discover new nitroreductase enzymes from metagenomic libraries and assess their potential for targeted cell ablation. Rather than focus on the genomes of culturable bacteria, as had been done previously (Prosser et al., 2013), we chose to focus on the genomes of unculturable bacteria, a previously neglected source. Mounting evidence suggests that culturable bacteria make up a very small minority of those present in the environment (Stewart, 2012; Torsvik et al., 2002). The genomes of unculturable bacteria, although more difficult to study, are therefore a potentially rich resource for new nitroreductase enzymes. As discussed in Section 1.3.2, studying the genes and gene products of unculturable microorganisms requires methods that circumvent the need to culture them directly. Creating and screening metagenomic libraries, created from environmental DNA (eDNA) samples, in heterologous hosts is the primary method to achieve this.

The two main metagenomic screening strategies are functional and homology-based screens. Functional screening techniques detect target eDNA genes on the basis of a detectable phenotypic change associated with their expression (Piel, 2011). The alternative to functional screening, homology-based screening, in contrast, detects target genes within metagenomic libraries on the basis of their homology to known members of the same gene family. A limitation of homology screens is that, due to their dependence on sequence similarity, they are restricted to finding new members of already characterised gene families, whereas functional screens can potentially discover entirely new enzyme families (Piel, 2011). To avoid expanding our collection of nitroreductases according to preconceived biases as to which were generally the most active i.e. the NfsA and NfsB family enzymes, we therefore chose to focus on the development of functional metagenomic screens for nitroreductase enzymes.

Function based metagenomic screening and directed evolution actually parallel each other in a number of ways. Both involve screening libraries of mostly useless stretches of DNA trying to find the few that possess a desired activity. Furthermore, for either process to be possible, both require methods of detecting the desired enzymatic activity. It is therefore unsurprising that directed evolution methods and function-based 
metagenomic techniques can overlap to some degree, a point that will be expanded later in this chapter.

Two different metagenomic libraries were available for screening in this research. The first, referred to as the Swedish library, was created from the DNA extracted from a sample of garden compost (Parachin and Gorwa-Grauslund, 2011). The Swedish library contained approximately $1.26 \times 10^{5}$ eDNA fragments with an average size of approximately $4 \mathrm{~kb}$, each present within the plasmid pRSETB (Parachin and GorwaGrauslund, 2011). The other metagenomic library available, referred to as the New Zealand (NZ) library, was created by the Ackerley lab from a sample of garden soil and present in pETDUET plasmids (Owen et al., 2012). Both libraries had been transformed into E. coli host cells and successfully screened to identify novel PPTase enzymes and their associated biosynthetic gene clusters (Owen et al., 2012).

\subsection{Metagenomic Screening using Profluorophore Molecules}

The first functional screening technique attempted utilized nitroaromatic profluorophores to detect the expression of nitroreductases from eDNA inserts. As demonstrated in Section 3.2.10, the activation of a profluorophore by a nitroreductase can result in the emission of visible light. We hypothesised that profluorophore activation could used to detect the expression of a nitroreductase within an eDNA insert. To enable high throughput screening, we envisaged incubating E. coli cells containing metagenomic libraries to a profluorophore prior to collecting the most fluorescent cells i.e. those expressing a nitroreductase and thus capable of activating the fluorophore, using FACS.

Of the 54 different profluorophore molecules available in the ACSRC library, the profluorophore FSL41 (structure presented in Figure 3.24) was selected. FSL41 was chosen because it had previously been validated as a means of detecting E. coli cells overexpressing a nitroreductase enzyme in a flow cytometer (Horvat, 2012). Additionally, unlike most other profluorophores in the ACSRC library, a range of different nitroreductase enzymes were active with FSL41, increasing the chances of detecting activation by an eDNA nitroreductase (Horvat, 2012). Moreover FSL41 remained cell entrapped once activated (Horvat, 2012), which we believed could prevent 
activated profluorophore diffusing into neighboring, non-fluorescent cells, potentially resulting in the FACS machine falsely identifying them as positive events.

\subsubsection{Activation of FSL41 by $E$. coli W3110 Cells}

Prior to library screening, the FACS approach was validated and optimized. While the ability of overexpressed nitroreductase enzymes to activate profluorophores was well established (Condon, 2013; Horvat, 2012), it was unknown whether lower levels of nitroreductase expression, such as might be expected from a metagenomic insert, were also detectable. Expression levels of eDNA can often be low due to the heterologous host (E. coli in this case) being inefficient in their ability to express the foreign DNA (Gabor et al., 2004).

Lacking a pre-recovered nitroreductase within a large eDNA insert, which would be the ideal positive control for this work, we instead chose to test if wild type E. coli (W3110) cells could activate FSL41. While W3110 cells expresses at least two enzymes known to be active with FSL41, NfsA_Ec and NfsB_Ec (Horvat, 2012; Prosser et al., 2013), here both of these enzymes would be expressed at physiological levels, rather than overexpressed from a pUCX plasmid. We reasoned that if W3110 cells were unable to noticeably activate FSL41, there would be little chance that single nitroreductase expressed from a metagenomic insert could either. As a negative control, the nitroreductase knockout strain E. coli 7TL, used for the expression of metagenomic libraries (discussed in the following section), was used. Results from the FSL41 assay comparing FSL41 fluorescence of W3110 cells to E. coli 7TL cells are displayed in Figure 5.1. W3110 cells incubated with $10 \mu \mathrm{M}$ FSL41 were seven fold more fluorescent than those exposed only to the DMSO vehicle. In contrast, E. coli 7TL cells incubated with $10 \mu \mathrm{M}$ FSL41 were no more fluorescent than the same cells incubated with an equivalent volume of DMSO. Collectively, these results demonstrated that nitroreductase overexpression was not required for the detectable activation of FSL41, proving reassurance that the detection of nitroreductases expressed from eDNA inserts could indeed be possible. Furthermore, this experiment demonstrated the benefit of using the $E$. coli 7TL strain as the heterologous host strain. As E. coli 7TL cells lacked the background level of FSL41 activation observed in W3110 cells, low levels of 
profluorophore activation by an eDNA nitroreductase could potentially be more pronounced, i.e. less likely to be swamped by endogenous FSL41 activation.

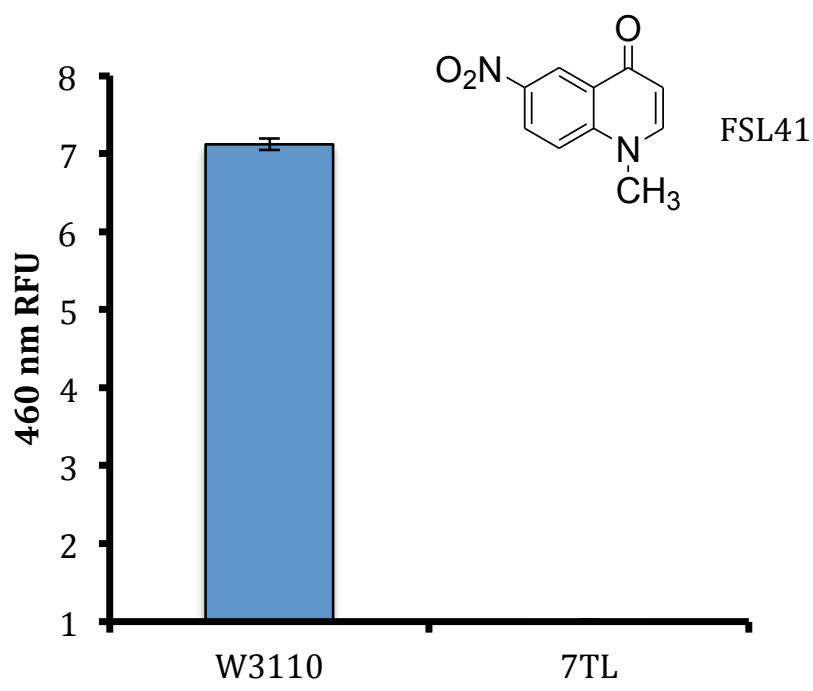

Figure 5.1 - FSL41 fluorescence assay on W3110 and $E$. coli 7TL cells. Mid-exponential phase cultures of W3110 and $E$. coli 7TL cells were exposed to $10 \mu \mathrm{M}$ FSL41, or to an equivalent amount of DMSO, for $1 \mathrm{~h}$. Fluorescence of the activated (nitro-reduced) FSL41 (ex 355/em $460 \mathrm{~nm}$ ) and culture turbidity $\left(\mathrm{OD}_{600}\right)$ were then measured. The raw blue fluorescence values of each culture were normalized by their respective $\mathrm{OD}_{600}$ values. $460 \mathrm{~nm}$ relative fluorescence units (RFU) were then calculated by the fold difference in fluorescence between each culture exposed to FSL41 and its respective DMSO control. Data are representative of three independent experiments \pm SEM.

\subsubsection{Increased Expression of pETDUET Plasmids in the E. coli 7TL Strain}

In addition to lacking background levels of FSL41 activation, E. coli 7TL strain was also selected because, unlike the other available nitroreductase knockout strains, it contained an IPTG-inducible T7 RNA polymerase gene. As the eDNA fragments of both the Swedish and New Zealand had been cloned adjacent to a T7 promoter sequences in their respective plasmid vectors (Owen et al., 2012; Parachin and Gorwa-Grauslund, 2011), the presence of T7 RNA polymerase could increase the level of eDNA gene expression, thereby increasing the chances of detection (Ahmad et al., 2011; Gabor et al., 2004). A proof-of-concept experiment was performed to demonstrate the higher levels of pETDUET (the vector the NZ library was cloned into) expression in E. coli 7TL cells relative to a control strain lacking T7 RNA polymerase. To model a nitroreductase within 
a pETDUET eDNA insert, the nitroreductase NfsB_Ec gene was cloned into a pETDUET plasmid. While a nitroreductase within an eDNA insert was unlikely to be immediately adjacent to the $\mathrm{T} 7$ promoter, provided it was not separated by a transcriptional terminator sequence then T7 promoter-driven expression might still be possible (Gabor et al., 2004). pETDUET_NfsB was then transformed into either E. coli 7TL cells, or E. coli 7NT cells (the latter being identical to $E$. coli 7TL in terms of chromosomal nitroreductase deletions, but lacking T7 RNA polymerase). Expression of NfsB_Ec, as determined by SDS-PAGE, was only detected in E. coli 7TL cells containing a pETDUET_NfsB_Ec plasmid when T7 RNA polymerase expression had been induced (Figure 5.2). From these data we concluded that the E. coli 7TL strain was capable of increasing expression of nitroreductase genes adjacent to the $\mathrm{T} 7$ promoter of pETDUET. Using it as the host strain could therefore increase the chances of expressing an eDNA nitroreductase at levels detected by the activation of FSL41. 


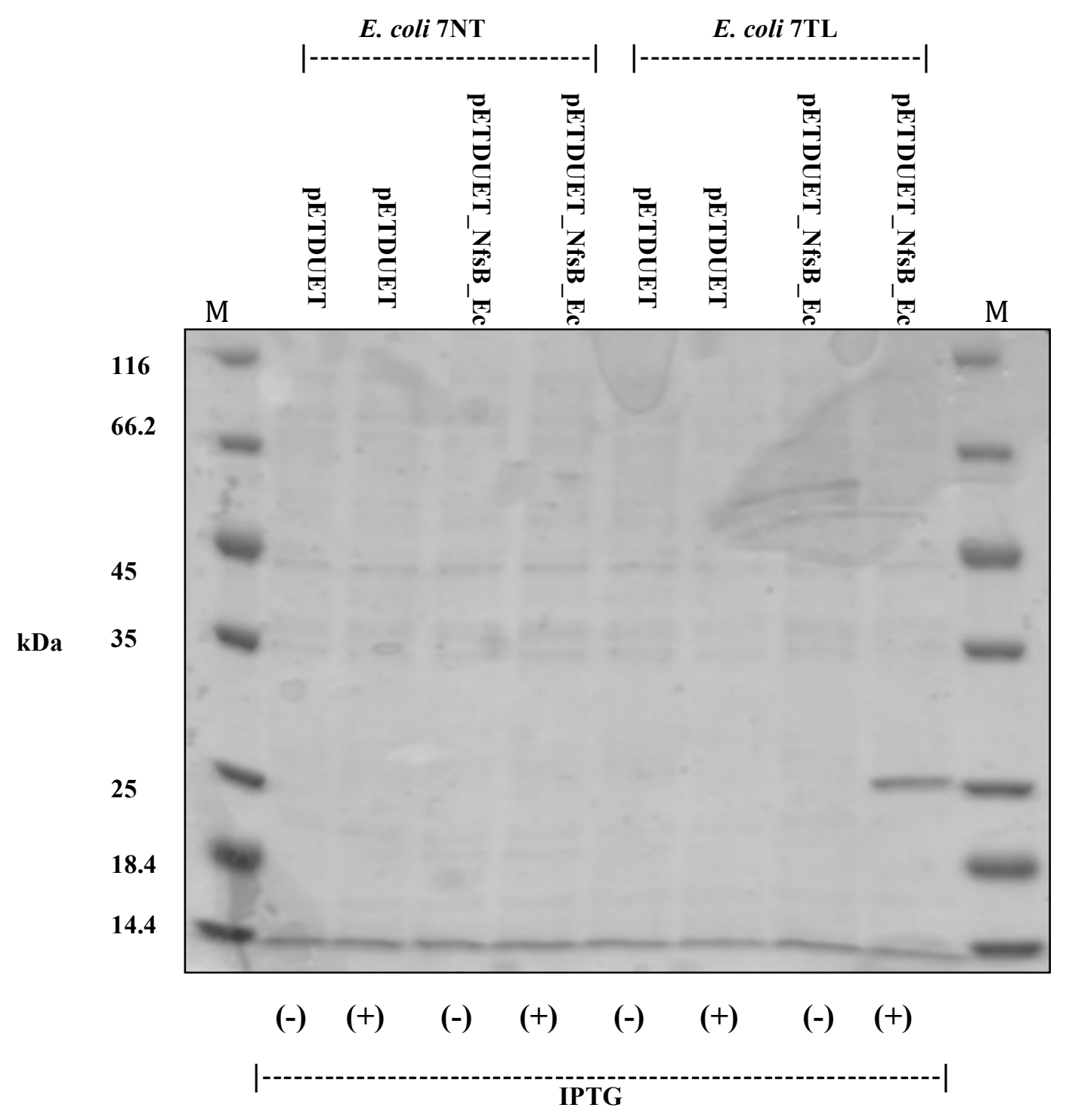

Figure 5.2 - SDS-PAGE gel demonstrating induced expression of pETDUET_NfsB_Ec from E. coli 7TL cells. Overnight cultures of each strain (either E. coli $7 T L$ containing a T7 RNA polymerase gene, or E. coli 7NT lacking a T7 RNA polymerase gene) were inoculated into fresh media in duplicate and grown to an OD600 of 0.5. IPTG was then added to one of the duplicates to a final concentration of $0.5 \mathrm{mM}$ before all cell cultures were grown for an additional 4 hours. Cell cultures were normalized by $\mathrm{OD}_{600}$ prior to loading into a $15 \%$ SDS-PAGE cell to visualise protein expression. $\mathrm{M}=$ protein size standard. NfsB_Ec $=25.7 \mathrm{kDa}$ in size. 


\subsubsection{Proof of Concept FACS Enrichment of Nitroreductase Overexpressing Cells}

For FACS to be an effective metagenomic screening strategy, it was necessary that it detect and sort fluorescent nitroreductase expressing cells against the rest of the larger, non-fluorescent, metagenomic library. To model this, E. coli 7TL cells containing pETDUET_NfsB_Ec were diluted into a culture of E. coli 7TL cells containing an empty pETDUET plasmid at a 1:999 ratio. The mixed culture was incubated with $50 \mu \mathrm{M}$ FSL41, the concentration previously found to be successful in detecting FSL41 activation via flow cytometry using a $488 \mathrm{~nm}$ laser and a 450/50 bandpass filter (Horvat, 2012). An identical population of cells that had not been exposed to FSL41 was run though the flow cytometer first to establish background levels of fluorescence. The mixed 1:999 cells population that had been incubated with FSL41 was then run through the FACS machine. Although exact proportions were unfortunately not recorded, only approximately $1 \%$ of these cells appeared more fluorescent than the cells that had not been incubated with FSL41. The top $10 \%$ of this fluorescent population was collected (10,000 events in total). For purposes of comparison, the bottom $10 \%$ of cells in the whole population that gave the least fluorescence signal was also sorted (also 10,000 events in total). Each sorted cell population was then plated on LB agar to obtain single colonies. From population, 114 individual colonies were picked and exposed to FSL41 to determine if FACS had successfully enriched for the fluorescent $E$. coli 7TL cells expressing pETDUET_NfsB_Ec. A representative subset (57) of the colonies of each population screened using FSL41 is displayed in Figure 5.3. 


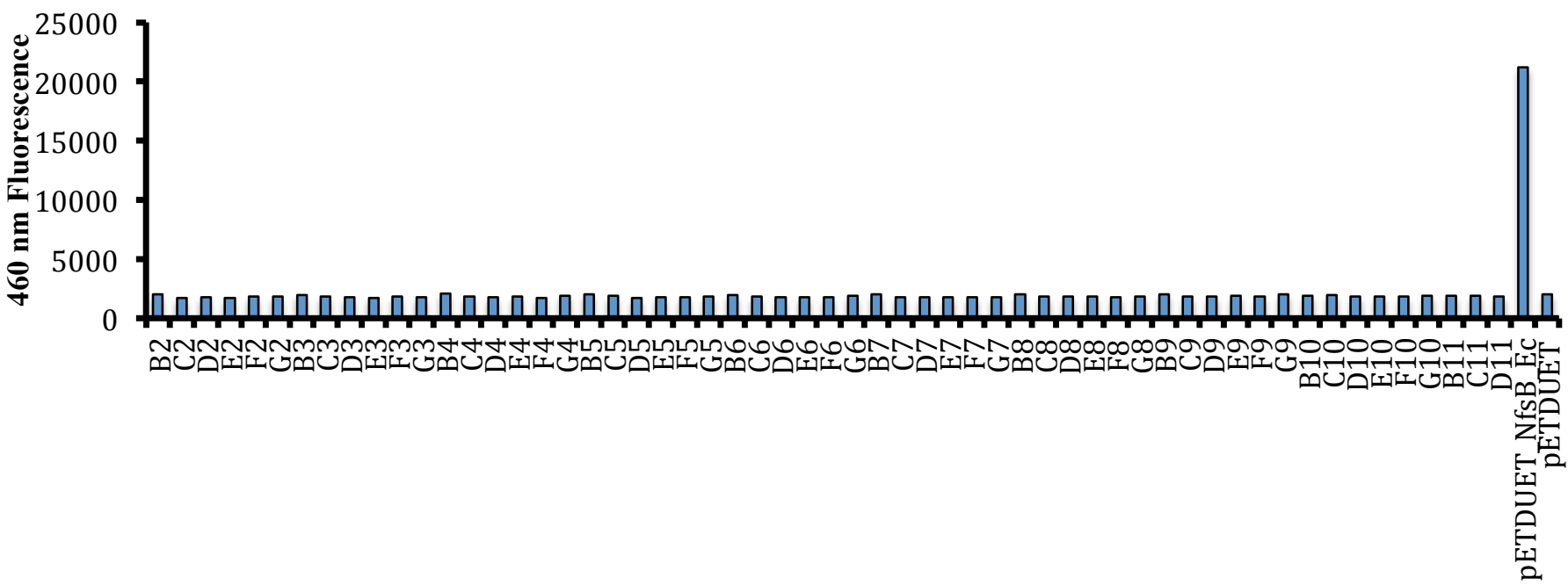

B) Bottom $10 \%$ of Least Fluorescent Cells Sorted using FACS<smiles>Cn1ccc(=O)c2cc([N+](=O)[O-])ccc21</smiles>

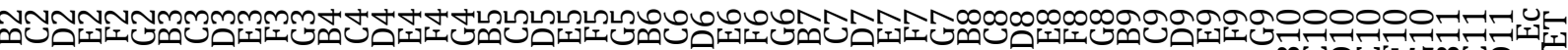

Figure 5.3 - FSL41 fluorescence assay of post-FACS population of $E$. coli 7TL pETDUET_NfsB_Ec cells diluted into a culture of $E$. coli 7TL pETDUET empty cells at a 1:999 ratio. After being normalized for culture turbidity $\left(\mathrm{OD}_{600}\right)$, mid-exponential phase E. coli 7TL pETDUET_NfsB_Ec cells were diluted into mid-exponential phase $E$. coli 7TL pETDUET cells at a 1:999 ratio. The cell mixture was then incubated with $50 \mu \mathrm{M}$ FSL41 for $1 \mathrm{~h}$ before being run through a FACS machine using a $488 \mathrm{~nm}$ laser and a $450 / 50$ bandpass filter. The top $10 \%$ of the subset of fluorescent cells (this subset being approximately $1 \%$ of the total pool) were collected and the bottom $10 \%$ of least fluorescent cells in the whole cell population were collected. Each of the sorted populations was then plated on LB agar to form single colonies. From each sorted population, 57 random colonies were picked and grown as liquid cultures from which to run $10 \mu \mathrm{M}$ FSL41 fluorescence assays. After $1 \mathrm{~h}$ incubation period with $10 \mu \mathrm{M}$ FSL41, blue fluorescence was recorded (em $420 \mathrm{~nm} / \mathrm{ex} 460 \mathrm{~nm}$ ). A) FSL41 fluorescence assays run on single colonies collected by FACS sorting the top $10 \%$ of the subset of fluorescent events in the mixed population B) FSL41 fluorescence assays run on single colonies collected by FACS sorting the bottom 10\% of the least fluorescent events in the whole mixed population. E. coli 7TL cells expressing pETDUET_NfsB_Ec was included as a positive fluorescence control. E. coli 7TL cells containing an empty pETDUET plasmid were included as a negative fluorescence control. Data are representative of one experiment. 
The colonies randomly screened from each of the sorted populations resembled each other exactly in the FSL41 fluorescence assays. None of the colonies screened from either population were more fluorescent than the empty pETDUET negative control, indicating they did not contain a pETDUET_NfsB_Ec plasmid. The pETDUET_NfsB_Ec positive control was highly distinguishable by its activation of FSL41, demonstrating that had FACS enriched for fluorescent pETDUET_NfsB_Ec cells, they would have been readily detectable. It was therefore concluded that FACS was unable to enrich for fluorescent pETDUET_NfsB_Ec cells when diluted at a 1:999 ratio in E. coli 7TL empty pETDUET cells. During the FSL41 optimization experiments performed prior to FACS, no difference in culture turbidity $\left(\mathrm{OD}_{600}\right)$ was observed between E. coli 7TL expressing pETDUET_NfsB_Ec incubated with $50 \mu \mathrm{M}$ FSL41 for 1 $\mathrm{h}$ (the pre-FACS incubation time), verses the same cells incubated with an equivalent volume of DMSO. This result indicated that toxicity of FSL41 to E. coli 7TL expressing pETDUET_NfsB_Ec resulting in cell death was unlikely to be the reason for the lack of enrichment.

Given that the average insert size of the Swedish and NZ libraries was $\sim 4-7 \mathrm{~kb}$, and the average bacterial gene length is $\sim 1 \mathrm{~kb}$, several genes could be present per eDNA insert. Screening 1000 eDNA clones could therefore represent around 3000-4000 intact eDNA genes. However, only $40 \%$ of these would be likely to express in E. coli (Gabor et al., 2004), and only a subset of encoded nitroreductase enzymes are likely to be active with FSL41. Making the conservative estimates of an average environmental bacterial genome size of $5 \mathrm{Mbp}$ (slightly larger than E. coli (Blattner et al., 1997)) and only one or two FSL41-active nitroreductase per genome, it can reasonably be assumed that detectable eDNA nitroreductase enzymes would be present in our libraries at lower ratio than 1:999. Furthermore, those nitroreductase enzymes are very likely to be expressed at lower levels than pETDUET_NfsB_Ec. From these data we concluded that FACS would be unlikely to enrich for $E$. coli 7TL cells containing eDNA nitroreductases. Rather than continue to devote time and resources into optimizing the FACS procedure, we decided to try alternative metagenomic screening strategies. The first of which explored the use of niclosamide as a positive selection for nitroreductases expressed within eDNA inserts. 


\subsection{Niclosamide Metagenomic Screening}

As the directed evolution experiments Chapter 3 and Chapter 4 demonstrated, niclosamide could select for $E$. coli clones expressing active nitroreductases, inhibiting the growth of those lacking nitroreductase expression (provided they also lack the TolC exporter). From these data, we tested if plating a metagenomic library on LB agar supplemented with niclosamide, selected for metagenomic clones expressing eDNA nitroreductases.

Using niclosamide as a metagenomic screening strategy had actually previously been tested in a one-off experiment by Dr. Janine Copp and myself (unpublished). When we attempted this strategy, of the twenty sequenced E. coli 7TL clones identified as viable on niclosamide, all were found to express eDNA cellular efflux transporters, complementing the deleted tolC gene. Reasoning that nitroreductases might be being recovered at low levels in amongst a preponderance of tolC genes, we sought to introduce a secondary screening step to identify any metagenomic clones expressing nitroreductase enzymes. We tested whether the profluorophore FSL41 could differentiate clones expressing eDNA nitroreductase enzymes from those expressing eDNA efflux pumps. To test this, E. coli 7TL cells transformed with the Swedish or NZ Library were plated on LB agar containing $0.5 \mu \mathrm{M}$ niclosamide. While multiple niclosamide concentrations were tested, to allow for the possibility that due to lower expression levels eDNA nitroreductases might be less capable to detoxify niclosamide (than a nitroreductase overexpressed in pUCX), $0.5 \mu \mathrm{M}$ was ultimately selected again because it reliably inhibited the growth of E. coli 7TL negative control cells.

After colony formation had occurred, 228 colonies of the Swedish library and 228 colonies of the NZ library were grown as liquid cultures to perform FSL41 fluorescence assays. In the results of these assays, none of the niclosamide-selected eDNA clones from either library were any more fluorescent than background levels. It was therefore concluded that the vast majority of metagenomic clones viable on niclosamide were not nitroreductase enzymes, but more likely cellular efflux pumps as previously seen (Janine Copp, Rory Little, unpublished). These data suggested that efflux pumps conferred a more substantial resistance to niclosamide than nitroreductases did, such that even very low-level expression of an efflux pump enabled a transformed E. coli 7TL cell to form a 
colony, whereas high-level expression of a nitroreductase might be required for the same effect.

While we still believed niclosamide could select for eDNA nitroreductases if they were expressed at high enough levels, a limiting factor in their identification could have been the relatively low throughput nature of the FSL41 counter-screen. If niclosamideresistant metagenomic clones could be counter-screened in a higher throughput manner, then a clone expressing a nitroreductase enzyme would have a greater chance of being identified. We therefore directed our focus toward developing high throughput colonylevel screens to detect nitroreductase expression.

\subsection{SOS/ß-Galactosidase Metagenomic Screening}

The first high-throughput nitroreductase detection screen we explored involved use of the DNA-damage inducible genomic lacZ construct of the E. coli SOS-R2 strain. Prior to creation of the SOS-R4 GFP strain (Copp et al., 2014), the Ackerley lab used induction of $\beta$-galactosidase from a lacZ gene under the control of an SOS promoter (pSOS_lacZ) as their primary method to measure the DNA damage induced by an activated prodrug (Prosser et al., 2010; Swe et al., 2012). In these assays, $\beta$-galactosidase expression was quantified using the colourimetric lactose analogue, ONPG, which was converted into a yellow product by $\beta$-galactosidase metabolism. However, $\beta$-galactosidase can also metabolize the lactose analogue, X-gal, to produce a blue pigment. The metabolism of Xgal by $\beta$-galactosidase is commonly used in the pUC family of cloning vectors for detecting the insertional disruption of $l a c Z$, indicating a successful ligation event in blue/white colony screening.

Given that X-gal metabolism was a well-established marker for colonies expressing $\beta$ galactosidase, and that the SOS-R2 strain expressed $\beta$-galactosidase in response to DNA damage, we sought to combine the two for the detection of metagenomic clones that expressed nitroreductase enzymes. Our rationale was that, in the presence of a prodrug, a metagenomic clone expressing a nitroreductase should induce the expression of $\beta$ galactosidase from the SOS promoter, whereas clones lacking nitroreductase expression should not. Staining the colonies using X-gal could then be used to identify the 
nitroreductase expressing colonies by turning them blue. If successful, this strategy would increase the throughput of library screening, as colonies would not need to be picked and grown as liquid cultures (as was required with profluorophore metagenomic screening (Section 5.1.3). Rather, a single large Petri dish containing thousands of colonies could be screened simultaneously for $\beta$-galactosidase expression.

One concern was that leaky expression of $\beta$-galactosidase from SOS-R2 cells might turn all colonies blue, even those not expressing a nitroreductase, thus preventing the identification of colonies that were actually expressing an eDNA nitroreductase enzyme. To test whether this would be the case, SOS-R2 cells lacking nitroreductase expression (containing an empty pUCX plasmid) were plated on LB agar containing X-gal. After overnight incubation, all colonies had indeed turned blue, confirming that the basal level of $\beta$-galactosidase expression from SOS-R2 was sufficient to metabolize X-gal.

We next tested whether colonies expressing nitroreductase would turn blue faster than colonies lacking nitroreductase expression if exposed to a prodrug and X-gal simultaneously. LB agar plates containing colonies of either SOS-R2 cells expressing NfsA_Ec from a pUCX plasmid, or SOS-R2 cells containing an empty pUCX plasmid were exposed to $270 \mu \mathrm{g} \cdot \mathrm{mL}^{-1} \mathrm{X}$-gal and $5 \mu \mathrm{M}$ metronidazole in a molten top agarose solution. It was observed that colour change occurred very rapidly for both strains, and after 20 minutes both the pUCX $n f S A_{-} E c$ and pUCX empty plasmid colonies had turned an indistinguishable blue. Overall these results suggested that using SOS/ $\beta$-galactosidase for metagenomic screening would be unlikely to succeed. Even when overexpressed from a pUCX plasmid, NfsA_Ec was not distinguishable from an empty pUCX plasmid control in the presence of the prodrug metronidazole. As a nitroreductase present in a metagenomic insert would to be expressed at lower levels, its detection would be even more unlikely. Although unsuccessful, the central premise of the SOS/ $\beta$-galactosidase detection system, the rapid detection of nitroreductase expression at the level of a colony, inspired the work performed in the next section using the nitroaromatic compound 4nitroimidaoze. 


\subsection{Metagenomic Screening Using 4-Nitroimidazole}

\subsubsection{4-Nitroimidazole can Detect the Expression of Nitroreductase Enzymes at a Colony level}

The final metagenomic screening technique tested utilized the compound 4nitroimidazole. 4-nitroimidazole (Figure 5.4) is a chemical intermediate used industrially in synthesis of the explosive 1-Methyl-2,4,5-trinitro imidazole (Jadhav et al., 2013).

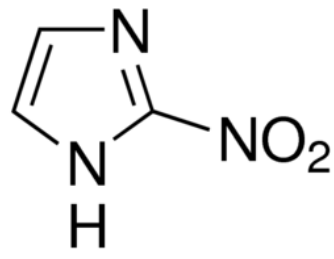

Figure 5.4 - Structure of the prodrug candidate 4-nitroimidazole.

It is also the chemical backbone of several nitro-quenched pET probes of interest to the Ackerley lab for the in vivo imaging of tumour-localised nitroreductase expression. The idea of using 4-nitroimidazole as a metagenomic screening agent originated from a chance observation made by Dr. Elsie Williams, a postdoc in the Ackerley lab. While performing 4-nitroimidazole growth inhibition assays on E. coli expressing NfsA_Ec from pUCX, Dr. Williams noted that liquid cultures containing 4-nitroimidazole turned a dark purple colour. The colour change was not observed, however, in identical cultures of cells containing a pUCX empty plasmid, indicating nitroreductase expression was driving this colour change. While the mechanism behind this colour change was unknown, we hypothesized that it could be used to detect the expression of eDNA nitroreductase genes.

To avoid the cumbersome process of growing individual colonies as liquid cultures, as required for FSL41 fluorescence assays in Section 5.2, we tested whether the colour change of 4-nitroimidazole could used to directly detect nitroreductase expression at a colony level. However, Dr. Williams had also demonstrated that 4-nitroimidazole was converted into a cytotoxin upon nitroreduction (unpublished). Incorporating 4- 
nitroimidazole directly into agar would therefore pose a risk of inhibiting the colony formation of any eDNA nitroreductase clones. To avoid this scenario, we chose to administer 4-nitroimidazole after colonies had already formed, via addition in a molten top agarose solution, as described in Section 5.2. Pilot tests compared colonies of nitroreductase knockout E. coli cells $(7 \mathrm{KO})$ expressing either pUCX NfsA_Ec or containing an empty pUCX plasmid on separate LB agar plates. Molten top agarose supplemented with $5 \mathrm{mM}$ of 4-nitroimidazole was carefully poured over the colonies present on each of the plates. Within 30 minutes at room temperature the NfsA_Ec colonies had turned dark purple (Figure 5.5A), whereas, even after several days, the pUCX colonies had not (Figure 5.5B). From these data we concluded that 4nitroimidazole had great potential to detect single colonies overexpressing a nitroreductase.

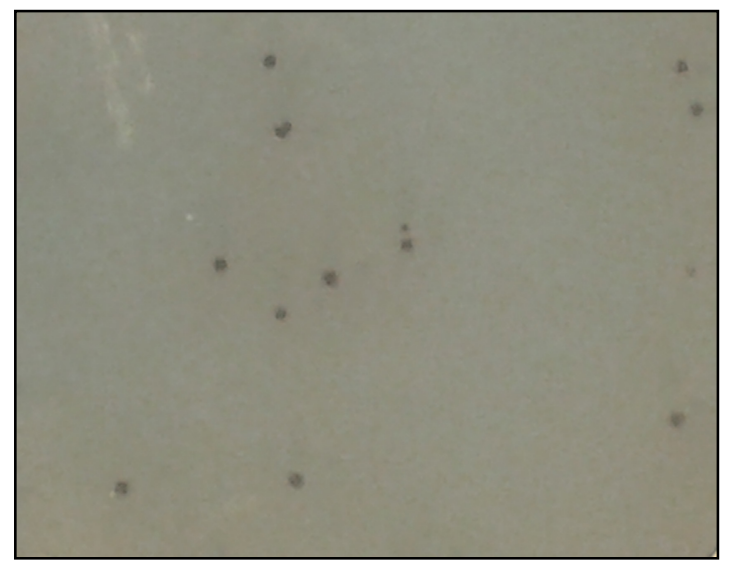

7KO NfsA_Ec

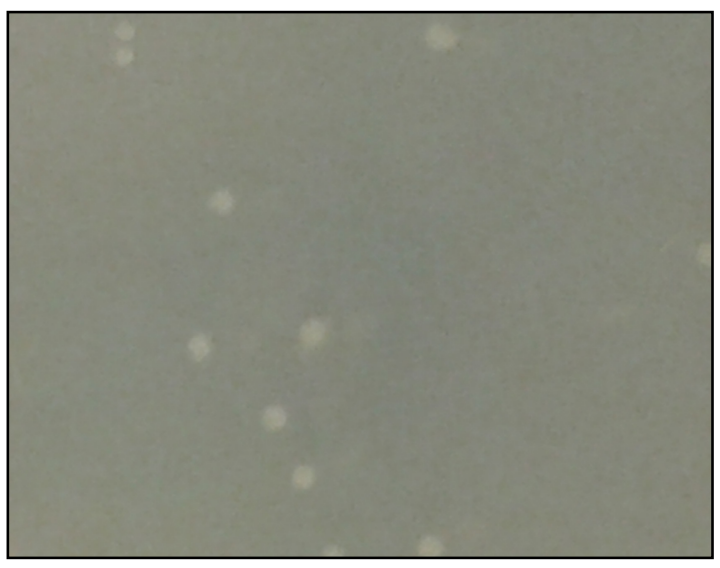

$7 \mathrm{KO}$ pUCX

Figure 5.5 - Reduction of 4-nitroimidazole by nitroreductase overexpressing $E$. coli $7 \mathrm{KO}$ colonies produces a dark purple pigment. E. coli $7 \mathrm{KO}$ cells containing A) pUCX NfsA_Ec, or B) an empty pUCX plasmid, were plated on LB agar to form colonies. After colony formation, molten top agarose containing $5 \mathrm{mM}$ 4-nitroimidazole was poured over the colonies and allowed to cool. Plates were incubated at room temperature for around 30 minutes, over which time the E. coli $7 \mathrm{KO} \mathrm{Nfs}$ _Ec colonies turned a dark purple colour. Photo is representative of all colonies present on each LB agar plate.

A potential concern was whether the reduced form of 4-nitroimidazole might be sufficiently toxic as to prevent subsequent colony recovery in liquid culture. However, individually picking and growing 60 of the E. coli 7KO NfsA_Ec dark purple colonies in 
LB + Amp demonstrated that the recovery of these cells was still possible. The toxicity associated with exposing cells to 4-nitroimidazole using was therefore unlikely to affect recovery and sequencing of any metagenomic clones identified.

We next tested whether lower levels of expression, such as might be expected from an eDNA insert, could also be detected using 4-nitroimidazole. As done previously (Section 5.1.1), we used W3110 cells as a positive control for low-level nitroreductase expression to observe whether the endogenous expression of nitroreductase enzymes from W3110 cells could cause a 4-nitroimidazole colour change. The nitroreductase knockout strain E. coli 7TL cells was used as a negative control in this experiment, as it was the heterologous host used for metagenomic library screening. Previous data had demonstrated that, of the seven endogenous nitroreductases present in W3110, only NfsA_Ec was notably active with 4-nitroimidazole (Condon, 2013). Here, pilot tests (not shown) indicated that 4-nitroimidazole metabolism occurred much more slowly in W3110 cells than in E. coli 7TL cells overexpressing a nitroreductase, resulting in a less pronounced colour change. The translucent growth medium M9 agar was also tested in an attempt to improve the contrast of the coloured colonies, but was ultimately found to make little difference compared to LB agar, and colony formation was much slower on this medium. Greater contrast was observed when the concentration of 4-nitroimidazole was increased to $20 \mathrm{mM}$ and the LB agar plates were incubated at room temperature overnight Figure 5.6. 


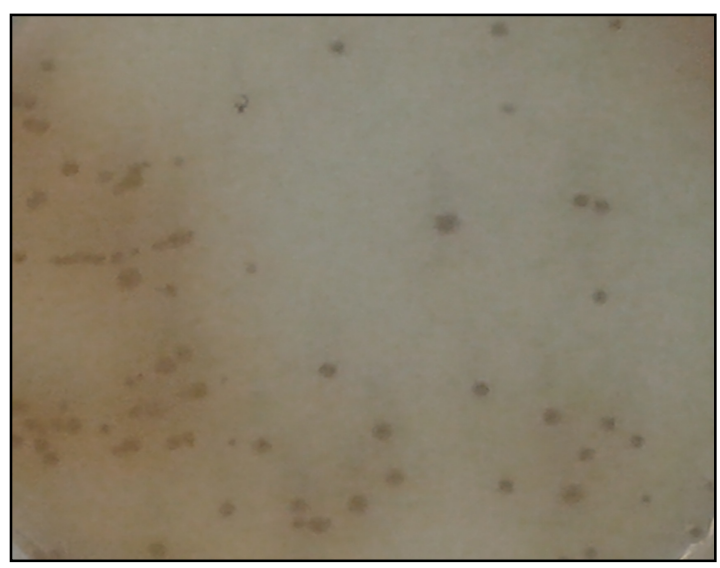

W3110

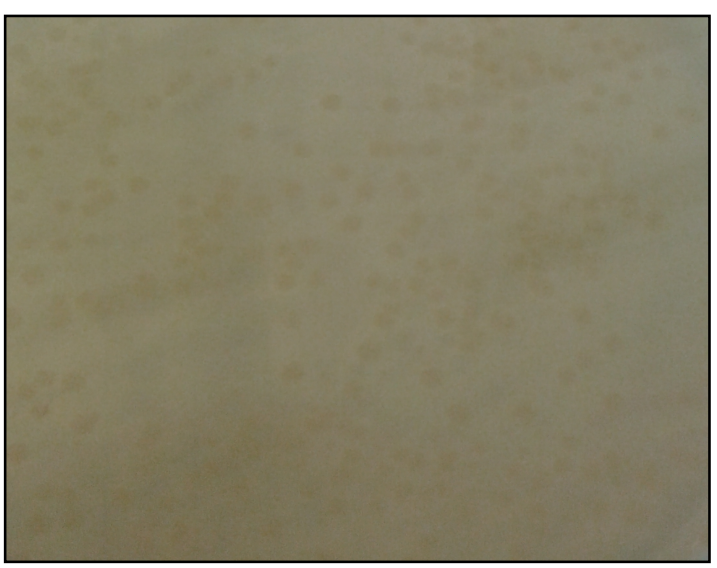

7TL

Figure 5.6 - Reduction of 4-nitroimidazole by W3110 colonies produces a dark purple pigment. A) W3110 cells or B) E. coli 7TL cells (which lacked seven endogenous nitroreductase genes) were plated on LB agar to form colonies. After colony formation, molten top agarose containing $20 \mathrm{mM} 4$-nitroimidazole was poured over the colonies and allowed to cool. Plates were incubated at room temperature overnight, over which time the W3110 colonies turned a dark purple colour. No colour change was observed in E. coli 7TL cells, even after incubation for seven days.

The overexpression of a nitroreductase from a pUCX plasmid was therefore not required for its detection using 4-nitroimidazole. Collectively, these results provided encouragement that lower levels of nitroreductase expression, such as might be present in an eDNA clone, could also be identified by 4-nitroimidazole.

\subsubsection{Successful Detection of W3110 Cells within a Higher Background of $E$. coli 7TL cells}

As discussed in Section 5.1.3 with the likelihood that only a very low proportion of eDNA inserts would contain a nitroreductase gene, it was necessary that 4-nitroimidazole be able to identify these clones against a larger background of insensitive (i.e., pale) clones. To model this scenario, W3110 cells were diluted ten-fold in a culture of E. coli 7TL cells and the cell mixture was plated on LB agar to allow colony formation. Molten top agarose containing $20 \mathrm{mM}$ 4-nitroimazole was then poured over the colonies. Twenty-one colonies that appeared darker than the rest (numbered in Figure 5.7A) were PCR screened to determine if they were W3110 cells, as the colour change suggested. Twenty-one adjacent pale colonies i.e. those that should not be W3110 cells, were also 
PCR screened. $n f S A \_E c$ primers were selected for determining colony identity, as these would amplify the $n f_{S} A$ gene that is present in the genome of W3110, but was absent in $E$. coli 7TL. All twenty-one of the selected dark colonies contained a full-length $n f_{S} A_{-} E c$ gene (Figure 5.7B) indicating they had been correctly identified using 4-nitroimidazole. In contrast, none of the pale colonies selected tested positive for NfsA_Ec (Figure 5.7C). 
A)

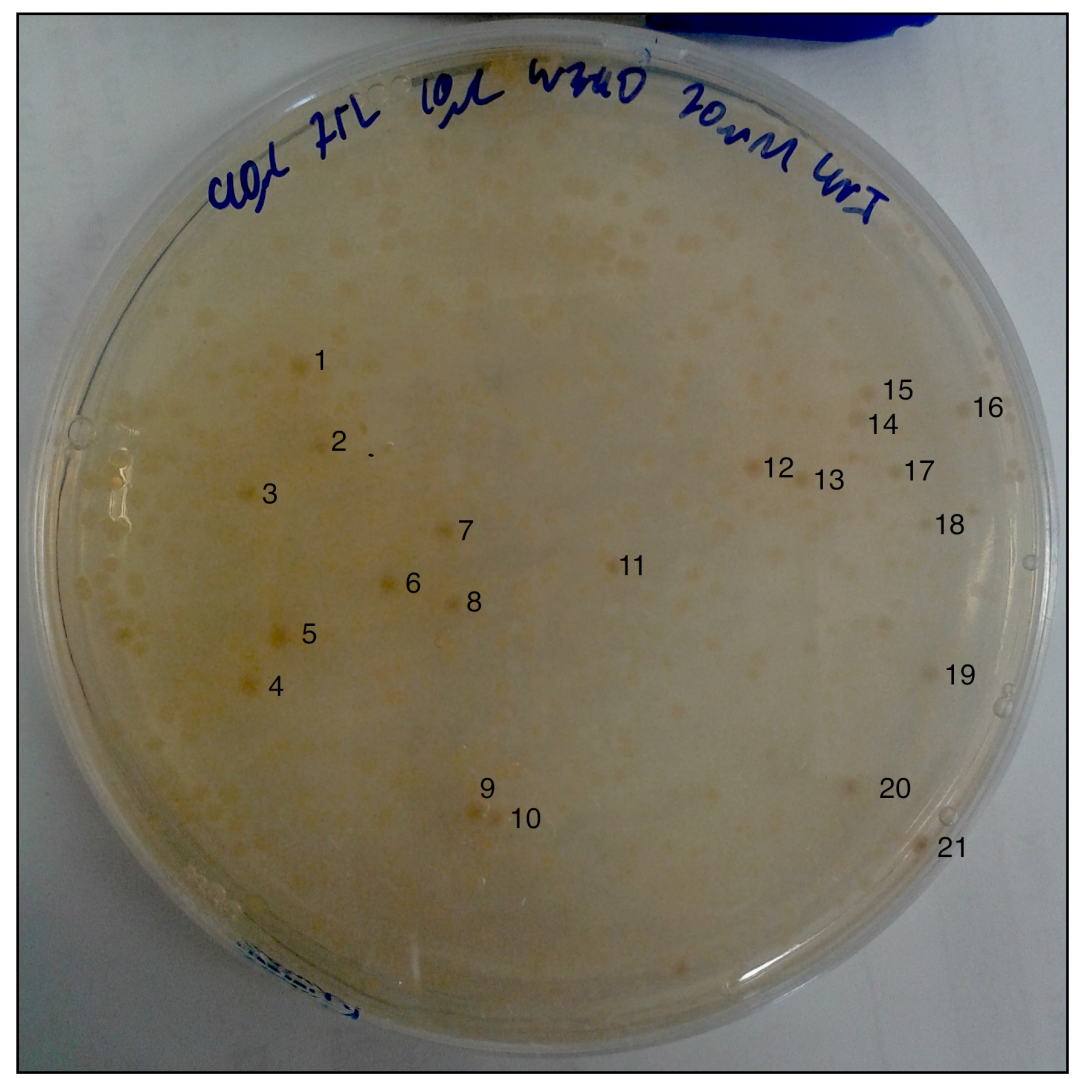

B)

\section{Dark colonies numbered in picture above}

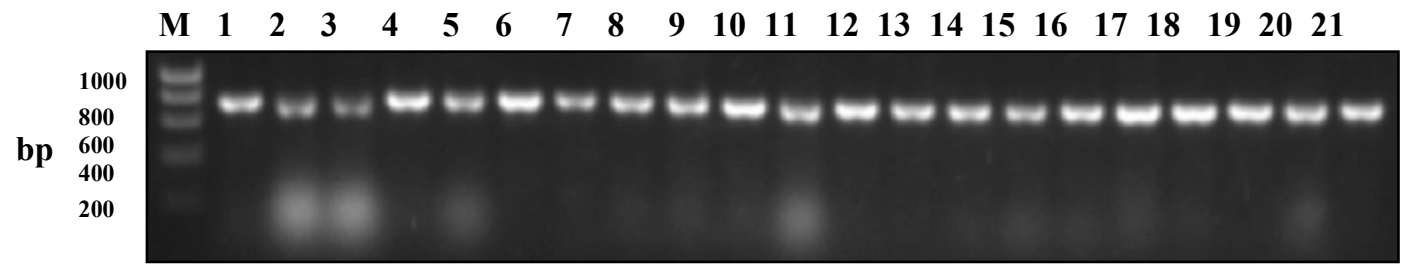

21 randomly selected pale colonies

C) $\mathbf{M} * * * * * * * * * * * * * * * * * * * * *(-)(+)$

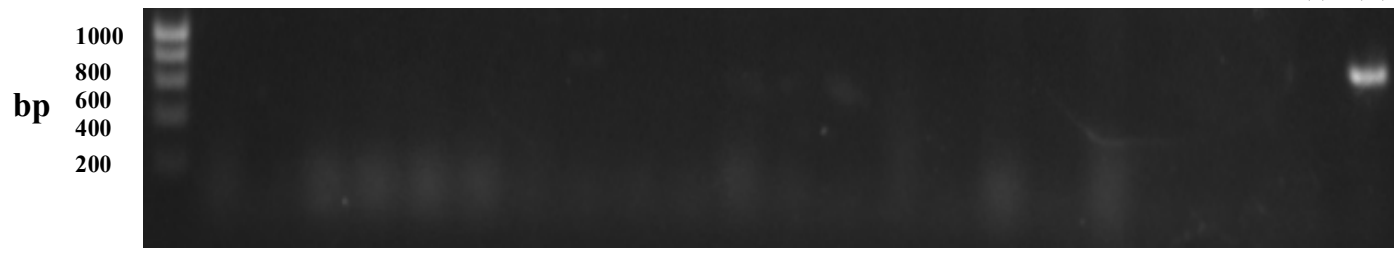

Figure 5.7 - Detection of W3110 colonies from a mixed population of $10 \% \mathrm{~W} 3110$ and $90 \%$ 7TL colonies using 4-nitroimidazole. A) A liquid culture comprising of 10\% W3110 cells and 90\% 7TL cells was plated on LB agar to form colonies. Top agarose containing $20 \mathrm{mM}$ 4-nitroimdazole was poured over these colonies to detect the nitroreductase expression of W3110 cells. A) Twenty-one dark purple colonies (numbered) were selected at random and screened by PCR using $n f_{S} A_{-} E c$ primers. B) The PCR products generated off each of these colonies were run on a corresponding numbered lane on an agarose gel. C) Twenty-one colonies that did not appear to have changed colour were also screened using NfsA_Ec primers and the PCR products were run on another agarose gel. $(-)=$ Template free negative control for both B) and C). $(+)=$ W3110 positive control for both B) and C). 
From these data, it was concluded that 4-nitroimidazole was capable of detecting colonies with low levels of nitroreductase expression against a higher background of colonies lacking any nitroreductase expression, therefore making it a promising tool for the high throughput screening of metagenomic libraries to detect eDNA nitroreductase enzymes. Unfortunately, however, time constraints precluded screening either metagenomic library using this method.

\subsection{Chapter 5 Discussion}

This chapter explored different functional metagenomic screening techniques to discover novel nitroreductase enzymes. We first tested if the activation of FSL41 could be used to detect nitroreductases within eDNA inserts. We envisaged incubating E. coli 7TL containing a metagenomic library in the presence of the profluorophore FSL41, then using FACS to collect the most fluorescent cells; i.e. those that had activated the most profluorophore and therefore potentially contained a nitroreductase.

It was found that activation of the profluorophore FSL41 by the endogenous levels of nitroreductase expression of W3110 cells was sufficient to distinguish them nilnitroreductase strain E. coli 7TL (Figure 5.1). However, this did not necessarily mean an eDNA nitroreductase would also be detectable. The physiological expression levels of NfsA_Ec and NfsB_Ec (the nitroreductase enzymes with the most FSL41 activity) in W3110 cells might still be substantially higher than that of an eDNA nitroreductase. Furthermore, FSL41 was likely being activated by both of these enzymes, rather than just the one which would be expected in an eDNA insert. A better control would perhaps have been a W3110 strain where all nitroreductases other than either NfsA_Ec or $\mathrm{NfsB}$ _Ec had been deleted from the chromosome. Overall though, the experiment demonstrated that nitroreductase overexpression was not required for detectable levels of FSL41 activation, providing support that it could be used to detect the expression of eDNA nitroreductases.

The E. coli 7TL strain used was widely throughout this chapter for the expression of metagenomic libraries. In addition to lacking seven nitroreductase genes, eliminating all background levels of FSL41 activation compared to W3110 cells (Figure 5.1), E. coli 7TL had been modified by lysogenization to express T7 RNA polymerase. As both of the 
metagenomic libraries available had been cloned into plasmids containing $\mathrm{T} 7$ promoter sequences (Owen et al., 2012; Parachin and Gorwa-Grauslund, 2011), the presence of T7 RNA polymerase could increase the chances of expressing eDNA nitroreductases at detectable levels. A proof of concept experiment demonstrated the increased expression of pETDUET_NfsB_Ec in the E. coli 7TL cells relative to the unlysogenized E. coli 7NT control (Figure 5.2). The increased expression of pETDUET_NfsB_Ec was an idealized scenario, however. It was unlikely a nitroreductase within an eDNA insert (typical size range $4-7 \mathrm{~kb}$ ) would be directly adjacent to the $\mathrm{T} 7$ promoter sequence as was the case with pETDUET_NfsB_Ec. Furthermore, expression could be prevented if a transcriptional terminator sequence separated an eDNA nitroreductase from the T7 promoter (Gabor et al., 2004). From this experiment we concluded that T7 RNA polymerase could at least increase the expression of eDNA nitroreductase genes adjacent to a $\mathrm{T} 7$ promoter sequence, potentially enabling their detection.

We next tested if FSL41 could be used to recover E. coli 7TL cells expressing pETDUET_NfsB_Ec via FACS. However, from the results of the control experiments (Figure 5.3) there was no evidence of enrichment of pETDUET_NfsB_Ec. Further optimization would therefore be required for this approach to be feasible as a metagenomic screening technique. A reason for the lack of E. coli 7TL pETDUET NfsB_Ec enrichment could be cell death. While $50 \mu \mathrm{M}$ FSL41 was not a toxic dose to $E$. coli 7TL pETDUET NfsB_Ec in liquid culture, it was possible that, factoring in the additional stress of FACS, these cells were being killed. In which case lower concentrations of FSL41 could be tested in attempt to improve pETDUET NfsB_Ec recovery. Alternatively, FSL41 may not be as cell entrapped as previously observed (Horvat, 2012), resulting in its gradual dissipation from the cell it was activated in and thus preventing detection by FACS. A different cell entrapped profluorophore, such as FSL76, could therefore be tested in the future. Given the time constraints of this work, however, we decided to explore the use of niclosamide as an alternative screening strategy.

As demonstrated in Chapter 3 and Chapter 4, E. coli $\Delta$ tolC clones overexpressing a nitroreductase enzyme were selectively viable in the presence of niclosamide. On the other hand, cells lacking endogenous nitroreductase expression were highly sensitive to niclosamide. We reasoned that E. coli 7TL cells expressing an eDNA nitroreductase 
could therefore be selectively viable when plated on agar containing niclosamide. However, when this technique was tested in the past (Janine Copp, Rory Little, unpublished), the detection of eDNA nitroreductase enzymes was complicated by a high recovery of clones expressing eDNA cellular efflux pumps. We chose to use FSL41 as a counter screen to differentiate niclosamide-resistant eDNA clones expressing nitroreductase enzymes from those expressing cellular efflux pumps. Unfortunately, no eDNA nitroreductase enzymes were detected using FSL41 as a counter-screen for niclosamide-resistant colonies. It is possible that an eDNA nitroreductase capable of detoxifying niclosamide and activating FSL41 is a rare occurrence, simply requiring additional library screening. A range of different profluorophores could also be used for the counter-screening, increasing the chances an eDNA nitroreductase could activate one and be detected.

Alternatively, methods to actively reduce the recovery of eDNA efflux pumps could be tested in the future. For example, LB agar could be amended with broad-spectrum efflux pump inhibitors such as PA $\beta \mathrm{N}$ (Lomovskaya et al., 2001) or pimozide (Bohnert et al., 2013), counteracting efflux pump niclosamide resistance. In addition to reducing the recovery of clones expressing eDNA efflux pumps, other methods could be tested to specifically increase the expression of eDNA nitroreductase enzymes. For example, the expression of NfsA_Ec, implemented in bacterial oxidative stress defense, can be induced by the ROS generator paraquat (Liochev et al., 1999). Oxidative stress could therefore be explored as a method to increase the expression of eDNA nitroreductase enzymes, at least those with a similar biological role to NfsA_Ec.

A limiting factor of both fluorescence and niclosamide-based metagenomic screens was their low throughput nature. Colonies needed to be individually picked in order to run the secondary FSL41 validation assays. We therefore directed our focus to developing highthroughput detection methods of nitroreductase enzymes. The production of $\beta$ galactosidase in response to DNA-damage in the E. coli SOS-R2 strain was briefly considered as a high-throughput metagenomic screening strategy. If successful, thousands of colonies could be screened simultaneously on LB agar plates. The results demonstrated, however, that the basal level of $\beta$-galactosidase expression in SOS-R2 cells was too high for the specific detection of cells expressing a nitroreductase enzyme. Unless basal expression from the SOS promoter could be more tightly controlled, such 
that $\beta$-galactosidase was only expressed in response to a genotoxic prodrug, this screening strategy was unlikely to be successful. As directed evolution had previously reduced the leakiness of genetic control elements (Gatti-Lafranconi et al., 2013), it could also potentially be used reduce leaky expression from the SOS promoter in the future. Regardless, although the SOS-R2/ $\beta$-galactosidase system was ultimately unsuccessful, the detection of nitroreductase expression at the level of a single colony was an attractive screening prospect. This goal was achieved upon the serendipitous discovery that the reduction of 4-nitroimidazole produces a dark purple colour.

In the final section of this chapter, the capabilities of 4-nitroimidazole to detect nitroreductase expression at a single colony level, and therefore serve as a metagenomic screening agent, were tested. The dark purple colour change of 4-nitroimidazole in the presence of E. coli clones overexpressing a nitroreductase occurred rapidly, only requiring around 30 minutes of room temperature incubation; whereas no colour change was observed in E. coli cells lacking nitroreductase expression (Figure 5.5). The lower levels of nitroreductase expression from the endogenous nitroreductases present in W3110 cells were also detectable (Figure 5.6) indicating that nitroreductase overexpression was not required for the colour change.

Colony level detection of nitroreductase expression using a similar colour change had been observed previously, albeit through a different mechanism (Rafil et al., 1991). In that study, the compounds 1-nitropyrene or 6-nitrochrysene were supplemented into LB agar before an intestinal sample of bacteria was added to the plates. Colonies were then exposed to distilled water containing $\mathrm{N}$-(1-Naphthyl)ethylenediamine dihydrochloride (NEDD). Under acidic conditions, the reduced forms 1-nitropyrene or 6-nitrochrysene generated by nitroreductase activity reacted with NEDD to form a red/purple azo dye, the formation of which was detectable as change in colony colour (Rafil et al., 1991). The 4nitroimidazole detection method developed in this study has the benefit of only requiring a single step - exposure of colonies to the compound in top agarose - for the colour change to occur. However, both methods could be concurrently tested to determine if one were more sensitive than the other and therefore better suited for metagenomic screening. It would also be interesting to determine the underlying mechanism responsible for the colour change of 4-nitroimidazole, especially since no colour change was observed in the presence of purified NfsA_Ec alone (Michelle Rich, unpublished), suggesting further 
reactive steps. Furthermore, the same study demonstrated that when bacteria were plated on LB agar containing 1-nitropyrene (a yellow compound), zones of 1-nitropyrene clearance were observed if the bacteria were expressing a nitroreductase enzyme (Rafil et al., 1991). Plating a metagenomic library LB agar containing 1-nitropyrene to observe zones of clearance could therefore also be tested as a metagenomic screening strategy in future.

Although not possible due to the time constraints of this research, the ultimate validation of 4-nitroimidazole as a metagenomic screening tool would require it be used to discover an eDNA nitroreductase enzyme. Previous metagenomic screens utilizing changes in colony colour as a positive marker for an enzyme of interest were, encouragingly, successful (Owen et al., 2012; Wang et al., 2010). Much will depend on whether the nitroreductases present within a metagenomic library are capable of activating 4nitroimidazole. As members of the NfsA family are generally the most active with 4nitroimidazole (Condon, 2013), using it to screen metagenomic libraries could introduce a selection bias towards NfsA enzymes. However, as NfsA family enzymes are among the most active with nil-bystander prodrugs such as metronidazole, specifically finding new members of this family could be particularly beneficial for future zebrafish cell ablation studies. In additional work by Dr. Elsie Williams (unpublished), colonies overexpressing NfsA_Ec were found to turn green in the presence of the metronidazole analogue 2methyl-5-nitroimidazole. As some nitroreductase enzymes within eDNA inserts may not activate 4-nitroimidazole, but could potentially activate 2-methyl-5-nitroimidazole, simultaneously exposing metagenomic libraries to both prodrugs could increase the chances of discovery. There are examples of such nitroreductases in our own 58 core nitroreductase library, such as YwrO from Listeria innocua (Condon, 2013). Collectively, the results in this chapter were supportive that 4-nitroimidazole could be successfully used to find nitroreductases in metagenomic libraries in the near future. 


\section{Chapter 6 Research Motivation, Key Findings, and Future Directions}

\subsection{Research motivation}

The primary motivation behind this research was to engineer and discover new nitroreductase enzymes to improve targeted cell ablation studies in zebrafish. While research has been conducted to engineer (Grove et al., 2003; Guise et al., 2007; Swe et al., 2012) and discover (Prosser et al., 2013, 2010) superior nitroreductases for GDEPT, the same is not true for targeted cell ablation studies. Indeed, the one example where an engineered nitroreductase was expressed in zebrafish for targeted cell ablation, the enzyme had originally been evolved for a GDEPT application (Mathias et al., 2014). As targeted cell ablation in zebrafish is an important tool for studying regenerative processes (Slanchev et al., 2005; White and Mumm, 2013) and modeling human diseases (Pisharath et al., 2007; Zhou and Hildebrandt, 2012), we were motivated to develop new nitroreductase enzymes tailored to such studies.

We directed our focus to developing a cell ablation system with multiplex capabilities, providing temporal control over the independent ablation of two distinct tissues within the same organism. A multiplex ablation system could be useful for studying the interaction between different tissues during physiological processes such as embryonic development. Furthermore, as nitroreductase enzymes can activate nitroaromatic profluorophore molecules, the system also potentially had intrinsic imaging capabilities. For multiplex ablation to be possible, two nitroreductase enzymes, each possessing nonoverlapping prodrug selectivities were required. Previous findings by the Ackerley lab had identified pairs of nitroreductase enzymes demonstrating a limited degree of nonoverlapping selectivity for the prodrugs metronidazole and tinidazole. The goal of this work was to expand upon these previous findings and use directed evolution to further enhance the prodrug selectivity of these enzymes, thus making them better suited for a multiplex cell ablation system. As an alternative to engineering novel nitroreductases for cell ablation studies, techniques to discover novel nitroreductase enzymes within metagenomic libraries were also explored. 


\subsection{Key Findings}

The first directed evolution experiment performed (Chapter 3) was to increase the tinidazole selectivity of NfsA_Ec. NfsA_Ec initially only possessed a very slight bias towards tinidazole activiation compared to metronidazole activation. It would therefore be not be suitable for inclusion in a multiplex ablation system opposite our lead metronidazole selective candidate, $\mathrm{NfsB}$-Vv. We chose to use directed evolution to improve the tinidazole selectivity of NfsA_Ec.

The directed evolution screen developed consisted of challenging the NfsA_Ec 7SM library using niclosamide and metronidazole in combination. Niclosamide was found to be an effective positive selection agent for catalytically active NfsA_Ec 7SM mutants. In contrast, metronidazole could be used to select against NfsA mutants possessing wild type levels of metronidazole activity. The enzyme AzoR_Ec was a valuable control for optimizing the concentrations of niclosamide and metronidazole ultimately used for library screening.

Plating SOS-R4 E. coli cells containing the NfsA_Ec 7SM library on LB agar containing metronidazole and niclosamide enabled the successful evolution of NfsA_Ec enzymes that had improved tinidazole selectivity. Of the nine NfsA_Ec 7SM mutants analyzed, the fold increases in metronidazole $\mathrm{IC}_{50}$ relative to wild type NfsA_Ec ranged from 12-71 $($ mean $=32)$, while the fold increases in tinidazole $\mathrm{IC}_{50}$ ranged from 3-20 (mean $=6$ ). Measuring protein expression of these mutants relative to NfsA_Ec indicated that catalytic changes, rather than changes in expression level, were likely responsible for the altered prodrug selectivity. The most promising mutant recovered, NfsA_Ec 7SM 5_B9, had a 31 fold higher metronidazole $\mathrm{IC}_{50}$ than NfsA_Ec, while only a 3 fold higher tinidazole $\mathrm{IC}_{50}$, overall making it ca. 12 fold more tinidazole selective.

Testing each codon change present in the NfsA_Ec 7SM 5_B9 mutant in isolation indicated that no single amino acid change was responsible for the increase in tinidazole selectivity. The change in selectivity was therefore likely representative of synergistic effects between the multiple amino acid changes. 
Unfortunately, all of the top tinidazole selective NfsA_Ec 7SM mutants had also lost activity with the profluorophores FSL76 and FSL150, meaning multiplex imaging in combination with the metronidazole selective NfsB_Vv was unlikely to be possible.

A second directed evolution experiment was performed (Chapter 4) to increase the tinidazole selectivity of NfsA_Pp. NfsA_Pp was selected because it was one of the most intrinsically tinidazole selective enzymes present in the 58 core nitroreductase library (Condon, 2013). From the $\mathrm{IC}_{50}$ data measured in this thesis, NfsA_Pp was two fold more tinidazole selective than NfsA_Ec. A library of NfsA_Pp mutants was created using error prone PCR and screened by plating on LB agar containing metronidazole and niclosamide. While the screen successfully selected against metronidazole activity, tinidazole activity was also greatly decreased compared to wild type NfsA_Pp. Overall none of NfsA_Pp mutants identified were notable more tinidazole selective than wild type NfsA_Pp.

The goal of the research described in the final chapter of this thesis (Chapter 5) was to discover new nitroreductase enzymes from within metagenomic libraries. Several different nitroreductase metagenomic screening techniques were developed and tested. Unfortunately, the approaches using the profluorophore FSL41, niclosamide, or $\beta$ galactosidase expression from the E. coli SOS-R2 strain were all ultimately unsuccessful. The most promising discovery made in this chapter was that the compound 4nitroimidazole could detect nitroreductase expression at the level of a single colony. While timing constraints prevented a large-scale library screen, preliminary results were supportive that 4-nitroimidazole could be used for discovering metagenomic nitroreductase genes in the near future. 


\subsection{Future directions}

\subsubsection{Alternative Prodrug Multiplex Ablation Systems}

As discussed in Chapter 3, metronidazole and tinidazole were chosen to develop a multiplex ablation system around because they are both affordable and well-characterized nitroaromatic prodrugs. Metronidazole was also the standard prodrug used in cell ablation studies, meaning it would be familiar to members of the field. Despite these attractive practical considerations, it could be that alternative prodrug pairings would be better suited for a multiplex ablation system. Given that metronidazole and tinidazole were both 5-nitroimidazole prodrugs, it is conceivable that they interacted with the active site of a nitroreductase in a similar fashion. The common 5-nitroimidazole scaffold of the two prodrugs could therefore potentially restrict the upper limit of selectivity a nitroreductase could possess for either of them. In contrast, a nitroreductase might activate two nil-bystander prodrugs that have completely different structures with a much higher level of discrimination. Previous results from the Ackerley lab support this scenario. For example, the metronidazole selective NfsB_Vv was even less active with the 2-nitroimidazole fluorinated misonidazole (F-Miso) than it was with tinidazole (Condon, 2013). Future studies to evolve nitroreductase enzymes for multiplex ablation may therefore be more successful if the prodrugs used differed greatly from one another. However, this would require comprehensive validation of both prodrugs for effective targeted cellular ablation, and this has not yet been obtained for fluorinated misonidazole.

Prodrugs could even be foregone in future systems, replaced with nitroreductase-caged circularized morpholino oligonucleotides. Morpholino oligonucleotides are synthetic single-stranded nuclease-resistant nucleic acid analogues that can prevent translation of a specific zebrafish gene by hybridizing to its mRNA (Bill et al., 2009). In a recent study, morpholinos were inactivated by circularization using a nitrobenzyl linker (Yamazoe et al., 2014). Reduction of the linker by the nitroreductase enzyme NfsB_Ec resulted in decircularization of the morpholino, and subsequent gene knockdown in zebrafish. The creation of alternative nitroaromatic linker groups (e.g., based on the 2-nitroimidazole linker used to quench the fluorescence of FSL178; see structure, Figure 3.21), could potentially enable the creation of a multiplex gene knockdown system, where one 
nitroreductase expressed from one tissue would specifically decircularize one morpholino, and one expressed from another tissue would specifically decircularize another. Such a system would provide temporal control over the knockdown of two specific genes in two distinct tissues of the same organism. If achievable, this system could be used to study genetic interactions (such as synthetic lethality) in zebrafish model organisms, an area of interest to the field (Hajeri and Amatruda, 2012).

\subsubsection{Future Directed Evolution Experiments to Enhance Substrate Selectivity}

The results from Chapter $\mathbf{3}$ demonstrated that using metronidazole as a negative selection agent against the NfsA_Ec 7SM mutant library was far more detrimental to metronidazole activity than to tinidazole activity. This raises the question as to whether negative selection could be used to select against multiple prodrug activities simultaneously. For example, the NfsA_Ec 7SM library could be plated on agar supplemented with metronidazole and another prodrug, such as fluorinated misonidazole, in addition to niclosamide. Ultimately, such an approach might lead to development of three different nitroreductase enzymes each having unique and non-overlapping prodrug selectivities, that could be employed in a three-way multiplex ablation system. While speculating about the degree to which substrate selectivity could be narrowed (what about selecting against three prodrug activities? How about four or five?) is exciting from enzyme engineer's perspective, it is important to keep an application in mind. Ultimately, the enzymes evolved need to be of practical use to the field of targeted cell ablation. Only time will tell if larger and more complex multiplex ablation systems would be useful to the field.

\subsubsection{Exploring the Mechanistic Basis Behind Substrate Selectivity}

The relative metronidazole and tinidazole selectivities of the nitroreductase enzymes considered in this thesis were all based on empirical data. The underlying mechanistic reasons why enzymes such as $\mathrm{NfsB}$ _Vv demonstrate greater activity with metronidazole compared to tinidazole, despite them being structurally similar 5-nitroimidazole prodrugs, was unknown. These questions could be answered by co-crystallizing a nitroreductase with either metronidazole or tinidazole. Analyzing such crystals could provide a structural and thermodynamic basis to explain why one prodrug is preferentially activated 
by one nitroreductase enzyme and not another. In ongoing work, Ackerley lab $\mathrm{PhD}$ student Abby Sharrock plans to answer some of these questions by further engineering, and ultimately crystallization, of the tinidazole selective NfsA_Ec 7SM mutants generated in this thesis. A potential outcome from this research could be the identification of key amino acids that dictate nitroreductase 5-nitroimidazole selectivity. These amino acids could then be specifically targeted for mutagenesis in future evolution experiments to potentially yield even greater gains in substrate selectivity.

\subsubsection{Multiplex Imaging using Fluorescent Proteins}

The concept of a multiplex imaging and ablation system was considered in Section 3.2.10 of this thesis. Bacterial fluorescence assays revealed that the top tinidazole selective NfsA_Ec 7SM mutants had all lost activity with the (generally NfsA selective) profluorophores FSL76 and FSL150. While multiplex imaging may not be achievable using these profluorophores, it could possibly be achieved using fluorescent proteins. Nitroreductase enzymes have been successfully fused to multiple fluorescent proteins, such as CFP (Curado et al., 2007) and GFP (Hsu et al., 2010). Methodologies also exist for simultaneously imaging two district zebrafish tissues expressing different fluorescent protein markers (Ariga et al., 2010). For example, NfsB_Vv could be fused to GFP while a tinidazole selective NfsA_Ec mutant could be fused to CFP, enabling the specific imaging of each. Alternatively, if the creation of a fusion protein was detrimental to nitroreductase activity, each nitroreductase could be co-expressed along with a distinct fluorescent protein from a bicistronic promoter.

\subsubsection{Metagenomic Screening using Chloramphenicol}

In regards to the metagenomic discovery aim of this project, nitroreductase positive selection agents other than niclosamide could be tested in the future. Ideally, these positive selection agents would not be dependent on the absence of TolC for toxicity, preventing the unwanted recovery of eDNA efflux pumps. Preliminary data collected by Dr. Elsie Williams (unpublished) has indicated that the antibiotic chloramphenicol can also be a positive selection agent of cell expressing nitroreductase enzymes. The reduction of the nitro group of chloramphenicol can decrease its antimicrobial activity (Corbett and Chipko, 1978), providing a possible explanation for its positive selection 
activity. Encouragingly, the positive selection effect of chloramphenicol was still observed when a functional TolC transporter was present in the host E. coli cell. Chloramphenicol could therefore be tested in the future as an alternative metagenomic screening tool to niclosamide.

\subsubsection{Metagenomic Screening using Degenerate Nitroreductase Primers}

An alternative to the functional-based metagenomic screens developed in this thesis could be a homology-based approach using degenerate PCR primers. Degenerate PCR primers are broadly specific for a given gene family by targeting conserved sequence domains. Their broadly specific nature enables them to be used to screen metagenomic libraries for new members of the same gene family. An advantage of a degenerate primer approach over functional screens is that eDNA gene expression is not required for detection (Piel, 2011). Degenerate primers have previously been successfully used to identify new members of the dehalosgenase (Chae et al., 2008), chitinase (Cottrell et al., 2000), protease (Niehaus et al., 2011), and xylose isomerase (Parachin and Gorwa-Grauslund, 2011) gene families. In previous work, degenerate $n f s B$ primers were designed that were capable of detecting $\mathrm{nfsB}$ genes from eight different bacterial species (Rory Little and David Ackerley, unpublished). These primers could therefore be used to screen metagenomic libraries for novel $n f_{s} B$ genes in the future.

\subsubsection{Using 4-Nitroimidazole as a Directed Evolution Tool}

While this thesis only considered the prodrug 4-nitroimidazole in a metagenomic screening context, it could also be a useful tool for directed evolution studies. The 4nitroimidazole-induced colour change of an E. coli colony expressing a nitroreductase could be used to rapidly detect nitroreductase mutants that had either lost or gained activity with 4-nitroimidazole. Such a screening technique could be useful for evolving alternative pairs of multiplex ablation nitroreductase enzymes. For example, as successfully demonstrated in this thesis, metronidazole could be used as a negative selection agent to select against mutants possessing metronidazole activity. These same mutants could then be rapidly counter-screened to identify those that retained activity with 4-nitroimidazole. The reverse could then be done, supplementing agar with inhibitory levels of 4-nitroimidazole to eliminate mutants capable of activating it. The 
metronidazole activity of these 4-nitroimidazole inactive mutants could then be assessed by metronidazole SOS GFP assays. The end result would be two enzymes with nonoverlapping 4-nitroimidazole and metronidazole activities.

The colour change of 4-nitroimidazole could also enable it to be used as a neutral drift evolution tool. Neutral drift evolution is useful when the desired activity of the enzyme is not amenable to direct screening. In these cases, mutant enzymes can be selected on the basis that they have retained functionality with a different substrate, i.e. all mutations that abrogated enzyme function altogether have been eliminated from the pool. Successive rounds of evolution selecting enzymes for one functionality can inadvertently result in improvements in a different desired, but difficult to detect, activity (Bershtein and Tawfik, 2008). Thus, 4-nitroimidazole may have value in selecting for enzymes that are active with entirely different prodrug substrates, akin to the use of niclosamide in this thesis. Moreover, 4-nitroimidazole may have more direct value as an analogue of compounds that possess a core 4-nitroimidazole scaffold. Another project in the Ackerley lab has focused on developing methods to detect the expression of a nitroreductase protein, delivered by an oncolytic virus or bacteria, in the cancerous tissue of a GDEPT patient. The ability to verify that nitroreductase expression is restricted to cancerous tissue prior to administration of a prodrug is an important regulatory hurdle for GDEPT (Williams, 2013). A promising method for achieving this is positron emission tomography (PET), using radioactively labelled nitroaromatic probes that, upon nitroreduction, become cell entrapped. Dr Ackerley's collaborators at the ACSRC have synthesized a range of PET-capable probes based on nitroimidazole scaffolds. In ongoing work, directed evolution has been performed on nitroreductase enzymes to enhance their activity with these PET probes. Unfortunately, while these PET probes can inhibit bacterial growth, many do not induce an SOS response, making screening for improved mutants difficult. Growth inhibition assays are often not practical for large-scale screening due to the high costs and low availability of these bespoke preclinical compounds. However, as several of the most promising GDEPT PET probes contain core 4-nitroimidazole scaffolds, the colour change of 4-nitroimidazole could be used to select for mutants possessing at least some level of generic 4-nitroimidazole activity. A mutant nitroreductase library could therefore be screened to identify the mutants best able to reduce 4-nitroimidazole. These mutants could then be subjected to further rounds of 
mutagenesis and selection; likely to ultimately result in the enrichment of mutants better at activating the 4-nitroimidazole PET probes.

\subsection{Concluding Remarks}

This work built off previous studies demonstrating that different nitroreductase enzymes can selectively activate some prodrugs over others (Condon, 2013; Horvat, 2012). A key goal of this study was to determine if directed evolution could be performed to further enhance this selectivity. The approach that was developed in this thesis successfully enabled the evolution of an NfsA_Ec mutant possessing decreased levels of metronidazole activity and therefore enhanced tinidazole selectivity, the first example of its kind. To fully validate these evolved enzymes as useful multiplex ablation tools, however, they will need to be expressed in the tissues of the intended model organisms, zebrafish in particular. The bacterial data collected in this thesis is nonetheless supportive that engineered nitroreductases could be used to expand the capabilities of future cell ablation studies. Towards the other goal of this thesis - the discovery of nitroreductase enzymes in metagenomic libraries - the compound 4-nitroimidazole also offers promise that novel nitroreductase enzymes from metagenomic libraries will be discoverable in the future.

Overall, this thesis expanded our knowledge on the nitroreductase activity changes achievable using directed evolution and methods that could be used to detect novel nitroreductase enzymes in metagenomic libraries. 


\section{References}

Ackerley, D.F., Gonzalez, C.F., Park, C.H., Blake, R., Keyhan, M., Matin, A., 2004. Chromate-reducing properties of soluble flavoproteins from Pseudomonas putida and Escherichia coli. Appl. Environ. Microbiol. 70, 873-882.

Aharoni, A., Gaidukov, L., Khersonsky, O., Gould, S.M., Roodveldt, C., Tawfik, D.S., 2005. The "Evolvability" of Promiscuous Protein Functions. Nat. Genet. 37, 7376.

Ahmad, I., Ahmad, F., Pichtel, J., 2011. Microbes and Microbial Technology: Agricultural and Environmental Applications. Springer Science \& Business Media.

Ariga, J., Walker, S.L., Mumm, J.S., 2010. Multicolor Time-lapse Imaging of Transgenic Zebrafish: Visualizing Retinal Stem Cells Activated by Targeted Neuronal Cell Ablation. J. Vis. Exp. JoVE.

Armstrong, N.R., Wilson, J.D., 2010. Tinidazole in the Treatment of Bacterial Vaginosis. Int. J. Womens Health 1, 59-65.

Baharoglu, Z., Mazel, D., 2014. SOS, the Formidable Strategy of Bacteria Against Aggressions. FEMS Microbiol. Rev. 38, 1126-1145.

Bai, S.Y., Denny, W.A., Li, D., Patterson, A.V., Shen, Y., Singleton, D.C., Smaill, J.B., Syddall, S.P., Wilson, W.R., 2007. The Nitroreductase Prodrug SN 28343 Enhances the Potency of Systemically Administered Armed Oncolytic Adenovirus ONYX-[411.sup.NTR]. Cancer Gene Ther. 14, 953+.

Barak, Y., Thorne, S.H., Ackerley, D.F., Lynch, S.V., Contag, C.H., Matin, A., 2006a. New Enzyme for Reductive Cancer Chemotherapy, YieF, and its Improvement by Directed Evolution. Mol. Cancer Ther. 5, 97-103.

Barak, Y., Ackerley, D.F., Dodge, C.J., Banwari, L., Alex, C., Francis, A.J., Matin, A., 2006b. Analysis of Novel Soluble Chromate and Uranyl Reductases and Generation of an Improved Enzyme by Directed Evolution. Appl. Environ. Microbiol. 72, 7074-7082.

Bargmann, C.I., Avery, L., 1995. Laser Killing of Cells in Caenorhabditis elegans. Methods Cell Biol. 48, 225-250.

Berrutti, M., Pellicano, R., Astegiano, M., Smedile, A., Saracco, G., Morgando, A., De Angelis, C., Repici, A., Fagoonee, S., Leone, N., Rizzetto, M., 2008. Helicobacter pylori Eradication: Metronidazole or Tinidazole? Data from Turin, Italy. Minerva Gastroenterol. Dietol. 54, 355-358.

Bershtein, S., Tawfik, D.S., 2008. Advances in Laboratory Evolution of Enzymes. Curr. Opin. Chem. Biol., Biocatalysis and Biotransformation/Bioinorganic chemistry $12,151-158$.

Bhaumik, S., Sekar, T.V., Depuy, J., Klimash, J., Paulmurugan, R., 2012. Noninvasive Optical Imaging of Nitroreductase Gene-Directed Enzyme Prodrug Therapy System in Living Animals. Gene Ther. 19, 295-302.

Bill, B.R., Petzold, A.M., Clark, K.J., Schimmenti, L.A., Ekker, S.C., 2009. A Primer for Morpholino Use in Zebrafish. Zebrafish 6, 69-77.

Blattner, F.R., Plunkett, G., Bloch, C.A., Perna, N.T., Burland, V., Riley, M., ColladoVides, J., Glasner, J.D., Rode, C.K., Mayhew, G.F., Gregor, J., Davis, N.W., Kirkpatrick, H.A., Goeden, M.A., Rose, D.J., Mau, B., Shao, Y., 1997. The Complete Genome Sequence of Escherichia coli K-12. Science 277, 1453-1462. 
Bocola, M., Otte, N., Jaeger, K.-E., Reetz, M.T., Thiel, W., 2004. Learning from Directed Evolution: Theoretical Investigations into Cooperative Mutations in Lipase Enantioselectivity. ChemBioChem 5, 214-223.

Bohnert, J.A., Schuster, S., Kern, W.V., 2013. Pimozide Inhibits the AcrAB-TolC Efflux Pump in Escherichia coli. Open Microbiol. J. 7, 83-86.

Brady, S.F., Chao, C.J., Clardy, J., 2004. Long-Chain N-Acyltyrosine Synthases from Environmental DNA. Appl. Environ. Microbiol. 70, 6865-6870.

Bridgewater, J.A., Knox, R.J., Pitts, J.D., Collins, M.K., Springer, C.J., 1997. The Bystander Effect of the Nitroreductase/CB 1954 Enzyme/Prodrug System Is Due to a Cell-Permeable Metabolite. Hum. Gene Ther. 8, 709-717. doi:10.1089/hum.1997.8.6-709

Bridgewater, J.A., Springer, C.J., Knox, R.J., Minton, N.P., Michael, N.P., Collins, M.K., 1995. Expression of the Bacterial Nitroreductase Enzyme in Mammalian Cells Renders them Selectively Sensitive to Killing by the Prodrug CB1954. Eur. J. Cancer Oxf. Engl. 1990 31A, 2362-2370.

Cattaneo, R., Miest, T., Shashkova, E.V., Barry, M.A., 2008. Reprogrammed Viruses as Cancer Therapeutics: Targeted, Armed and Shielded. Nat. Rev. Microbiol. 6, 529-540.

Chae, J.-C., Song, B., Zylstra, G.J., 2008. Identification of Genes Coding for Hydrolytic Dehalogenation in the Metagenome Derived from a Denitrifying 4Chlorobenzoate Degrading Consortium. FEMS Microbiol. Lett. 281, 203-209.

Chen, C.-F., Chu, C.-Y., Chen, T.-H., Lee, S.-J., Shen, C.-N., Hsiao, C.-D., 2011. Establishment of a Transgenic Zebrafish Line for Superficial Skin Ablation and Functional Validation of Apoptosis Modulators In Vivo. PLoS ONE 6, e20654.

Chica, R.A., Doucet, N., Pelletier, J.N., 2005. Semi-Rational Approaches to Engineering Enzyme Activity: Combining the Benefits of Directed Evolution and Rational Design. Curr. Opin. Biotechnol., Protein Technologies and Commercial Enzymes $16,378-384$.

Christofferson, A., Wilkie, J., 2009. Mechanism of CB1954 Reduction by Escherichia coli Nitroreductase. Biochem. Soc. Trans. 37, 413.

Chu, X., He, H., Guo, C., Sun, B., 2008. Identification of Two Novel Esterases from a Marine Metagenomic Library Derived from South China Sea. Appl. Microbiol. Biotechnol. 80, 615-625.

Clark, A.J., Iwobi, M., Cui, W., Crompton, M., Harold, G., Hobbs, S., Kamalati, T., Knox, R., Neil, C., Yull, F., Gusterson, B., 1997. Selective Cell Ablation in Transgenic Mice Expressing E. coli nitroreductase. Gene Ther. 4, 101-110.

Cobb, R.E., Sun, N., Zhao, H., 2013. Directed Evolution as a Powerful Synthetic Biology Tool. Methods San Diego Calif 60, 81-90.

Collier, R.J., 2001. Understanding the Mode of Action of Diphtheria toxin: a Perspective on Progress During the $20^{\text {th }}$ Century. Toxicon 39, 1793-1803.

Condon, S., 2013. The Characterisation and Application of Nitroreductase Enzymes.

Copp, J.N., Williams, E.M., Rich, M.H., Patterson, A.V., Smaill, J.B., Ackerley, D.F., 2014. Toward a High-Throughput Screening Platform for Directed Evolution of Enzymes that Activate Genotoxic Prodrugs. Protein Eng. Des. Sel. PEDS 27, 399-403.

Corbett, M.D., Chipko, B.R., 1978. Synthesis and Antibiotic Properties of Chloramphenicol Reduction Products. Antimicrob. Agents Chemother. 13, 193198.

Cortial, S., Chaignon, P., Iorga, B.I., Aymerich, S., Truan, G., Gueguen-Chaignon, V., Meyer, P., Moréra, S., Ouazzani, J., 2010. NADH Oxidase Activity of Bacillus 
subtilis Nitroreductase NfrA1: Insight into its Biological Role. FEBS Lett. 584, 3916-3922.

Cottrell, M.T., Wood, D.N., Yu, L., Kirchman, D.L., 2000. Selected Chitinase Genes in Cultured and Uncultured Marine Bacteria in the $\alpha$ - and $\gamma$-Subclasses of the Proteobacteria. Appl. Environ. Microbiol. 66, 1195-1201.

Cui, L., Zhong, Y., Zhu, W., Xu, Y., Du, Q., Wang, X., Qian, X., Xiao, Y., 2011. A New Prodrug-Derived Ratiometric Fluorescent Probe for Hypoxia: High Selectivity of Nitroreductase and Imaging in Tumor Cell. Org. Lett. 13, 928-931.

Cui, W., Allen, N.D., Skynner, M., Gusterson, B., Clark, A.J., 2001. Inducible Ablation of Astrocytes Shows that these Cells are Required for Neuronal Survival in the Adult Brain. Glia 34, 272-282.

Cui, W., Gusterson, B., Clark, A.J., 1999. Nitroreductase-Mediated Cell Ablation is Very Rapid and Mediated by a p53-Independent Apoptotic Pathway. Gene Ther. 6, 764-770.

Curado, S., Anderson, R.M., Jungblut, B., Mumm, J., Schroeter, E., Stainier, D.Y.R., 2007. Conditional Targeted Cell Ablation in Zebrafish: A New Tool for Regeneration Studies. Dev. Dyn. 236, 1025-1035.

Curado, S., Stainier, D.Y.R., Anderson, R.M., 2008. Nitroreductase-Mediated Cell/Tissue Ablation in Zebrafish: a Spatially and Temporally Controlled Ablation Method with Applications in Developmental and Regeneration Studies. Nat. Protoc. 3, 948-954.

Dachs, G.U., Hunt, M.A., Syddall, S., Singleton, D.C., Patterson, A.V., 2009. Bystander or No Bystander for Gene Directed Enzyme Prodrug Therapy. Molecules 14, 4517-4545.

Dale, L.D., Widdick, D.A., Edwards, D.I., Biol, G.I., 1989. Comparative DNA Damage and Repair Induced by Misonidazole, CB1954 and RSU 1069. Int. J. Radiat. Oncol. Biol. Phys. 16, 995-999.

Dang, L.H., Bettegowda, C., Huso, D.L., Kinzler, K.W., Vogelstein, B., 2001. Combination Bacteriolytic Therapy for the Treatment of Experimental Tumors. Proc. Natl. Acad. Sci. 98, 15155-15160.

De Oliveira, I.M., Bonatto, D., Henriques, J.A.P., 2010a. Nitroreductases: Enzymes with Environmental, Biotechnological and Clinical Importance. Curr. Res. Technol. Educ. Top. Appl. Microbiol. Microb. Biotechnol. 3, 6.

De Oliveira, I.M., Zanotto-Filho, A., Moreira, J.C.F., Bonatto, D., Henriques, J.A.P., 2010b. The role of two Putative Nitroreductases, Frm $2 p$ and Hbn1p, in the Oxidative Stress Response in Saccharomyces cerevisiae. Yeast Chichester Engl. 27, 89-102.

Edwards, D.I., 1980. Mechanisms of Selective Toxicity of Metronidazole and Other Nitroimidazole Drugs. Br. J. Vener. Dis. 56, 285-290.

Fasan, R., Chen, M.M., Crook, N.C., Arnold, F.H., 2007. Engineered AlkaneHydroxylating Cytochrome P450BM3 Exhibiting Nativelike Catalytic Properties. Angew. Chem. Int. Ed. 46, 8414-8418.

Felmer, R., Cui, W., Clark, A.J., 2002. Inducible Ablation of Adipocytes in Adult Transgenic Mice Expressing the E. coli nitroreductase gene. J. Endocrinol. 175, 487-498.

Felmer, R.N., Clark, J.A., 2004. The Gene Suicide System NTR/CB1954 Causes Ablation of Differentiated 3T3L1 Adipocytes by Apoptosis. Biol. Res. 37, 449460.

Forbes, N.S., 2010. Engineering the Perfect (Bacterial) Cancer Therapy. Nat. Rev. Cancer $10,785-794$. 
Frayha, G.J., Smyth, J.D., Gobert, J.G., Savel, J., 1997. The Mechanisms of Action of Antiprotozoal and Anthelmintic Drugs in Man. Gen. Pharmacol. Vasc. Syst. 28, 273-299.

Gabor, E.M., Alkema, W.B.L., Janssen, D.B., 2004. Quantifying the Accessibility of the Metagenome by Random Expression Cloning Techniques. Environ. Microbiol. 6, 879-886.

Gatti-Lafranconi, P., Dijkman, W.P., Devenish, S.R., Hollfelder, F., 2013. A Single Mutation in the Core Domain of the lac Repressor reduces Leakiness. Microb. Cell Factories 12, 67.

Golan, D.E., Tashjian, A.H., Armstrong, E.J., 2011. Principles of Pharmacology: The Pathophysiologic Basis of Drug Therapy. Lippincott Williams \& Wilkins.

Gould, S.M., Tawfik, D.S., 2005. Directed Evolution of the Promiscuous Esterase Activity of Carbonic Anhydrase II. Biochemistry (Mosc.) 44, 5444-5452.

Gray, C., Loynes, C.A., Whyte, M.K., Crossman, D.C., Renshaw, S.A., Chico, T.J., 2011. Simultaneous Intravital Imaging of Macrophage and Neutrophil Behaviour During Inflammation Using a Novel Transgenic Zebrafish. Thromb. Haemost. 105, 811.

Green, N.K., Youngs, D.J., Neoptolemos, J.P., Friedlos, F., Knox, R.J., Springer, C.J., Anlezark, G.M., Michael, N.P., Melton, R.G., Ford, M.J., Young, L.S., Kerr, D.J., Searle, P.F., 1997. Sensitization of Colorectal and Pancreatic Cancer Cell Lines to the Prodrug 5-(Aziridin-1-yl)-2,4-Ninitrobenzamide (CB1954) by Retroviral Transduction and Expression of the E. coli Nitroreductase Gene. Cancer Gene Ther. 4, 229-238.

Grove, J.I., Lovering, A.L., Guise, C., Race, P.R., Wrighton, C.J., White, S.A., Hyde, E.I., Searle, P.F., 2003. Generation of Escherichia coli Nitroreductase Mutants Conferring Improved Cell Sensitization to the Prodrug CB1954. Cancer Res. 63, 5532-5537.

Guise, C.P., Grove, J.I., Hyde, E.I., Searle, P.F., 2007. Direct Positive Selection for Improved Nitroreductase Variants using SOS Triggering of Bacteriophage Lambda Lytic Cycle. Gene Ther. 14, 690-698.

Guo, T., Cui, L., Shen, J., Zhu, W., Xu, Y., Qian, X., 2013. A Highly Sensitive LongWavelength Fluorescence Probe for Nitroreductase and Hypoxia: Selective Detection and Quantification. Chem. Commun. 49, 10820-10822.

Hajeri, V.A., Amatruda, J.F., 2012. Studying Synthetic Lethal Interactions in the Zebrafish System: Insight into Disease Genes and Mechanisms. Dis. Model. Mech. 5, 33-37.

Hannink, N., Rosser, S.J., French, C.E., Basran, A., Murray, J.A.H., Nicklin, S., Bruce, N.C., 2001. Phytodetoxification of TNT by Transgenic Plants Expressing a Bacterial Nitroreductase. Nat. Biotechnol. 19, 1168-1172.

Harris, J.A., Cheng, A.G., Cunningham, L.L., MacDonald, G., Raible, D.W., Rubel, E.W., 2003. Neomycin-Induced Hair Cell Death and Rapid Regeneration in the Lateral Line of Zebrafish (Danio rerio). J. Assoc. Res. Otolaryngol. 4, 219-234.

Haynes, C.A., Koder, R.L., Miller, A.-F., Rodgers, D.W., 2002. Structures of Nitroreductase in Three States: Effects of Inhibitor Binding and Reduction. J. Biol. Chem. 277, 11513-11520.

Helsby, N.A., Ferry, D.M., Patterson, A.V., Pullen, S.M., Wilson, W.R., 2004. 2-Amino Metabolites are Key Mediators of CB1954 and SN23862 Bystander Effects in Nitroreductase GDEPT. Br. J. Cancer 90, 1084-1092.

Helsby, N.A., Wheeler, S.J., Pruijn, F.B., Palmer, B.D., Yang, S., Denny, W.A., Wilson, W.R., 2003. Effect of Nitroreduction on the Alkylating Reactivity and Cytotoxicity of the 2,4-Dinitrobenzamide-5-Aziridine CB1954 and the 
Corresponding Nitrogen Mustard SN23862: Distinct Mechanisms of Bioreductive Activation. Chem. Res. Toxicol. 16, 469-478.

Hess, M., Sczyrba, A., Egan, R., Kim, T.-W., Chokhawala, H., Schroth, G., Luo, S., Clark, D.S., Chen, F., Zhang, T., Mackie, R.I., Pennacchio, L.A., Tringe, S.G., Visel, A., Woyke, T., Wang, Z., Rubin, E.M., 2011. Metagenomic Discovery of Biomass-Degrading Genes and Genomes from Cow Rumen. Science 331, 463467.

Holmes, R.K., 2000. Biology and Molecular Epidemiology of Diphtheria Toxin and the tox Gene. J. Infect. Dis. 181, S156-S167. doi:10.1086/315554

Horvat, C.N., 2012. Development and Applications of Nitroreductase Activated Masked Fluorophores.

Hsu, C., Hou, M., Hong, J., Wu, J., Her, G.M., 2010. Inducible Male Infertility by Targeted Cell Ablation in Zebrafish Testis. Mar. Biotechnol. 12, 466-78.

Hult, K., Berglund, P., 2007. Enzyme Promiscuity: Mechanism and Applications. Trends Biotechnol. 25, 231-238.

Hu, S.-Y., Lin, P.-Y., Liao, C.-H., Gong, H.-Y., Lin, G.-H., Kawakami, K., Wu, J.-L., 2010. Nitroreductase-Mediated Gonadal Dysgenesis for Infertility Control of Genetically Modified Zebrafish. Mar. Biotechnol. 12, 569-578.

Iqbal, H.A., Feng, Z., Brady, S.F., 2012. Biocatalysts and their Small Molecule Products from Metagenomic Studies. Curr. Opin. Chem. Biol. 16, 109-116.

Iqbal, T., Byrd-Jacobs, C., 2010. Rapid Degeneration and Regeneration of the Zebrafish Olfactory Epithelium after Triton X-100 Application. Chem. Senses 35, 351-361.

Isles, A.R., Ma, D., Milsom, C., Skynner, M.J., Cui, W., Clark, J., Keverne, E.B., Allen, N.D., 2001. Conditional Ablation of Neurones in Transgenic Mice. J. Neurobiol. 47, 183-193.

Ivanova, A., Signore, M., Caro, N., Greene, N.D.E., Copp, A.J., Martinez-Barbera, J.P., 2005. In vivo Genetic Ablation by Cre-Mediated Expression of Diphtheria Toxin Fragment A. Genesis 43, 129-135.

Jaberipour, M., Vass, S.O., Guise, C.P., Grove, J.I., Knox, R.J., Hu, L., Hyde, E.I., Searle, P.F., 2010. Testing Double Mutants of the Enzyme Nitroreductase for Enhanced Cell Sensitisation to Prodrugs: Effects of Combining Beneficial Single Mutations. Biochem. Pharmacol. 79, 102-111.

Jäckel, C., Kast, P., Hilvert, D., 2008. Protein Design by Directed Evolution. Annu. Rev. Biophys. 37, 153-173.

Jadhav, P.M., Sarangapani, R., Ghule, V.D., Prasanth, H., Pandey, R.K., 2013. QuantumChemical Studies on Thermodynamic Feasibility of 1-methyl-2,4,5Trinitroimidazole Processes. J. Mol. Model. 19, 3027-3033.

Jarrom, D., Jaberipour, M., Guise, C.P., Daff, S., White, S.A., Searle, P.F., Hyde, E.I., 2009. Steady-State and Stopped-Flow Kinetic Studies of Three Escherichia coli NfsB mutants with Enhanced Activity for the Prodrug CB1954. Biochemistry (Mosc.) 48, 7665-7672.

Ju, K.-S., Parales, R.E., 2010. Nitroaromatic Compounds, from Synthesis to Biodegradation. Microbiol. Mol. Biol. Rev. MMBR 74, 250-272.

Keyvani, K., Baur, I., Paulus, W., 1999. Tetracycline-Controlled Expression but not Toxicity of an Attenuated Diphtheria Toxin Mutant. Life Sci. 64, 1719-1724.

Khersonsky, O., Tawfik, D., 2010. Enzyme Promiscuity: A Mechanistic and Evolutionary Perspective. Annu. Rev. Biochem. 79, 471-505.

Knox, R.J., Friedlos, F., Marchbank, T., Roberts, J.J., 1991. Bioactivation of CB1954: Reaction of the Active 4-hydroxylamino Derivative with Thioesters to Form the 
Ultimate DNA-DNA Interstrand Crosslinking Species. Biochem. Pharmacol. 42, 1691-1697.

Knox, R.J., Friedlos, F., Sherwood, R.F., Melton, R.G., Anlezark, G.M., 1992. The Bioactivation of 5-(Aziridin-1-yl)-2,4-Dinitrobenzamide (CB1954)-II: A Comparison of an Escherichia coli Nitroreductase and Walker DT Diaphorase. Biochem. Pharmacol. 44, 2297-2301.

Kobori, T., Sasaki, H., Lee, W.C., Zenno, S., Saigo, K., Murphy, M.E.P., Tanokura, M., 2001. Structure and Site-directed Mutagenesis of a Flavoprotein from Escherichia coli That Reduces Nitrocompounds. J. Biol. Chem. 276, 2816-2823. d

Kurumata, M., Takahashi, M., Sakamotoa, A., Ramos, J.L., Nepovim, A., Vanek, T., Hirata, T., Morikawa, H., 2005. Tolerance to, and Uptake and Degradation of 2,4,6-Trinitrotoluene (TNT) are Enhanced by the Expression of a Bacterial Nitroreductase Gene in Arabidopsis thaliana. Z. Für Naturforschung C J. Biosci. 60, 272-278.

Kwak, S.P., Malberg, J.E., Howland, D.S., Cheng, K.-Y., Su, J., She, Y., Fennell, M., Ghavami, A., 2007. Ablation of Central Nervous System Progenitor Cells in Transgenic Rats using Bacterial Nitroreductase System. J. Neurosci. Res. 85, 1183-1193.

Kwak, Y.H., Lee, D.S., Kim, H.B., 2003. Vibrio harveyi Nitroreductase is also a Chromate Reductase. Appl. Environ. Microbiol. 69, 4390-4395.

Lee, G., Kim, K.Y., Chang, C.-H., Kim, M.G., 2012. Thymic Epithelial Requirement for $\gamma \delta \mathrm{T}$ Cell Development Revealed in the Cell Ablation Transgenic System with TSCOT Promoter. Mol. Cells 34, 481-493.

Lee, M.K., Williams, J., Twieg, R.J., Rao, J., Moerner, W.E., 2013. Enzymatic Activation of Nitro-Aryl Fluorogens in Live Bacterial Cells for Enzymatic TurnoverActivated Localization Microscopy. Chem. Sci. 4, 220.

Lee, P., Morley, G., Huang, Q., Fischer, A., Seiler, S., Horner, J.W., Factor, S., Vaidya, D., Jalife, J., Fishman, G.I., 1998. Conditional Lineage Ablation to Model Human Diseases. Proc. Natl. Acad. Sci. U. S. A. 95, 11371-11376.

Lemmon, M.J., van Zijl, P., Fox, M.E., Mauchline, M.L., Giaccia, A.J., Minton, N.P., Brown, J.M., 1997. Anaerobic Bacteria as a Gene Delivery System that is Controlled by the Tumor Microenvironment. Gene Ther. 4, 791-796.

Lieschke, G.J., Currie, P.D., 2007. Animal Models of Human Disease: Zebrafish Swim into View. Nat. Rev. Genet. 8, 353-367.

Lindmark, D.G., Muller, M., 1976. Antitrichomonad Action, Mutagenicity, and Reduction of Metronidazole and Other Nitroimidazoles. Antimicrob. Agents Chemother. 10, 476-482.

Ling, L.L., Schneider, T., Peoples, A.J., Spoering, A.L., Engels, I., Conlon, B.P., Mueller, A., Schäberle, T.F., Hughes, D.E., Epstein, S., Jones, M., Lazarides, L., Steadman, V.A., Cohen, D.R., Felix, C.R., Fetterman, K.A., Millett, W.P., Nitti, A.G., Zullo, A.M., Chen, C., Lewis, K., 2015. A New Antibiotic Kills Pathogens Without Detectable Resistance. Nature 517, 455-459.

Liochev, S.I., Hausladen, A., Fridovich, I., 1999. Nitroreductase A is Regulated as a Member of the soxRS Regulon of Escherichia coli. Proc. Natl. Acad. Sci. U. S. A. 96, 3537-3539.

Li, Q., Karam, S.M., Gordon, J.I., 1996. Diphtheria Toxin-Mediated Ablation of Parietal Cells in the Stomach of Transgenic Mice. J. Biol. Chem. 271, 3671-3676.

Liu, S.-C., Ahn, G.-O., Kioi, M., Dorie, M.-J., Patterson, A.V., Brown, J.M., 2008. Optimized Clostridium-Directed Enzyme Prodrug Therapy Improves the 
Antitumor Activity of the Novel DNA Cross-Linking Agent PR-104. Cancer Res. 68, 7995-8003.

Li, X., Montgomery, J., Cheng, W., Noh, J.H., Hyde, D.R., Li, L., 2012. Pineal Photoreceptor Cells are Required for Maintaining the Circadian Rhythms of Behavioral Visual Sensitivity in Zebrafish. PloS One 7, e40508.

Li, Z., He, X., Wang, Z., Yang, R., Shi, W., Ma, H., 2015. In vivo Imaging and detection of Nitroreductase in Zebrafish by a New Near-Infrared Fluorescence Off-On Probe. Biosens. Bioelectron. 63, 112-116.

Lomovskaya, O., Warren, M.S., Lee, A., Galazzo, J., Fronko, R., Lee, M., Blais, J., Cho, D., Chamberland, S., Renau, T., Leger, R., Hecker, S., Watkins, W., Hoshino, K., Ishida, H., Lee, V.J., 2001. Identification and Characterization of Inhibitors of Multidrug Resistance Efflux Pumps in Pseudomonas aeruginosa: Novel Agents for Combination Therapy. Antimicrob. Agents Chemother. 45, 105-116.

Low, K.B., Ittensohn, M., Luo, X., Zheng, L.-M., King, I., Pawelek, J.M., Bermudes, D., 2004. Construction of VNP20009: a Novel, Genetically Stable AntibioticSensitive Strain of Tumor-Targeting Salmonella for Parenteral Administration in Humans. Methods Mol. Med. 90, 47-60.

Lukashev, A.N., Fuerer, C., Chen, M.-J., Searle, P., Iggo, R., 2005. Late Expression of Nitroreductase in an Oncolytic Adenovirus Sensitizes Colon Cancer Cells to the Prodrug CB1954. Hum. Gene Ther. 16, 1473-1483.

Macary, G., Rossert, J., Bruneval, P., Mandet, C., Bélair, M.-F., Houillier, P., Duong Van Huyen, J.-P., 2010. Transgenic Mice Expressing Nitroreductase Gene Under the Control of the Podocin Promoter: a New Murine Model of Inductible Glomerular Injury. Virchows Arch. Int. J. Pathol. 456, 325-337.

Mathias, J.R., Zhang, Z., Saxena, M.T., Mumm, J.S., 2014. Enhanced Cell-Specific Ablation in Zebrafish Using a Triple Mutant of Escherichia Coli Nitroreductase. Zebrafish 11, 85-97.

McCormack, E., Silden, E., West, R.M., Pavlin, T., Micklem, D.R., Lorens, J.B., Haug, B.E., Cooper, M.E., Gjertsen, B.T., 2013. Nitroreductase, a Near-Infrared Reporter Platform for In Vivo Time-Domain Optical Imaging of Metastatic Cancer. Cancer Res. 73, 1276-1286.

McNeish, I.A., Green, N.K., Gilligan, M.G., Ford, M.J., Mautner, V., Young, L.S., Kerr, D.J., Searle, P.F., 1998. Virus Directed Enzyme Prodrug Therapy for Ovarian and Pancreatic Cancer using Retrovirally Delivered E. coli Nitroreductase and CB1954. Gene Ther. 5, 1061-1069.

Montgomery, J.E., Parsons, M.J., Hyde, D.R., 2010. A Novel Model of Retinal Ablation Demonstrates that the Extent of Rod Cell Death Regulates the Origin of the Regenerated Zebrafish Rod Photoreceptors. J. Comp. Neurol. 518, 800-814.

Naik, S., Russell, S.J., 2009. Engineering Oncolytic Viruses to Exploit Tumor Specific Defects in Innate Immune Signaling Pathways. Expert Opin. Biol. Ther. 9, 11631176.

Nair, N.U., Zhao, H., 2008. Evolution in Reverse: Engineering a D-Xylose-Specific Xylose Reductase. ChemBioChem 9, 1213-1215.

Nakanishi, M., Yatome, C., Ishida, N., Kitade, Y., 2001. Putative ACP Phosphodiesterase Gene (acpD) Encodes an Azoreductase. J. Biol. Chem. 276, 46394-46399.

Niehaus, F., Gabor, E., Wieland, S., Siegert, P., Maurer, K.H., Eck, J., 2011. Enzymes for the Laundry Industries: Tapping the Vast Metagenomic Pool of Alkaline Proteases. Microb. Biotechnol. 4, 767-776.

Nivinskas, H., Staskeviciene, S., Sarlauskas, J., Koder, R.L., Miller, A.F., Cenas, N., 2002. Two-Electron Reduction of Quinones by Enterobacter cloacae 
NAD(P)H:Nitroreductase: Quantitative Structure-Activity Relationships. Arch. Biochem. Biophys. 403, 249-258.

Ofori-Adjei, D., Dodoo, A.N.O., Appiah-Danquah, A., Couper, M., 2008. A Review of the Safety of Niclosamide, Pyrantel, Triclabendazole and Oxamniquine. Int. J. Risk Saf. Med. 20, 113-122.

Olekhnovich, I.N., Goodwin, A., Hoffman, P.S., 2009. Characterization of the NAD(P)H Oxidase and Metronidazole Reductase Activities of the RdxA Nitroreductase of Helicobacter pylori. FEBS J. 276, 3354-3364.

Owen, J.G., Robins, K.J., Parachin, N.S., Ackerley, D.F., 2012. A Functional Screen for Recovery of 4'-Phosphopantetheinyl Transferase and Associated Natural Product Biosynthesis Genes from Metagenome Libraries. Environ. Microbiol. 14, 1198 1209.

Palmiter, R.D., Behringer, R.R., Quaife, C.J., Maxwell, F., Maxwell, I.H., Brinster, R.L., 1987. Cell Lineage Ablation in Transgenic Mice by Cell-Specific Expression of a Toxin gene. Cell 50, 435-443.

Parachin, N.S., Gorwa-Grauslund, M.F., 2011. Isolation of xylose Isomerases by Sequence-and Function-Based Screening from a Soil Metagenomic Library. Biotechnol. Biofuels 4, 9.

Park, C.-H., Gonzalez, C., Ackerley, D., Keyhan, M., Matin, A., 2001. Molecular Engineering of Soluble Bacterial Proteins with Chromate Reductase Activity. Proc. 1st Int. Conf. Remediat. Con- Taminated Sediments Venice Italy Vol III Hinchee RE Porta Pellei M Eds Columb. OH Batelle Press Pp 103-111 103-112.

Parry, R., Nishino, S., Spain, J., 2010. Naturally-Occurring Nitro Compounds. Nat. Prod. Rep. 28, 152-167.

Patel, P., Young, J.G., Mautner, V., Ashdown, D., Bonney, S., Pineda, R.G., Collins, S.I., Searle, P.F., Hull, D., Peers, E., Chester, J., Wallace, D.M., Doherty, A., Leung, H., Young, L.S., James, N.D., 2009. A Phase I/II Clinical Trial in Localized Prostate Cancer of an Adenovirus Expressing Nitroreductase with CB1984. Mol. Ther. 17, 1292-1299.

Patterson, A.V., Saunders, M.P., Greco, O., 2003. Prodrugs in Genetic Chemoradiotherapy. Curr. Pharm. Des. 9, 2131-54.

Paulus, W., Baur, I., Oberer, D.M., Breakefield, X.O., Reeves, S.A., 1997. Regulated Expression of the Diphtheria Toxin A gene in Human Glioma Cells using Prokaryotic Transcriptional Control Elements. J. Neurosurg. 87, 89-95.

Peterson, F.J., Mason, R.P., Hovsepian, J., Holtzman, J.L., 1979. Oxygen-sensitive andinsensitive Nitroreduction by Escherichia coli and Rat Hepatic Microsomes. J. Biol. Chem. 254, 4009-4014.

Piel, J., 2011. Approaches to Capturing and Designing Biologically Active Small Molecules Produced by Uncultured Microbes. Annu. Rev. Microbiol. 65, 431453.

Pisharath, H., 2007. Validation of Nitroreductase, a Prodrug-activating Enzyme, Mediated Cell Death in Embryonic Zebrafish (Danio rerio). Comp. Med. 57, 241246.

Pisharath, H., Rhee, J.M., Swanson, M.A., Leach, S.D., Parsons, M.J., 2007. Targeted Ablation of Beta Cells in the Embryonic Zebrafish Pancreas using E. coli Nitroreductase. Mech. Dev. 124, 218-229.

Pitsawong, W., Hoben, J.P., Miller, A.-F., 2014. Understanding the Broad Substrate Repertoire of Nitroreductase Based on its Kinetic Mechanism. J. Biol. Chem. jbc.M113.547117. 
Prosser, G.A., Copp, J.N., Mowday, A.M., Guise, C.P., Syddall, S.P., Williams, E.M., Horvat, C.N., Swe, P.M., Ashoorzadeh, A., Denny, W.A., Smaill, J.B., Patterson, A.V., Ackerley, D.F., 2013. Creation and Screening of a Multi-Family Bacterial Oxidoreductase Library to Discover Novel Nitroreductases that Efficiently Activate the Bioreductive Prodrugs CB1954 and PR-104A. Biochem. Pharmacol. $85,1091-1103$.

Prosser, G.A., Copp, J.N., Syddall, S.P., Williams, E.M., Smaill, J.B., Wilson, W.R., Patterson, A.V., Ackerley, D.F., 2010. Discovery and Evaluation of Escherichia coli Nitroreductases that Activate the Anti-Cancer Prodrug CB1954. Biochem. Pharmacol. 79, 678-687.

Race, P.R., Lovering, A.L., White, S.A., Grove, J.I., Searle, P.F., Wrighton, C.W., Hyde, E., 2007. Kinetic and Structural Characterisation of Escherichia coli Nitroreductase Mutants Showing Improved Efficacy for the Prodrug Substrate CB1954. J. Mol. Biol. 368, 481-492.

Rafii, F., Cerniglia, C.E., 1995. Reduction of Azo Dyes and Nitroaromatic Compounds by Bacterial Enzymes from the Human Intestinal Tract. Environ. Health Perspect. 103, 17-19.

Rafil, F., Franklin, W., Heflich, R.H., Cerniglia, C.E., 1991. Reduction of Nitroaromatic Compounds by Anaerobic Bacteria Isolated from the Human Gastrointestinal Tract. Appl. Environ. Microbiol. 57, 962-968.

Rau, J., Stolz, A., 2003. Oxygen-Insensitive Nitroreductases NfsA and NfsB of Escherichia coli Function Under Anaerobic Conditions as Lawsone-Dependent Azo Reductases. Appl. Environ. Microbiol. 69, 3448-3455.

Reetz, M.T., Brunner, B., Schneider, T., Schulz, F., Clouthier, C.M., Kayser, M.M., 2004. Directed Evolution as a Method To Create Enantioselective Cyclohexanone Monooxygenases for Catalysis in Baeyer-Villiger Reactions. Angew. Chem. Int. Ed. 43, 4075-4078.

Robins, K.J., Hooks, D.O., Rehm, B.H.A., Ackerley, D.F., 2013. Escherichia coli NemA Is an Efficient Chromate Reductase That Can Be Biologically Immobilized to Provide a Cell Free System for Remediation of Hexavalent Chromium. PLoS ONE 8, e59200. doi:10.1371/journal.pone.0059200

Roeser, T., Baier, H., 2003. Visuomotor Behaviors in Larval Zebrafish after GFP-Guided Laser Ablation of the Optic Tectum. J. Neurosci. 23, 3726-3734.

Roldán, M.D., Pérez-Reinado, E., Castillo, F., Moreno-Vivián, C., 2008. Reduction of Polynitroaromatic Compounds: the Bacterial Nitroreductases. FEMS Microbiol. Rev. 32, 474-500.

Sheard, C.E., Double, J.A., Berenbaum, M.C., 1971. The Sensitivity to Chemotherapeutic Agents of a Rat Tumour Grown in Immunosuppressed Mice. Br. J. Cancer 25, 838-844.

Shi, Y., Zhang, S., Zhang, X., 2013. A Novel Near-Infrared Fluorescent Probe for Selectively Sensing NItroreductase (NTR) in an Aqueous Medium. Analyst 138, 1952-1955.

Simon, C., Herath, J., Rockstroh, S., Daniel, R., 2009. Rapid Identification of Genes Encoding DNA Polymerases by Function-Based Screening of Metagenomic Libraries Derived from Glacial Ice. Appl. Environ. Microbiol. 75, 2964-2968.

Slanchev, K., Stebler, J., Cueva-Méndez, G. de la, Raz, E., 2005. Development without Germ Cells: The role of the Germ Line in Zebrafish Sex Differentiation. Proc. Natl. Acad. Sci. U. S. A. 102, 4074-4079.

Smets, B.F., Yin, H., Esteve-Nuñez, A., 2007. TNT Biotransformation: when Chemistry Confronts Mineralization. Appl. Microbiol. Biotechnol. 76, 267-277. 
Soria, B., Roche, E., Berná, G., León-Quinto, T., Reig, J.A., Martín, F., 2000. InsulinSecreting Cells Derived from Embryonic Stem Cells Normalize Glycemia in Streptozotocin-Induced Diabetic Mice. Diabetes 49, 157-162.

Soustelle, L., Aigouy, B., Asensio, M.-L., Giangrande, A., 2008. UV Laser Mediated Cell Selective Destruction by Confocal Microscopy. Neural Develop. 3, 11.

Stewart, E.J., 2012. Growing Unculturable Bacteria. J. Bacteriol. 194, 4151-4160.

Su, J., Guise, C., Wilson, W., 2013. FSL-61 is a 6-Nitroquinolone Fluorogenic Probe for One-Electron Reductases in Hypoxic Cells. Biochem. J.

Swe, P.M., Copp, J.N., Green, L.K., Guise, C.P., Mowday, A.M., Smaill, J.B., Patterson, A.V., Ackerley, D.F., 2012. Targeted Mutagenesis of the Vibrio fischeri Flavin Reductase FRase I to Improve Activation of the Anticancer Prodrug CB1954. Biochem. Pharmacol. 84, 775-783.

Takeda, K., Sato, J., Goto, K., Fujita, T., Watanabe, T., Abo, M., Yoshimura, E., Nakagawa, J., Abe, A., Kawasaki, S., Niimura, Y., 2010. Escherichia coli Ferredoxin-NADP+ Reductase and Oxygen-Insensitive Nitroreductase are Capable of Functioning as Ferric Reductase and of Driving the Fenton Reaction. BioMetals 23, 727-737.

Thomas, P., Smart, T.G., 2005. HEK293 cell line: A Vehicle for the Expression of Recombinant Proteins. J. Pharmacol. Toxicol. Methods, Electrophysiological Methods in Neuropharmacology 51.

Thorne, S.H., Barak, Y., Liang, W., Bachmann, M.H., Rao, J., Contag, C.H., Matin, A., 2009. CNOB/ChrR6, a New Prodrug Enzyme Cancer Chemotherapy. Mol. Cancer Ther. 8, 333-341.

Torsvik, V., Goks?yr, J., Daae, F.L., 1990. High Diversity in DNA of Soil Bacteria. Appl. Environ. Microbiol. 56, 782-787.

Torsvik, V., Øvreås, L., Thingstad, T.F., 2002. Prokaryotic Diversity-Magnitude, Dynamics, and Controlling Factors. Science 296, 1064-1066.

Tracewell, C.A., Arnold, F.H., 2009. Directed Enzyme Evolution: Climbing Fitness Peaks One Amino Acid at a Time. Curr. Opin. Chem. Biol. 13, 3-9.

Van Dillewijn, P., Couselo, J.L., Corredoira, E., Delgado, A., Wittich, R.-M., Ballester, A., Ramos, J.L., 2008. Bioremediation of 2,4,6-Trinitrotoluene by Bacterial Nitroreductase Expressing Transgenic Aspen. Environ. Sci. Technol. 42, 74057410.

Varadarajan, N., Georgiou, G., Iverson, B.L., 2008a. An Engineered Protease that Cleaves Specifically after Sulfated Tyrosine. Angew. Chem. Int. Ed Engl. 47, 7861-7863.

Varadarajan, N., Rodriguez, S., Hwang, B.-Y., Georgiou, G., Iverson, B.L., 2008b. Highly Active and Selective Endopeptidases with Programmed Substrate Specificities. Nat. Chem. Biol. 4, 290-294.

Vass, S.O., Jarrom, D., Wilson, W.R., Hyde, E.I., Searle, P.F., 2009. E. coli NfsA: an Alternative Nitroreductase for Prodrug Activation Gene Therapy in Combination with CB1954. Br. J. Cancer 100, 1903-1911.

Wang, K., Li, G., Yu, S.Q., Zhang, C.T., Liu, Y.H., 2010. A Novel Metagenome-Derived $\beta$-galactosidase: Gene Cloning, Overexpression, Purification and Characterization. Appl. Microbiol. Biotechnol. 88, 155-165.

Warnecke, F., Luginbühl, P., Ivanova, N., Ghassemian, M., Richardson, T.H., Stege, J.T., Cayouette, M., McHardy, A.C., Djordjevic, G., Aboushadi, N., Sorek, R., Tringe, S.G., Podar, M., Martin, H.G., Kunin, V., Dalevi, D., Madejska, J., Kirton, E., Platt, D., Szeto, E., Salamov, A., Barry, K., Mikhailova, N., Kyrpides, N.C., Matson, E.G., Ottesen, E.A., Zhang, X., Hernández, M., Murillo, C., Acosta, L.G., 
Rigoutsos, I., Tamayo, G., Green, B.D., Chang, C., Rubin, E.M., Mathur, E.J., Robertson, D.E., Hugenholtz, P., Leadbetter, J.R., 2007. Metagenomic and Functional Analysis of Hindgut Microbiota of a Wood-Feeding Higher Termite. Nature 450, 560-565.

Weedon, S.J., Green, N.K., McNeish, I.A., Gilligan, M.G., Mautner, V., Wrighton, C.J., Mountain, A., Young, L.S., Kerr, D.J., Searle, P.F., 2000. Sensitisation of Human Carcinoma Cells to the Prodrug CB1954 by Adenovirus Vector-Mediated Expression of E. coli Nitroreductase. Int. J. Cancer 86, 848-854.

White, D.T., Mumm, J.S., 2013. The Nitroreductase System of Inducible Targeted Ablation Facilitates Cell-Specific Regenerative Studies in Zebrafish. Methods 62, 232-240.

Williams, E.M., 2013. Development of Bacterial Nitroreductase Enzymes for Noninvasive Imaging in Cancer Gene Therapy.

Wilson, W.R., Hay, M.P., 2011. Targeting Hypoxia in Cancer Therapy. Nat. Rev. Cancer 11, 393-410. doi:10.1038/nrc3064

Xu, B., Xu, W., Yang, F., Li, J., Yang, Y., Tang, X., Mu, Y., Zhou, J., Huang, Z., 2013. Metagenomic Analysis of the Pygmy Loris Fecal Microbiome Reveals Unique Functional Capacity Related to Metabolism of Aromatic Compounds. PLoS ONE 8, e56565.

Yamaizumi, M., Mekada, E., Uchida, T., Okada, Y., 1978. One Molecule of Diphtheria Toxin fragment A Introduced into a Cell Can Kill the Cell. Cell 15, 245-250.

Yamazoe, S., McQuade, L.E., Chen, J.K., 2014. Nitroreductase-Activatable Morpholino Oligonucleotides for In vivo Gene Silencing. ACS Chem. Biol. 9, 1985-1990.

Yang, C.-T., Johnson, S.L., 2006. Small molecule-Induced Ablation and Subsequent Regeneration of Larval Zebrafish Melanocytes. Development 133, 3563-3573.

Yano, T., Oue, S., Kagamiyama, H., 1998. Directed Evolution of an Aspartate Aminotransferase with New Substrate Specificities. Proc. Natl. Acad. Sci. U. S. A. 95, 5511-5515.

Zenno, S., Kobori, T., Tanokura, M., Saigo, K., 1998. Conversion of NfsA, the Major Escherichia coli Nitroreductase, to a Flavin Reductase with an Activity Similar to That of Frp, a Flavin Reductase in Vibrio harveyi, by a Single Amino Acid Substitution. J. Bacteriol. 180, 422-425.

Zenno, S., Koike, H., Tanokura, M., Saigo, K., 1996. Conversion of NfsB, a Minor Escherichia coli Nitroreductase, to a Flavin Reductase Similar in Biochemical Properties to FRase I, the Major Flavin Reductase in Vibrio fischeri, by a Single Amino Acid Substitution. J. Bacteriol. 178, 4731-4733.

Zenno, S., Saigo, K., Kanoh, H., Inouye, S., 1994. Identification of the Gene Encoding the Major $\mathrm{NAD}(\mathrm{P}) \mathrm{H}-$ flavin Oxidoreductase of the Bioluminescent Bacterium Vibrio fischeri ATCC 7744. J. Bacteriol. 176, 3536-3543.

Zhao, X.-F., Ellingsen, S., Fjose, A., 2009. Labelling and Targeted Ablation of Specific Bipolar Cell Types in the Zebrafish Retina. BMC Neurosci. 10, 107.

Zhitkovich, A., 2011. Chromium in Drinking Water: Sources, Metabolism, and Cancer Risks. Chem. Res. Toxicol. 24, 1617-1629. doi:10.1021/tx200251t

Zhou, W., Hildebrandt, F., 2012. Inducible Podocyte Injury and Proteinuria in Transgenic Zebrafish. J. Am. Soc. Nephrol. 23, 1039-1047. 


\section{Appendix}
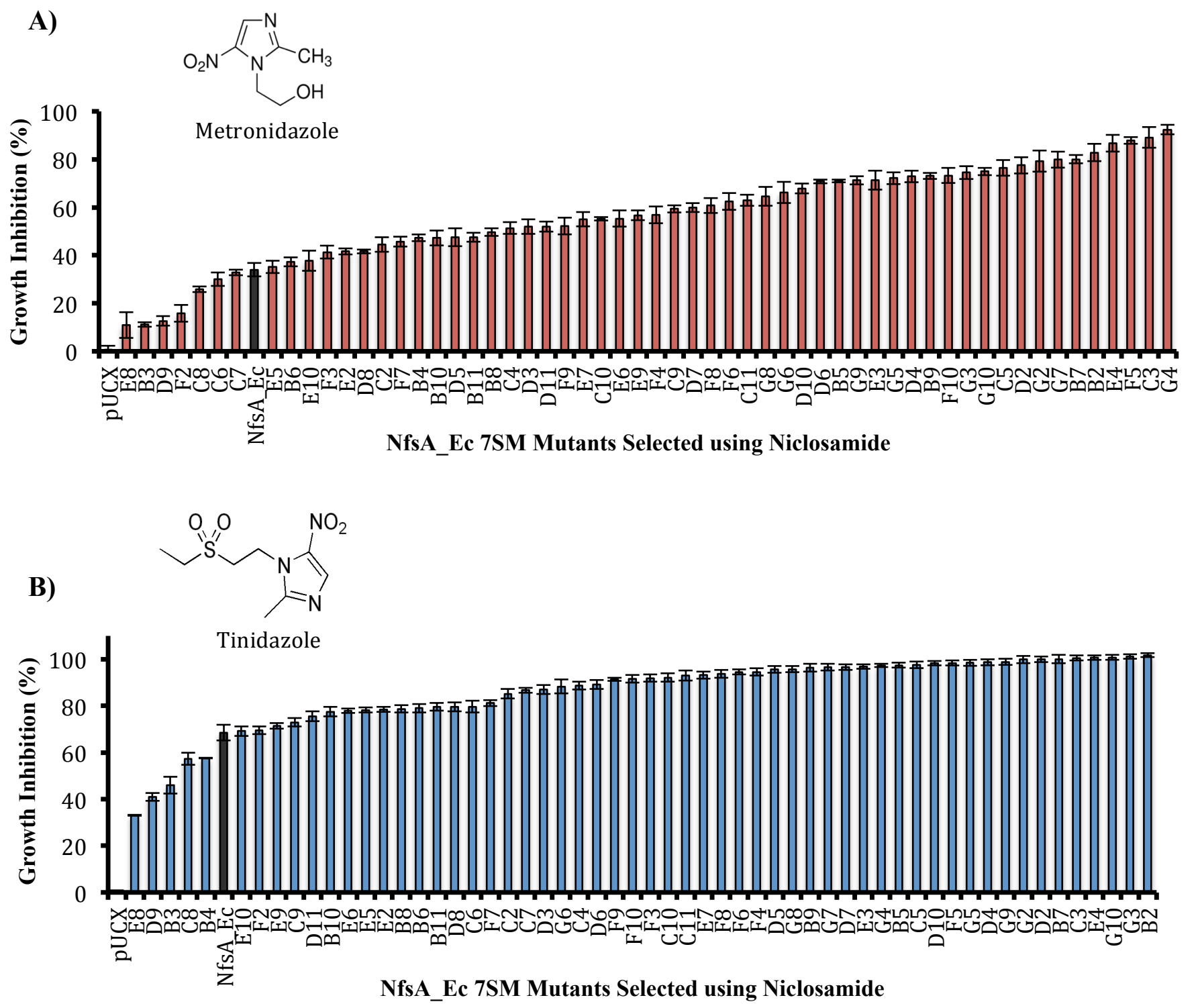

Appendix Figure 8.1 - Metronidazole and tinidazole induced growth inhibition response in $E$. coli SOS-R4 overexpressing NfsA_Ec 7SM mutants preselected on niclosamide. A total of 57 NfsA_Ec 7SM mutant colonies were randomly selected from LB agar supplemented with $0.5 \mu \mathrm{M}$ niclosamide and challenged with either metronidazole or tinidazole in replicate growth inhibition assays. Mid-exponential cultures from each of the 57 colonies were challenged in 384 well plates with either A) $50 \mu \mathrm{M}$ metronidazole or B) $50 \mu \mathrm{M}$ tinidazole for 3 hours. Growth was measured as the difference in culture turbidity $\left(\mathrm{OD}_{600}\right)$ between pre $(\mathrm{t}=0 \mathrm{~h})$ and post $(\mathrm{t}=3 \mathrm{~h})$ drug challenged cultures. A DMSO control (unchallenged) was also included for each of the 57 7SM mutants. For each strain, growth inhibition was measured as the percentage decrease in growth between each challenged culture and its respective unchallenged control. Data are representative of three independent experiments \pm SEM. 


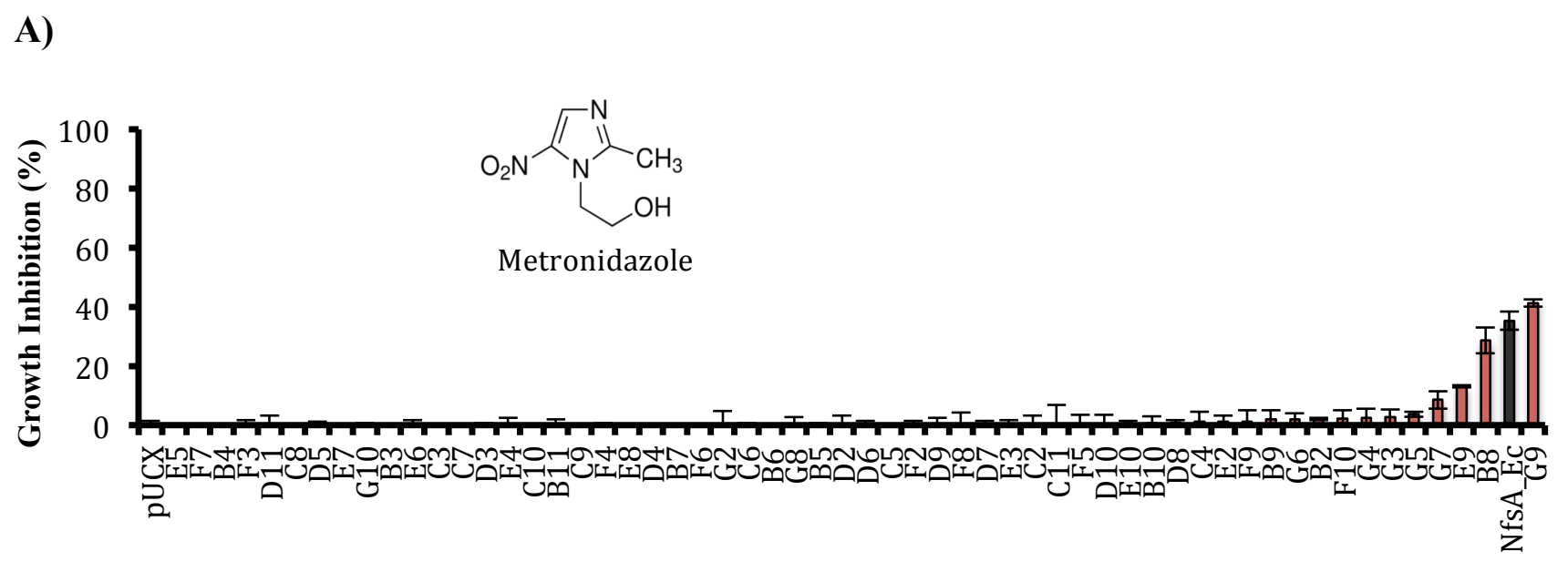

NfsA_Ec 7SM Mutants Lacking Niclosamide Selection

B)

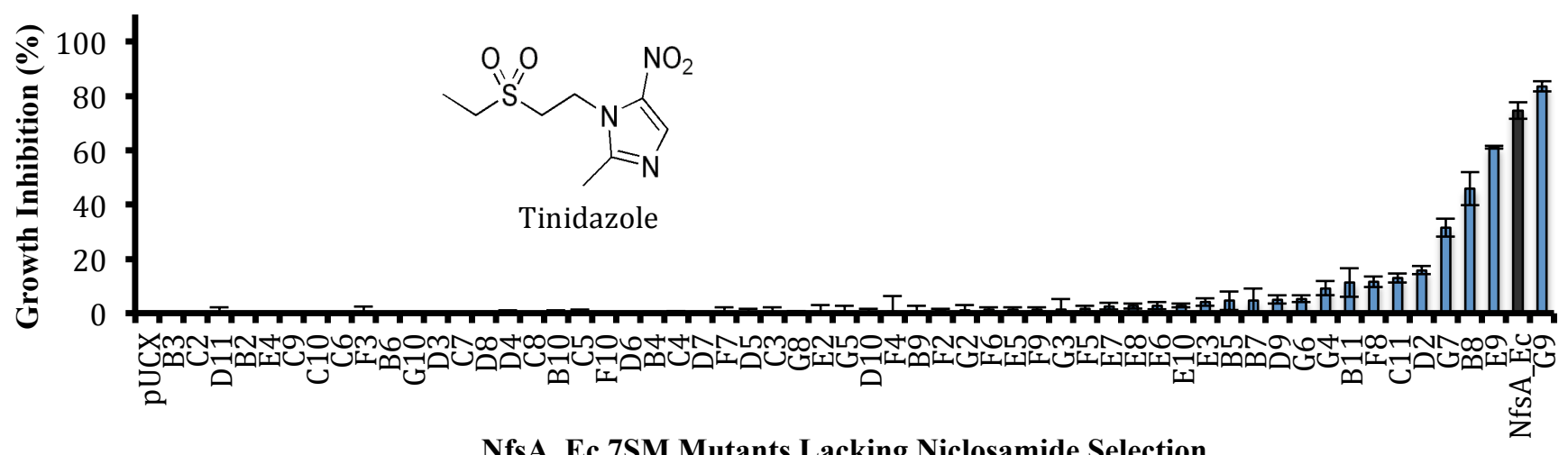

NfsA_Ec 7SM Mutants Lacking Niclosamide Selection

Appendix Figure 8.2 - Metronidazole and tinidazole induced growth inhibition response in $E$. coli SOS-R4 overexpressing NfsA_Ec 7SM mutants lacking niclosamide selection. A total of 57 NfsA_Ec 7SM mutant colonies were randomly selected from LB agar supplemented with $0.5 \mu \mathrm{M}$ niclosamide and challenged with either metronidazole or tinidazole in replicate growth inhibition assays. Mid-exponential cultures from each of the 57 colonies were challenged in 384 well plates with either A) $50 \mu \mathrm{M}$ metronidazole or B) $50 \mu \mathrm{M}$ tinidazole for 3 hours. Growth was measured as the difference in culture turbidity $\left(\mathrm{OD}_{600}\right)$ between pre $(\mathrm{t}=0 \mathrm{~h})$ and post $(\mathrm{t}=3 \mathrm{~h})$ drug challenged cultures. A DMSO control (unchallenged) was also included for each of the 57 7SM mutants. For each strain, growth inhibition was measured as the percentage decrease in growth between each challenged culture and its respective unchallenged control. Data are representative of three independent experiments \pm SEM. 


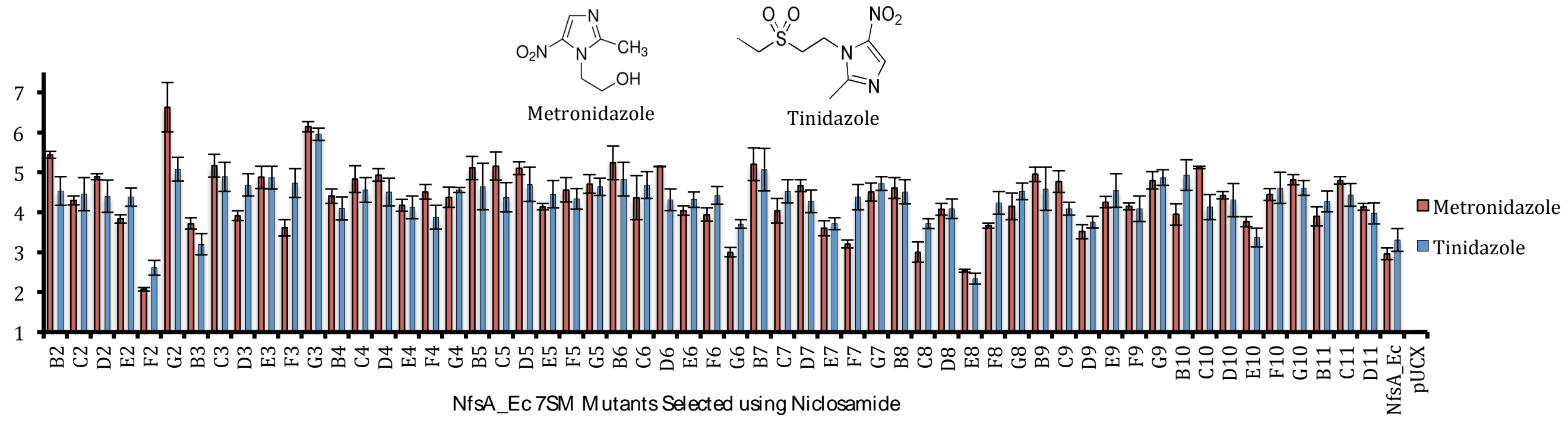

E. coli

Mid-exponential cultures of $57 \mathrm{NfsA}$ Ec $7 \mathrm{SM}$ mutants selected on agar containing $0.5 \mu \mathrm{M}$ niclosamide were challenged in 384 well plates containing either $5 \mu \mathrm{M}$ metronidazole or $5 \mu \mathrm{M}$ tinidazole for 3 hours, after which GFP fluorescence $(488 \mathrm{ex} / 510 \mathrm{em})$ and culture turbidity $\left(\mathrm{OD}_{600}\right)$ were recorded. A DMSO control (unchallenged) was also included for each of the 53 colony cultures. The raw GFP values of each challenged culture and their respective unchallenged control were normalized by $\mathrm{OD}_{600}$. GFP fold induction was then calculated from the difference between the normalized GFP values of each challenged culture and its respective unchallenged control. SOS-R4 clones expressing NfsB_Vv, NfsA_Pp, and NfsA_Ec are included as controls. pUCX represents SOS-R4 cells containing an empty pUCX plasmid. Data are representative of three independent experiments \pm SEM using cultures of the same 7SM NfsA_Ec mutants. 

